Datenrecht und neue Technologien

Lisa Käde

\title{
Kreative Maschinen \\ und Urheberrecht
}

Die Machine Learning-Werkschöpfungskette vom Training über Modellschutz bis zu Computational Creativity 
Datenrecht und neue Technologien

Herausgegeben von

Prof. Dr. Matthias Cornils

Prof. Dr. Martin Ebers

Prof. Dr. Mario Martini

Prof. Dr. Dr. Frauke Rostalski

Prof. Dr. Giesela Rühl

Prof. Dr. Björn Steinrötter

Band 2 
Lisa Käde

Kreative Maschinen

und Urheberrecht

Die Machine Learning-Werkschöpfungskette vom Training über Modellschutz bis zu Computational Creativity 
The book processing charge was funded by the Baden-Württemberg Ministry of Science, Research and Arts in the funding programme Open Access Publishing and the University of Freiburg.

Dissertation an der rechtswissenschaftlichen Fakultät

der Albert-Ludwigs-Universität Freiburg

Dekan: Prof. Dr. Jan von Hein

Mündliche Prüfung 10./11.05.2021 in Freiburg

Erstgutachter: Prof. Dr. Thomas Dreier, M.C. J.

Zweitgutachter: Prof. Dr. Maximilian Haedicke

Die Deutsche Nationalbibliothek verzeichnet diese Publikation in der Deutschen Nationalbibliografie; detaillierte bibliografische Daten sind im Internet über http://dnb.d-nb.de abrufbar.

Zugl.: Freiburg, Univ., Diss., 2021

\section{Auflage 2021}

() Lisa Käde

Publiziert von

Nomos Verlagsgesellschaft mbH \& Co. KG

Waldseestraße 3-5 | 76530 Baden-Baden

www.nomos.de

Gesamtherstellung:

Nomos Verlagsgesellschaft mbH \& Co. KG

Waldseestraße 3-5 | 76530 Baden-Baden

ISBN (Print): 978-3-8487-7231-5

ISBN (ePDF): 978-3-7489-1245-3

DOI: https://doi.org/10.5771/9783748912453

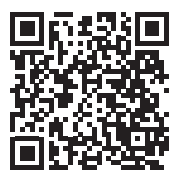

Onlineversion

Nomos elibrary

\section{(c) $(\odot \odot$}

Dieses Werk ist lizenziert unter einer Creative Commons Namensnennung Weitergabe unter gleichen Bedingungen 4.0 International Lizenz. 
Meinen Eltern 


\section{Vorwort}

Die vorliegende Arbeit wurde im Wintersemester 2020/2021 von der rechtswissenschaftlichen Fakultät der Albert-Ludwigs-Universität Freiburg als Dissertation angenommen. Sie entstand während meiner Zeit als akademische Mitarbeiterin am Zentrum für Angewandte Rechtswissenschaft (ZAR) am Karlsruher Institut für Technologie. Rechtsprechung und Literatur sind bis Juni 2021 berücksichtigt.

Mein besonderer Dank gilt Herrn Prof. Dr. Thomas Dreier für die hervorragende Betreuung und Unterstützung. Er hat mich stets mit Anregungen und Informationen zu neuen Ideen inspiriert, und mich dazu motiviert, diese Arbeit deutlich interdisziplinärer zu gestalten als vielleicht für juristische Dissertationen üblich. Herrn Prof. Dr. Maximilian Haedicke danke ich für die zügige Erstellung des Zweitgutachtens.

Ganz herzlich danken möchte ich auch meinem Korrekturleseteam Franziska Delleske und Julia Tabori für ihre Bereitschaft, sich auf die Arbeit einzulassen und ihre hilfreichen Anmerkungen, sowie Mark Keinhörster für den „Technik-Check“. Stephanie von Maltzan danke ich für das immer offene Ohr für jegliche Ideen und Gedankenexperimente und die produktiven Diskussionen über verschiedenste Fragestellungen.

Meinen Kolleginnen und Kollegen am ZAR danke ich für die vielen spannenden Unterhaltungen und die tolle gemeinsame Zeit.

Den Herausgebern und dem Verlag Nomos danke ich für die Aufnahme dieser Arbeit in die Schriftenreihe „Datenrecht und Neue Technologien“.

Von Herzen danken möchte ich meiner Familie, allen voran meinen Eltern Karin und Andreas Käde: Danke für Eure unendliche Geduld, Motivation und Inspiration nicht nur bei der Durchsicht und während der Entstehung dieser Dissertation, sondern während meines gesamten Ausbildungsweges und darüber hinaus. Euch ist diese Arbeit gewidmet. 
Inhaltsübersicht

Erster Teil: Einführung 25

$\begin{array}{lll}\S 1 \text { Einleitung } & 27\end{array}$

$\S 2$ Grundlagen 33

Zweiter Teil: Die Maschine lernt - auch anhand urheberrechtlich geschützter Daten?

$\S 3$ Urheberrechtlich relevante Vorgänge im maschinellen Lernprozess

§ 4 Mögliche Ausnahmetatbestände 81

$\S 5$ Ergebnis des zweiten Teils 93

Dritter Teil: Schutzmöglichkeiten für Modelle maschinellen Lernens im Urheberrecht

$\S 6$ Technische Bestandsaufnahme

§ 7 Schutzmöglichkeiten im Urheberrecht und den verwandten Schutzrechten

$\S 8$ Ergebnis des dritten Teils

Vierter Teil: Schutz von KI-Erzeugnissen

§ 9 Schutzvoraussetzungen im Urheberrecht im Kontext von KIErzeugnissen

$\S 10$ Wem gebührt ein möglicher Schutz? 
Fünfter Teil: Computational Creativity - Kreative Künstliche Intelligenz?

§ 12 Intelligenz, Kreativität und ihre Schnittstellen zueinander und zum Recht

$\S 13$ Computational Creativity

$\S 14$ Ergebnis des fünften Teils

Sechster Teil: Gesamtergebnis und Ausblick

$\S 15$ Gesamtergebnis

$\S 16$ Ausblick 269

Mail-Austausch mit Mike Cook, Projekt ANGELINA 283 
Inhaltsverzeichnis

Erster Teil: Einführung 25

$\S 1$ Einleitung 27

A. Problemstellungen 28

B. Gang der Arbeit 29

C. Grenzen der Arbeit 30

$\S 2$ Grundlagen 33

A. Künstliche Intelligenz 33

I. Begriff der künstlichen Intelligenz 33

1. Ursprung 34

2. KI heute 37

a) Definitionsansätze 38

aa) „Klassische“ KI-Definitionen 38

bb) Alternative Definition 40

cc) Hier: Strukturelle Definition 40

b) Funktionsmäßige Kategorisierung 41

II. Zusammenfassung 42

B. Maschinelles Lernen $\quad 42$

I. Definitionen 43

1. Algorithmus 43

2. Lernen 43

3. Machine Learning 44

4. Modell 44

II. Untersuchte Technologien 45

1. Entscheidungsbäume bzw. Random Forest-Modelle 46

2. Künstliche neuronale Netze 47

3. Sonderfall: Generative Adversarial Networks 50

III. Einige grundlegende Begriffe $\quad 52$

1. Klassifikation, Klassifizierung 52

2. Regression 53

3. Überwachtes Lernen (Supervised Learning) 53

4. Unüberwachtes Lernen (Unsupervised Learning) 54 
5. Lernen durch Verstärkung (Reinforcement Learning)

6. Parameter, Gewichte, Biases, Hyperparameter, Topologie, Schichten, Netzstruktur, Architektur

7. Trainingsprozess, Trainingsdaten, Trainingsergebnisse 55

IV. Technische Einordnung 57

1. Verhältnis des Machine Learning zu Text und Data Mining 58

2. Verhältnis des Machine Learning zu Expertensystemen $\quad 59$

V. Zusammenfassung

Zweiter Teil: Die Maschine lernt - auch anhand urheberrechtlich geschützter Daten?

$\S 3$ Urheberrechtlich relevante Vorgänge im maschinellen Lernprozess

A. Technische Beleuchtung des maschinellen Lernprozesses

I. Einlesevorgang und Analyse bzw. Aufbereitung der

Trainingsdaten

1. Einlesen einzelner oder aller Datensätze

2. Umwandlung der Daten in ein maschinenlesbares Format 65

II. Trainingsvorgang

B. Urheberrechtliche Relevanz des Einlese- und des Trainingsvorgangs 68

I. Vervielfältigung, § 16 UrhG

II. Bearbeitung oder Umgestaltung, § $23 \mathrm{~S}$. 1 UrhG

1. Dem Original dienende Funktion, Anpassung an andere Nutzungsformen

2. Freie Benutzung?

3. Einwilligungserfordernis bei Veröffentlichung oder Verwertung

a) Bearbeitung der Trainingsdatenbank?

b) Veröffentlichung oder Verwertung der Bearbeitung 73

aa) Veröffentlichung

bb) Verwertung

c) Zwischenergebnis

III. Zusammenhang zwischen Trainingsdaten und Output generativer Modelle

IV. Exkurs: Manipulierte ML-Modelle

1. Beispiel: Least Significant Bit Attack 
2. Urheberrechtliche Bewertung 78

$\begin{array}{ll}\text { V. Zusammenfassung } & 78\end{array}$

$\S 4$ Mögliche Ausnahmetatbestände $\quad 81$

A. Situation de lege lata $\quad 81$

I. Zulässigkeit sowohl der Vervielfältigung als auch der Bearbeitung gem. § 60d UrhG? 81

II. Zulässigkeit der Vervielfältigung gem. § 44a UrhG? 83

1. Flüchtige Vervielfältigungen $\quad 84$

2. Rechtmäßige Nutzung 84

3. Vorübergehende Vervielfältigung rechtswidriger Quellen 84

4. Zwischenergebnis 85

B. Situation nach zu erwartenden Änderungen durch die Umsetzung der DSM-Richtlinie $\quad 86$

I. Was ist umzusetzen? 86

II. Wie erfolgt die Umsetzung? 87

1. Welche Auswirkungen ergeben sich für den ML-Prozess? 90

C. Zusammenfassung 91

$\S 5$ Ergebnis des zweiten Teils 93

Dritter Teil: Schutzmöglichkeiten für Modelle maschinellen Lernens im Urheberrecht 95

§ 6 Technische Bestandsaufnahme 99

A. Grundlegende Begriffe 99

I. Frameworks und Bibliotheken 100

II. API 100

III. Objekte und Funktionen 100

B. Grundbausteine für ML-Modelle: Frameworks, Bibliotheken, APIs 103

$\begin{array}{ll}\text { C. Quellcode } & 104\end{array}$

D. Trainiertes Modell in Python 104

I. Vorab: Einsatz eines trainierten Modells 105

II. Begriff des trainierten Modells 107

1. „Trainiertes Modell“ in TensorFlow 107

2. „Trainiertes Modell“ in Keras 109

3. „Trainiertes Modell“ in PyTorch 110

4. „Trainiertes Modell“ in Scikit-Learn 111 
III. Zusammenfassung und Definition 112

E. Trainierter Random Forest in $R \quad 113$

F. Trainierte Parameter 114

G. Hyperparameter 115

H. Zusammenfassung 116

$\S 7$ Schutzmöglichkeiten im Urheberrecht und den verwandten Schutzrechten

A. Einleitung

I. Forschungsstand

II. Hier gewählter Lösungsansatz

B. Trainiertes ML-Modell in Python (insb. trainiertes KNN)

I. Schutz als Datenbankwerk gem. § 4 Abs. 2 UrhG 118

1. Datenbankwerk

a) Sammlung

aa) TensorFlow

bb) Keras

cc) PyTorch

dd) Scikit-Learn

ee) Zusammenfassung und Subsumtion

b) Unabhängige Elemente

c) Systematische oder methodische Anordnung

d) Zugänglichkeit der Elemente

e) Zwischenergebnis

2. Persönliche geistige Schöpfung

a) Persönliche oder eigene geistige Schöpfung?

b) Schöpfung in Auswahl oder Anordnung

aa) Auswahl

bb) Anordnung

cc) Anordnung für Datenbankwerke i. d. R. programmseitig vorgegeben

dd) Schöpfungsspielraum in der Auswahl

3. Ergebnis

4. Wer ist der Urheber?, oder: Schutzumfang und Folgen 131

II. Investitionsschutz gem. $§ \S 87$ a ff. UrhG

1. Datenbank

2. Investitionsgegenstand

3. Wesentlichkeit der Investition

4. Ergebnis 
5. Schutzumfang und Folgen 135

III. Schutz als Computerprogramm gem. §69 a UrhG 135

1. Trainiertes Modell als Computerprogramm 136

a) Begriffsklärung Computerprogramm 136

b) (Keine) Einordnung von ML-Modellen als

Computerprogramm in der Literatur

c) Berücksichtigung der Bestandteile eines trainierten Modells für den Computerprogrammbegriff 138

aa) Quellcode 140

bb) Hyperparameter und Parameter 141

cc) Kombination zur Laufzeit 142

dd) Sonstige Schutzgegenstände 143

ee) Zusammenfassung: infrage kommende Schutzgegenstände

2. Eigene geistige Schöpfung 144

a) Menschlich-gestalterische Tätigkeit 144

b) Geistiger Gehalt 145

c) Wahrnehmbarkeit 145

d) Individualität 145

3. Ergebnis 147

IV. Zusammenfassung 147

C. Trainierter Random Forest in $R \quad 148$

D. Trainierte Parameter 150

I. Schutz als Datenbankwerk gem. §4 Abs. 2 UrhG 150

1. Datenbankwerk 150

a) Künstliches neuronales Netz 151

b) Random Forest 151

c) Zwischenergebnis 152

2. Persönliche geistige Schöpfung 152

a) ML-Modell in Python $\quad 152$

b) Random Forest in $R$

3. Ergebnis 154

II. Investitionsschutz gem. §§ 87a ff. UrhG 155

1. Vorliegen einer Datenbank 155

2. Investition 155

a) Gegenstand der Investition 155

aa) Berechnung bzw. Optimierung der Parameter 156

bb) Andere Investitionsgegenstände 159

cc) Zwischenergebnis 159 
b) Wesentlichkeit 160

3. Ergebnis 160

4. Bewertung und praktische Relevanz 160

III. Zusammenfassung 161

E. Hyperparameter 161

I. Schutz als Datenbankwerk gem. § 4 Abs. 2 UrhG 162

1. Datenbank 162

2. Persönliche geistige Schöpfung 162

3. Ergebnis 163

II. Investitionsschutz gem. §§ 87a ff. UrhG 164

1. Datenbank 164

2. Wesentliche Investition 164

3. Ergebnis 165

III. Zusammenfassung 165

F. Untrainiertes Modell 165

$\begin{array}{lll}\S 8 & \text { Ergebnis des dritten Teils } & 169\end{array}$

Vierter Teil: Schutz von KI-Erzeugnissen 171

§ 9 Schutzvoraussetzungen im Urheberrecht im Kontext von KIErzeugnissen

A. Urheber ist stets ein Mensch 173

B. Persönliche geistige Schöpfung 174

I. Menschlich-gestalterische Tätigkeit 175

1. Bekanntes Terrain: Zufallskunst bzw. Computerkunst 176

a) Zurechnungszusammenhang 177

b) Auswahltheorie in Abgrenzung zur Präsentationslehre 178

c) Strenge Auswahltheorie 178

d) Kombinierte Auswahltheorie 179

e) Externe Auswahltheorie 179

f) Zusammenfassung 180

2. Vom Bekannten zum Unbekannten: KI-Kunst 181

3. Wann liegt beim Einsatz von KI menschlich-gestalterisches Handeln vor? 182

a) Problemstellung 182

b) Andernorts am Beispiel von ANGELINA vorgeschlagene Herangehensweise 
c) Kritik an dieser Vorgehensweise 186

d) Lösungsvorschlag 186

II. Geistiger Gehalt 189

III. Wahrnehmbarkeit, Individualität 190

$\begin{array}{ll}\text { C. Zusammenfassung } & 190\end{array}$

$\S 10$ Wem gebührt ein möglicher Schutz? 193

A. Forschungsstand: Wer ist warum Urheber? 193

B. Vorgeschlagene Vorgehensweise zur Ermittlung des Urhebers 195

C. Verwendung des Flussdiagramms anhand von Beispielen 204

I. Google DeepDream 204

II. Google Bach Doodle 206

III. Edmond de Belamy 207

IV. GPT-3 209

D. Zusammenfassung 210

$\S 11$ Ergebnis des vierten Teils 213

Fünfter Teil: Computational Creativity - Kreative Künstliche Intelligenz?

$\S 12$ Intelligenz, Kreativität und ihre Schnittstellen zueinander und zum Recht

A. Intelligenz 217

I. Definition der Intelligenz? 218

II. Relevanz der Intelligenz im Recht 219

1. Zivilrecht 219

2. Strafrecht 220

III. Folgerung 221

B. Kreativität 222

I. Definition? 223

1. Etymologischer Ursprung von „Kreativität“ 223

2. Psychologie 224

3. Kognitionswissenschaft 225

a) Boden: P-Kreativität und H-Kreativität 225

b) McCormack/d'Inverno 226

c) Sloman: Intrinsische, expressive und
standardtestbasierte Kreativität 
d) Miller: Little-c- und Big-C-Kreativität 228

4. Andere Ansätze 229

5. Schlussfolgerung: Einführung von Kreativitätsprofilen 229

II. Relevanz der Kreativität im Recht 231

1. Analogien 232

2. Auslegungsmethodik 233

III. Folgerung 234

C. Verhältnis von Kreativität und Intelligenz und deren Bedeutung für das Urheberrecht 234

I. Grundsätzliche Überlegungen 234

1. Beschreibbarkeitsproblem 235

2. Konsequenzen 235

3. Versuch einer Gegenüberstellung 236

II. Bedeutung von Intelligenz und Kreativität im Urheberrecht 237

1. Kreativität und Intelligenz als Grundvoraussetzung im Urheberrecht? 238

2. Von künstlicher Intelligenz über künstliche Kreativität zur hypothetischen Urheberschaft 239

a) Menschlicher vs. maschineller Schöpfungsprozess 240

b) Entstehung einer Idee 240

c) Autonomie im Schöpfungsprozess 241

D. Zusammenfassung 242

$\S 13$ Computational Creativity 245

A. The Painting Fool 247

B. ANGELINA 249

C. Vergleich von ANGELINA und The Painting Fool anhand ihrer Kreativitätsprofile 250

D. Kritische und kreative urheberrechtliche Überlegungen zu
Computational Creativity

I. Zu den Kriterien in $\S 2$ Abs. 2 UrhG 251

II. Mögliche Ausgestaltung eines „Urheberrechts“ für nichtmenschliche Urheber 254

1. Durchsetzung durch gesetzlichen Vertreter 254

2. Leistungsschutzrecht de lege ferenda 255

3. Schlussfolgerung und Zusammenfassung 255

III. Hypothetische Urheberschaft 256

1. The Painting Fool 256

2. ANGELINA 257 
IV. Schlussfolgerung 258

V. Zusammenfassung 258

E. Fazit 259

$\S 14$ Ergebnis des fünften Teils 261

Sechster Teil: Gesamtergebnis und Ausblick 263

$\S 15$ Gesamtergebnis 265

A. Urheberrechtliche Relevanz des ML-Prozesses 265

B. Urheberrechtlicher Schutz von ML-Modellen 265

C. Urheberrechtlicher Schutz von Erzeugnissen generativer ML-Modelle

D. Computational Creativity 267

$\S 16$ Ausblick 269

Mail-Austausch mit Mike Cook, Projekt ANGELINA 283 


\section{Abbildungsverzeichnis}

2.1 Entscheidungsbaum 47

2.2 Random Forest 48

2.3 Künstliches neuronales Netz mit vier Schichten 48

3.1 Phasen des Machine Learning-Prozesses 64

3.2 Bild mit 4x4 Pixel, stark vergrößerte Darstellung 66

4.1 Änderungen der TDM-Regelungen 91

6.1 „Objektorientierung“ im HGB 101

6.2 Entstehung eines ML-Modells 106

6.3 Überblick über Speichervarianten in den den untersuchten Python-Frameworks und Bibliotheken 113

7.1 Einfaches Beispiel in Keras: Laden eines Modells aus .h5-Datei 139

7.2 Die ersten 20 Einträge eines Baumes in einem Random Forest 148

7.3 Beispiel eines einfachen Quellcodes zur Erstellung eines Modells mit Keras 166

$\begin{array}{lll}\text { 8.1 Ergebnisübersicht Modellschutz } & 170\end{array}$

10.1 Flussdiagramm Urheberschaft 196

10.2 DeepDream: Input und Output 205

12.1 Kognitionswissenschaft im Kontext 222

12.2 Kategorien der Kreativität 230

12.3 Kreativitätsprofil Patentrecht 231

12.4 Kreativitätsprofile Analogie und Auslegung 233

12.5 Kreativitätsprofil Urheberrecht 238

13.1 Kreativitätsprofile ANGELINA und The Painting Fool 250 
Abkürzungsverzeichnis

AI Artificial Intelligence

Computerprogramm-RL Richtlinie 2009/24/EG des Europäischen Parlaments und des Rates vom 23. April 2009 über den Rechtsschutz von Computerprogrammen

Datenbank-RL Richtlinie 96/9/EG des Europäischen Parlaments und des Rates vom 11. März 1996 über den rechtlichen Schutz von Datenbanken

DSM-RL Richtlinie (EU) 2019/790 des Europäischen Parlaments und des Rates vom 17. April 2019 über das Urheberrecht und die verwandten Schutzrechte im digitalen Binnenmarkt und zur Änderung der Richtlinien 96/9/EG und 2001/29/EG

GeschGeh-RL Richtlinie (EU) 2016/943 des Europäischen Parlaments und des Rates vom 8. Juni 2016 über den Schutz vertraulichen Know-hows und vertraulicher Geschäftsinformationen (Geschäftsgeheimnisse) vor rechtswidrigem Erwerb sowie rechtswidriger Nutzung und Offenlegung

InfoSoc-RL Richtlinie 2001/29/EG des Europäischen Parlaments und des Rates vom 22. Mai 2001 zur Harmonisierung bestimmter Aspekte des Urheberrechts und der verwandten Schutzrechte in der Informationsgesellschaft

KI Künstliche Intelligenz

KNN Künstliches neuronales Netz

ML Maschinelles Lernen / Machine Learning

MLaaS Machine Learning as a Service

NLP Natural Language Processing

TDM Text- und Data-Mining

WIPO World Intellectual Property Organization

Zudem wird hinsichtlich der Verwendung anderer Abkürzungen in dieser Arbeit verwiesen auf Kirchner, Abkürzungsverzeichnis der Rechtssprache, 9. Auflage 2018. 
Erster Teil

Einführung 


\section{$\S 1$ Einleitung}

„Kreative Maschinen“- schon diese zwei Worte muten widersprüchlich an, es könnte gar ein Oxymoron vermutet werden. Der Gegensatz scheint nicht größer sein zu können: Maschinen arbeiten stur vorgegebene und bis ins kleinste Detail definierte Aufgaben ab, während „Kreative“ - Maler, Schriftsteller, Bildhauer, Beschäftigte der „Kreativbranche“ und andere - danach streben, immer Neues und Unerwartetes und Ungewöhnliches zu schaffen. Dennoch mehren sich die Stimmen, die von „kreativer künstlicher Intelligenz“, „kreativen Algorithmen“ und „Roboterjournalismus“ berichten. ${ }^{1}$ Einige halten dagegen, Kreativität und Maschinen passen nicht zusammen, schließen sich gegenseitig aus, oder Kreativität sei den Menschen (und vielleicht noch den Tieren) vorbehalten. ${ }^{2}$

Mit den Methoden des Machine Learnings - also Ansätzen, Maschinen lernen zu lassen, sei es aus Beispielen oder im Rahmen des Versuch-undIrrtum-Prinzips - scheint sich ein Weg zu öffnen, Maschinen von der Anforderung vollständiger Programmierung im Voraus zu befreien und ihnen die Möglichkeit zu geben, sich veränderten Bedingungen anzupassen. Diese angestrebte Abkehr von der vollkommen vorbestimmten Abarbeitung menschlicher Anweisungen könnte Maschinen womöglich auch die Fähigkeit der Kreativität näherbringen, sei es im Rahmen des kreativen Problemlösens oder unter Umständen auch im Kontext der Erzeugung von Ergebnissen, die, wenn sie von einem Menschen geschaffen worden wären, als Werke im Sinne des Urheberrechts eingeordnet werden könnten. Ob die Resultate als Kunst bezeichnet werden können, wenn sie von Maschinen produziert

1 Vgl. z. B. folgende Medienberichte: Álvarez, Wenn Computer Kunst schaffen (Tagesspiegel online vom 09.03.2020); Epstein et al., iScience Nr. 23 2020; Vogel, Kreativität und Codes (sueddeutsche.de vom 16.09.2020); Kremp, Roboterjournalismus auf MSN online (Spiegel.de vom 30.05.2020); sowie die Ausführungen zu Roboterjournalismus in Habel, Roboterjournalismus; eine Zusammenstellung aktueller kreativer (?) Systeme präsentiert Miller, The Artist in the Machine; Internetadressen befinden sich der Lesbarkeit halber im Literaturverzeichnis.

2 Vgl. z. B. Blöchl, „KI ist nicht kreativ“ (sueddeutsche.de vom 27.11.2020); Schwab, 3 reasons why AI will never match human creativity (fastcompany.com vom 25.04.2019); Miller, Can machines be more creative than humans? (TheGuardian.com vom 04.03.2019). 


\section{$\S 1$ Einleitung}

werden, bleibt zu klären und ist stark von der gesellschaftlichen Interpretation und Bewertung des Kunstbegriffes abhängig.

Im Kontext selbstlernender und produzierender Systeme ergeben sich zahlreiche urheberrechtliche Fragestellungen. Diese reichen von der grundsätzlichen Frage, ob Maschinen Urheberrechte zugestanden werden könnten, bis zu praktischen Fragen in Bezug auf Schutzmöglichkeiten für solche vermeintlich kreativen Systeme und deren Erzeugnisse.

\section{A. Problemstellungen}

Im Jahr 2018 befasste sich bereits der Wissenschaftliche Dienst der Bundesregierung mit einigen dieser Fragestellungen. ${ }^{3}$ Ziel dieser Arbeit ist es, eine deutlich tiefergehende Betrachtung vorzunehmen und dabei besonderes Augenmerk auf die technische Umsetzung der Konzepte kreativer Maschinen in Form von Machine Learning-Algorithmen zu legen. Notwendigerweise wird dabei auch auf das Konzept der künstlichen Intelligenz (KI) einzugehen sein, die praktischen Ausführungen werden sich jedoch stets auf maschinelles Lernen als Anwendungsfall der KI beziehen.

Dabei stehen die folgenden Problemstellungen im Mittelpunkt:

- Werden im Rahmen des Trainings von Machine Learning-Modellen urheberrechtlich relevante Handlungen vorgenommen, und wenn ja, gibt es eine Schranke zugunsten des Trainings von Modellen maschinellen Lernens mit urheberrechtlich geschützten Werken (in etwa analog oder gar unter Anwendbarkeit der Text- und Data-Mining-Schranke)?

- Wie können - und sollten - Machine Learning-Modelle urheberrechtlich geschützt werden?

- Machine Learning-Modelle werden zunehmend auch zur Erzeugung möglicherweise urheberrechtlich geschützter Werke eingesetzt (Bilder, literarische Werke, Musik,...). . Die verwendeten Algorithmen werden immer komplexer, wodurch der Einfluss des menschlichen Inputgebers auf den Output immer geringer wird. Ist das, was im Rahmen des Einsatzes solcher generativer Modelle als Erzeugnis dieses Prozesses entsteht, noch dem Menschen zuzurechnen? Ist das Erzeugnis noch eine persönliche geistige Schöpfung, wenn der Schaffensprozess durch eine „Black Box“ geht, die der Mensch nicht vollständig versteht? Oder müssen solche

3 Deutscher Bundestag, Künstliche Intelligenz und Machine Learning. Eine urheberrechtliche Betrachtung. 
Erzeugnisse als urheberrechtslos, sprich gemeinfrei, verstanden werden? Welche Möglichkeiten gibt es, die Erzeugnisse dennoch zu schützen?

- Welche Zusammenhänge bestehen zwischen Kreativität und (Urheber-) Recht? Was ist Kreativität, und worin besteht der Zusammenhang mit dem Konzept der Intelligenz? Können Maschinen kreativ sein? Wie wirkt sich das auf die Urheberrechte aus?

Im Rahmen dieser Arbeit erfolgt also eine umfassende urheberrechtliche Betrachtung des Machine Learning-Prozesses samt entstehender Erzeugnisse, die aufgrund der starken Orientierung an der tatsächlichen Umsetzung von Machine Learning in der Praxis zur Unterstützung der Beantwortung urheberrechtlicher Fragen herangezogen werden kann. Diese Fragen können etwa ausgehen von einem Benutzer eines auf einer Internetseite bereitgestellten Machine Learning-Systems zur Erzeugung von Bildern oder Musik, der wissen möchte, ob er die Urheberrechte an dem damit erzeugten Ergebnis hat; oder auch von einer Softwareentwicklerin, die sich darüber vergewissern möchte, wie das aufwendig konzipierte und implementierte Machine Learning-Modell urheberrechtlich geschützt ist. Um diesen Praxisbezug herzustellen und die relevanten Technologien zu identifizieren, wurden im Rahmen der Abfassung dieser Arbeit immer wieder Gespräche mit Praktikern aus den entsprechenden Fachgebieten geführt.

\section{B. Gang der Arbeit}

Diese Arbeit wird sich dem Thema entsprechend der oben genannten vier Problemstellungen annehmen.

Zunächst erfolgt im ersten Teil eine knappe, übersichtsvermittelnde Einführung in die Themen der künstlichen Intelligenz und des maschinellen Lernens, allerdings ohne dabei tief auf die technischen Grundlagen einzugehen. Stattdessen sind diese jeweils an der Stelle behandelt, an der sie für das Verständnis vorausgesetzt werden.

Der zweite Teil befasst sich mit der Frage, inwiefern der Machine LearningProzess urheberrechtlich relevante Handlungen umfasst, und ob diese von Schrankenbestimmungen erfasst werden. Dabei wird sowohl die aktuelle Fassung des Urheberrechtsgesetzes berücksichtigt wie auch die zu erwartenden Änderungen durch die Umsetzung der DSM-Richtlinie 2021.

Der dritte Teil analysiert die urheberrechtliche Situation in der Entwicklung von Machine Learning-Systemen. Dabei erfolgt die Untersuchung nicht 


\section{$\S 1$ Einleitung}

abstrakt, sondern stets anhand für die Praxis relevanter Implementierungen von Machine Learning-Systemen. Besonderes Augenmerk liegt hier auf der Identifikation von Schutzgegenständen und der Bewertung im Rahmen des Computerprogramm- und Datenbankschutzes.

Daran logisch anschließend befasst sich der vierte Teil mit von Machine Learning-Systemen generierten Erzeugnissen und deren urheberrechtlicher Schutzfähigkeit. Insbesondere wird es darum gehen, den Zurechnungszusammenhang zwischen Urheber und Werk im Kontext der Anforderung einer menschlich-gestalterischen Tätigkeit zu untersuchen. Zudem wird ein Schema vorgeschlagen, das die Zusammensetzung gängiger Machine LearningModelle berücksichtigt und das dabei hilft, bei einer Vielzahl an dem Entstehungsprozess eines Machine Learning-Erzeugnisses Beteiligter einen Urheber zu ermitteln.

Der fünfte Teil wirft einen Blick über den juristischen Tellerrand hinaus auf Intelligenz und Kreativität und ihre - untrennbare? - Verbindung mit dem Menschen. Im Zentrum des fünften Teils steht die Kreativität, insbesondere in der Form der „Computational Creativity“. Zudem wird zu diskutieren sein, ob de lege ferenda ein Schutz hypothetisch autonom geschaffener Werke erforderlich ist oder erforderlich werden könnte.

\section{Grenzen der Arbeit}

Auch wenn diese Arbeit den Anspruch stellt, insbesondere in Bezug auf die Untersuchung des Schutzes der Machine Learning-Modelle einen starken Praxisbezug aufzuweisen, können doch nicht alle verfügbaren Technologien Berücksichtigung finden. Es mussten daher einige Technologien herausgegriffen werden, die als weit verbreitet und daher sehr praxisrelevant eingeordnet werden.

Zudem unterliegen diese Technologien rapidem technischen Wandel. Die diskutierten Frameworks werden regelmäßig aktualisiert, teilweise geht damit auch eine Änderung der technischen Abläufe und Funktionen einher. Diese Arbeit kann daher, wenn technische Details aus den Frameworks und Bibliotheken bzw. Anwendungsschnittstellen diskutiert werden, nur den während der Erstellung der Arbeit aktuellen Stand wiedergeben. Anhand dessen wird ein Bild gezeichnet, das auch auf zukünftige Entwicklungen anwendbar ist.

Des Weiteren beschränkt sich diese Arbeit auf die Bewertung nach dem deutschen Urheberrecht. Andere Schutzmöglichkeiten, etwa Patentschutz 
oder der Schutz der Geschäftsgeheimnisse, für Modelle oder auch für Erzeugnisse, wurden bewusst ausgeklammert, weil diese nicht in der angemessenen Tiefe hätten berücksichtigt werden können und teilweise auch andernorts bereits ausführlich behandelt worden sind. Es wird jedoch an den entsprechenden Stellen auf weiterführende Literatur hingewiesen. Gleiches gilt für die Berücksichtigung anderer Rechtsordnungen. 


\section{$\S 2$ Grundlagen}

\section{A. Künstliche Intelligenz}

Der Titel dieser Arbeit verwendet weder die Begriffe ,künstliche Intelligenz“ noch „Machine Learning“, sondern spricht schlicht von „kreativen Maschinen“. Der Begriff der „Maschinen“ sollte keine Assoziation mit einer großen, dampfbetriebenen Industriemaschine hervorrufen, sondern vielmehr einen Gegensatz zum Begriff des Natürlich-Menschlichen darstellen. Gemeint sind insbesondere „Machine Learning“-Systeme, die in der Regel dem Oberbegriff der künstlichen Intelligenz zugeordnet werden.

Inzwischen dürfte sich der Begriff der „,künstlichen Intelligenz“ (KI) auch im Umfeld der Rechtswissenschaft - weit verbreitet haben, wenn auch die Vorstellungen dazu noch sehr subjektiv und möglicherweise romantisiert oder mit Vorurteilen belegt sind, die vielfach von gefährlichem Halbwissen oder Hörensagen und Science Fiction-Medien erzeugt und befeuert werden.

Was ist also gemeint, wenn von künstlicher Intelligenz die Rede ist? Gleich zu Beginn ist es wichtig, Fiktion von Realität zu trennen. Was ist reine Fantasie? Was funktioniert heute schon? Und was scheitert noch am Stand der Technik, wäre aber theoretisch realisierbar? Um für den weiteren Verlauf der Arbeit eine grundlegende Diskussionsbasis zu schaffen, wird dieses Kapitel KI - losgelöst von rechtlichen Fragestellungen - beleuchten. Dazu erfolgt zunächst ein Überblick über das KI-Thema (und was diese Arbeit darunter versteht). Daran schließen einige Erklärungen zur Funktionsweise zweier betrachteter Technologien an. Das Kapitel schließt mit Ausführungen zu praktischen Anwendungen der genannten Methoden, wobei der Fokus auf solchen Anwendungen liegt, die potenziell urheberrechtlich relevante Handlungen nahelegen.

\section{Begriff der künstlichen Intelligenz}

Der Begriff der „künstlichen Intelligenz“ gibt Anlass zu der Frage, ob er einen offensichtlichen Gegensatz zur „natürlichen Intelligenz“ darstellen soll. Herberger setzt sich mit den Begriffen des Künstlichen und der Intelligenz ausführlich auseinander, und stellt dabei zunächst fest, dass eine direkte 
Übersetzung des aus dem Englischen stammenden Begriffs der „Artificial Intelligence“ in „künstliche Intelligenz“, hält man sich an die historische Bedeutung der Wörter, nicht so recht zu passen scheint. ${ }^{4}$ In dieser Arbeit wird auf die Repetition andernorts durchgeführter etymologischer Herleitungen $^{5}$ verzichtet und folgender, vereinfachter, intuitiver Definitionsansatz als Grundlage gewählt:

Als „künstlich“ versteht der Mensch alles, was nicht im Ursprung natürlich entstanden ist, also was nicht lebendig ist und nicht schlicht aus der Evolution dem natürlichen Lauf der Dinge folgt; ,künstlich“ ist also etwas, bei dessen Entstehung nachgeholfen wurde, genauer, was der Mensch vollständig - nicht im Wege der Fortpflanzung - erschaffen hat. ${ }^{6}$

Nach dieser Definition wäre „,künstliche Intelligenz“ eine vom Menschen gesteuerte oder entwickelte Intelligenz. Diese Annahme basiert allerdings darauf, dass der Begriff der ,künstlichen Intelligenz“ tatsächlich auf „Intelligenz" abstellt und nicht eigentlich auf etwas ganz anderes abzielt. ${ }^{7}$ Wie auch später noch festzustellen sein wird, ist auch der Begriff der Intelligenz nicht leicht zu fassen. Was also meint künstliche Intelligenz?

\section{Ursprung}

Um diese Frage zu beantworten, erscheint es hilfreich, die Ursprünge der KI(-Forschung) zu erkunden. Die offiziellen Anfänge der Wissenschaft um künstliche Intelligenz liegen wohl im Jahr 1956 am Dartmouth College in Hanover, New Hampshire, USA. ${ }^{8}$

Die Idee, den Menschen - bzw. menschliche Gedankenvorgänge - künstlich nachzubauen, ist jedoch viel älter und naturgemäß eng mit der Entwicklung der Computer verbunden. ${ }^{9}$ Von Maschinen, die dem Menschen lästige Arbeit abnehmen - sei es, weil hierzu großer Muskelkraftaufwand oder umfangreiche Denkleistung erforderlich ist - ist der Sprung zu solchen Automaten, die mitunter Sparringspartner im intellektuellen Duell sein könn-

4 Herberger, NJW 2018, 2825, 2826 f..

5 Ders., NJW 2018, $2825 \mathrm{ff}$. .

6 Vgl. z. B. https://www.duden.de/rechtschreibung/kuenstlich (Stand: 22.02.2021).

7 Dies bezweifelt auch Herberger, NJW 2018, 2825 f..

8 McCarthy et al., Dartmouth Conference Proposal 1955.

9 Einen ausführlichen chronologischen Überblick über die Anfänge der KI samt Einordnung in die Computergeschichte gibt McCorduck, Machines Who Think, S. XXIII ff.. 
ten, naheliegend (und doch - aus der Perspektive der Anfangszeit - scheinbar unerreichbar).

So kommentierte Ada Lovelace bereits 1843 eine Version der „Analytical Engine" von Charles Babbage, ${ }^{10}$ und entwickelte darin für damalige Zeiten sehr futuristische Gedanken:

„Supposing, for instance, that the fundamental relations of pitched sounds in the science of harmony and of musical composition were susceptible of such expression and adaptations, the engine might compose elaborate and scientific pieces of music of any degree of complexity or extent." ${ }^{\text {"11 }}$

Hier wird allerdings deutlich, dass weniger Bestrebungen im Mittelpunkt stehen, die menschliche Intelligenz oder die Struktur des Gehirns künstlich nachzubauen, sondern vielmehr eine wissenschaftliche Basis und Berechenbarkeit - in diesem Falle der Musik - zu erforschen, um daraus möglicherweise Regeln aufzustellen, die dann wiederum programmatisch einen Computer „komponieren“ lassen könnten. Auch wenn hier noch kein direkter Bezug zur Schaffung künstlicher Intelligenz bestand, so sind diese Gedanken doch Zeugnis dafür, dass der Mensch schon früh Vorstellungen davon hatte, wie klassischerweise dem menschlichen Verstand vorbehaltene Vorgänge maschinell abgebildet werden könnten.

1943 veröffentlichen $M c$ Culloch und Pitts ihre Ausarbeitung „A Logical Calculus of the Ideas Immanent in Nervous Activity “12, in der sie sich mit der logischen Abbildung neuronaler (Gehirn-)Aktivitäten beschäftigen und umgekehrt feststellen, dass für jede logische Operation ein diese abbildendes Netz gefunden werden kann. Der Bezug zur späteren KI-Forschung ist offensichtlich, insbesondere bei Betrachtung künstlicher neuronaler Netze als Modelle des maschinellen Lernens.

10 Die Analytical Engine ist ein früher Entwurf einer mechanischen Rechenmaschine, Babbages Notizen dazu sind z. B. hier einsehbar: https://collection. sciencemuseumgroup.org.uk/documents/aa110000020 (Stand: 22.02.2021).

11 Lovelace, Scientific Memoirs 1843, 666, 694, Volltext abrufbar unter https://www. fourmilab.ch/babbage/sketch.html (Stand: 22.02.2021), übersetzt: „Angenommen, beispielsweise, dass die fundamentalen Beziehungen von Tönen in der Wissenschaft von Harmonie (Harmonielehre) und musikalischer Komposition fähig wären zu solchem Ausdruck und Anpassungen [wie etwa Nummern im Kontext der abstrakten Wissenschaft von Rechenoperationen], dann könnte die Maschine elaborierte und wissenschaftliche Musikstücke jeglichen Grades an Komplexität oder Ausmaß komponieren."

12 McCulloch/Pitts, The Bulletin of Mathematical Biophysics Band 5 Nr. 4 1943, 155 ff.. 
Kurz darauf verfasst Alan Turing „Intelligent Machinery“13, ein Papier, das jedoch nie als solches veröffentlicht wurde. Auch Turing orientiert sich darin an der Struktur des menschlichen Gehirns und weist bereits darauf hin, dass auch für die Maschine ein Lernprozess unumgänglich sei, ${ }^{14}$ eine Erkenntnis, die heute noch im Kontext des Machine Learnings zentral für die Umsetzung „künstlicher Intelligenz“ ist.

Auch Isaac Asimov's berühmte drei Gesetze der Robotik ${ }^{15}$ stammen aus der Zeit vor den Anfängen der KI-Wissenschaft: Sein Buch „I, Robot“ erschien bereits 1950, und damit im selben Jahr, in dem auch Turing seinen Turing Test publiziert. ${ }^{16}$

Aus dem „Proposal for the Dartmouth Summer Research Project on Artificial Intelligence“ von McCarthy, Minsky, Rochester und Shannon aus dem Jahr 1955 geht hervor, dass die KI-Forschung sich mit Lernprozessen auseinandersetzen soll. Die vorgeschlagene Studie basiert (wie ja ähnlich auch schon von Ada Lovelace mehr als einhundert Jahre zuvor formuliert) auf der Annahme, dass jeder Lernprozess so exakt definiert bzw. beschrieben werden kann, dass eine Maschine ihn nachzuahmen in der Lage ist. ${ }^{17}$

Auch wenn das Projekt wohl aus Gründen mangelnden Commitments der beteiligten Wissenschaftler nie so stattfinden konnte wie geplant, hat es gleichwohl den Namen der Wissenschaft geprägt ${ }^{18}$ und den sozialen Grundstock an personellen Verbindungen zum effizienten Wissensaustausch gelegt. ${ }^{19}$ Es

13 Turing, Intelligent Machinery, $107 \mathrm{ff.}$.

14 ,It is pointed out that the potentialities of the human intelligence can only be realized if suitable education is provided. The investigation mainly centres round an analogous teaching process applied to machines.“ Ders., Intelligent Machinery, 107.

15 Die Gesetze lauten:

„1. Ein Roboter darf kein menschliches Wesen (wissentlich) verletzen oder durch Untätigkeit (wissentlich) zulassen, dass einem menschlichen Wesen Schaden zugefügt wird.

2. Ein Roboter muss den ihm von einem Menschen gegebenen Befehlen gehorchen es sei denn, ein solcher Befehl würde mit Regel eins kollidieren.

3. Ein Roboter muss seine Existenz beschützen, solange dieser Schutz nicht mit Regel eins oder zwei kollidiert.“,Asimov, I, Robot, S. 44 f., Übersetzung aus dem Englischen: Ders., Meine Freunde, die Roboter: Erzählungen, S. 67.

16 Turing, Mind LIX Nr. 49 1950, 433 ff..

17 McCarthy et al., Dartmouth Conference Proposal 1955.

18 McCorduck, Machines Who Think, S. 114, andere gängige Begriffe für Forschung auf dem Gebiet - im Jahr 1956 - waren etwa „Automata Studies“, Shannon/McCarthy, Automata Studies. (AM-34), oder „Complex Information Processing“, McCorduck, Machines Who Think, S. 115.

19 Dies., Machines Who Think, S. 130. 
klingt also bereits an, dass „Artificial Intelligence“ weniger die direkte Nachahmung der menschlichen Intelligenz meint, ${ }^{20}$ sondern vielmehr erforscht werden soll, wie der - bis dato dem Lebewesen vorbehaltene - Lern- bzw. Problemlösungsprozess maschinell modelliert werden kann.

\section{KI heute}

Nicht nur die Technik, sondern auch das Forschungsgebiet der KI hat sich seit den 1950er Jahren weiterentwickelt. Es sind Teilgebiete entstanden: Computer Vision, Robotik, Sprachverarbeitung (Natural Language Processing NLP), Entscheidungstheorien und insbesondere maschinelles Lernen. ${ }^{21}$ Bis heute ist unklar, wie „künstliche Intelligenz“ tatsächlich zu definieren ist (was auch daran liegen mag, dass selbst die natürliche Intelligenz mit ihrer Definierbarkeit zu kämpfen hat). ${ }^{22}$

Möglicherweise liegt die Herausforderung, KI zu definieren, auch darin, dass KI sich regelmäßig selbst „erledigt“: Sobald ein maßgeblicher Erfolg erzielt wird, also eine Aufgabe oder ein Problem gelöst wird, das scheinbar nur mit KI zu lösen ist, wird diese Lösung schnell zum Standard, und die Messlatte für künstliche Intelligenz steigt. ${ }^{23}$ Sobald der Mensch erkennt, wie das Problem gelöst wird, verschwindet der ,magische Effekt“ der Intelligenz; „Das kann dann keine Intelligenz mehr sein“, „Das ist ja einfach nur ein Computerprogramm“, wird dann oft gesagt, weshalb viele Fortschritte, die der KI-Forschung zuzuschreiben sind, nicht die Anerkennung bekommen, die sie verdienen. ${ }^{24}$ Wenn wir jedoch alles, was - sobald erreicht - als ultimative künstliche Intelligenz gelten soll, nach der tatsächlichen Implementierung als reines Werkzeug abtun, ist es kein Wunder, dass es unmöglich scheint, $\mathrm{KI}$ abschließend zu definieren. Und so ist es auch nachvollziehbar, dass sich die Definition, was künstliche Intelligenz ausmacht, ständig verändert und nur punktuell fassen lässt.

20 So sieht es allerdings wohl Zech, ZfPW 2019, 198, 199, der dies auch als das Verständnis der Dartmouth Conference auffasst.

21 McCorduck, Machines Who Think, S. 417.

22 Dies., Machines Who Think, S. 419; Craglia et al., Artificial intelligence: A European perspective, S. 19; vgl. dazu außerdem auch $\S 12$ A..

23 So auch McCorduck, Machines Who Think, S. 423, Kaplan, Artificial intelligence: What everyone needs to know, S. 37.

24 Vgl. auch McCorduck, Machines Who Think, S. 423. 


\section{a) Definitionsansätze}

Gleich vorweg: diese Arbeit erhebt weder den Anspruch, eine neue, uneingeschränkt gültige Definition für KI aufzustellen, noch will sie alle jemals erdachten Definitionen wiedergeben. Vielmehr erfolgt an dieser Stelle eine Destillation des bisher andernorts Gesagten (soweit dies unter Berücksichtigung der schieren Menge der Definitionsversuche möglich ist), um eine für das weitere Vorgehen gangbare Diskussionsgrundlage zu schaffen. Aufbauend auf den Grundlagen und Anfängen der KI-Wissenschaft, die soeben dargelegt wurden, wird im Folgenden ausgegangen von den „klassischen Definitionsansätzen“. Sodann werden alternative Vorschläge kurz angerissen, um schlussendlich bei einer strukturellen Definition zu landen.

aa) „Klassische“ KI-Definitionen

Definitionen aus der Anfangszeit der KI beschäftigen sich mit dem Kerngebiet der algorithmischen Umsetzung bzw. Nachahmung menschlicher Intelligenz. So wurde im Dartmouth-Konferenz-Proposal (1955) das „Künstliche Intelligenz-Problem“ beschrieben als ,die Aufgabe, eine Maschine dazu zu bringen, sich so zu verhalten, dass, würde ein Mensch sich so verhalten, diese Verhaltensweise als intelligent bezeichnet würde“ ${ }^{25}$

Minsky stellt bereits im Vorwort zu „Semantic Information Processing“ (1968) klar, dass Künstliche Intelligenz (bzw. Artificial Intelligence) die „Wissenschaft [ist], Maschinen dazu zu bringen, das zu tun, was Intelligenz erfordern würde, wenn es durch Menschen getan würde“"26 und liegt damit sehr nah an der Definition der Dartmouth-Konferenz: Auch hier wird für einen Vergleich bzw. den Maßstab des Erreichens künstlicher Intelligenz der Mensch herangezogen.

Russell/Norvig scheinen einen anderen Definitionsansatz zu verfolgen: Sie beschreiben „das Gebiet der KI“ als den Versuch, ,den Beweis für das Verständnis [von Intelligenz] zu führen, indem es intelligente, technische Systeme erschafft" ${ }^{27}$ und schlüsseln sodann vier Bereiche auf, denen sie

25 McCarthy et al., Dartmouth Conference Proposal 1955; Ertel, Grundkurs Künstliche Intelligenz: Eine praxisorientierte Einführung, S. 1.

26 Minsky, Semantic Information Processing, S. V.

27 Russell/Norvig, Künstliche Intelligenz, S. 22. 
bestehende Definitionen zuweisen: Menschliches Denken, rationales Denken, menschliches Handeln und rationales Handeln. ${ }^{28}$

Kaplan (2016) hingegen definiert „,die Essenz der KI“ als die Fähigkeit, in einem angemessenen zeitlichen Rahmen passende generalisierende Schlüsse auf der Grundlage begrenzter Daten zu ziehen, ${ }^{29}$ und führt dann weiter aus, dass Lernen ein Prozess des Generalisierens sei, bei dem frühere Erfahrungen in zukünftige Analysen einbezogen werden. ${ }^{30}$ Auffällig ist: hier wird kein Vergleich mehr zu menschlichen Fähigkeiten oder dem Menschen insgesamt gezogen.

Kaplan stellt allerdings auch fest, dass dies nicht nur die „Essenz der KI“ beschreibt, sondern gleichfalls die „Essenz der Intelligenz““ ${ }^{31}$ Er unterscheidet also gar nicht mehr zwischen der menschlichen und der künstlichen Intelligenz, sondern wählt eine abstrakte Definition, die es dann später technisch umzusetzen gilt, nur um dann wenig später zu konstatieren:

„We may not be able to define AI just yet, but in the meantime I'm confident that most people feel, as U.S. Supreme Court justice Potter Stewart famously said of pornography, 'I know it when I see it'. “32

Die WIPO (2020) definiert KI in ihrem „Revised Issues Paper“" vom 21.05.2020 ähnlich wie Minsky, nämlich als „Disziplin der Computerwissenschaften, die darauf abzielt, Maschinen und Systeme zu entwickeln, die Aufgaben ausführen können, von denen angenommen wird, dass sie menschliche Intelligenz erfordern, ohne oder mit nur eingeschränkter menschlicher Intervention. “33

Auch denkbar ist eine Umformung des englischen Akronyms AI in „Augmented Intelligence“, also „verbesserte“ oder „erweiterte“ Intelligenz, wobei auch hier die Interpretationen in verschiedene Richtungen gehen: So könnte Augmented Intelligence als eine Beschreibung der Partnerschaft zwischen Mensch und Maschine verstanden werden, ${ }^{34}$ oder andererseits als zweite Stu-

28 Russell/Norvig, Künstliche Intelligenz, S. 23.

29 Kaplan, Artificial intelligence: What everyone needs to know, S. 5.

30 Ders., Artificial intelligence: What everyone needs to know, S. 6.

31 Ders., Artificial intelligence: What everyone needs to know, S. 5.

32 Ders., Artificial intelligence: What everyone needs to know, S. 7, Nachweis zu dem Zitat des Richters dort in Fn. 10.

33 WIPO, Revised Issues Paper, S. 3: „Artificial Intelligence (AI) is a discipline of computer science that is aimed at developing machines and systems that can carry out tasks considered to require human intelligence, with limited or no human intervention."

34 Hurwitz et al., Augmented Intelligence: The Business Power of Human-Machine Collaboration, S. 2. 
fe dreier Reifestadien von KI (Assisted Intelligence, Augmented Intelligence, Autonomous Intelligence) $)^{35}$.

Sascha Lobo stellt schließlich 2019 fest, im Alltag ließe sich KI ,synonym $\mathrm{zu} »$ lernende Mustererkennung « verwenden“"36. Ein Bezug zum Begriff der Intelligenz wird hier gar nicht mehr hergestellt, die Definition klingt vielmehr wie eine (zu kurz greifende, aber in der aktuellen Praxis wohl sehr treffende) Reduktion von KI auf maschinelles Lernen.

Für die ,klassische“ Definition des Begriffs der KI gestaltet sich die Einigung auf einen allgemeinen Begriff erkennbar schwierig.

\section{bb) Alternative Definition}

Die oben (§ 2 A.I.2.) genannten und heute zur KI gezählten Teilgebiete der KI betrachtend drängt sich der Gedanke auf, ob das, was erreicht werden soll, überhaupt noch unter den Begriff der Intelligenz zu fassen ist. Schließlich soll diese Wissenschaft scheinbar nicht nur das erforschen und nachzumodellieren versuchen, was im Inneren des menschlichen Gehirns vorgeht, sondern auch Robotik (also der nach außen sichtbare, mechanische Teil, der notwendig ist, um einen tatsächlichen Menschen zu simulieren), Computer Vision (also das Sehvermögen) und die Sprachverarbeitung (als Kommunikationsmöglichkeit) umfassen.

Eventuell wäre es also passender, das Gesamtgebiet der „,künstlichen Intelligenz“ mit seinen Teilgebieten eher als „Lebewesensimulation“, „Artificial Life“ oder „Artificial Human“ zu bezeichnen, und die künstliche Intelligenz auf das zu beschränken, was tatsächlich im Kopf vorgehen soll. Schließlich funktioniert das Gehirn auch ohne die Möglichkeit, zu sehen, zu sprechen, zu hören, oder Körperteile zu bewegen (obgleich ein Umkehrschluss nicht funktionieren mag).

cc) Hier: Strukturelle Definition

Ein eher nüchterner, jedoch auch in dieser Arbeit vertretener Ansatz betrachtet KI als Oberbegriff für die oben genannten Teilgebiete (Robotik, Computer

35 Mohanty/Vyas, How to Compete in the Age of Artificial Intelligence: Implementing a Collaborative Human-Machine Strategy for Your Business, S. 13.

36 Lobo, Realitätsschock, S. 221. 
Vision etc.) denen auch das maschinelle Lernen zuzuordnen ist. ${ }^{37}$ Durch diese strukturelle Sicht auf künstliche Intelligenz nähert sich der Begriff der Realität: Es wird in zahlreichen Teildisziplinen versucht, menschliche Fähigkeiten nachzubilden. Und das, was im Rahmen dieser Arbeit behandelt wird - der „Denkvorgang“"oder „Lernprozess“, wenn man so will - begründet das Forschungsgebiet des maschinellen Lernens. Angesichts dessen, was auch schon die „Urmütter“ und „Urväter“ der KI erreichen wollten, ${ }^{38}$ scheint dieser Begriff ohnehin passender. Diese Arbeit befasst sich mit dem, was in Code und Daten gefasst werden kann, also mit dem, was ,im Kopf der Maschine vorgeht", weshalb vorwiegend eine Beschreibung als maschinelles Lernen erfolgt. Wenn in den folgenden Kapiteln dennoch einmal von KI die Rede ist, so ist darunter - hier - maschinelles Lernen zu verstehen.

\section{b) Funktionsmäßige Kategorisierung}

Sobald eine gangbare Definition gefunden wurde, erfolgt üblicherweise eine funktionale Unterteilung nach der Leistungsfähigkeit von KI-Systemen. Unterschieden wird nach schwacher und starker KI, ${ }^{39}$ oft auch spezifische und generelle KI genannt. ${ }^{40}$ Diese Zuordnung ist nicht ganz unumstritten. Die Begriffe ,stark“ und ,schwach“ werden im KI-Umfeld auch eingesetzt, um zwischen zwei Perspektiven auf KI zu unterscheiden: Die eine Seite ist überzeugt davon, dass KI-Maschinen bereits jetzt oder in der Zukunft einen „Geist" haben werden, während die andere auf dem Standpunkt steht, dass KI-Technologien Intelligenz weniger duplizieren als simulieren. ${ }^{41}$ Zudem veranschaulicht diese Unterscheidung in sich auch die Hoffnung, vielleicht auch das Ziel der KI-Forschung, eine generelle, omnipotente KI zu schaffen,

37 So auch EC HLEG AI, A definition of AI, 7; Herberger, NJW 2018, 2825, 2827; Linke, GRUR Junge Wissenschaft 2019, S. 30 und S. 37; ebenfalls auf eine konkrete Definition verzichtend und auf Gebiete bzw. Elemente zurückgreifend Kaulartz/ Braegelmann, Rechtshandbuch Artificial Intelligence, Kap. 1 Rn. 9.

38 Vgl. aa).

39 So auch Kaulartz/Braegelmann, Rechtshandbuch Artificial Intelligence, Kap. 1 Rn. 10; ebenso WIPO, Revised Issues Paper, S. 3 f..

40 Unterscheidung nach stark und schwach geht zurück auf Searle, The Behavioral and Brain Sciences 3 1980, 417; ebenfalls Ausführungen zur Unterscheidung machen u. a. Lenzen, Natürliche und künstliche Intelligenz, S. 15; Datenethikkommission der BReg, Gutachten der Datenethikkommission der Bundesregierung, S. 59; Koncsik, Quantum Mind, S. 17; Specht-Riemenschneider, FS Taeger, 711, 713.

41 Kaplan, Artificial intelligence: What everyone needs to know, S. 68. 
und stellt dem die Realität gegenüber: Der letzteren Kategorie der „starken“ KI dürften wohl bis dato noch keine Systeme angehören. Vielerorts wird eine Kategorisierung dieser Art vorgenommen, für die Zwecke dieser Arbeit ist diese Unterscheidung nicht relevant und wird daher nicht weiter erläutert.

\section{Zusammenfassung}

Um die zu Beginn des Kapitels aufgeworfene Frage zu beantworten: Nein, „künstliche Intelligenz“ soll - zumindest in dieser Arbeit - keinen Gegensatz zum Begriff der ,natürlichen Intelligenz“ darstellen, sondern nur als Oberbegriff verschiedener Disziplinen verstanden werden, von denen besonders eine - das maschinelle Lernen - im Fokus der Betrachtung stehen wird.

\section{B. Maschinelles Lernen}

Ein Thema, dass schon zu Dartmouth-Zeiten ${ }^{42}$ untersucht wurde, und nach wie vor große Aufmerksamkeit bekommt, ist das Modellieren von Lernprozessen, und damit verbunden der quasi selbständige Wissensaufbau durch Algorithmen. Dieses Feld wird heute unter dem Oberbegriff des maschinellen Lernens gefasst. Es gehört ebenso zu den Anfängen der KI, und seine Erforschung hat sogar bereits vor der Dartmouth-Konferenz begonnen: Maschinelles Lernen geht wohl zumindest zurück auf Warren McCulloch und Walter Pitts und deren Forschung in 1943, auch wenn das maschinelle Lernen bis in die 1990-er Jahre nicht vorrangig erforscht wurde, ${ }^{43}$ was auch an der sehr begrenzten zur Verfügung stehenden Rechenkraft und Speicherkapazität gelegen haben könnte. ${ }^{44}$ Die Haupttreiber dieses Forschungsfeldes waren die Steigerung dieser technischen Möglichkeiten, die zunehmende Digitalisierung bzw. digitale Erfassung von Daten, einfacherer Zugang zu denselben (v. a. unterstützt durch das Internet) sowie günstige hochauflösende digitale Sensoren. ${ }^{45}$

42 Vgl. aa).

43 Kaplan, Artificial intelligence: What everyone needs to know, S. 32.

44 Ders., Artificial intelligence: What everyone needs to know, S. 39; Goodfellow et al., Deep Learning Handbuch, S. 20.

45 Kaplan, Artificial intelligence: What everyone needs to know, S. 39. 


\section{Definitionen}

Zunächst eine grundlegende Begrifflichkeitsklärung: „Maschine“ in „,maschinelles Lernen“ meint nicht zwangsläufig eine „Maschine“ im klassischen Sinne. Mit „Maschine“ wird oft ein mechanisches Gerät assoziiert, das mehr oder weniger automatisiert - etwa in der Produktion von Gegenständen eingesetzt wird, oder aber zunehmend auch Computer im Hardwaresinne. Im Kontext von „Maschinellem Lernen“ bzw. „Machine Learning“ zielt „Maschine“ aber wohl eher in die Software-Richtung: So wurde der Begriff bereits in den 50er-Jahren im KI-Kontext vor allem als Synonym für „Programm" verwendet, mutmaßlich daraus folgend, dass Turing seine abstrakten Prozeduren bereits als „Maschine“ bezeichnete. ${ }^{46}$

Es geht also um lernende Algorithmen, oder auch um Lernen durch Algorithmen (denn von einem lernenden Algorithmus könnte erwartet werden, dass er sich verändert - und das ist nicht immer der Fall).

\section{Algorithmus}

Algorithmen lassen sich definieren als ,Verfahren zur schrittweisen Umformung von Zeichenreihen“ oder als „Rechenvorgang nach einem bestimmten, sich wiederholenden Schema", ${ }^{47}$ allerdings wird der Begriff häufig auch verwendet, wenn von Computerprogrammen die Rede ist. Jedoch sollten „Computerprogramm“ und „Algorithmus" nicht synonym verwendet werden, denn ein Algorithmus (als allgemeine Regel zur Lösung eines Problems) kann durch verschiedenste Computerprogramme in eine für einen Computer verständliche Sprache umgesetzt werden. In dieser Arbeit werden Algorithmen in ihrer eigentlichen Form nicht tiefgehend thematisiert, stattdessen werden Computerprogramme behandelt (hier häufig kurz „Code“ genannt).

\section{Lernen}

Der Machine Learning-Forscher Goodfellow definiert Lernen als das „Mittel zum Erlangen der Fähigkeit, die für das Durchführen der Aufgabe benötigt

46 McCorduck, Machines Who Think, S. 119.

47 https://www.duden.de/rechtschreibung/Algorithmus (Stand: 22.02.2021). 
wird““ ${ }^{48}$ Lernen ist für ihn also ein Werkzeug - möglicherweise in Form eines Algorithmus - das eingesetzt werden kann, um eine Fähigkeit zu erwerben, die zur Zielerreichung erforderlich ist. Da es hier nur um das Lernen im Kontext von Machine Learning geht, werden Definitionen etwa aus dem Gebiet der Pädagogik hier nicht berücksichtigt.

\section{Machine Learning}

Machine Learning hingegen beschreibt Goodfellow als den Versuch, ,Regeln zu finden, die vermutlich für die meisten Elemente der betrachteten Menge korrekt sind““ ${ }^{49}$ Hieraus lässt sich schon erkennen, dass Machine Learning vor allem Mustererkennung ist, präziser formuliert: Das Erlernen der Fähigkeit, Muster zu erkennen.

Der Herausforderung, dem Computer - bzw. dem Algorithmus - das Lernen beizubringen, näherte man sich von unterschiedlichen Seiten. In den letzten Jahren haben sich dabei einige Standards herausgebildet, die im Folgenden zunächst überblicksmäßig dargestellt und anschließend näher erklärt werden. Mit den dargestellten Ansätzen soll das System lernen, Antworten zu geben, oder konkreter: Muster zu erkennen, zum Beispiel, indem ein Objekt auf einem Bild erkannt (,Was siehst du hier?“, könnte die Frage lauten, auf die das Programm die Antwort geben soll) oder das Alter einer Person geschätzt wird (hier würde dann konkret gefragt: „Wie alt ist diese Person?“). Dabei wird grundsätzlich unterschieden zwischen Klassifikation und Regression, sowie überwachtem, unüberwachtem und verstärkendem Lernen. ${ }^{50}$

\section{Modell}

Für diese Arbeit ist der Begriff des Modells wesentlich, insbesondere erfolgt die Verwendung im Rahmen von „Machine Learning-Modellen“ sehr häufig. Der Vielfalt an möglichen Bedeutungen dieses Begriffes halber erfolgt an dieser Stelle eine für diese Arbeit geltende Konkretisierung.

Die grundlegende Bedeutung des Begriffs ist nach Brockhaus zweigeteilt: Zum einen kann „Modell“ einen Entwurf oder ein Muster meinen, zum

48 Goodfellow et al., Deep Learning Handbuch, S. 109.

49 Dies., Deep Learning Handbuch, S. 130.

50 Vgl. auch Dies., Deep Learning Handbuch, S. 108, 115. 
anderen kann „Modell“ aber auch in der Bedeutung „Vorbild, Beispiel“ verwendet werden. ${ }^{51}$ Die Wortherkunft liegt im italienischen modello bzw. im lateinischen modulus (Maß, Maßstab). ${ }^{52}$

Unterschieden wird also zwischen der Bedeutung, die als „Modell“ das Original versteht, also einen Gegenstand, der im Betrachtungsmittelpunkt steht, und der nachgebildet oder bearbeitet wird, und andererseits der Vorstufe eines zu schaffenden Ergebnisses. Beide Bedeutungen liegen nahe an dem, was der Modellbegriff im Machine Learning meint, aber treffen es nicht ganz. Hierzu ist eine Definition aus dem Machine Learning-Kontext hilfreicher: Im Google Machine Learning Glossary wird Modell definiert als eine „Repräsentation dessen, was ein Machine Learning-System aus Trainingsdaten gelernt hat. “"53

Diese Repräsentation ist jedoch nicht als ein abstraktes, undurchschaubares Konstrukt zu verstehen, sondern vielmehr als ein konkretes Gebilde, das aus Zahlenwerten und Funktionsinformationen zusammengesetzt ist. Dabei gibt es Modellarten, die sich etabliert haben - wie etwa künstliche neuronale Netze oder Random Forests - die den Entwicklern zur Auswahl stehen, und die sie dann anpassen, trainieren und evaluieren. Das Endergebnis des Entwicklungsprozesses im Machine Learning, also das Gebilde, das eingesetzt wird, um Vorhersagen auf neuen Daten zu treffen, Daten zu sortieren oder Gemälde zu erzeugen, heißt (immer noch) Modell. Die Formen, die das ML-Modell annehmen kann, werden in $\S 6 \mathrm{D}$. beschrieben.

\section{Untersuchte Technologien}

Freilich wird hier nur auf einen kleinen Ausschnitt heute verfügbarer Technologien eingegangen, nämlich auf diejenigen, die im weiteren Verlauf der Arbeit zum Verständnis der Argumentation hilfreich sind: Entscheidungsbäume bzw. Random Forest-Modelle und künstliche neuronale Netze. ${ }^{54}$

51 Vgl. dazu den Brockhaus-Eintrag „Modell“ in https://brockhaus.de/ecs/enzy/article/ modell-allgemein (Stand: 22.02.2021).

52 Vgl. Brockhaus „Modell“, https://brockhaus.de/ecs/enzy/article/modell-allgemein (Stand: 22.02.2021).

53 Vgl. Google Machine Learning Glossary, https://developers.google.com/machinelearning/glossary\#model (Stand: 22.02.2021).

54 Die folgenden Ausführungen in Abschnitt § 2 B.II.1. und $\S 2$ B.II.2. wurden teilweise bereits abgedruckt in Kädelvon Maltzan, CR 2020, $66 \mathrm{ff.}$. 
1. Entscheidungsbäume bzw. Random Forest-Modelle

Schon der Name „Random Forest“ verspricht zumindest irgendein Zufallselement bei der Ermittlung der Antwort auf die gestellte Frage. Diese Systeme sagen zum Beispiel die Wahrscheinlichkeit einer bestimmten Antwort auf eine datenbezogene Frage voraus, indem sie relevante Eigenschaften der Datensätze identifizieren und deren Beitrag zum Ergebnis berechnen. Random Forest-Modelle ${ }^{55}$ basieren auf Entscheidungsbäumen. ${ }^{56}$ In einer Baumstruktur mit je zwei Zweigen - Ja/Nein, Wahr/Falsch, 1/0 - durchläuft ein Datensatz mehrere Entscheidungsknoten von oben nach unten. ${ }^{57}$

Oft wird zur Veranschaulichung das Beispiel der Überlebenswahrscheinlichkeit von Passagieren auf der Titanic herangezogen. Je nach Geschlecht, Reiseklasse, Alter und Anzahl der Familienmitglieder könnte vorhergesagt werden, wie wahrscheinlich eine fiktive Person den Untergang der Titanic überlebt hätte. Ein Entscheidungsbaum-Machine Learning-Algorithmus ${ }^{58}$ (,Modell“) erhält Datensätze mit Label - im Beispiel Passagiere mit Angabe über Überleben oder Sterben - und identifiziert aus dieser Vielzahl von Datensätzen anhand von Statistik die relevantesten Entscheidungskriterien (Features) und Schwellenwerte für die Entscheidungsknoten (,das Modell wird trainiert"). Nach den Schwellenwerten entscheidet sich, ob der Datensatz zum rechten oder linken Kindknoten weiter wandert. Der Prozess wiederholt sich für jeden neuen Entscheidungsknoten. Der Baum baut sich also sukzessive in eine Richtung selbst auf. Die Features ergeben sich aus den zur Verfügung stehenden Daten (beispielsweise Alter, Geschlecht, Reiseklasse). Am Ende des Baumes - in Abbildung 2.1 als „A“ und „B“ gekennzeichnet - steht die Entscheidung, die dem Modell den Namen verleiht. Die „Entscheidung“ entspricht den zuvor für die Trainingsdaten definierten Labels.

Bei dem Einsatz klassischer Entscheidungsbäume besteht jedoch die Gefahr der Überanpassung („Overfitting“"59): Ein zu lange durchgeführtes oder nicht breit genug gestaltetes Training des Modells führt dazu, dass es für die gegebenen Datensätze optimale Ergebnisse liefert, aber für unbekannte Datensätze unbrauchbar ist - eventuell werden sogar irrelevante Merkmale

55 Zurückgehend auf Breiman, Machine Learning 45 Nr. 1 2001, 5.

56 Sammut/Webb, Encyclopedia of Machine Learning and Data Mining.

57 Vgl. zur Erklärung auch Beierle/Kern-Isberner, Wissensbasierte Systeme, S. 107.

58 Vgl. Rokach/Maimon, Data Mining with Decision Trees: Theory and Applications, S. 5.

59 Goodfellow et al., Deep Learning Handbuch, S. 123. 


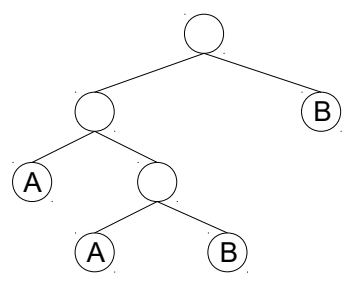

Abbildung 2.1: Entscheidungsbaum, Quelle: eigene Darstellung.

der Datensätze (z. B. die Haarfarbe der Passagiere) in die Überlebenschancen miteinbezogen.

Random Forest-Modelle sollen dem entgegenwirken, indem nicht nur ein einzelner Baum zum Einsatz kommt, sondern eine vom Entwickler bestimmbare Vielzahl (daher „Forest“ - „Wald“) ${ }^{60}$ vgl. Abbildung 2.2. Außerdem wird neben der „Bagging-Technik“ ${ }^{\text {661 }}$ nur eine zufällig gewählte Teilmenge der Features in die Bäume einbezogen, wodurch eine Menge unterschiedlicher Bäume entsteht. Letztendlich durchläuft dadurch jeder Datensatz verschiedene Bäume und wird dementsprechend auf andere Features analysiert. Am Ende jedes Baumes weist das System jedem Datensatz ein Label zu (beispielsweise ,überlebt“" oder ,nicht überlebt“") - auch in Abbildung 2.2 als „A“ (letzte linke Knoten) und „B“ (letzte rechte Knoten) dargestellt. Sodann wird das Ergebnis jedes Baumes für einen Datensatz als Votum gewertet, und am Ende gegenübergestellt, welche Klasse für den Datensatz überwiegt, was letztendlich in der Klassifizierung resultiert. ${ }^{62}$

\section{Künstliche neuronale Netze}

Der Ursprung der „Faszination KI“ lag (nachvollziehbarerweise) unter anderem in dem Wunsch, menschliche Intelligenz nachzubilden. ${ }^{63}$ Da liegt es nahe, dass einige Ansätze der KI-Technologien sich zumindest teilweise an den grundlegenden Strukturen des menschlichen Gehirns orientieren.

60 Breiman, Machine Learning 45 Nr. 1 2001, 5, 6.

61 Bagging, kurz für „Bootstrap Aggregating“, beschreibt eine Datensatzauswahlmethode, bei der aus der Gesamtmenge der Datensätze Teilmengen gebildet werden, die aber Duplikate enthalten dürfen, Ders., Machine Learning 45 Nr. 1 2001, 5, 5; Goodfellow et al., Deep Learning Handbuch, S. 285.

62 Breiman, Machine Learning 45 Nr. 1 2001, S. 7.

63 Vgl. § 2 A.. 

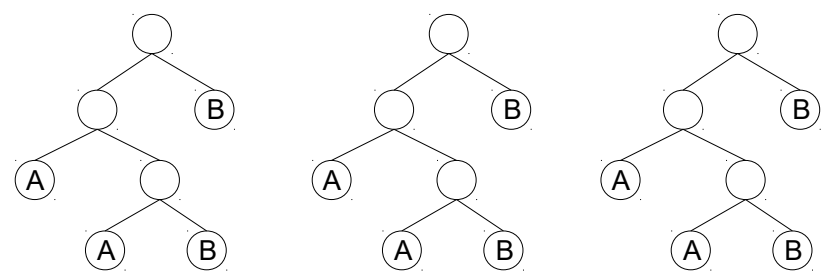

Abbildung 2.2: Random Forest, Quelle: eigene Darstellung.

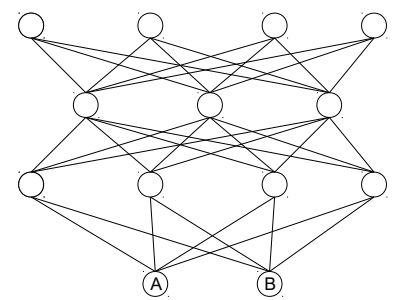

Abbildung 2.3: Künstliches neuronales Netz mit vier Schichten, Quelle: eigene Darstellung.

Wir meinen heute zu wissen, dass das Gehirn im wesentlichen - einfach gesagt - ein Netzwerk spezialisierter Neuronen (Nervenzellen) ist, die durch Synapsen miteinander verbunden sind. ${ }^{64}$ Dabei kann jede Nervenzelle durch mehrere Synapsen mit anderen Neuronen kommunizieren. ${ }^{65}$ Neuronen werden durch Energie aktiviert, und der aktivierende elektrische Impuls wird mittels chemischer Überträgersubstanzen weitergeleitet. ${ }^{66}$ Verbindungen, die viel genutzt werden, werden verstärkt. ${ }^{67}$

Sogenannte KNNs, insbesondere in der Variante tiefer Neuronaler Netze (Deep Learning) sind zu unterschiedlichen Graden von der (vermuteten)

64 Liebmann/Gertz, Basiswissen Neuroanatomie, S. 1.

65 Dies., Basiswissen Neuroanatomie, S. 3; Huggenberger et al., Neuroanatomie des Menschen, S. 6.

66 Liebmann/Gertz, Basiswissen Neuroanatomie, S. 1.

67 Rahmann/Rahmann, Das Gedächtnis: Neurobiologische Grundlagen, S. 224. 
Struktur des menschlichen Gehirns inspiriert. ${ }^{68}$ Mit ihnen wird nicht etwa wie der Name vermuten lässt - direkt versucht, Gehirnfunktionen nachzubauen, sondern vielmehr werden diese Modelle eingesetzt um Annäherungen an statistische Funktionen zu erreichen und dadurch statistische Generalisierungen zu erzielen ${ }^{69}$ (auch dies ist für den mathematischen Laien als Mustererkennung vorstellbar).

In einer visuellen Darstellung ähneln die KNNs auf den ersten Blick einer Baumstruktur. ${ }^{70}$ Der auffälligste Unterschied ist, dass ein Knoten (Neuron) mehrere Vorgänger haben kann. Größere Unterschiede sind erst auf den nächsten Blick - im Folgenden anhand des Titanic-Beispiels - zu erkennen.

Ein einfaches KNN untersucht einen Datensatz in der ersten Schicht (,Input Layer") nach verschiedenen Kriterien (im hiesigen Beispiel etwa die Eigenschaften der Titanic-Passagiere). Die erste Schicht hat also so viele Input-Neuronen wie Eigenschaften untersucht werden sollen. ${ }^{71}$ Die letzte Schicht („Output Layer“) liefert das Ergebnis (Überlebenswahrscheinlichkeit - in Abbildung 2.3 markiert als „A“ und „B“). Die hauptsächliche Arbeit wird im Rahmen des Deep Learnings in den dazwischenliegenden „Hidden Layers ${ }^{\text {“72 }}$ verrichtet. In diesen werden - je nach Art des Netzwerkes mithilfe unterschiedlichster mathematischer Funktionen - Feinjustierungen vorgenommen oder verschiedene Aspekte des Inputs betrachtet. In unserem Beispiel könnten etwa die Neuronen der Hidden Layers analysieren, ob bestimmte Kombinationen von Eigenschaften vorliegen (zum Beispiel Alter $>12$ und oberste Reiseklasse in einem Neuron, Alter $<12$ und Anzahl Familienmitglieder $<4$ in einem weiteren, etc.) und dementsprechend

68 Kaplan, Artificial intelligence: What everyone needs to know, S. 28; Goodfellow et al. weisen darauf hin, dass nicht alle Deep Learning-Forscher sich von den Neurowissenschaften inspirieren lassen, für den eigentlichen Nachbau von Gehirnfunktionen wird auf das Gebiet der Computational Neuroscience verwiesen, Goodfellow et al., Deep Learning Handbuch, S. 18, von dieser Inspiration rührt auch die Bezeichnung als künstliches ,neuronales“ Netz her, Dies., Deep Learning Handbuch, S. 186.

69 Dies., Deep Learning Handbuch, S. 186.

70 Hier zur Verdeutlichung der Ähnlichkeit ebenfalls vertikal dargestellt - in der Regel erfolgt die Abbildung künstlicher neuronaler Netze in der Horizontalen.

71 Nielsen, Neural Networks and Deep Learning, Kap. 1, The Architecture of Neural Networks.

72 Der Name suggeriert, dass hier etwas im Versteckten passiert. Dies ist irreführend, denn „Hidden“ weist lediglich darauf hin, dass keine Schnittstelle - z. B. zum Nutzer - besteht, bzw. dass es sich weder um die Input- noch um die Output-Schicht handelt, vgl. auch Ders., Neural Networks and Deep Learning, Kap. 1, The Architecture of Neural Networks. 
Wahrscheinlichkeitswerte anpassen, welche dann wiederum gewichtet weitergeleitet werden (hier wieder die Parallele zu den energieweiterleitenden Synapsen im Gehirn). Bei künstlichen neuronalen Netzen wird die Struktur vor dem Training festgelegt, das Netz ,wächst“ also im Unterschied zu Entscheidungsbäumen nicht. Variabel sind nur die sogenannten Gewichte (,Weights“) und Verzerrungsparameter ${ }^{73}$ („Biases“). Diese Werte werden im Rahmen des Trainingsvorgangs verändert und optimiert.

Dies ist eine stark vereinfachte Darstellung der möglichen Funktionsweise solcher Modelle. Für das Verständnis der Arbeit ist eine tiefergehende Einsicht in die verwendeten statistischen Funktionen etc. nicht erforderlich und wird dem Leser an dieser Stelle erspart. ${ }^{74}$ Wichtig festzustellen ist jedoch, dass die Art und die „Architektur“ des Modells einen wesentlichen Einfluss auf die Einsatztauglichkeit und Genauigkeit des Modells haben. Der Entwickler steht also stets vor der schwierigen Aufgabe, die richtige Anzahl Schichten, Neuronen je Schicht, Rückkopplungen, Aktivierungsfunktionen und dergleichen auszuwählen. ${ }^{75}$

\section{Sonderfall: Generative Adversarial Networks}

Während in den bisherigen Ausführungen Modelle im Vordergrund standen, die Daten analysieren und Werte ausgeben, erfolgt nun die Einführung einer sich davon doch wesentlich unterscheidenden Art: Sogenannte generative Modelle, bzw. insbesondere generative adversariale Netzwerke (GANs). ${ }^{76}$ Diese Sonderform in Gestalt einer Kombination von KNNs zielt nicht darauf ab, einen Wert zu schätzen, sondern vielmehr darauf, Daten - in Form von Bildern, Texten, Musik etc. - zu erzeugen. So komplex die Ergebnisse erscheinen mögen, so simpel ist die Idee dahinter - hier erklärt anhand der Erzeugung von Bildern, auf denen Menschen zu erkennen sind:

73 Goodfellow et al., Deep Learning Handbuch, S. 121: „Diese Bezeichnung leitet sich aus der Sichtweise ab, dass ohne jegliche Eingabe die Ausgabe der Transformation zu [dem Achsenschnittpunkt] $b$ verschoben ist. Der Begriff darf nicht mit einer statistischen Verzerrung [...] verwechselt werden.“.

74 Stattdessen wird verwiesen auf Dies., Deep Learning Handbuch, Kaplan, Artificial intelligence: What everyone needs to know und Nielsen, Neural Networks and Deep Learning.

75 Ders., Neural Networks and Deep Learning, Kap. 1, The architecture of neural networks.

76 Eingeführt 2014 von Goodfellow et al., Generative Adversarial Nets. 
Zwei künstliche neuronale Netze werden miteinander verbunden - ein generativer und ein adversarialer (gegnerischer) Part. Der adversariale Part ist ein klassifizierendes Modell, das schon vortrainiert wurde mit Bildern von Menschen, und das der im vorigen Abschnitt beschriebenen Funktionsweise entspricht. Der generierende Part wurde nicht vortrainiert, sondern bekommt vom Entwickler lediglich Vorgaben wie zum Beispiel die Größe des zu erzeugenden Bildes. Sodann erzeugt das generierende Modell zunächst quasi zufälliges „Rauschen“, setzt also beliebige Pixel auf beliebige Werte. Das Ergebnis wird dann zum Input des gegnerischen Parts. Dieser hat nun die alleinige Aufgabe, zu unterscheiden, ob der Input aus den ihm bekannten, „echten“ Bildern stammt oder durch das generierende Modell erzeugt wurde - muss also unterscheiden zwischen „Original“ und „Fake“ ${ }^{77}$ Diese zwei Kategorien sind die Klassen, die dem gegnerischen Part vorgegeben sind und stellen die Trainingsaufgabe für den gegnerischen Part dar. Dem generierenden Part wird zurückgemeldet, ob der gegnerische Part das Fake als ein solches erkannt hat (bzw. mit welcher Wahrscheinlichkeit es für ein solches gehalten wurde), und startet dann den nächsten Versuch, indem einige Pixelwerte angepasst werden, bis die Wahrscheinlichkeit sinkt, dass es sich um ein Fake handelt. Das generierende Modell versucht also, das gegnerische Modell ,auszutricksen“, indem es seine Erzeugnisse so optimiert, dass das gegnerische Modell Fake und Original nicht mehr unterscheiden kann. ${ }^{78}$ Gleichzeitig versucht das gegnerische Modell, seine Fähigkeit, zwischen Original und Fake zu differenzieren, zu optimieren. Auf diese Weise entstehen eindrucksvolle Ergebnisse: Einige Beispiele, die auf diese Weise entstanden sind, sind das 2018 versteigerte „Gemälde“ des französischen Künstlerkollektivs Obvious „Edmond de Belamy“79 sowie das Projekt „Next Rembrandt“" 80 in dessen Rahmen der adversariale Part mit Rembrandt-Werken trainiert wurde und der generative Part die Aufgabe bekam, Werke zu generieren, die der adversariale Part für ,einen echten Rembrandt“ halten würde.

Da hier zumindest der Art der Ergebnisse der GANs nach potenziell „Werke" entstehen können, lohnt es sich, generative Modelle und insbesondere den Entstehungsprozess der Erzeugnisse genauer zu betrachten. Damit wird sich der vierte Teil dieser Arbeit befassen, weitere Beispiele werden in $\S 10 \mathrm{C}$. untersucht.

77 Goodfellow et al., Generative Adversarial Nets, S. 1.

78 Dies., Generative Adversarial Nets, S. 2.

79 Vgl. https://obvious-art.com/portfolio/edmond-de-belamy/ (Stand: 22.02.2021); vgl. zur Erklärung außerdem $\S 10$ C.III..

$80 \mathrm{Vgl}$. https://nextrembrandt.com (Stand: 22.02.2021). 


\section{Einige grundlegende Begriffe}

Es folgt eine Erläuterung einiger grundlegender Begriffe, die teilweise schon zur Erklärung des Machine Learnings herangezogen werden mussten. Dort waren sie für das Verständnis der wesentlichen Konzepte nicht zwingend erforderlich, andererseits ist für das Verständnis der Begriffe eine Vorstellung von Machine Learning förderlich, weshalb diese Reihenfolge gewählt wurde. Es handelt sich um eine Auswahl von Begriffen aus dem Kontext des maschinellen Lernens, die im Rahmen dieser Arbeit immer wieder zur Verwendung kommen. Auf umfangreiche Ausführungen wird allerdings verzichtet, es erfolgt eine Beschränkung auf die für das Kontextverständnis hinreichenden Informationen.

\section{Klassifikation, Klassifizierung}

Die Begriffe Klassifikation und Klassifizierung werden in der deutschsprachigen Machine Learning-Literatur weitgehend synonym verwendet. Beides bezeichnet laut Duden einen Vorgang der Einordnung in eine Klasse. ${ }^{81} \mathrm{Im}$ Kontext des Machine Learnings sind klassifizierende Modelle einfach gesprochen Systeme, die einem gegebenen Eingangswert einen Ausgangswert aus einem vorgegebenen Katalog zuweisen. ${ }^{82}$ Zur Verdeutlichung ein Beispiel: Dem System wird ein Bild zugeführt, auf dem ein Haus und ein Baum abgebildet sind. Wenn das System gelernt hat, (unter anderem) Häuser und Bäume zu erkennen, wird es die Klassen „Haus“ und „Baum“ ausgeben.

81 Vgl. Duden „Klassifikation“, https://www.duden.de/rechtschreibung/Klassifikation (Stand: 22.02.2021) sowie Duden „Klassifizierung“, der Eintrag verweist für die Wortbedeutung auf die Klassifikation und lässt vermuten, dass beide Worte synonym verstanden werden können, https://www.duden.de/rechtschreibung/Klassifizierung (Stand: 22.02.2021), wenngleich z. B. Wikipedia darauf hinweist, dass Klassifikation eigentlich als Ergebnis der Klassifizierung zu verstehen sei, https://de.wikipedia.org/ wiki/Klassifizierung\#Begriffsabgrenzung (Stand: 22.02.2021).

82 Goodfellow et al., Deep Learning Handbuch, S. 109 mit weiteren Beispielen. 


\section{Regression}

Regression (bzw. eigentlich präziser Regressionsanalyse) hingegen beschreibt einen Vorgang der Annäherung an einen Wert. ${ }^{83}$ Das System versucht, eine Frage, die die Angabe eines Wertes erwartet, durch Annäherung an im Training Gelerntes zu beantworten. ${ }^{84}$ Beispiel: Dem System wird ein Bild zugeführt, auf dem ein Mensch abgebildet ist. Wenn das System gelernt hat, das Aussehen von Menschen und Alterswerte in Relation zu setzen, wird es ein geschätztes Alter ausgeben. Die Regressionsanalyse ist ein statistisches Verfahren, auf dessen tiefergehende, aber hier für den weiteren Verlauf unnötige Erklärung an dieser Stelle verzichtet wird.

Goodfellow et al. erläutern neben Klassifizierung und Regression noch einige weitere klassische ML-Aufgaben - darunter auch die Umwandlung unstrukturierter Daten in Text (Bsp: Text-to-Speech-Umwandlung), maschinelle Übersetzungen, Anomalieerkennungen, Sprachsynthese (erlaubt es etwa digitalen Assistenten zu ,sprechen“, ohne dass vorher ganze Wörter oder Phrasen vom Menschen eingesprochen wurden) u. v. m.. ${ }^{85}$

\section{3. Überwachtes Lernen (Supervised Learning)}

Überwachtes Lernen wird die Lernmethode genannt, wenn die Daten, mit denen das System lernt, bereits mit Labels (Klassen) versehen wurden. Auf das obige Beispiel der Klassifikation übertragen: Wenn das System die Fähigkeit, das Haus und den Baum zu erkennen, dadurch gelernt hat, dass ihm zuvor eindeutig als Haus und Baum bezeichnete Bilder im Rahmen des Lernprozesses ,,vorgeführt“ wurden, wird von überwachtem Lernen gesprochen. Analoges gilt für ein System, das mit Regression arbeitet. ${ }^{86}$

83 Hinweis: hier ist der statistische Wortgebrauch relevant, es handelt sich nicht um Regression im wirtschaftlichen Sinne, vgl. auch Duden „Regression“, https://www. duden.de/rechtschreibung/Regression (Stand: 22.02.2021).

84 Goodfellow et al., Deep Learning Handbuch, S. 109.

85 Dies., Deep Learning Handbuch, S. 109 ff..

86 Vgl. auch Ertel, Grundkurs Künstliche Intelligenz: Eine praxisorientierte Einführung, S. 313; Goodfellow et al., Deep Learning Handbuch, S. 115. 
4. Unüberwachtes Lernen (Unsupervised Learning)

Unüberwachtes Lernen hingegen überlässt dem Algorithmus das Auffinden und Zuweisen von Gemeinsamkeiten, bzw. Klassen. Die Aufgabe könnte heißen „Finde Gemeinsamkeiten auf diesen Bildern!“ ${ }^{87}$

\section{Lernen durch Verstärkung (Reinforcement Learning)}

Reinforcement Learning beschreibt einen Prozess, der ohne Trainingsdaten auskommt. Hierbei könnte sich das System zum Beispiel durch Ausprobieren der Lösung nähern, wobei zuvor programmatisch „Belohnungen“ und „Bestrafungen“ für gute respektive schlechte oder aufwendige Lösungen vorgesehen sind. Das System versucht, während es den Weg zur Lösung sucht, die Belohnungen zu maximieren. ${ }^{88}$ Der Input kann sich etwa aus der Aufzeichnung von Umgebungsdaten durch Sensoren ergeben. ${ }^{89}$

6. Parameter, Gewichte, Biases, Hyperparameter, Topologie, Schichten, Netzstruktur, Architektur

Die in der Überschrift genannten Begriffe lassen sich am besten gemeinsam erklären. Sie sind grundsätzlich zu teilen in zwei Gruppen:

- Parameter, Gewichte, Biases

- Hyperparameter, Topologie, Schichten, Netzstruktur, Architektur.

Die erste Gruppe beschreibt den Teil der ML-Modelle, der im Rahmen des Trainings automatisch angepasst und optimiert wird. Gewichte, bzw. engl. Weights, und Biases sind Begriffe, die insbesondere im Kontext von KNNs relevant sind. Dort beschreiben sie die veränderlichen Werte, die einfach gesagt - den Anteil eines Inputs am Ziel-Output ausmachen. Für eine ausführlichere Beschreibung vgl. § 2 B.II.2.. Der allgemeinere (und in dieser

87 Ausführlich zu Unsupervised Learning: Hinton/Sejnowski, Unsupervised Learning; Goodfellow et al., Deep Learning Handbuch, S. 115.

88 Vgl. auch Ertel, Grundkurs Künstliche Intelligenz: Eine praxisorientierte Einführung, S. 313.

89 Vgl. auch Goodfellow et al., Deep Learning Handbuch, S. 116 f. mit weiteren Nachweisen zu Reinforcement Learning. 
Arbeit überwiegend verwendete) Begriff ist der der Parameter. Dieser ist auch für andere ML-Modelle zutreffend und erfasst etwa für Random ForestModelle, die mit Python erzeugt werden, die variablen Split-Schwellenwerte.

Die Begriffe der zweiten Gruppe haben gemeinsam, dass sie im Rahmen des Trainings nicht automatisiert angepasst und zudem nicht innerhalb des eigentlichen Algorithmus eingestellt werden.${ }^{90}$ Topologie, Netzstruktur und Architektur können synonym verwendet werden und beschreiben unter anderem, wie viele Schichten ein Modell hat und wie diese aufgebaut sind. Schichten (engl. Layer) sind - im Falle eines KNN - Neuronen, die parallel abgearbeitet werden (vgl. dazu Abbildung 2.3: das abgebildete KNN besteht aus 13 Neuronen, die auf 4 Schichten verteilt sind).

$\mathrm{Zu}$ den Hyperparametern gehören alle Angaben und Einstellungen, die für ein Modell vorgenommen werden - für ein KNN etwa die Anzahl der Schichten und der Neuronen je Schicht, für einen Random Forest u. a. die maximale Tiefe des Baumes. Veränderungen an den Hyperparametern sind stets Gestaltungsentscheidungen des Entwicklers, um die Modellperformanz zu optimieren. Weil sie durch die Einstellungen das Modell, würde man es visuell abbilden, im Aufbau verändern, werden die Hyperparameter auch als Netzstruktur (speziell für KNN), Architektur des Modells oder Topologie bezeichnet, obwohl diese Begriffe darüber hinwegtäuschen können, dass eine Sammlung von Hyperparametern mitunter auch zum Beispiel die von den Neuronen zu verwendenden Funktionen enthält. Diese Arbeit verwendet den Begriff der Hyperparameter. Falls einmal die Begriffe Struktur, Architektur oder Topologie genannt werden, so sind damit immer die Hyperparameter gemeint.

7. Trainingsprozess, Trainingsdaten, Trainingsergebnisse

Auch wenn der Trainingsprozess sich im Detail für verschiedene Modelle und Aufgaben unterscheiden kann, ${ }^{91}$ erfolgt hier eine verallgemeinerte Beschreibung für klassifizierende künstliche neuronale Netze, um einen Überblick zu verschaffen. Damit die beschriebenen Modelle tatsächlich Ergebnisse liefern, werden diese mit Trainingsdaten auf ihre zugrundeliegende Aufgabe trainiert. Trainingsdaten sind zu analysierende Daten (Bilder, Texte, Zahlen, ...), die für den überwachten Lernprozess - bereits den korrekten Kategorien zugeord-

90 Goodfellow et al., Deep Learning Handbuch, S. 107.

91 Dies., Deep Learning Handbuch, S. 114. 
net sind (,gelabelt sind“) ${ }^{92}$ Die Zuordnung kann beispielsweise geschehen, indem die die Daten enthaltenden Dateien entsprechend benannt werden oder indem die Dateien in den Labels entsprechenden Ordnern abgelegt werden. ${ }^{93}$ Üblicherweise separiert der Entwickler von den Trainingsdaten noch einige Test- bzw. Validierungsdaten, um das fertig trainierte Modell mit Daten testen zu können, die demselben nicht bekannt sind.

Für das Training wird im überwachten Lernen versucht, einen Zusammenhang zwischen Input (Trainingsdaten) und Output (Label) zu finden. ${ }^{94}$ Für künstliche neuronale Netze ist in der Regel vorgegeben, welche Eigenschaften der Trainingsdaten analysiert werden sollen. Dabei durchläuft jeder Trainingsdatenpunkt das Modell einzeln. In jedem Neuron wird ein unterschiedlicher Aspekt des Inputs untersucht (bei Bildern z. B. die einzelnen Pixel - die erste Schicht hätte dann so viele Inputneuronen wie das Bild Pixel hat, evtl. noch erweitert um die Farbwerte ${ }^{95}$ ). Um zu messen, wie gut das Modell schon gelernt hat, wird eine Zielfunktion definiert. ${ }^{96}$ Wenn es sich dabei um eine Fehlerquotenfunktion handelt, gilt: je niedriger der Fehler (also die Abweichung des Ergebnisses von dem Ergebnis, das durch das Label vorgegeben ist ${ }^{97}$ ), desto besser findet die Formel (das Modell) zu dem Ergebnis, das dem Label entspricht. ${ }^{98}$ Wenn der Fehler nahe Null ist, findet das Modell für alle Trainingsdaten den richtigen Output, und hat dementsprechend gute Gewichte und Biases gewählt.

Gut ist das Modell, wenn es auch für Daten, die nicht in den Trainingsdaten enthalten waren - sogenannte Testdaten - mit möglichst geringer Fehlerquote die richtigen Ausgaben erzeugt. ${ }^{99}$ Dazu wird eine (von der Zielfunktion verschiedene) Funktion konzipiert, die die Gewichte der Neuronen so optimiert,

92 Goodfellow et al., Deep Learning Handbuch, S. 115; im Vorgang des unüberwachten Lernens sind keine Labels erforderlich, da aus den Daten Zusammenhänge erkannt werden sollen, die auch dem Datensammler noch unbekannt sind.

93 Vgl. z. B. die Ausführungen in https://www.tensorflow.org/tutorials/load_data/images (Stand: 22.02.2021).

94 Vgl. Goodfellow et al., Deep Learning Handbuch, S. 154 f..

95 Dies., Deep Learning Handbuch, S. 109.

96 Dies., Deep Learning Handbuch, S. 113 f..

97 Der geneigte Schuldrechtler denkt hier „Abweichung der Ist-Beschaffenheit von der Soll-Beschaffenheit“...

98 Es gibt verschiedene Möglichkeiten, die Performanz eines Modells zu messen, die auch von der Art des Modells abhängt - die Kostenfunktion für Klassifizierungsaufgaben (auch Mean Squared Error, Fehlerquote genannt) ist eine davon, vgl. auch Nielsen, Neural Networks and Deep Learning, Kap. 1, Learning with gradient descent und Goodfellow et al., Deep Learning Handbuch, S. 114, 119.

99 Dies., Deep Learning Handbuch, S. 114. 
dass die Fehlerquote sinkt, ${ }^{100}$ ohne dabei aber die Trainingsdaten auswendig zu lernen: Denn das Modell soll ja auch unbekannte Daten erfolgreich verarbeiten. Um das sicherzustellen, werden die Testdaten eingesetzt.

Trainingsergebnisse sind die errechneten bzw. optimierten Parameter. Die Unterscheidung zwischen Trainingsdaten und Trainingsergebnissen ist erkennbar wichtig, wird jedoch nicht an allen Stellen konsequent durchgehalten. ${ }^{101}$ Diese Arbeit verwendet die Begriffe so, wie sie hier definiert wurden.

\section{Technische Einordnung}

Häufig besteht Unklarheit darüber, was „KI“ bzw. ein „ML-Modell“ eigentlich ist, insbesondere welcher technische „Charakter" hier zugrunde liegt. Handelt es sich um eine Datei, um ein Programm, oder um etwas ganz anderes? Hierauf wird in $\S 6$ vertieft eingegangen, weil es dort besonders relevant wird. An dieser Stelle sei nur so viel gesagt: Ein ML-Modell funktioniert nicht ohne Computerprogrammcode, das Herzstück (oder „Hirnstück“?) besteht jedoch in der Regel in einer Vielzahl von Werten, die in Dateiform losgelöst vom Computerprogramm - vorliegen können. Und „das Herzstück“ ist letztendlich, Werte eingesetzt und ausgeschrieben, eine enorm lange mathematische Formel mit sehr vielen Parametern. Ein ML-System lässt sich zerlegen in vier Kernkomponenten: einen Datensatz, der analysiert werden soll, ein Modell, das der Datensatz durchläuft, eine mathematische Kostenfunktion, die die Performanz misst, und einen Optimierungsalgorithmus, der das Modell verändert. ${ }^{102}$ Diese Komponenten sind in der Implementierung (also jenseits der statistisch-mathematischen Theorie) verbunden durch Computerprogrammcode. Dabei ist es irrelevant, ob es sich um ein Random Forest-Modell oder ein künstliches neuronales Netz handelt.

100 Goodfellow et al., Deep Learning Handbuch, S. 119.

101 Vgl. z. B. Kaulartz/Braegelmann, Rechtshandbuch Artificial Intelligence, Kap. 7.1 Rn. 49 ff., dort ist die Rede von „Trainingsdaten“, gemeint sind jedoch vermutlich „Trainingsergebnisse“.

102 Goodfellow et al., Deep Learning Handbuch, S. 108. 


\section{Verhältnis des Machine Learning zu Text und Data Mining}

Der Begriff des Text und Data Mining (TDM) taucht bereits heute sowohl in EU-Richtlinien (vgl. z. B. Art. 3 DSM-RL) als auch im deutschen Urheberrechtsgesetz auf (vgl. § 60d UrhG), und fällt sogleich auch immer wieder im Kontext des maschinellen Lernens. An dieser Stelle werden die beiden Begriffe für ein besseres Verständnis ins Verhältnis gesetzt.

Aus der BT-Drs. 18/12329 ergibt sich, dass der deutsche Gesetzgeber unter Text und Data Mining die Auswertung einer „Vielzahl von Texten, Daten, Bildern und sonstigen Materialien“ versteht, ,um so neue Erkenntnisse zu gewinnen." ${ }^{103}$

Grundsätzlich ist zu unterscheiden zwischen Text Mining und Data Mining: Ersteres analysiert unstrukturierte Textdaten, letzteres befasst sich mit strukturierten Daten, z. B. aus Datenbanken. ${ }^{104}$ Dabei wird Machine Learning insbesondere für die Zwecke des Data Minings eingesetz ${ }^{105}$ Machine Learning fokussiert auf die Vorhersage bzw. Wahrscheinlichkeitsschätzung unbekannter Werte, während TDM sich in erster Linie mit bekannten Daten befasst und diese auf Muster, Strukturen und Zusammenhänge analysiert. Teilweise werden TDM und ML synonym verwendet, ${ }^{106}$ oder nur das unüberwachte Lernen dem Data Mining gleichgesetzt ${ }^{107}$. Sinnvoll erscheint es, zumindest den Lernprozess des Machine Learnings, der mit bestehenden, sicheren Daten arbeitet, als (Text und) Data Mining-Verfahren zu betrachten. Dafür spricht, dass die Aufgabe - Muster in den Daten zu erkennen - die gleiche ist, auch wenn Machine Learning später noch einen Schritt weiter geht und daraus Vorhersagen für unbekannte Daten ableiten will oder gar ganz neue Daten erzeugt. Dies erleichtert zumindest auch die urheberrechtliche Bewertung des Lernprozesses, und ermöglicht es, die für das TDM bestehenden (und entstehenden) Regelungen auch auf den Lernprozess des ML anzuwenden. Falsch wäre es jedoch, TDM und ML grundsätzlich immer synonym zu verwenden, dafür sind die Begriffe nicht trennscharf genug definiert.

103 BT-Drs. 18/12329 S. 22.

104 Klass, Wirtschaftsinformatik \& Management 11 Nr. 4 2019, S. 267.

105 Ders., Wirtschaftsinformatik \& Management 11 Nr. 4 2019, S. 267.

106 Vgl. z. B. Faul, A Concise Introduction to Machine Learning., S. XVII.

107 Bell, Machine Learning, S. 4. 
2. Verhältnis des Machine Learning zu Expertensystemen

Ein naheliegender Versuch, den menschlichen Verstand nachzubilden, liegt darin, einer Maschine möglichst viel Wissen zu vermitteln, und die Maschine aus der ihr zur Verfügung stehenden Wissensbasis Antworten geben zu lassen. Solche „Expertensysteme“ bzw. auch allgemeiner ,wissensbasierte Systeme“ ${ }^{\text {108 }}$ genannten Ansätze werden seit 1974 entwickelt. ${ }^{109}$ Beierle fasst bestehende Definitionen von Expertensystemen wie folgt zusammen:

„Ein Expertensystem ist ein Computersystem (Hardware und Software), das in einem gegebenen Spezialisierungsbereich menschliche Experten in Bezug auf ihr Wissen und ihre Schlussfolgerungsfähigkeit nachbildet. " ${ }^{110}$

Zusammengesetzt sind solche Systeme grundsätzlich aus einer Wissensbasis und einer wissensverarbeitenden Komponente, ${ }^{111}$ hinzu kommen eine Wissenserwerbs- und eine Erklärungskomponente sowie eine Dialogkomponente. ${ }^{112}$ Sowohl Verfahren maschineller Lernsysteme als auch allgemein Data Mining können grundsätzlich dazu eingesetzt werden, Expertensysteme zu implementieren, ${ }^{113}$ so ist es etwa möglich, die das Expertenwissen repräsentierenden Regeln deduktiv aus Daten zu lernen. Es ist jedoch auch hier zu vermeiden, die Begriffe des maschinellen Lernens und der Expertensysteme, oder auch Expertensysteme und KI, synonym zu verwenden.

\section{Zusammenfassung}

Festzustellen ist, dass Modelle maschinellen Lernens keine gänzlich undurchsichtige mystischen Gebilde sind, sondern vielmehr Software bzw. Algorithmen, die auf mathematischen bzw. statistischen Funktionen basieren und eine große Menge an Daten benötigen, um im Einsatz Ergebnisse in Form von Wahrscheinlichkeitswerten liefern zu können.

108 Puppe, XPS-99, Vorwort; zur Unterscheidung vgl. Beierle/Kern-Isberner, Wissensbasierte Systeme, S. 11.

109 McCorduck, Machines Who Think, S. XXVIII identifiziert das Programm MYCIN von T. Shortliffe als erstes „Expertensystem“.

110 Beierle/Kern-Isberner, Wissensbasierte Systeme, S. 12.

111 Dies., Wissensbasierte Systeme, S. 17.

112 Dies., Wissensbasierte Systeme, S. 18.

113 Vgl. für einige Beispiele Dies., Wissensbasierte Systeme, S. 99 ff.; Looney, Expert Systems With Applications 6 1993, 129 ff.. 


\section{Zweiter Teil \\ Die Maschine lernt - auch anhand urheberrechtlich geschützter Daten?}

Das auf viele urheberrechtliche Sachverhalte zutreffende Gleichnis ,Zwerge auf den Schultern von Riesen“ 114 beschreibt Modelle maschinellen Lernens ziemlich genau. Ohne Trainingsdaten ist selbst das beste (trainingsdatenbasierte) Modell nicht zu verwenden, ${ }^{115}$ und mit der Qualität und Quantität dieser Daten steigen und fallen die Qualität der Ergebnisse sowie die Nutzbarkeit des Modells. Auf das Gleichnis übertragen sind die Modelle vergleichsweise kleine Gebilde - Zwerge - die auf monumentalen Datensammlungen - Riesen - aufsetzen. Aus dieser Perspektive wird die Relevanz der Trainingsdaten besonders deutlich.

Im Zuge dessen stellen sich die Fragen, was im Trainingsprozess eigentlich mit den Trainingsdaten passiert: Werden etwa urheberrechtlich relevante Handlungen vorgenommen? Inwiefern könnten die Trainingsdaten im fertigen Modell enthalten sein? Liegt eine Bearbeitung oder eine freie Benutzung vor, oder findet das Urheberrecht hier gar keine Anwendung? Wie stehen die Text- und Data-Mining-Schranken § 60d UrhG bzw. § 44b UrhG-E zu maschinellen Lernprozessen?

Von der Diskussion in dieser Arbeit ausgenommen ist die Frage, wie eine Sammlung von Trainingsdaten selbst geschützt sein kann. Die für alle Textund Data-Mining-Verfahren gleichermaßen vorzunehmenden Handlungen zur Sammlung der Daten bzw. Datenkorpuserstellung im Vorfeld bedürfen keiner gesonderten Betrachtung für Zwecke des Machine Learnings. Hier dürften die allgemeinen Regeln des Datenbankschutzes nach $\S 4$ Abs. 2 UrhG und $\S \S 87 \mathrm{a} f f$. UrhG anwendbar sein, ohne dass sich aus der Natur des Machine Learning-Kontextes Besonderheiten ergeben. ${ }^{116}$

114 Ursprung vermutlich in einem Brief von Peter von Blois an Reginald, Bishop of Bath, Epistolae 92 (1180), zitiert in Merton, On The Shoulders Of Giants, S. 216 f..

115 Es sei denn, es handelt sich um ein trainingsdatenloses selbstlernendes Modell, das allein anhand von Regeln und Reinforcement lernt, wie z. B. AlphaGo, vgl. https:// deepmind.com/research/case-studies/alphago-the-story-so-far (Stand: 22.02.2021), das als Input lediglich das Go-Feld bekommt.

116 Den Schutz als Datenbankwerk gem. § 4 Abs. 2 UrhG eher ablehnend, für eine Anwendung von $\S \S 87 \mathrm{a}$ ff. UrhG und auch weitere Schutzmöglichkeiten diskutierend Hacker, GRUR 2020, 1025, 1028. 


\section{§ 3 Urheberrechtlich relevante Vorgänge im maschinellen Lernprozess}

Zunächst erfolgt eine Analyse der technischen Vorgänge des Einlesens und des Trainings anhand der eingelesenen Daten (Trainingsdaten), um daraus auf eventuell urheberrechtlich relevante Vorgänge zu schlussfolgern. Daran schließt sich eine Darstellung der geltenden und der im Rahmen der Umsetzung der DSM-Richtlinie zu erwartenden Schranken an, um eine Einordnung aus der Perspektive unterschiedlicher Nutzergruppen vornehmen zu können.

\section{A. Technische Beleuchtung des maschinellen Lernprozesses}

Um die eingangs gestellten Fragen zu beantworten, erscheint es dienlich, den ablaufenden Prozess genauer unter die Lupe zu nehmen und dabei den Fokus auf die Trainingsdaten zu legen. Dabei ist freilich eine Betrachtungsweise zu finden, die so abstrakt ist, dass sie auf eine Vielzahl unterschiedlicher ML-Modelle anwendbar ist, und gleichzeitig konkret genug, um die urheberrechtlichen Fragen zufriedenstellend beantworten zu können.

Der Prozess des maschinellen Lernens lässt sich grob in vier Phasen unterteilen, die in Abbildung 3.1 gezeigt sind. Die erste Phase umfasst die Sammlung und Organisation der Daten, die hier nicht weiter thematisiert werden. Wenn die Datensammlung abgeschlossen ist, werden die Daten entweder direkt in das den Trainingsprozess ausführende Programm oder zunächst in ein anderes Programm eingelesen, dass die Daten für den Trainingsprozess anpasst und aufbereitet. Daran schließt sich der eigentliche Trainingsprozess an, mit dem Ziel, ein trainiertes Modell zu produzieren (hier abgebildet sind zwei Möglichkeiten, die auch im weiteren Verlauf diskutiert werden: Modelle in sog. Baumstrukturen und in sog. Netzwerkstrukturen). Wenn das Modell trainiert ist, kann es eingesetzt bzw. für den Einsatz bereitgestellt werden.

Nicht abgebildet, weil für die Fragestellung in diesem Kapitel nicht relevant, ist die Entwicklung des Modells, das die Trainingsdaten später verwendet. Diese Entwicklung kann vor der Trainingsdatensammlung stattfinden (sodass gezielt nach Daten für das Modell gesucht werden kann), oder anhand vorhandener Daten erfolgen und mitunter auch während des Trainingsprozesses iterativ Anpassungen erfordern. 


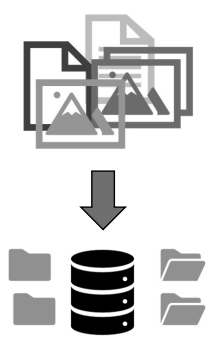

Sammlung \& Organisation der Daten

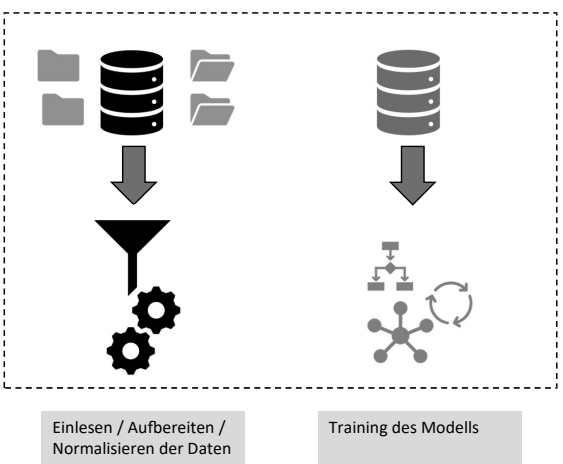

Einsatz / Bereitstellung des fertigen Modells

Abbildung 3.1: Phasen des Machine Learning-Prozesses, eigene Darstellung.

Die folgenden Ausführungen beschränken sich auf die Einlese- und Trainingsvorgänge.

I. Einlesevorgang und Analyse bzw. Aufbereitung der Trainingsdaten

Grundsätzlich wird im Einlesevorgang jeder Datensatz der Datenbasis einzeln auf die gleiche Weise analysiert (und nicht etwa die Trainingsdatensammlung als Ganzes). ${ }^{117}$ Welche Analyseschritte vorgenommen werden, ist davon abhängig, für welche Vorgehensweise der Entwickler sich entscheidet, weshalb diese Vorbereitung bzw. Aufbereitung der Trainingsdaten für jedes MLModell unterschiedlich gestaltet sein kann. Beispiele für Analyseschritte sind das Einlesen eines Bildes in ein von dem Modell verarbeitbares Dateiformat oder ein Zusammenschneiden des Bildes auf eine bestimmte Größe, sowie die Bereinigung der Daten von sogenannten „Ausreißern“, die aufgrund ungewöhnlicher Werte zu Verzerrungen führen könnten. ${ }^{118}$

117 Vgl. z. B. die Vorgehensweise des Einlesens von Trainingsdatenbildern in image_dataset.py ab Zeile 211 bzw. paths_and_labels_to_dataset (...) und path_to_image (...), https://github.com/tensorflow/tensorflow/blob/v2.4.1/tensorflow/python/keras/ preprocessing/image_dataset.py (Stand: 22.02.2021).

118 Osinga, Deep Learning Cookbook, S. 22 f.; Johnstone, What it Means to „Clean“ Data and „Train“ Machine Learning Algorithms. 


\section{Einlesen einzelner oder aller Datensätze}

Die Trainingsdatensammlung kann in unterschiedlichster Form vorliegen: Beispielsweise als eine Vielzahl von Dateien in Ordnern (jede Datei entspricht dann einem Trainingsdatum) oder auch als einzelne Datei (etwa ein Textdokument ${ }^{119}$ oder eine MySQL-Datenbank ${ }^{120}$ ).

Der Unterschied ist aber für die urheberrechtliche Analyse unerheblich, da es letztendlich auf den Zustand vor dem Beginn und nach dem Abschluss des Einlesevorgangs aller Daten ankommen wird.

\section{Umwandlung der Daten in ein maschinenlesbares Format}

Damit ein Machine Learning-Modell mit der Trainingsdatensammlung arbeiten kann, muss es auf diese Daten zugreifen können. Die zu nutzende Datei wird dafür vorübergehend in den Arbeitsspeicher geladen. Für das Training von KNN wird der Input in einen Tensor ${ }^{121}$ oder eine andere Repräsentationsform der Daten ,,̈̈bersetzt“. Die folgenden Ausführungen beschränken sich auf die Vorgehensweise mit Tensoren. Für eine Bilddatei mit einer Dimension von 20 mal 20 Pixeln bedeutet das, dass ein Tensor entsteht, der für jedes Pixel drei Werte (RGB) enthält. ${ }^{122}$ Oder anders gesagt: es entsteht im Arbeitsspeicher ein Konstrukt ähnlich einem Setzkasten mit drei Etagen, wobei jede Etage aus 20 mal 20 Feldern besteht. Die oberste Etage enthält die Rotwerte $(\mathrm{R})$, die mittlere die Grünwerte $(\mathrm{G})$ und die untere die Blauwerte (B). Angenommen, das Pixel in der oberen linken Ecke des Bildes hat den Farbwert „255, 242, 0“123 (gelb, vgl. Abbildung 3.2), dann erhält das Feld der obersten Schicht in der oberen linken Ecke den Wert „255“, das Feld der Grünschicht den Wert ,242“ und und das Feld der Blauschicht den Wert „,0“. Der Machine Learning-Algorithmus arbeitet nur auf der Tensor-Version des Inputs.

119 Dann könnte etwa jedes Wort oder jeder Satz ein Trainingsdatum darstellen.

120 MySQL ist ein Datenbankformat, das bspw. ein Backup einer Datenbank in einer einzigen Datei speichern kann, vgl. z. B. https://dev.mysql.com/doc/refman/8.0/en/ mysqldump.html (Stand: 22.02.2021).

121 Tensoren sind Vektoren mit mehreren Dimensionen, vgl. z. B. Osinga, Deep Learning Cookbook, S. 1.

122 Der Tensor hätte also die Form (20,20,3) - vgl. https://www.tensorflow.org/tutorials/ load_data/images\#visualize_the_data (Stand: 22.02.2021).

123 In der hier gewählten Schreibweise ist Schwarz definiert als 0, 0, 0 und Weiß als 255, 255, 255. 

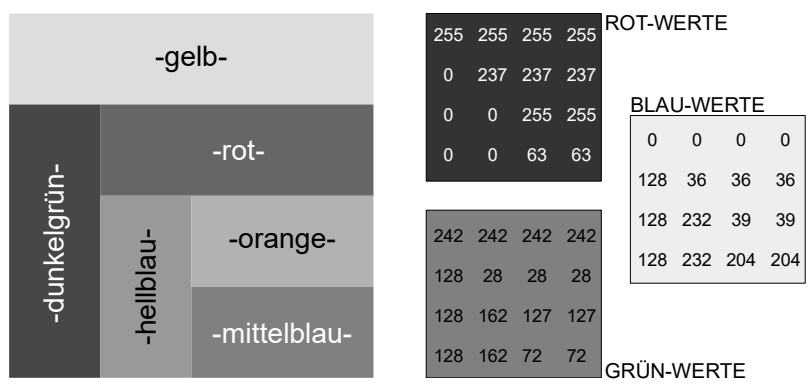

Abbildung 3.2: Bild mit 4x4 Pixel, eigene, stark vergrößerte Darstellung.

Abbildung 3.2 veranschaulicht die Umwandlung der Werte. Links ist ein Ausgangsbild - also ein Beispiel für mögliche Trainingsdaten - abgebildet, ${ }^{124}$ rechts die Farbwerte in den „Setzkästen“, die ausgelesen werden. Aus den ermittelten Werten könnte das Ausgangsbild wiederhergestellt werden, die Werte sind lediglich eine maschinenlesbare Transformation des Ausgangsbildes, die dann im Trainingsvorgang verwendet wird. Die Art der Repräsentation ist stark abhängig von der Art der verwendeten Ausgangsdaten und dem geplanten Vorgehen im Trainingsprozess.

Ein Blick in die Praxis offenbart, dass etwa eine in TensorFlow bzw. Keras übliche Vorgehensweise nicht einen Tensor für jedes einzulesende Bild erzeugt, sondern einen image_batch-Tensor der Form ( $<$ Anzahl Bilder $>$, <Anzahl Pixel vertikal $>,<$ Anzahl Pixel horizontal $>,<$ Farbkanäle $>$ ), der die Werte aller eingelesenen Bilder enthält. ${ }^{125}$ Auf das Beispielbild in 3.2 übertragen hätte der Tensor also die Form (1, 20, 20, 3). Dies stellt jedoch lediglich eine Möglichkeit dar, Daten einzulesen, die Umsetzung ist von den Vorlieben des Entwicklers und der eingesetzten Technologie sowie der Beschaffenheit der Trainingsdaten abhängig.

Im Anschluss an die Umwandlung in das Tensor-Format erfolgen möglicherweise noch weitere Anpassungsschritte: Für KNN empfiehlt TensorFlow beispielsweise die Umwandlung der RGB-Werte, die zwischen 0 und 255

124 Die Farbnamen dienen nur der Veranschaulichung der Pixelwerte.

125 Dies ist der Fall bei Verwendung der Funktion image_dataset_from_directory (...), vgl. https://www.tensorflow. org/tutorials/load_data/images (Stand: 22.02.2021). Diese Funktion erzeugt ein Dataset-Objekt, das unter anderem den image_batch-Tensor und den labels_batch-Tensor enthält. 
liegen, in eine Repräsentation die sich zwischen 0 und 1 bewegt. ${ }^{126}$ Dadurch entsteht ein neues Dataset, bzw. ein neuer Tensor, der die angepassten Werte enthält.

\section{Trainingsvorgang}

Im Rahmen des Trainingsvorgangs werden die zuvor eingelesenen und vorbereiteten Daten im Modell durchlaufen. An dieser Stelle wird von der Wiedergabe mathematischer Funktionen zur Erklärung des Trainingsvorgangs abgesehen, da diese nicht dem Verständnis zuträglich wären. Stattdessen erfolgt eine abstrakte Beschreibung.

Im Rahmen eines typischen Trainingsvorgangs laufen die folgenden Schritte $a b$ (der genaue Prozess ist davon abhängig, was mit welchen Mitteln mit dem Modell erreicht werden soll): ${ }^{127}$

Abhängig von der Wahl und der Art des ML-Modells wird zunächst jeweils ein Tensor eingelesen und dann durch das Modell ,geleitet“.

Für den Fall von KNN bedeutet das, dass für jeden Wert des eingelesenen Datums ein Inputneuron den Wert übergeben bekommt, ggf. analysiert und je nach Ergebnis der Analyse und Architektur des Netzes das Ergebnis an alle oder ausgewählte Neuronen der nächsten Schicht weiterleitet. Das weitergeleitete Ergebnis ist mithin unter anderem abhängig von der dem Neuron zugewiesenen Funktion, bzw. den dem Neuron zugewiesenen Werten für Gewicht und Bias (vgl. oben § 2 B.II.2.). Weitergegeben wird also nicht ein Teil des Trainingsdatums, sondern das Ergebnis einer Berechnung. Die Neuronen der letzten Schicht repräsentieren das Ergebnis des Durchlaufs: so könnte für überwachtes Lernen jedes Neuron der letzten Schicht einem Label entsprechen. Das Neuron, das in der letzten Schicht aktiviert wird, entspricht dem Label, das das KNN im aktuellen Zustand dem eingelesenen Trainingsdatum zuweisen würde. Um den Lerneffekt zu erzielen, erfolgt ein Abgleich des errechneten Ergebnisses mit dem im Voraus dem Trainingsdatum zugeordneten Label. Bei Abweichungen nimmt ein Lernalgorithmus Anpassungen der variablen Werte in den Neuronen im KNN vor. Dieser Optimierungsvorgang erfolgt so lange, bis entweder zum Beispiel eine gewisse

126 Vgl. https://www.tensorflow.org/tutorials/load_data/images\#standardize_the_data (Stand: 22.02.2021).

127 Vgl. z. B. Beschreibung auf https://pytorch.org/tutorials/beginner/blitz/neural_ networks_tutorial.html\#sphx-glr-beginner-blitz-neural-networks-tutorial-py (Stand: 22.02.2021). 
Fehlerquote unterschritten wird oder eine zuvor definierte maximale Anzahl Trainingsvorgänge durchgeführt wurde.

Die Einzelheiten dieser Vorgehensweise sind abhängig vom eingesetzten System, jedoch hier nicht weiter relevant. ${ }^{128}$ Bemerkenswert ist, dass die gesamte Vorgehensweise einen Optimierungsprozess darstellt, in dem statistische Funktionen zum Einsatz kommen und in dem nicht das gesamte Trainingsdatum gleichmäßig verwertet wird, sondern indem lediglich anhand der eingelesenen Trainingsdaten Optimierungen der Werte im Modell vorgenommen werden.

\section{B. Urheberrechtliche Relevanz des Einlese- und des Trainingsvorgangs}

Auch in urheberrechtlicher Hinsicht ist zwischen dem Einlesevorgang und dem Trainingsvorgang zu unterscheiden. Im Rahmen des ersten könnten urheberrechtlich erhebliche Vervielfältigungs- und Bearbeitungshandlungen angenommen werden (dazu sogleich), während zweite nur mit Referenzen bzw. Repräsentationen der bereits eingelesenen Daten arbeitet und diese in der Regel nicht weiter vervielfältigt. Auch für das TDM wurde festgestellt, dass die eigentliche automatisierte Auswertung der Daten keinen urheberrechtlich relevanten Vorgang darstellt ${ }^{129}$ (wobei davon ausgegangen wird, dass - sobald die Daten vorbereitet sind - im Rahmen der Auswertung keine Vervielfältigungen mehr vorgenommen werden). ${ }^{130}$

\section{Vervielfältigung, § 16 UrhG}

Im Rahmen des zuvor beschriebenen Einlesevorgangs ${ }^{131}$ könnten gem. $\S \S 15$ Abs. 1 Nr. 1, 16 UrhG dem Urheber vorbehaltene Vervielfältigungen entstehen. Ansatzpunkte für mögliche Vervielfältigungen sind das Einlesen der Daten in den Arbeitsspeicher, die sich daran anschließende Umwandlung

128 Eine mathematische und praktische Einführung gibt z. B. Nielsen, Neural Networks and Deep Learning; außerdem gut verständlich Patterson/Gibson, Deep Learning, S. 27 ff.; Goodfellow et al., Deep Learning Handbuch, S. 107 ff..

129 BT-Drs. 18/12329, S. 40.

130 Wandtke/Bullinger-Bullinger, PK UrhR, § 60d Rn. 15; Dreier/Schulze-Dreier, UrhG, § 60d Rn. 4; Erw.Gr. 8 DSM-RL nennt als u. U. erlaubnispflichtigen Vorgang „während des Vorgangs des Text und Data Mining“ die Normalisierung von Daten.

131 S. dazu $§ 3$ A.I.. 
in einen oder mehrere Tensoren sowie etwaige Anpassungen der Werte der Tensoren.

Vervielfältigungen sind ,körperliche Festlegungen, die geeignet sind, das Werk den menschlichen Sinnen auf irgendeine Weise unmittelbar oder mittelbar wahrnehmbar zu machen“. ${ }^{132}$ Nach dieser aus der Gesetzesbegründung hervorgehenden Definition der Vervielfältigung kommt es lediglich darauf an, dass die Festlegung geeignet ist, das Werk wahrnehmbar zu machen, und nicht darauf, ob dies auch beabsichtigt ist. Der Zweck der Vervielfältigung ist also nicht zu berücksichtigen.

Die Behandlung der vorübergehenden Festlegung im Arbeitsspeicher als Vervielfältigung ist umstritten. ${ }^{133}$ An dieser Stelle kann die Einordnung jedoch möglicherweise dahinstehen, wenn in einem der weiteren Schritte des Einlesevorgangs eine Vervielfältigung erfolgt.

Zu klären ist, ob die körperliche Festlegung des Werkes in Form der Umwandlung in einen Tensor eine Vervielfältigung darstellt. Diese Umwandlung erfolgt einzig zu dem Zweck, das Trainingsdatum für den ML-Prozess nutzbar zu machen. Bezweckt ist mithin nicht, dass diese umgewandelte Form nach Abschluss des Prozesses für den Menschen sichtbar ist. Wie jedoch eingangs festgestellt, ist der Zweck der Festlegung unerheblich, solange diese geeignet ist, das Werk den menschlichen Sinnen wahrnehmbar zu machen.

Grundsätzlich könnten die Tensoren in die ursprüngliche Form der Trainingsdaten zurückgewandelt werden. Es ist gerade das Ziel des Umwandlungsvorgangs, die Trainingsdaten in der Gestalt der Tensoren einzeln ansteuern zu können, und dabei alle relevanten Informationen, die in den Trainingsdaten enthalten sind, in Tensoren abzulegen. Besonders deutlich wird die Beziehung zwischen Originaldatum und Tensor bei der Umwandlung von Bildern: Hier werden regelmäßig die einzelnen Pixel untersucht und in den Tensoren abgelegt. ${ }^{134}$ Es ist möglich, den Einlesevorgang umzukehren und das Bild aus den Tensoren wieder sichtbar zu machen. ${ }^{135}$ Mithin sind die Tensoren

132 BT-Drs. IV/270, S. 47.

133 Vgl. OLG Hamburg ZUM 2001, 512, 513 - Roche Lexikon Medizin; Dreier/SchulzeSchulze, UrhG, § 16 Rn. 13; Loewenheim-Loewenheim, Handbuch Urheberrecht, $\S 20$ Rn. 11; eine Vervielfältigung ablehnend KG ZUM 2002, 828, 830: Der Zustand im Arbeitsspeicher sei von der „Fortdauer der Energieversorgung des Rechners“ abhängig, weshalb nicht von einer körperlichen Festlegung zu sprechen sei.

134 Vgl. dazu schon $\$ 3$ A.I.2..

135 Vgl. z. B. die Diskussion dazu hier https://github.com/pytorch/pytorch/issues/30655 (Stand: 22.02.2021). 
mittelbar dazu geeignet, die eingelesenen Daten für die menschlichen Sinne wahrnehmbar zu machen, eine Vervielfältigung liegt also vor. ${ }^{136}$

Zudem erfolgen möglicherweise Anpassungen der Werte in den Tensoren, um die Verarbeitung durch den gewählten Modelltyp zu optimieren, wie etwa die zuvor beschriebene Umwandlung der RGB-Werte in Werte zwischen 0 und 1, wofür ein neues Data set-Objekt erzeugt wird. ${ }^{137}$ Nach dem Abschluss dieses Vorgangs existieren drei Repräsentationen der Trainingsdaten: die Originaldateien, der aus den eingelesenen Daten erzeugte Tensor, sowie der Tensor mit den angepassten Werten. Beide Tensoren sind unter Verwendung entsprechender TensorFlow-Funktionen dazu geeignet, die Originalbilder darzustellen. ${ }^{138}$ Insofern liegen also - in dieser angenommenen Vorgehensweise - zwei Vervielfältigungsstücke von allen Trainingsdaten vor. Die Anzahl tatsächlich erfolgender Vervielfältigungen ist von der konkreten Umsetzung durch den Entwickler abhängig. Es ist aber davon auszugehen, dass im Rahmen des Einlesevorgangs mindestens eine Vervielfältigung erfolgt, ${ }^{139}$ nämlich wenn der Tensor der Trainingsdaten angelegt wird.

Dass im sich an die Einlese- und Umwandlungsvorgänge anschließenden Trainingsprozess keine urheberrechtlich relevanten Vervielfältigungen entstehen, wurde bereits eingangs festgestellt. ${ }^{140}$

\section{Bearbeitung oder Umgestaltung, § 23 S. 1 UrhG}

In dem dargestellten Einlese- und Umwandlungsprozess könnte nicht nur eine Vervielfältigung, sondern auch eine Bearbeitung gem. § $23 \mathrm{~S} .1 \mathrm{UrhG}$ entstehen. Im Rahmen einer Bearbeitung erfolgt eine Anpassung des Werks an andere Nutzungsformen (geläufig sind vor allem Übersetzungen von Schriftwerken in andere Sprachen, Verfilmungen etc.), wobei die Identität des Ausgangswerkes im Gegensatz zur Umgestaltung nicht verändert wird. ${ }^{141}$

136 Im Ergebnis so auch Deutscher Bundestag, Künstliche Intelligenz und Machine Learning. Eine urheberrechtliche Betrachtung, S. 7.

137 Vgl. dazu § 3 A.I.2..

138 Vgl. https://www.tensorflow.org/tutorials/load_data/images\#visualize_the_data_2 (Stand: 22.02.2021).

139 So auch Deutscher Bundestag, Künstliche Intelligenz und Machine Learning. Eine urheberrechtliche Betrachtung, S. 7; Spindler, GRUR 2016, S. 1113.

140 Vgl. §3 B., bei Fußnote 129.

141 Vgl. BT-Drs. IV/270, S. 51; Dreier/Schulze-Schulze, UrhG, § 23 Rn. 6. 
1. Dem Original dienende Funktion, Anpassung an andere Nutzungsformen

Zunächst müssten die im Rahmen des Einlese- und Umwandlungsvorgangs entstandenen Repräsentationen der Trainingsdaten eine ,dem Original dienende Funktion“ erfüllen, bzw. eine Anpassung an andere Nutzungsformen darstellen im Sinne einer Erweiterung der Verwertungsmöglichkeiten der Originale. ${ }^{142}$ Um bei dem Beispiel der Bilddatei zu bleiben: die ursprüngliche Nutzungsform könnte die Wiedergabe auf einem Bildschirm sein, die neue Nutzungsform der Einsatz des Bildes zum Training eines Machine LearningModells, wofür die Anpassung in Form der Umwandlung in einen Tensor erforderlich ist. Hinzu kommt als Kriterium für eine Bearbeitung eine „dem Original dienende Funktion “ dergestalt in Betracht, dass die Bearbeitung eine Verwendung des Werks ermöglicht, die über die bisherige Verwendung hinausgeht und einen weiteren Verwertungsmöglichkeitenkreis eröffnet. ${ }^{143}$ Dies ist hier der Fall: Das Bild, das bisher lediglich der Verwendung als Bilddatei zugänglich war (z. B. zur Betrachtung oder Einbindung in Präsentationen oder Dokumente) kann nun dazu beitragen, ein Modell maschinellen Lernens zu beeinflussen, indem das Modell im Trainingsvorgang die Werte der in Tensoren eingelesenen Trainingsdaten berücksichtigt. Die Umwandlung der Trainingsdaten in Tensoren (sowie auch die anschließenden Anpassungen für spezielle ML-Modelltypen) sind also nicht nur Vervielfältigungen, sondern erfüllen auch grundsätzlich die Anforderungen an eine Bearbeitung. ${ }^{144}$

\section{Freie Benutzung?}

Auch wenn die grundsätzlichen Voraussetzungen einer Bearbeitung vorliegen, könnte eventuell auch eine freie Benutzung im Sinne des § 24 UrhG vorliegen. Dafür müsste jedoch ein neues, eigenständiges, schutzfähiges Werk entstehen, wobei das Originalwerk lediglich als Anregung dienen darf. ${ }^{145}$ Hier entspricht jedoch der Tensor dem Ausgangswerk, die Identität des Originalwerks wird mithin nicht verändert, sondern der Algorithmus ,sieht“ genau das, was

142 Vgl. BT-Drs. IV/270, S. 51; Dreier/Schulze-Schulze, UrhG, § 23 Rn. 5.

143 Dreier/Schulze-Schulze, UrhG, § 23 Rn. 6.

144 Anders Deutscher Bundestag, Künstliche Intelligenz und Machine Learning. Eine urheberrechtliche Betrachtung, S. 7, die nur darauf abstellen, dass lediglich eine Formatänderung vorliege, durch die das Werk grundsätzlich nicht verändert wird.

145 Dreier/Schulze-Schulze, UrhG, § 24 Rn. 5, 7. 
ein Mensch sähe, wenn er das Bild betrachtete, man könnte gar von einer Transformation sprechen. Eine freie Benutzung gem. § 24 UrhG liegt mithin in Bezug auf den Einlese- bzw. Umwandlungsvorgang nicht vor. ${ }^{146}$

\section{Einwilligungserfordernis bei Veröffentlichung oder Verwertung}

$\mathrm{Zu}$ beachten ist weiterhin, dass $\S 23 \mathrm{~S}$. 1 UrhG eine Einwilligung des Urhebers nur für den Fall erfordert, dass die Bearbeitung veröffentlicht oder verwertet werden soll; eine Herstellung einer Bearbeitung kann demnach auch ohne Einwilligung erfolgen, ${ }^{147}$ sofern es sich bei dem Original nicht um ein Datenbankwerk ${ }^{148}$ oder ein Computerprogramm handelt, ${ }^{149}$ und sofern die Bearbeitung oder Umgestaltung nicht veröffentlicht bzw. verwertet werden soll.

\section{a) Bearbeitung der Trainingsdatenbank?}

$\mathrm{Ob}$ die Trainingsdaten einzeln eingelesen und umgewandelt werden oder ob - wie in $\S 3$ A.I.2. beschrieben - alle Trainingsdaten gemeinsam eingelesen und in einen einzigen Tensor (bzw. in zwei Tensoren: In der Regel werden die Trainingsdaten in Trainings- und Validierungsdaten unterteilt) eingelesen werden, ist wiederum von der individuellen Umsetzung abhängig. Wenn, wie beschrieben, alle Trainingsdaten eingelesen werden, und die Trainingsdatensammlung die Anforderungen an ein Datenbankwerk erfüllt, ${ }^{150}$ ist die Bearbeitung nach dem soeben Gesagten unabhängig von der Anschlussnutzung einwilligungspflichtig. Eine Differenzierung zwischen dem Gesamtbestand der Trainingsdaten und den einzelnen Bestandteilen ist

146 Im Diskussionsentwurf, der § 24 UrhG aufhebt, wird in § 23 UrhG die Voraussetzung aufgenommen, dass eine Bearbeitung oder andere Umgestaltungen insbesondere dann unfrei sind, wenn sie keinen hinreichenden Abstand zum verwendeten Werk wahren ( $\$ 23$ Abs. 1 S. 1 UrhG-DiskE), sodass diese Voraussetzung dann die Prüfung des ehemaligen § 24 UrhG erübrigt, vgl. BMJV, DiskE 06/2020.

147 Vgl. auch Dreier/Schulze-Schulze, UrhG, § 23 Rn. 16.

148 Dreier/Schulze-Schulze, UrhG, § 23 Rn. 24; im Diskussionsentwurf auch explizit in $\S 23$ Abs. 2 Nr. 4 UrhG-DiskE genannt.

149 Dreier/Schulze-Schulze, UrhG, § 23 Rn. 19.

$150 \mathrm{Ob}$ die Trainingsdatensammlung als Datenbankwerk zu qualifizieren ist, ist einzelfallbezogen zu klären. 
jedoch entbehrlich, wenn auch die Bearbeitung der einzelnen Bestandteile geschieht und einwilligungspflichtig ist.

b) Veröffentlichung oder Verwertung der Bearbeitung

Zu prüfen ist, ob im Anschluss an die Umwandlung im Rahmen des Trainingsbzw. Produktiveinsatzes maschineller Lernverfahren üblicherweise eine relevante Veröffentlichung oder Verwertung der Bearbeitung erfolgt.

\section{aa) Veröffentlichung}

Eine Veröffentlichung liegt vor, wenn die Bearbeitung anderen Menschen als der Person des Bearbeiters zugänglich gemacht wird. ${ }^{151}$ Die Verarbeitung des Tensors erfolgt in der Regel lediglich modellintern, die Bearbeitung bzw. Umgestaltung tritt dabei nicht aus dem Modell heraus. Nach Abschluss des Trainingsvorgangs ist es zwar nicht unüblich, dass das Modell veröffentlicht wird, allerdings nur in einer Form von Computerprogrammcode, Hyperparametern und errechneter Parameter, die nicht ohne weiteres einen Rückschluss auf die einzelnen Tensoren bzw. die Trainingsdaten zulässt. Eine Veröffentlichung der Bearbeitung dergestalt, dass die Bearbeitung für (andere) Menschen wahrnehmbar zugänglich gemacht wird, ist also nicht anzunehmen.

\section{bb) Verwertung}

Möglicherweise liegt in der Verwendung der Umwandlung in Gestalt der Tensoren jedoch eine Verwertung. Welche Handlungen den Verwertungsrechten unterfallen, ergibt sich aus $\S 15$ UrhG. Dazu zählen demnach u. a. Vervielfältigung und Verbreitung, aber auch andere denkbare Verwertungsund Nutzungsarten, ${ }^{152}$ letztendlich also alles, was über die reine Rezeption hinausgeht. Es ist nicht auszuschließen, dass im weiteren Verlauf der Vorbereitung des Trainingsvorgangs zumindest einige Vervielfältigungen der Bearbeitung erfolgen, jedoch hängt dies von der individuellen Umsetzung

151 Dreier/Schulze-Schulze, UrhG, § 23 Rn. 17.

152 Dreier/Schulze-Schulze, UrhG, § 23 Rn. 18. 
durch den Entwickler ab. Insbesondere wenn die Aufbereitung der Trainingsdaten in einem vorgelagerten Prozess erfolgt, die Umwandlungen also für eine spätere Verwendung gespeichert und sodann erneut eingelesen werden, schließen sich Vervielfältigungen an die Bearbeitung an.

\section{c) Zwischenergebnis}

In der Konsequenz wäre dann die Umwandlung in das Tensorformat in jedem Fall einwilligungspflichtig, wenn dem Trainingsdatenbestand insgesamt Datenbankwerkqualität zukommt, und in der Regel einwilligungspflichtig, wenn auf die einzelnen Trainingsdaten abzustellen ist und kein Ausnahmetatbestand greift. Ein solcher liegt nach $\S 23$ S. 3 UrhG beispielsweise vor, wenn die Bearbeitung bzw. Umgestaltung im Rahmen eines Vorgangs des Text- und Data Mining ( $\$ 60 \mathrm{~d}$ UrhG) erfolgt. Auf die Ausnahmetatbestände wird in $\S 4$ eingegangen.

III. Zusammenhang zwischen Trainingsdaten und Output generativer Modelle

Angedacht werden könnte auch, ob im Falle von generativen Modellen, ${ }^{153}$ mit Output, der dem Format der Trainingsdaten entspricht (also zum Beispiel die Erzeugung von Bildern, wenn als Trainingsdaten auch Bilder verwendet werden), dieser Output eine Bearbeitung oder gar Vervielfältigung der Trainingsdaten darstellt. Nach dem Charakter des Trainingsvorgangs scheidet insbesondere eine Vervielfältigung schon deshalb aus, weil das Ergebnis nicht aus einer schlichten Kopie der Trainingsdaten entsteht, sondern diese lediglich als Datenbasis für komplexe Berechnungen verwendet werden.

Zudem ist zu unterscheiden zwischen Trainingsdaten, die eingesetzt werden, um das Modell zu trainieren, und eventuellen Inputdaten, die dem fertigen Modell zugeführt werden. Dies wird besonders deutlich am Beispiel des Google DeepDream-Systems: In der fertig trainierten Variante kann dem System durch den Benutzer ein Bild zur Verfügung gestellt werden, das daraufhin durch das Modell analysiert wird. Der Output gleicht dann in der Regel sehr stark dem Input, mit dem Unterschied, dass DeepDream Formen und Figuren in das Bild ,hineininterpretiert“" (vgl. für ein Beispiel

153 Vgl. oben $§ 2$ B.II.3.. 
Abbildung 10.2 und weitere Erläuterungen zum System in $\S 10$ C.I.). ${ }^{154}$ Die Trainingsdaten wirken sich nur insofern aus, als sie beeinflussen, welche Formen und Figuren in dem vom Benutzer gestellten Bild erkannt und hervorgehoben werden. Auch spiegeln sich dann nicht einzelne Trainingsdaten im Output wieder, sondern aus mehreren Trainingsdaten mit dem gleichen Label erkannte allgemeine Strukturen. Es erfolgt also gerade keine exakte Wiedergabe der Trainingsdaten im Output, und das vom Benutzer bereitgestellte Bild dominiert das Ergebnis.

Die Entwicklung eines Systems, das vollständige Trainingsdatenbilder in Ausgabedaten wiedergibt, ist zwar denkbar. Dies dürfte jedoch Spezialfälle darstellen, die dann einzelfallbezogen zu untersuchen sind. Gängiger ist das Training etwa anhand von Bildern eines bestimmten Malers, mit dem Ziel, ein neues Bild im Stile dieses Malers zu erzeugen. ${ }^{155}$ Wenngleich die erzeugten Ergebnisse stilistisch den Trainingsdaten ähneln, geben sie diese doch nicht wieder, sondern stellen das Ergebnis einer Berechnung aus der Analyse verschiedener Aspekte der Trainingsdaten dar. So könnte etwa die im Ergebnis abgebildete Nase den Mittelwert aller Nasen der Trainingsdaten wiedergeben, wenn das Modell so konfiguriert wurde.

Auch kann in dem Output keine Vervielfältigung oder Bearbeitung des aus der Umwandlung des Trainingsinputs entstandenen Tensors gesehen werden, denn - wie im Rahmen der Beschreibung des technischen Vorgangs dargestellt - der Tensor selbst wird im Trainingsprozess nicht weiter vervielfältigt oder bearbeitet, sondern lediglich als Berechnungs- bzw. Optimierungsgrundlage verwendet.

IV. Exkurs: Manipulierte ML-Modelle

Eine differenziertere Betrachtung wird erforderlich im Kontext manipulierter ML-Systeme. Es folgt eine knappe Erläuterung, weshalb es unter Umständen doch zu diskutieren ist, ob ein ML-Modell Vervielfältigungen von Trai-

154 In der Regel stellt aber der Urheber oder ein Berechtigter das Ausgangsbild zur Verfügung, sodass sich keine urheberrechtlichen Fragestellungen ergeben.

155 Vgl. z. B. das Projekt Next Rembrandt, https://www.nextrembrandt.com (Stand: 22.02.2021). 
ningsdaten enthält. Daran schließt sich eine Auswertung der Folgen auf die urheberrechtliche Betrachtung an. ${ }^{156}$

Unter einem manipulierten ML-Modell versteht diese Arbeit Modelle maschinellen Lernens, die in der Regel in der Cloud (Machine Learning as a Service, MLaaS) bereitgestellt werden, sodass der auszuführende Code für den Benutzer weder einsehbar noch kontrollierbar ist. Machine Learning as a Service beschreibt eine Dienstleistung, die es auch Benutzern ohne die erforderlichen Hardwarekapazitäten ermöglicht, Machine Learning für ihre Anwendungszwecke einzusetzen. Der Benutzer wählt dafür einen Anbieter und ein ML-Modell, das seinen Bedürfnissen entspricht, und stellt die zuvor gesammelten Trainingsdaten bereit.

Für (böswillige) Anbieter von MLaaS eröffnet das diverse Angriffsmöglichkeiten. Selbst wenn der Anbieter auf die Trainingsdaten, die der Benutzer hochlädt, nicht direkt zugreifen kann, bestehen für den Anbieter Möglichkeiten, die Trainingsdaten im Trainingsvorgang zu erfassen und in den Modellen selbst zu speichern, und damit etwa sensible Informationen auszulesen oder vom Kunden aufwendig zusammengestellte Trainingsdaten abzugreifen und für eigene Zwecke zu nutzen. Es gibt bereits zahlreiche Ansätze zur Umsetzung solcher Angriffe, exemplarisch seien hier nur die Capacity Abuse Attack, ${ }^{157}$ die Sign Encoding Attack ${ }^{158}$ sowie die Least Significant Bit At$t_{a c k}{ }^{159}$ genannt. Letztere wird für ein besseres Verständnis der diskutierten Problematik im Folgenden dargestellt.

\section{Beispiel: Least Significant Bit Attack}

Wie zuvor bereits beschrieben, bestehen ML-Modelle im Wesentlichen aus Struktur- bzw. Architekturvorgaben (Hyperparameter) und Parameterwerten (Weights / Biases). Letztere werden im Rahmen des Trainingsprozesses optimiert. Vermeintlich um eine möglichst hohe Präzision zu erreichen, wird für diese Werte in der Regel das Fließkommazahlenformat verwendet, das eine

156 Die folgenden Ausführungen wurden bereits veröffentlicht im Rahmen der DSRIHerbstakademie 2020, von Maltzan/Käde, Algorithmen, die nicht vergessen - Model Inversion Attacks und deren Bedeutung für den Schutz der Daten und der Urheberrechte, S. 505 ff. sowie in Käde/von Maltzan, InTeR 4 2020, $201 \mathrm{ff} .$.

157 Song/Ristenpart/Shmatikov, Machine Learning Models that Remember Too Much, 587, 593.

158 Dies., Machine Learning Models that Remember Too Much, 587, $591 \mathrm{f..}$

159 Dies., Machine Learning Models that Remember Too Much, 587, 590 f.. 
Zahl unter Verwendung von 32 Stellen (Bits) - Nullen und Einsen - darstellt. Der Wert 1,5 würde im Fließkomma-Binärformat wie folgt dargestellt: ${ }^{160}$

00111111110000000000000000000000

Dabei wirken sich allerdings Änderungen an den hinteren Bits nur marginal auf die Änderung des Wertes der Zahl aus: So entspricht

00111111110000000000000001001100

dem Dezimalwert 1,50000905991. Es ist also möglich, die hinteren - für den Zahlenwert weniger relevanten - Stellen nach Belieben zu verändern, ohne dass das Modell spürbar verändert wird. Im Beispiel können die hinteren Bits auch als Binärwert des Buchstaben „L“ (01001100) interpretiert werden.

Bis zu 20 Bits je Parameter können auf diese Weise verändert werden, bevor die Genauigkeit des Modells signifikant abnimmt. ${ }^{161}$

Die Least Significant Bit-Methode setzt genau an dieser Stelle an. Der Code, der für das Training mit den Nutzertrainingsdaten abläuft, liest die Daten ein, wandelt sie in Binärformat-Zeichenketten um und teilt die entstandene Zeichenkette in beispielsweise 20-Bit-Pakete. Diese werden, nachdem das Training abgeschlossen ist, auf die zur Verfügung stehenden Parameter verteilt und die letzten 20 Bits jedes Parameters entsprechend angepasst. ${ }^{162}$ Da das Modell ansonsten wie vom Nutzer erwartet trainiert wird, merkt dieser in der Regel nichts von dem Vorgang. Wenn der Nutzer das Modell im Anschluss wiederum frei zur Verfügung stellt, ${ }^{163}$ kann der Angreifer aus den von ihm modifizierten Parametern die Trainingsdaten rekonstruieren.

160 Binär dargestellte Fließkommazahlen unterscheiden sich von „,reinen“ Binärformatzahlen dadurch, dass sie auch das Vorzeichen und die Nachkommastellen berücksichtigen. Ein ganzzahliger Wert bräuchte zur Darstellung einer Eins im 32-Bit-Format nur das hinterste Bit: 00000000000000000000000000000001 , der Zwei entspräche 00000000000000000000000000000010 . Zur einfachen Umwandlung von Dezimalzu Binärformat vgl. z. B. https://www.h-schmidt.net/FloatConverter/IEEE754de. html (Stand: 22.02.2021).

161 Song/Ristenpart/Shmatikov, Machine Learning Models that Remember Too Much, 587, 595.

162 Dies., Machine Learning Models that Remember Too Much, 587, 595.

163 Für diese Fälle wird von „White-Box-Angriffen“ gesprochen, denn dem Angreifer steht zur Vollendung des Angriffs das gesamte Modell samt aller Werte zur Verfügung, vgl. auch Fredrikson/Jha/Ristenpart, Model Inversion Attacks That Exploit Confidence Information and Basic Countermeasures, 1322 und Song/Ristenpart/ Shmatikov, Machine Learning Models that Remember Too Much, 597. 


\section{Urheberrechtliche Bewertung}

Song/Ristenpart/Shmatikov haben gezeigt, dass es durch diese Manipulationen möglich ist, Trainingsdaten, die in Form von Bildern vorliegen, beinahe vollständig wiederherzustellen, und zeigen dies anhand eines Vergleichs der Originalbilder mit den rekonstruierten Versionen. ${ }^{164}$ Die rekonstruierten Bilder gleichen den Trainingsdaten dermaßen, dass tatsächlich davon gesprochen werden könnte, dass das manipulierte Modell eine Vervielfältigung im Sinne des Urheberrechts enthält. Die Modelle fungieren dann - ähnlich wie eine Festplatte, auf der Daten fragmentiert abgelegt werden - als Speichermedien für die abgegriffenen Daten. Noch einmal zur Verdeutlichung: Vervielfältigung im Sinne des Urheberrechts ist

jede körperliche Festlegung eines Werkes, die geeignet ist, das Werk den menschlichen Sinnen auf irgendeine Art mittelbar oder unmittelbar wahrnehmbar zu machen. ${ }^{165}$

Dafür genügt, dass die Vervielfältigung auf einem Datenträger gespeichert wird, der sodann dazu genutzt werden kann, das Werk wahrnehmbar zu machen - so wie es auch bei CDs und Festplatten der Fall ist. ${ }^{166}$ Nach dem Auslesen der wie beschrieben gespeicherten Informationen können die Ausgangswerke wieder wahrgenommen werden. Eine tiefergehende Betrachtung ist hier auch nicht erforderlich - schließlich liegt es auch schon aufgrund der Zielrichtung des Angreifers auf der Hand, dass genau die Vervielfältigung der Trainingsdaten beabsichtigt ist, um später Nutzen daraus ziehen zu können. Eine Bearbeitung ( $\$ 23 \mathrm{~S} .1 \mathrm{UrhG}$ ) hingegen liegt nicht vor und ist auch nicht beabsichtigt, Ziel ist die möglichst originalgetreue Reproduktion des Ausgangszustands. Festzuhalten ist: ML-Modelle können Vervielfältigungen enthalten, es bedarf dazu aber in aller Regel entsprechender Anstrengungen, um genau das zu erreichen.

\section{Zusammenfassung}

Nach der technischen Analyse und der erfolgten urheberrechtlichen Betrachtung ergibt sich, dass zwischen dem Einlese- und Aufbereitungsvorgang zum

164 Vgl. die Grafiken dazu in Song/Ristenpart/Shmatikov, Machine Learning Models that Remember Too Much, 587, 596.

165 RGZ 107, 277; BGHZ 17, 267, 269 f.; Dreier/Schulze-Schulze, UrhG, § 16 Rn. 6.

166 Dreier/Schulze-Schulze, UrhG, § 16 Rn. 7. 
einen und dem Trainingsvorgang zum anderen zu unterscheiden ist. Der reine Trainingsvorgang bleibt dabei frei von urheberrechtlich relevanten Handlungen, da hier nur Berechnungen bzw. Optimierungen stattfinden. Einer genaueren Untersuchung wurde dagegen der Einlese- und Aufbereitungsvorgang unterzogen. In dessen Rahmen werden regelmäßig Vervielfältigungshandlungen und teilweise auch Bearbeitungen vorgenommen. Es bleibt im Folgenden zu prüfen, ob diese von Ausnahmetatbeständen erfasst sind. 


\section{$\S 4$ Mögliche Ausnahmetatbestände}

Nachdem festgestellt wurde, dass grundsätzlich potenziell sowohl Vervielfältigungen als auch Bearbeitungen bzw. Umgestaltungen im Rahmen des Einlese- und Datenvorbereitungsvorgangs entstehen können, ist zu untersuchen, welche der bestehenden Ausnahmetatbestände die urheberrechtlich relevanten Handlungen dennoch einwilligungsfrei gestatten könnten. An dieser Stelle ist anzumerken, dass das UrhG aktuell einem umfassenden Änderungs- und Aktualisierungsprozedere unterliegt. Seit einem Entwurf vom 15. Januar 2020 war absehbar, dass ein $\S 44 b$ UrhG eingeführt wird, der die Anwendung des $\S 44 a$ UrhG im Kontext des Text und Data Mining endgültig unnötig macht, sowie dass u. a. eine Änderung des $§ 60 \mathrm{~d}$ UrhG erfolgt; beides dient der Umsetzung des Art. 3 DSM-RL. ${ }^{167}$ Die Betrachtung differenziert daher zwischen dem zum Stand der Bearbeitung aktuellen Gesetzesstand und die 2021 zu erwartenden Änderungen durch die Umsetzung der DSM-RL.

\section{A. Situation de lege lata}

Aktuell besteht eine Regelung zum TDM in $\S 60 \mathrm{~d}$ UrhG, darüber hinaus existiert ein Ausnahmetatbestand für vorübergehende Vervielfältigungshandlungen in $\S 44 \mathrm{a}$ UrhG. Folgend wird untersucht, ob diese TDM-Vorschriften auch auf den ML-Prozess angewendet werden können sowie welche Folgen sich daraus ergeben.

I. Zulässigkeit sowohl der Vervielfältigung als auch der Bearbeitung gem. § 60d UrhG?

Möglicherweise sind die Grundsätze des TDM gem. $\S$ 60d ff. UrhG auch auf maschinelle Lernverfahren anwendbar. Dies würde den Einlesevorgang und die Aufbereitung urheberrechtlich geschützter Daten zumindest in der

167 BMJV, DiskE 01/2020, S. 14. 
nichtkommerziellen wissenschaftlichen Forschung ${ }^{168}$ ermöglichen. Die sogenannte „Text- und Data Mining-Schranke“ soll gerade die flüchtigen Vervielfältigungen und Bearbeitungen, die im Rahmen automatisierter Auswertung von Datensammlungen zwangsläufig erfolgen, erlauben, sofern das TDM im Rahmen der nichtkommerziellen Forschung vorgenommen wird. ${ }^{169}$

Eine solche automatisierte Auswertung von Datensammlungen ist auch in der Datenverarbeitung im Prozess des maschinellen Lernens zu sehen; wie schon oben (§ 2 B.IV.1.) erläutert, liegt gerade darin die Schnittmenge von TDM und ML.

Auch in der „Strategie Künstliche Intelligenz“ der Bundesregierung ${ }^{170}$ wird TDM als Grundlage für maschinelles Lernen eingeordnet. ${ }^{171}$ Insbesondere wird dort festgehalten: „The right to read is the right to mine." ${ }^{172}$ Dies würde bedeuten: Sobald ein rechtmäßiger Zugang zu Werken besteht, ist das „Mining“ - also die automatisierte Verarbeitung z. B. zur Mustererkennung - gestattet, und damit auch die anfallenden urheberrechtlich relevanten Handlungen.

Fraglich und diskussionsbedürftig ist welche Handlungen dem MiningProzess zugerechnet werden. Gehört dazu nur der Trainingsprozess (also der eigentliche Vorgang der Informationsgewinnung) oder auch das Einlesen und Aufbereiten der Daten? In der oben zitierten KI-Strategie der Bundesregierung erfolgt hierzu keine Klarstellung. In der Gesetzesbegründung des $\S 60 d$ UrhG findet sich jedoch Folgendes:

„Die Reform regelt erstmals das Text und Data Mining, bei dem eine Vielzahl von Texten, Daten, Bildern und sonstigen Materialien ausgewertet werden, um so neue Erkenntnisse zu gewinnen. Die Vorschrift erlaubt insbesondere die mit dieser Methode einhergehenden Vervielfältigungen, sofern diese in urheberrechtlich relevanter Weise das Vervielfältigungsrecht berühren, $[\ldots]^{\star 1173}$

Die Formulierung „mit dieser Methode einhergehende Vervielfältigungen“ lässt darauf schließen, dass auch vorbereitende Maßnahmen (im Sinne des Einlesens und Aufbereitens der Trainingsdaten) von der Erlaubnis des $\S 60 \mathrm{~d}$ UrhG erfasst sein sollen.

168 Nach aktuellem Stand - die zu erwartenden Änderungen werden in § 4 B. erläutert und ausgewertet.

169 Vgl. BT-Drs. 18/12329, S. 22.

170 Bundesregierung, Strategie Künstliche Intelligenz der Bundesregierung.

171 Vgl. Dies., Strategie Künstliche Intelligenz der Bundesregierung, S. 40.

172 Dies., Strategie Künstliche Intelligenz der Bundesregierung, S. 40.

173 BT-Drs. 18/12329, S. 22. 
Damit gestattet $\S 60 d$ UrhG nach aktuellem Stand die im Rahmen des gesamten ML-Prozesses anfallenden Vervielfältigungen. Dies muss grundsätzlich auch für Bearbeitungen gelten, ${ }^{174}$ eine diesbezügliche Klarstellung erfolgt in $\S 23 \mathrm{~S} .3$ UrhG, der festlegt, dass ausschließlich technisch bedingte Änderungen eines Werkes im Rahmen des TDM im Sinne des § 60d UrhG nicht von $\S 23 \mathrm{~S} .1$ und 2 UrhG erfasst werden. ${ }^{175}$ Die zuvor angenommenen Bearbeitungen, die im Einlese- und Vorbereitungsvorgang anfallen können, erfolgen zu dem Zweck, die Daten für das ML-Modell optimal verarbeitbar zu gestalten, und sind mithin technisch bedingt. ${ }^{176}$

Auch für generative Modelle ${ }^{177}$ ergibt sich übrigens nichts anderes, selbst wenn hier auf den ersten Blick die Auswertung von Daten gegenüber der Synthetisierung von Daten im Hintergrund zu stehen scheint: Die Vervielfältigungshandlungen für den Trainingsprozess finden im Voraus für die Vorbereitung des diskriminierenden Parts statt. Dieser lernt, die charakteristischen Merkmale der Daten zu erkennen, die ihm zugeführt werden. Die für das TDM übliche Analyse und Auswertung findet also vor dem Einsatz und Training des generativen Parts statt, ist aber integraler Bestandteil.

\section{Zulässigkeit der Vervielfältigung gem. § 44a UrhG?}

Für die Einsatzbereiche außerhalb der nichtkommerziellen Forschung könnte nach dem aktuellen Gesetzesstand § 44a UrhG das Training der ML-Modelle ermöglichen. Möglicherweise ist die Vervielfältigung im Rahmen des Einlesevorgangs nach $\S 44 a \mathrm{Nr}$. 2 UrhG zulässig (wobei dann im weiteren Verlauf zu klären wäre, ob dies auch auf die Bearbeitung anwendbar ist). Dazu müsste die Vervielfältigung flüchtig oder begleitend sein, einen integralen und wesentlichen Teil eines technischen Verfahrens darstellen und ihr einziger Zweck müsste es sein, eine rechtmäßige Nutzung des vervielfältigten Werkes zu ermöglichen. Darüber hinaus dürfte die Vervielfältigung keine eigenständige wirtschaftliche Bedeutung haben.

174 So wohl auch Dreier/Schulze-Dreier, UrhG, § 60d Rn. 1.

175 Wandtke/Bullinger-Bullinger, PK UrhR, § 60d Rn. 14.

$176 \mathrm{Vgl}$. zu den möglicherweise anfallenden Bearbeitungen $\S 3$ B.II..

177 Vgl. zur Erklärung § 2 B.II.3.. 


\section{Flüchtige Vervielfältigungen}

Die Vervielfältigungen der Trainingsdaten im Rahmen des Einlesens und der Aufbereitung dürften in der Regel ohne weiteres als flüchtige Vervielfältigungen einzuordnen sein, denn sie werden erst bei Start des Programmablaufs erzeugt und existieren nicht über den Programmablauf hinaus.

\section{Rechtmäßige Nutzung}

Die Aufbereitungsvorgänge und der Machine-Learning-Trainingsprozess müssten rechtmäßige Nutzungen der flüchtig vervielfältigten Daten darstellen. Eine Nutzung ist dann rechtmäßig, ,wenn sie [...] vom jeweiligen Rechtsinhaber erlaubt ist, oder [..] im Rahmen gesetzlicher Schrankenbestimmungen zulässig und auch sonst nicht durch Gesetze beschränkt ist." ${ }^{178}$ Mit der Einstellung der Bundesregierung - „The right to read is the right to mine“179 müsste der ML-Prozess grundsätzlich als rechtmäßige Nutzung einzuordnen sein. Dies setzt aber voraus, dass ein ,right to read“, also ein rechtmäßiger Zugang zu den Daten, besteht.

\section{Vorübergehende Vervielfältigung rechtswidriger Quellen}

Ob § 44a UrhG auch die vorübergehende Vervielfältigung rechtswidriger Quellen privilegiert, wenn die sich daran anschließende Nutzungshandlung urheberrechtsfrei ist, ist umstritten. ${ }^{180}$ Der EuGH differenziert grundsätzlich zwischen rechtmäßigem und rechtswidrigem Zugang zu der zu vervielfältigenden Quelle, ${ }^{181}$ während die Rechtsprechung in Deutschland eine Privilegierung durch $\S 44 \mathrm{a}$ UrhG bisher wohl nur für den Fall ablehnt, dass die Rechtswidrigkeit der Quelle für den Nutzer offensichtlich ist (Anwendungsbereich ist insbesondere das Video-Streaming, wobei zu klären war, ob die Nutzer einer Plattform, die offensichtlich rechtswidrig kostenlosen Zugang

178 Dreier/Schulze-Dreier, UrhG, § 44a Rn. 8; Erw.gr. 33 InfoSoc-RL.

179 Bundesregierung, Strategie Künstliche Intelligenz der Bundesregierung, S. 40.

180 Dreier/Schulze-Dreier, UrhG, § 44a Rn. 8.

181 Vgl. EuGH C-527/15 Rn. 61 - Stichting Brein (Filmspeler); Dreier/Schulze-Dreier, UrhG, § 44a Rn. 8. 
zu Filmen bereitstellen, sich bzgl. des Streamings im eigenen Browser auf $\S 44$ a UrhG berufen können). ${ }^{182}$

Im Zuge der Umsetzung der DSM-RL wurden erneut Stimmen laut, die eine Klarstellung dahingehend fordern, dass eine automatisierte Auswertung aufgrund der urheberrechtlichen Neutralität des Vorganges - und der Möglichkeit eines Rückgriffs auf $\S 44 a$ UrhG - auch solcher Daten möglich sein müsse, zu denen der Zugang nicht rechtmäßig erlangt wurde - dies sei insbesondere im Hinblick auf investigativen Journalismus und Whistleblowing unerlässlich. ${ }^{183}$ Hier wird allerdings wohl zu differenzieren sein. So sind etwa ML-Anwendungen denkbar, die während des Modelleinsatzes weiterlernen (sog. „Online-Learning“"184). Diese Systeme erfassen Trainingsdaten im laufenden Betrieb (etwa durch Auswertung von Nachrichtenwebseiten), wozu Vervielfältigungen der zu analysierenden Daten erforderlich sind, die aber nicht gespeichert werden (müssen), sodass insofern nur flüchtige Vervielfältigungen anfallen. Es kann nicht Sinn der Regelung sein, dass Verwender solcher Systeme Zugangsschutzbarrieren (wie sie zum Beispiel bei kostenpflichtigen Nachrichtenseiten eingesetzt werden) umgehen und sich anschließend auf den Ausnahmetatbestand des § 44a UrhG berufen können. Dies erscheint unter anderem aus wirtschaftlichen Gesichtspunkten nicht wünschenswert.

\section{Zwischenergebnis}

Der Ausnahmetatbestand des $\S 44 a$ UrhG hilft an dieser Stelle also nicht in jedem Fall fehlender Privilegierung durch $\S 60 d$ UrhG weiter. Selbst wenn in vielen Fällen ML-Vervielfältigungen als flüchtige Vervielfältigungen einzustufen sind, besteht mindestens Rechtsunsicherheit dahingehend, ob der Ausnahmetatbestand auch bei rechtswidrig erlangten Daten greift.

182 Dreier/Schulze-Dreier, UrhG, § 44a Rn. 8; vgl. z. B. LG Köln GRUR-RR 2014, 114, 115 - The Archive.

183 Wikimedia Deutschland, Stellungnahme DiskE 01/2020, S. 6.

184 Goodfellow et al., Deep Learning Handbuch, S. 310. 


\section{B. Situation nach zu erwartenden Änderungen durch die Umsetzung der DSM-Richtlinie}

Deutlich mehr Klarheit in Bezug auf die Regelung der Machine LearningHandlungen wird durch die Umsetzung aktueller europäischer Rechtsakte erwartet. Es folgt eine knappe Analyse der umzusetzenden Vorgaben, im Anschluss daran eine Erörterung der geplanten nationalen Umsetzung, die danach in Bezug auf Machine Learning ausgewertet wird.

\section{Was ist umzusetzen?}

Auf EU-Ebene wurden im Jahr 2019 mit der DSM-RL in deren Artikeln 2, 3 und 4 auch einige Regelungen zum Text- und Data Mining verabschiedet, die allerdings noch bis 2021 in nationales Recht umzusetzen waren. Unter anderem ist die TDM-Schranke nicht mehr explizit auf nichtkommerzielle Forschung beschränkt, sondern soll jedermann zumindest die automatisierte Auswertung und die dazu vorgenommenen Vervielfältigungshandlungen erlauben.

Artikel 2 Nr. 2 enthält eine Definition des TDM:

„Text und Data Mining bezeichnet eine Technik für die automatisierte Analyse von Texten und Daten in digitaler Form, mit deren Hilfe Informationen unter anderem aber nicht ausschließlich - über Muster, Trends und Korrelationen gewonnen werden können.“

Artikel 3 DSM-RL regelt TDM zum Zwecke der wissenschaftlichen Forschung, Artikel 4 sodann „Ausnahmen und Beschränkungen für das Text und Data Mining“. Letztere gelten nicht mehr nur für (nichtkommerzielle) Forschung, sondern erlauben ,zum Zwecke des Text und Data Mining vorgenommene Vervielfältigungen und Entnahmen von rechtmäßig zugänglichen Werken und sonstigen Schutzgegenständen“ (Art. 4 Abs. 1) ohne eine Beschränkung auf einen bestimmten Anwenderkreis.

Artikel 3 verlangt für Forschungsorganisationen und Einrichtungen des Kulturerbes, die die gesammelten Daten bzw. Korpora speichern und aufbewahren, hingegen nicht, dass diese Aufbewahrung für die Zwecke des TDM erforderlich ist - und privilegiert damit ebendiesen Anwenderkreis. „Forschungsorganisationen“ sind nach wie vor Einrichtungen, die ,in ihrer Tätigkeit nicht gewinnorientiert [sind] oder alle Gewinne in ihre wissenschaftliche Forschung [reinvestieren], oder im Rahmen eines von einem 
Mitgliedstaat anerkannten Auftrags im öffentlichen Interesse tätig [sind]“ (Art. 2 Nr. 1 a und b DSM-RL), also im wesentlichen die schon bisher privilegierte nichtkommerzielle Forschung.

\section{Wie erfolgt die Umsetzung?}

Die Umsetzung erfolgt im Rahmen eines „Gesetzes zur Anpassung des Urheberrechts an die Erfordernisse des digitalen Binnenmarkts“ (UrhG-E). Hierzu existieren zwei Diskussionsentwürfe des BMJV von Januar und Juni 2020, die in einem im Oktober 2020 veröffentlichten Referentenentwurf zusammengeführt wurden, ${ }^{185}$ aus dem sich auch schon das Schicksal der TDM-Regelungen (de lege lata und de lege ferenda) andeutete. Am 03. Februar 2021 wurde ein Regierungsentwurf beschlossen, der sich in Bezug auf die TDM-Regelungen nicht von dem Referentenentwurf aus Oktober 2020 unterscheidet. ${ }^{186}$ Das entsprechende Gesetz wurde am 04. Juni 2021 im Bundesgesetzblatt verkündet und ist am 07. Juni 2021 in Kraft getreten. ${ }^{187}$ Die Verkündung erfolgte nach Einreichung dieser Arbeit, daher entspricht im Folgenden „UrhG-E“ dem aktuell seit 07. Juni 2021 geltenden Urheberrechtsgesetz und „UrhG“ stellt den Stand vor der Umsetzung dar.

Die Umsetzung des Art. 4 DSM-RL erfolgt durch den neuen § 44b UrhGE, Art. 3 DSM-RL wird durch eine Neufassung des $\S 60$ d UrhG-E umgesetzt. Der neue $\S 44 b$ UrhG-E enthält im ersten Absatz eine Definition von Text und Data Mining, regelt im zweiten Absatz, welche Vervielfältigungen zulässig sind und räumt im dritten Absatz dem Rechtsinhaber auch die Möglichkeit einer Nutzungsuntersagung ein. ${ }^{188}$

Die Definition in $\S 44 b$ Abs. 1 UrhG-E, die die zuvor gefundene Definition $^{189}$ des TDM weiter präzisiert, entspricht inhaltlich im Wesentlichen der, die bereits in Art. 2 Nr. 2 DSM-RL enthalten ist: Danach ist Text und Data

185 BMJV, RefE 10/2020.

186 Bundesregierung, RegE 02/2021, S.13 f..

187 Gesetz zur Anpassung des Urheberrechts an die Erfordernisse des digitalen Binnenmarktes vom 31. Mai 2021, BGB1. 2021 Teil I Nr. 27, hrsg. am 04. Juni 2021, S. $1204 \mathrm{ff}$.

188 Bundesregierung, RegE 02/2021, S. 13; BMJV, DiskE 01/2020, S. 5; im Rahmen dieses Kapitels wird auf die Seitenzahlen der Entwürfe abgestellt, da das Gesetz erst nach Ende der Bearbeitung dieser Arbeit in Kraft getreten ist. Inhaltlich haben sich seit dem Regierungsentwurf in Bezug auf die hier diskutierten Passagen im verabschiedeten Gesetz keine Änderungen mehr ergeben.

189 S. oben $\S 2$ B.IV.1.. 
Mining die automatisierte Analyse von einzelnen oder mehreren digitalen oder digitalisierten Werken, um daraus Informationen insbesondere über Muster, Trends und Korrelationen zu gewinnen.

Eine Beschränkung der Zulässigkeit des TDM auf den Kreis nichtkommerzieller Forschung erfolgt nicht mehr. $\mathrm{Zu}$ beachten ist auch, dass diese explizite TDM-Regelung nicht mehr in den $\S \S 60$ a ff. UrhG (gesetzlich erlaubte Nutzungen für Unterricht, Wissenschaft und Institutionen), sondern im Abschnitt (allgemein) gesetzlich erlaubter Nutzungen zu finden ist. Damit wird dem Gedanken Rechnung getragen, dass TDM eben nicht mehr nur einem bestimmten Adressatenkreis vorbehalten sein soll, während alle anderen sich mit dem § 44a UrhG abfinden müssen - sondern der Erkenntnisgewinn durch TDM soll allen möglich sein, die das Potenzial dieser Technologie erkennen und sie einsetzen möchten.

Darüber hinaus wird zur Umsetzung des Art. 3 DSM-RL der § 60d UrhG-E überarbeitet. Dieser stellt nun eine Spezialregelung gegenüber § 44b UrhG-E für die Forschung dar, wobei das bisher strenge Kriterium der Nichtgewerblichkeit durch die differenziertere von der DSM-RL vorgegebene Charakterisierung der Berechtigten ersetzt wird. ${ }^{190}$

In § 44b UrhG-E verlangte der Entwurfstext im Januar 2020 noch die Erforderlichkeit der vorgenommenen Vervielfältigungen (,Zulässig sind Vervielfältigungen, sofern sie für das Text und Data Mining erforderlich sind“"), ${ }^{191}$ obgleich Artikel 4 DSM-RL die Erforderlichkeit der Vervielfältigungshandlungen nicht bedingt. Schon im Referentenentwurf war die Erforderlichkeit so nicht mehr Bestandteil des $\S 44 b$ Abs. 2 UrhG-E, dies ist auch im Regierungsentwurf übernommen worden. ${ }^{192}$ Satz 2 des § 44b Abs. 2 UrhG-E besagt allerdings nach wie vor, dass die Vervielfältigungen zu löschen sind, wenn sie für das TDM ,nicht mehr erforderlich“ sind. ${ }^{193}$

Möglicherweise kann hieraus dennoch die grundsätzliche Erforderlichkeit der Vervielfältigungen konstruiert werden, denn ,,nicht mehr erforderlich“ impliziert, dass zuvor eine Erforderlichkeit vorlag. Insofern ist davon auszugehen, dass diese textliche Änderung lediglich der Verbesserung der Lesbarkeit dienen sollte, es ist zu hoffen, dass diesbezüglich noch eine Klarstellung erfolgt. Anderenfalls könnten sich schwerwiegende Folgen für den ML-Entwicklungsprozess ergeben: Nicht immer ist Code effizient aufgebaut, mitunter werden etwa die geladenen Daten im Arbeitsspeicher in andere

190 Bundesregierung, RegE 02/2021, S. 14; BMJV, DiskE 01/2020, S. 6.

191 Dass., DiskE 01/2020, S. 5.

192 Bundesregierung, RegE 02/2021, S. 13.

193 Vgl. Dies., RegE 02/2021, S. 13. 
Variablen kopiert. Müssen die Entwickler bei jedem Arbeitsschritt darüber nachdenken, ob sie gerade eine unnötige Vervielfältigung vornehmen? Welcher Schaden würde den Urhebern aus diesen, nur im Code vorgenommenen und den Arbeitsspeicher nicht verlassenden, aber unnötigen Vervielfältigungshandlungen entstehen? An dieser Stelle ist aus Gründen der Rechtssicherheit für die Entwickler zu fordern, dass hier nicht zu engmaschige Anforderungen an die Erforderlichkeit der Vervielfältigungen oder andere urheberrechtlich relevante Handlungen gestellt werden.

$\S 60 d$ Abs. 1 UrhG-E bezieht sich auf $\S 44 b$ UrhG-E, und zwar nicht nur für die Begriffsdefinition, sondern explizit auch auf Zulässigkeit der Vervielfältigungen in $\S 44 \mathrm{~b}$ Abs. 2 S. 1 UrhG-E. § 44b Abs. 2 S. 2 UrhG-E ist für gem. § 60d Abs. 1 UrhG-E privilegiertes TDM nicht anwendbar. Daraus ergibt sich die Frage, ob dann auch das Kriterium der Erforderlichkeit der Vervielfältigungen in Satz 1 nicht mehr gegeben ist. An dieser Stelle bleibt die gesetzliche Regelung unklar.

Aus der Begründung geht nicht hervor, dass hier eine Abweichung von Art. 4 Abs. 1 DSM-RL beabsichtigt ist. Hier bleibt abzuwarten, ob diesbezüglich eine Überarbeitung zur Klarstellung erfolgt. Dies wäre zu begrüßen, denn die Beschränkung auf erforderliche (im Unterschied zu lediglich zum Zweck des TDM vorgenommenen) Vervielfältigungshandlungen bedingt unter Umständen ein aufwendiges Hinterfragen von Programmierpraktiken ${ }^{194}$ und Datensicherungsstrategien, während die Zweckbezogenheit ohne Weiteres bei allen im Programmcode angelegten Vervielfältigungen angenommen werden kann, sofern nicht offensichtlich andere Zwecke verfolgt werden.

Kritisiert wird an der neuen Fassung bisher unter anderem, dass lediglich die Verarbeitung von Daten geregelt sei, wohingegen keine Aussagen darüber getroffen würden, ob ,das Anzeigen und die Mitteilung der Ergebnisse einer solchen Verarbeitung“" erlaubt seien. ${ }^{195}$ Des Weiteren habe $\S$ 60d UrhG-AF auch die öffentliche Zugänglichmachung des Korpus bzw. der gesammelten Daten für zulässig erklärt, wohingegen eine solche Regelung in § 44b UrhG-E nicht enthalten ist. ${ }^{196}$

Dass auf TDM bei rein flüchtigen Vervielfältigungen auch weiterhin die hierfür bestehende Regelung anwendbar sein soll (in Deutschland also der

194 Zum Beispiel für den Fall, dass die Vervielfältigungen nicht im Prozess der Datensammlung, sondern während der Aufbereitung bei Programmstart bzw. zur Normalisierung (vgl. Erw.Gr. 8 DSM-RL) erfolgen.

195 Google, Stellungnahme DiskE 01/2020, S. 8.

196 Dass., Stellungnahme DiskE 01/2020, S. 8 f.. 
$\S 44 a$ UrhG), geht aus Erw.Gr. 9 DSM-RL hervor. Dies ist dem Entwurfstext nicht ohne Weiteres zu entnehmen, was ebenfalls bereits für Kritik sorgte. ${ }^{197}$

1. Welche Auswirkungen ergeben sich für den ML-Prozess?

$\mathrm{Zu}$ untersuchen sind noch die Abweichungen in der Behandlung des TDM bzw. die Auswirkungen der zu erwartenden Änderungen auf die Behandlung des ML-Prozesses. Zunächst ergibt die neue Regelung einen Unterschied an die Herangehensweise der Prüfung dergestalt, dass zum Einen nach der Anwendergruppe zu unterscheiden ist und zum anderen nach den fraglichen Handlungen: Denn wenn die Privilegien aus § 60d UrhG-E nicht in Anspruch genommen werden sollen, reichen unter Umständen die $\S \S 44 a$ und 44b UrhGE aus.

Einen Überblick über die zu erwartenden Änderungen verschafft Abbildung 4.1. Das Schema unterscheidet vier exemplarische Personengruppen (Privatperson („Citizen Science“), Einzelforscher, nicht-kommerziell handelnde und kommerziell handelnde Forschungsorganisationen) und analysiert die TDM-Prozessschritte hinsichtlich bestehender und zu erwartender urheberrechtlicher Regelungen. Die rechten Teilspalten stellen dabei jeweils den Stand nach Umsetzung der DSM-RL laut aktuellem Entwurf dar. Festzustellen ist, dass kommerzielle Forschung und Citizen Science urheberrechtlich gleich gestellt sind, und sich in Zukunft - wie zuvor der nichtkommerziellen Forschung vorbehalten - auch auf eine TDM-Schranke berufen können (§ 44b UrhG-E).

Warum Einzelforscher laut dem Entwurf (der so auch übernommen wurde) nicht mehr explizit zur Aufbewahrung der Vervielfältigungen befugt sind, geht aus der Entwurfsbegründung nicht hervor. Damit sind sie in dem Punkt gleichgestellt zur Citizen Science, womit gem. § 44b Abs. 2 S. 2 UrhG-E die Vervielfältigungen zu löschen sind, wenn sie für das TDM nicht mehr erforderlich sind. ${ }^{198}$

Übertragen auf den Prozess des maschinellen Lernens bleibt die oberste Zeile in Abbildung 4.1 außer Betracht, relevant sind vor allem die zweite und dritte Zeile. Auch die Nachnutzung des erstellten Korpus (Zeilen vier und fünf) liegen außerhalb des Fokus dieser Arbeit. Grundsätzlich kann sich in Zukunft ungeachtet der konkreten Umsetzung also jeder auf Ausnahme-

197 Wikimedia Deutschland, Stellungnahme DiskE 01/2020, S. 5 f..

198 Dies kritisierend Spindler, Kurz-Stellungnahme zum DiskE, S. 1. 


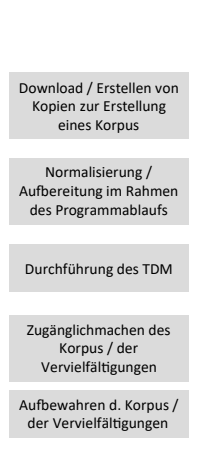

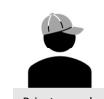
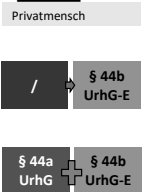

- frei -

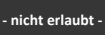

- nicht erlaubt -
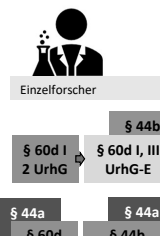

$\S 60 d, \S 44 b$
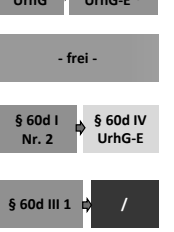

* Durch Verweis von § 60d UrhG
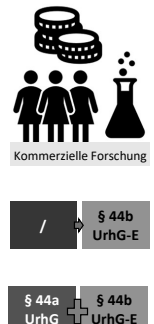

- frei -

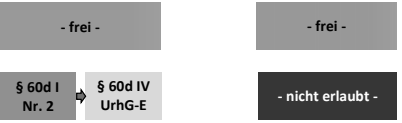

- nicht erlaubt -

Abbildung 4.1: Änderungen der TDM-Regelungen, Quelle: eigene Darstellung.

tatbestände berufen und ist nicht mehr auf den engen Anwendungsbereich flüchtiger Vervielfältigungen des $§ 44 \mathrm{a}$ UrhG beschränkt.

\section{Zusammenfassung}

Machine Learning ist (schon jetzt) auf jeglichen Daten zulässig, ungeachtet der Legitimität des Zugangs - sofern keine nicht lediglich flüchtigen Vervielfältigungen stattfinden. Bezüglich für den Zweck des Machine Learnings erfolgender Vervielfältigungen im Vorfeld und im Rahmen des MLProzesses kann sich in Zukunft jede/r auf die anwendbare TDM-Schranke in $\S 44 \mathrm{~b}$ UrhG-E berufen, wohingegen diese Schranke zum aktuellen Zeitpunkt noch der nichtkommerziellen Forschung vorbehalten ist. 


\section{$\S 5$ Ergebnis des zweiten Teils}

In diesem Teil der Arbeit wurde der Machine Learning-Prozess untersucht. Dabei konnten unterschiedliche Phasen der Datenverarbeitung identifiziert werden:

Die Trainingsdaten, aus denen die ML-Modelle lernen, werden zunächst eingelesen und dann für die Verarbeitung im Trainingsvorgang vorbereitet. Dabei erfolgt zum einen eine Umwandlung in ein für das ML-Programm lesbares Datenformat, zum Beispiel Tensoren, und zum anderen ggf. auch noch eine Anpassung oder Skalierung der Daten, um ein effizientes Training zu ermöglichen. Erst im Anschluss daran analysieren die ML-Modelle die Trainingsdaten im Trainingsprozess und optimieren währenddessen ihre Parameter.

Auch vor dem eigentlichen Machine Learning-Prozess müssen Data Scientists ggf. Maßnahmen treffen: Zum einen sind die passenden Trainingsdaten zu sammeln, diese sind (für das überwachte Lernen) mit Labels zu versehen; außerdem kann es zur Sicherung der Datenqualität etwa erforderlich sein, „Ausreißerdaten“ (also Daten, die für das zu untersuchende Merkmal eigentlich untypisch sind) zu entfernen oder eine möglicherweise Datensammlung immanente Voreingenommenheit oder implizite Vorurteile auszugleichen, indem Datenpunkte weggenommen oder hinzugefügt werden (so könnten zum Beispiel für die visuelle Erkennung eines ausgeübten Berufs einer abgebildeten Person geschlechterspezifische Verzerrungen in den Daten enthalten sein, weil von einer Berufsgruppe überdurchschnittlich viele Personen eines Geschlechts in den Trainingsdaten vertreten sind). Diese vorbereitenden Handlung liegen jedoch außerhalb der erklärten Grenzen dieser Arbeit und wurden daher nicht auf urheberrechtlich relevante Handlungen untersucht, stattdessen wurde auf den eigentlichen ML-Prozess fokussiert.

Auf die eingangs gestellten Fragen zurückkommend ist demnach Folgendes festzustellen: Hinsichtlich des Machine Learning-Prozesses ist also zu unterscheiden zwischen den vorbereitenden Handlungen, dem Einlesevorgang, der dann ggf. erfolgenden Anpassung der Daten sowie dem eigentlichen Training. Urheberrechtlich relevante Handlungen kommen insbesondere außerhalb des eigentlichen Trainings, also im Rahmen der Einlese- und Aufbereitungs- 
vorgänge, in Betracht. ${ }^{199}$ Für diese sind dann die geltenden und die mit der Umsetzung der DSM-RL kommenden TDM-Schrankenbestimmungen in den $\S \S 44 a, 44 b$ (UrhG-E) und 60d UrhG relevant und anwendbar. ${ }^{200}$ Trainingsdaten sind in der Regel nicht im fertigen Modell enthalten, es sei denn, das Modell wurde diesbezüglich manipuliert. ${ }^{201}$

$\mathrm{Zu}$ berücksichtigen ist jedoch, dass diese Einschätzung nur eine Momentaufnahme des aktuellen, untersuchten Technologiestandes darstellt. Der technische Fortschritt ist stets im Blick zu behalten.

199 S. oben $\S 3$ B..

200 S. zur Situation de lege lata und de lege ferenda oben $\S 4$.

201 S. zur Manipulation den Exkurs in $\S 3$ B.IV.. 


\section{Dritter Teil \\ Schutzmöglichkeiten für Modelle maschinellen Lernens im Urheberrecht}

Nachdem geklärt wurde, welche Voraussetzungen erfüllt sein müssen, um ML-Modelle rechtmäßig zu trainieren, stellt sich die Frage, wie die trainierten Modelle rechtlich geschützt sein könnten. Schließlich fließen viel Erfahrung, Arbeitszeit, Geld und technische Ressourcen in die Entwicklung effizienter und korrekt arbeitender Machine Learning-Modelle. ${ }^{202}$

In der Regel sind die trainierten Modelle das Ergebnis eines unter Umständen langwierigen Prozesses des Trainingsdatensammelns, -auswertens und -aufbereitens, der Modelleinstellung und -optimierung, des Modelltrainings, der Ergebnisauswertung und ggf. iterativen Überarbeitung. Daher sprechen sowohl die Wertschätzung der Arbeit der Entwickler maschineller Lernmodelle bzw. Systeme als auch der Innovationsanreiz für einen solchen Schutz.

Problematisch in der Diskussion um den Schutz von ML-Modellen scheint, dass es bisher im juristischen Diskurs noch nicht greifbar genug war, was ein trainiertes Modell ausmacht.

Schon bei der Betrachtung des Entstehungsprozesses eines trainierten Modells wird klar, dass hier eine nicht unerhebliche menschliche Leistung erforderlich ist, um zum gewünschten Ergebnis zu kommen. Es ist mitnichten lediglich ein mathematischer Rechenvorgang, der das trainierte Modell erzeugt, sondern es ist Erfahrung in Bezug auf die Auswahl von Trainingsdaten sowie insbesondere die Strukturierung des Modells erforderlich.

Wirtschaftlich gesehen, ist das trainierte Modell ein hochwertiges Werkzeug, das einerseits im Produktiveinsatz bestehen muss, andererseits aber ungeschützt - einfach kopiert und ausgebeutet werden kann.

Der urheberrechtliche Schutz des „Gehirns von KI“ - der Modelle maschinellen Lernens - wurde bereits in einigen Veröffentlichungen diskutiert

202 Eine Übersicht der Entwicklung weltweiter Investitionen in KI kann z. B. dem AI Index Report 2019 entnommen werden: Perrault et al., The AI Index 2019 Annual Report, S. 94 ff.; vgl. außerdem Graf, Multitalent für Sprache (Spektrum.de vom 11.08.2020). 
bzw. zumindest angerissen, ${ }^{203}$ jedoch bisher an keiner der Autorin bekannten Stelle ausreichend tiefgehend bzw. zutreffend mit Blick auf die tatsächlich verwendete Technologie durchleuchtet. Dessen nimmt sich dieser Teil der Arbeit an. Dazu werden die relevanten Bausteine von ML-Systemen identifiziert (s. § 6) und daraufhin auf ihre Schutzfähigkeit kategorisch untersucht (s. § 7).

Auch außerhalb der Vorschriften des Urheberrechtsgesetzes wird ein Schutz für ML-Modelle diskutiert. So wird etwa ein Schutz als Geschäftsgeheimnis im Sinne der GeschGeh-RL bzw. des GeschGehG angedacht, ${ }^{204}$ und auch das Wettbewerbsrecht könnte Anknüpfungspunkte ${ }^{205}$ bieten.

Vorgeschlagen wird zudem die Einführung eines Leistungsschutzrechts für computergenerierte Erzeugnisse (mit dem Ziel, die errechneten „Trai-

203 Ehinger/Stiemerling, CR 12 2018, 761 ff.; Hauck/Cevc, ZGE 11 2019, 135 ff.; Papastefanou, CR 4 2019, 209 ff.; Linke, GRUR Junge Wissenschaft 2019, 29 ff.; Nebel/Stiemerling, CR 1 2016, 61 ff.; Hartmann/Prinz, WRP 12 2018, 1431 ff.; Gomille, JZ Nr. 20 2019, 969, 970; Spindler, IIC 2019, 1049, 1050; Linke/Petrlik, GRUR Int. 2020, 39 ff.; Grätz, Künstliche Intelligenz im Urheberrecht; Iglesias Portela/Shamuilia/Anderberg, Intellectual Property and Artificial Intelligence: A Literature Review: EUR 30017 EN, S. 9;.

204 Ehinger/Stiemerling, CR 12 2018, 761, 769 erwägen einen Geschäftsgeheimnisschutz für „Trainingsergebnisse“ im Rahmen der GeschGeh-RL, ebenso sieht Gomille, JZ Nr. 20 2019, 969, 970 einen Schutz gem. §§ 2 ff. GeschGehG einschlägig; vgl. außerdem ausführlich Hauck/Cevc, ZGE 11 2019, 135, 163 f.; Söbbing, Fundamentale Rechtsfragen zur künstlichen Intelligenz. (AI Law), S. 14; einen Schutz für Modelle erwägend Apel/Kaulartz, RDi Nr.1 2020, 24, 29; insbesondere für ,durch ein deutlich höheres $\mathrm{Ma}$ an Autonomie bestimmter" neuronaler Netzwerke auf GeschGeh-Schutz verweisend Schricker/Loewenheim-Loewenheim/Leistner, Urheberrecht, $\S 2$ Rn. 41a, allerdings ist davon auszugehen, dass die Autoren nicht den Schutz neuronaler Netze an sich meinen, sondern Erzeugnisse - für diese wäre allerdings insbesondere im Zusammenhang der Erzeugung ,künstlerischer“ Werke fraglich, wie hier ein GeschGeh-Schutz anzubringen wäre: Denn die Komplexität der neuronalen Netze ändert nach hiesiger Ansicht nichts an der Zurechenbarkeit ihrer Erschaffung (also der Erschaffung der Netze) zum Entwickler.

205 Hauck/Cevc, ZGE 11 2019, 135, 166 f. nennen u. a. § 4 Nr. 3 UWG als Auffangtatbestand im Rahmen des lauterkeitsrechtlichen Nachahmungsschutzes. 
ningsergebnisse“ zu schützen). ${ }^{206}$ Naheliegend und bereits in der Praxis angekommen ist zudem der Schutz im Patentrecht. ${ }^{207}$

Diese Arbeit fokussiert jedoch auf diejenigen Schutzmöglichkeiten, die das UrhG bietet.

206 Ehinger/Stiemerling, CR 12 2018, 761, 769.

207 Ausführlich dazu Hauck/Cevc, ZGE 11 2019, 135 ff., die einen anwendungsbezogenen Patentschutz für möglich halten; gegen einen patentrechtlichen Schutz: Gomille, JZ Nr. 20 2019, 969, 970; Apel/Kaulartz, RDi Nr.1 2020, 24, 29, die ein Problem insbesondere in Bezug auf die erforderliche Technizität sehen; auf europäischer Ebene steigt die Zahl der (begehrten) Patentanmeldungen, vgl. Iglesias Portela/ Shamuilia/Anderberg, Intellectual Property and Artificial Intelligence: A Literature Review: EUR 30017 EN, S. 6; das Europäische Patentamt hat Richtlinien zur Patentierung von KI herausgegeben, vgl. z. B. https://www.epo.org/law-practice/legaltexts/html/guidelines2018/e/g_ii_3_3_1.htm (Stand: 22.02.2021) und Craglia et al., Artificial intelligence: A European perspective, S. 66. 


\section{§ 6 Technische Bestandsaufnahme}

Ein großer Teil der divergierenden Aussagen über den urheberrechtlichen Schutz von ML-Modellen könnte daher rühren, dass keine klare Vorstellung davon existiert, was eigentlich geschützt werden soll, oder zumindest nicht klar kommuniziert ist, was als Schutzgegenstand angenommen wird. Im Folgenden werden daher die künstlichen neuronalen Netze und Random Forest-Systeme - stellvertretend für eine große Zahl verschiedener ML-Modelle - analytisch in ihre Bestandteile zerlegt, mit dem Ziel, klare Schutzgegenstände zu identifizieren.

Um ML-Modelle einer im UrhG bestehenden Werkkategorie zuzuordnen, ist zunächst erforderlich, die Modelle näher zu betrachten, um anhand der identifizierten einzelnen Bestandteile eine Kategorisierung vornehmen zu können. Im Folgenden werden dazu die im Zeitpunkt der Entstehung der Arbeit überwiegend verwendeten Frameworks bzw. Technologien analysiert, Gemeinsamkeiten herausgearbeitet und im nächsten Abschnitt auf ihre urheberrechtliche Schutzfähigkeit hin untersucht.

\section{A. Grundlegende Begriffe}

Dieses Kapitel wird in die Strukturen und technischen Abläufe der Entstehung von ML-Modellen eintauchen. Dabei werden einige, in der Softwareentwicklung übliche, grundlegende Begriffe verwendet, die dem juristischen Leser möglicherweise nicht - oder nicht in diesem Kontext - geläufig sind und daher zum besseren Verständnis nachfolgend eingeführt werden. 


\section{Frameworks und Bibliotheken}

Frameworks und Bibliotheken ${ }^{208}$ sind zu verstehen als programmiersprachenspezifische Baukästen, die in ein bestehendes Programmierprojekt eingebunden werden können, und dann eine Vielzahl an Funktionalitäten bereitstellen. ${ }^{209}$ Auf diese Funktionalitäten kann der Entwickler über vordefinierte Schnittstellen bzw. Befehle zugreifen, diese sind in der Regel in sogenannten Application Programming Interfaces (APIs) dokumentiert.

\section{API}

Die Abkürzung „API“ steht für Application Programming Interface und ist die Bezeichnung für eine Schnittstelle zu einer Software-Anwendung, die üblicherweise bereitgestellt wird, um die Kompatibilität von Programmen zu ermöglichen, etwa durch Datenaustausch oder Programmerweiterungen. ${ }^{210}$ Häufig wird damit (unpräzise) auch eine Dokumentation aller in einem Framework oder einer Bibliothek vorhandenen Funktionen und Klassen bezeichnet. Die „Schnittstelle“ besteht selbst aus Programmteilen, die zum Beispiel über das Internet von anderen Programmen angesteuert werden können, oder innerhalb des ausgeführten Programms, wenn die „Fremdanwendung“ bzw. Bibliothek dort eingebunden wurde.

\section{Objekte und Funktionen}

Im Rahmen dieser Arbeit werden die Begriffe „Objekt“ und „Funktion“ häufig verwendet, weshalb an dieser Stelle ein Gefühl für die Bedeutung derselben im Softwarekontext vermittelt werden soll. Grundsätzlich ist zu

208 Die Begriffe „Framework“ und „Bibliothek“ werden in dem Kontext nicht trennscharf verwendet - so beschreibt etwa der Autor von Keras sein Projekt eher als eine „Schnittstelle“ als ein „Framework“ (vgl. https://github.com/keras-team/keras/ issues/5050\#issuecomment-272945570 (Stand: 22.02.2021)), während Wikipedia Keras als „Bibliothek“ bezeichnet (vgl. https://de.wikipedia.org/wiki/Keras (Stand: 22.02.2021)) und auf der projekteigenen Website ist die Rede vom ,,most used deep learning framework“(vgl. https://keras.io/ (Stand: 22.02.2021)). Vorliegend wird deshalb auch das Begriffspaar Framework/Bibliothek verwendet.

209 Vgl. Gamma et al., Design Patterns, S. 26.

210 Vgl. auch Fischer/Hofer, Lexikon der Informatik, S. 45. 


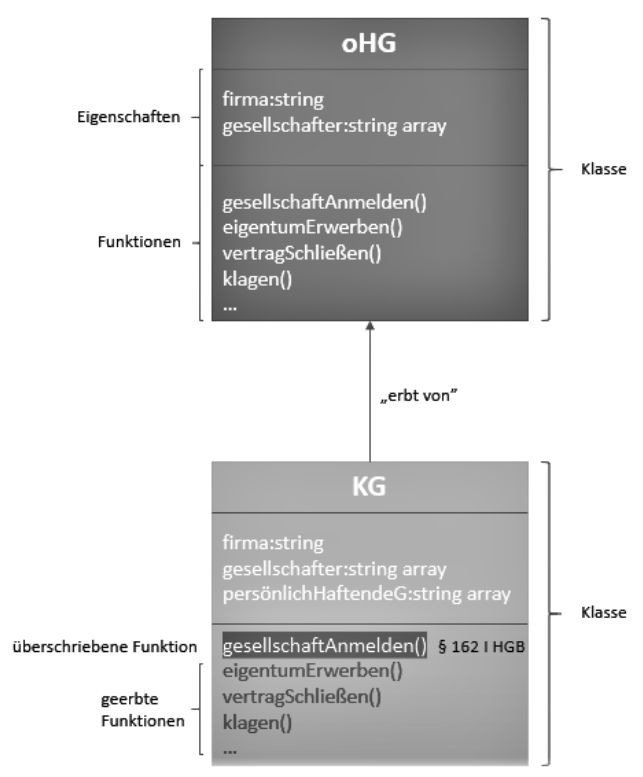

Abbildung 6.1: „Objektorientierung“ im HGB, eigene Darstellung.

unterscheiden zwischen prozeduraler und objektorientierter Programmierung (OOP). Im ML-Kontext kommt in der Regel OOP zum Einsatz. Diese lässt sich für Juristen anhand einem übertragenden Beispiel aus dem Handelsgesetzbuch erklären, vgl. dazu Abbildung 6.1 $1^{211}$ :

In $\S 105$ HGB wird die offene Handelsgesellschaft (oHG) definiert. Dabei werden ihr „Eigenschaften“ zugewiesen: Ihr Zweck ist auf den Betrieb eines Handelsgewerbes unter gemeinschaftlicher Firma gerichtet. Außerdem ist bei keinem der Gesellschafter die Haftung gegenüber den Gesellschaftsgläubigern beschränkt. Zudem hat die oHG Fähigkeiten („Funktionen“): Sie kann gem. § 124 Abs. 1 HGB unter ihrer Firma Rechte erwerben und Verbindlichkeiten eingehen. Sie kann Eigentum und andere dingliche Rechte an Grundstücken erwerben, vor Gericht klagen und verklagt werden. Das Gesetz definiert also ein abstraktes Konstrukt, das im Rahmen objektorientierter Programmierung als „Klasse“ bezeichnet würde.

211 „String“ bezeichnet den Typ der Eigenschaft „Firma“ und steht für einen Wert in Form einer Zeichenkette bzw. „Text“. Ein „Array“ bezeichnet eine Sammlung von Einzelwerten. () weisen auf eine Funktion hin. 
Eine andere „Klasse“ im HGB ist die Kommanditgesellschaft (KG). Diese wird in $\S 161$ Abs. 1 HGB definiert, sie hat ebenfalls die „Eigenschaft“, dass ihr Zweck auf den Betrieb eines Handelsgewerbes unter gemeinschaftlicher Firma gerichtet ist. Außerdem ist bei einigen Gesellschaftern die Haftung gegenüber den Gesellschaftsgläubigern auf den Betrag einer bestimmten Vermögenseinlage beschränkt, während bei einem anderen Teil der Gesellschafter eine Beschränkung der Haftung nicht stattfindet. In § 161 Abs. 2 HGB wird außerdem bestimmt, dass die Vorschriften, die für die oHG gelten, auch für die KG Anwendung finden, wenn nicht in dem Abschnitt über die KG etwas anderes bestimmt ist. In der objektorientierten Programmierung ließe sich sagen: „Die Klasse KG erbt ["Eigenschaften,, und “Funktionen,,] von der Klasse oHG“, das gilt, solange in der Klasse KG nicht „Eigenschaften“ und „Funktionen“ überschrieben werden (,überschrieben“ wird eine Eigenschaft, wenn ihr ein anderer Wert zugewiesen wird - wenn also der Gesetzgeber, um beim Beispiel zu bleiben, für die KG speziellere Vorschriften festlegt, die für die oHG nicht gelten). Wie in der Gesetzgebung werden auch in der OOP Maßnahmen zur Vereinfachung dergestalt eingesetzt, dass soweit möglich Redundanzen vermieden und Gemeinsamkeiten zentral geregelt werden ${ }^{212}$ (vgl. etwa auch den allgemeinen Teil gegenüber dem besonderen Teil einiger Gesetze).

„Klassen“ sind in der OOP-Welt wie abstrakte Schablonen, „Objekte“ hingegen sind konkrete Instanzen einer „Klasse“. ${ }^{213}$ Beide bündeln „Funktionen“ und „Eigenschaften“. ${ }^{214}$ Ein Unternehmen Müller, Meier, Schmidt oHG wäre ein Objekt (mit ausgefüllten Eigenschaften) der „Klasse“ oHG gem. § 105 HGB. Eine „Klasse“ gibt es innerhalb eines Projektes bzw. einer Bibliothek jeweils nur einmal, es kann aber unzählig viele „Objekte“ (Instanzen) dieser „Klasse“ geben.

„Funktionen“ (in Abbildung 6.1 durch runde Klammern gekennzeichnet, teilweise auch „Methoden“ oder „Operationen“ genannt ${ }^{215}$ ) kann eine Vielzahl von Programmbefehlen zugeordnet werden. So könnte die Funktion der oHG vertragSchließen () etwa die Vertragsparteien zu identifizieren haben, die essentialia negotii dokumentieren, eine Anweisung an eine Zahlungsstelle ausgeben etc.

212 Vgl. Gamma et al., Design Patterns, S. 15.

213 Vgl. Dies., Design Patterns, S. 14; „Instanz“ meint in der Softwareentwicklung einen konkreten Gegenstand vom Typ der „Klasse“, so wäre die „Karl Friedrich oHG“ eine Instanz vom Typ ,oHG“.

214 Vgl. Dies., Design Patterns, S. 11.

215 Vgl. Dies., Design Patterns, S. 11. 


\section{B. Grundbausteine für ML-Modelle: Frameworks, Bibliotheken, APIs}

Es ist heute nicht erforderlich, ein ML-Modell von Grund auf neu zu entwickeln. Den Programmierern stehen zahlreiche Frameworks und Bibliotheken zur Verfügung, die sie für die Umsetzung ihrer Konzepte nutzen können. Beispiele für solche Machine Learning-Frameworks sind für die Programmiersprache Python TensorFlow ${ }^{216}$ Keras,${ }^{217}$ PyTorch ${ }^{218}$ und ScikitLearn ${ }^{219}$ für die Sprache $R$ insbesondere das Paket randomForest ${ }^{220}$. Diese Arbeit wird sich in den folgenden Kapiteln zur Analyse der ML-Modelle stets auf diese vier Frameworks und Bibliotheken und das $R$-Paket beziehen, um praxistaugliche Ergebnisse zu erzielen.

Mit diesen Frameworks können unterschiedlichste Arten von MLModellen realisiert werden - künstliche neuronale Netze sind nur eine davon. Insbesondere können auch mit den Python-Frameworks Random Forest-Modelle erzeugt werden. Die Ausführungen mit Bezug zu den Frameworks sind in der Regel für alle Modelltypen gültig, die mit diesen Frameworks und Bibliotheken umgesetzt werden können. Es wird daher einheitlich von ML-Modellen gesprochen. Eine Abgrenzung erfolgt lediglich zu den Random Forests in $R$, da sich hier strukturelle Unterschiede zu den Modellen in Python ergeben.

In Bezug auf die Frage nach dem Schutzgegenstand sind die Frameworks und Bibliotheken jedoch der falsche Anknüpfungspunkt. Sie mögen zwar ausschnittsweise in dem Programm enthalten sein, das letztendlich in der Lage ist, die Aufgaben zu lösen, die dem ML-Modell gestellt wurden, jedoch sind sie - für sich genommen - eher mit der Palette des Malers vergleichbar, der für die Umsetzung seines Werkes daraus erst noch Farben, Intensität, Anordnung und Motiv bestimmen muss. Wenngleich der Schutz der Frameworks und Bibliotheken selbst auch diskutiert werden könnte ${ }^{221}$ ist dies jedoch nicht Ziel dieser Arbeit, und wird daher nicht thematisiert.

216 Abadi et al., TensorFlow: A system for large-scale machine learning, $265 \mathrm{ff} .$.

217 Chollet et al., Keras.

218 Paszke et al., Automatic differentiation in PyTorch.

219 Buitinck et al., API design for machine learning software: experiences from the scikit-learn project.

220 Liaw/Wiener, R News 2 Nr. 32002.

221 Vgl. z. B. Dreier/Schulze-Dreier, UrhG, § 69a Rn. 23 zum Schutz von Interfaces; Schricker/Loewenheim-Spindler, Urheberrecht, § 69a Rn. 11 zu Programmbibliotheken. 


\section{Quellcode}

Sowohl in der Vorbereitung der Trainingsdaten als Input für ML-Modelle, als auch im Training und dem Produktiveinsatz von KI spielt Quellcode - in Abgrenzung zu Parameterwerten und Hyperparametern ${ }^{222}$ - eine wesentliche Rolle. „Quellcode“ wird der vom Menschen geschriebene, menschenlesbare Programmcode genannt, der dann von speziellen Programmen ,verstanden“ werden und in Anweisungen an den Computer übersetzt werden kann. ${ }^{223} \mathrm{Er}$ ist erforderlich, um einem ML-Modell Gestalt zu verleihen, Daten einzulesen, Daten zu bearbeiten bzw. vorzubereiten, vorgefertigte Modelle einzubinden, diese mit Daten zu versehen und den Trainingsvorgang anzustoßen. Er ist quasi das „Drehbuch“ für eine Vorhersage eines Wertes oder die Erzeugung eines Bildes mittels eines ML-Modells. Er gibt die Akteure vor, ihre Gestalt, wann sie die Bühne betreten und mit wem sie interagieren. Ist ein ML-Modell trainiert, kann es mithilfe von Quellcode in die gewünschte Umgebung (etwa eine Webseite oder eine umfangreiche Analysesoftware) eingebunden und ausgeführt werden.

\section{Trainiertes Modell in Python}

Im Umgang mit ML-Modellen, die mit den beschrieben Python-Frameworks bzw. Bibliotheken erzeugt werden, ist das Ziel der Entwicklung immer ein fertiges, trainiertes ML-Modell, das schließlich zur Bilderkennung oder Bildgenerierung, Regression oder einer anderen zu Trainingsbeginn definierten Aufgabe produktiv eingesetzt werden soll. Ein trainiertes Modell stellt mitunter den Kern eines neuen Produktes dar, mit dem ein Unternehmen in den Markt einsteigen möchte. Es ist daher verständlich, dass ein großes Interesse daran besteht, das Ergebnis exklusiv verwerten und andere von der Nutzung ausschließen zu können. Fraglich erscheint jedoch, ob das Urheberrecht dies leisten kann. Wenngleich die Zahl der Konflikte bisher noch überschaubar sein mag (ein Großteil der Entwicklung von ML-Modellen bewegt sich im Open Source-Sektor, zudem wird auch noch sehr viel an der Technologie geforscht, sodass viele Entwickler bereit sind, ihre Ergebnisse freigiebig mit anderen Forschern zu teilen) so ist es doch absehbar, dass in der Zukunft durchaus Regelungsbedarf bestehen wird, insbesondere wenn Entwickler be-

222 Vgl. zur Begriffsklärung oben § 2 B.III.6..

223 Vgl. Fischer/Hofer, Lexikon der Informatik, S. 721. 
ginnen, ihre Ergebnisse nicht mehr unter sehr liberale Open Source-Lizenzen zu stellen (bisher kommen vor allem die MIT- und die BSD-Lizenz zum Einsatz). ${ }^{224}$ Aber ist eine Lizenzierung überhaupt möglich? Besteht geistiges Eigentum an einem fertig trainierten Modell? Oder können andere Rechte in Anspruch genommen werden?

Um diese Frage zu beantworten, gilt es zunächst, den Begriff „,trainiertes ML-Modell“ genau zu umreißen. Hier wird als „ML-Modell“ jede Form von Modellen ${ }^{225}$ erfasst, die unter Einsatz der genannten Frameworks und Bibliotheken mit Python erzeugt werden können. Eine Beschränkung auf künstliche neuronale Netze erfolgt nicht, weil die Ausführungen zwar auch, aber nicht ausschließlich auf künstliche neuronale Netze zutreffen. Was ein trainiertes ML-Modell technisch ausmacht, wird folgend in $\S 6$ D.II. erläutert.

In der Literatur wird hinsichtlich eines trainierten Modells bisher häufig entweder nur auf die „Trainingsergebnisse“ abgestellt ${ }^{226}$ oder implizit unter dem Begriff „trainiertes künstliches neuronales Netz“ nur die Parameter bzw. Gewichtungsinformationen berücksichtigt ${ }^{227}$. Übersehen wird dabei jedoch, dass es für die Erzielung tauglicher Ergebnisse auch und gerade auf die Hyperparameter ankommt ${ }^{228}$ und folglich diese möglicherweise in den Begriff des trainierten Modells einzubeziehen sind.

\section{Vorab: Einsatz eines trainierten Modells}

In der Praxis wird beim Einsatz eines ML-Modells für die aufgabengemäße Verwendung üblicherweise wie folgt vorgegangen, vgl. dazu auch den Teil

224 Vgl. MIT-Lizenz: https://spdx.org/licenses/MIT.html\#licenseText (Stand: 22.02.2021); BSD-Lizenz: https://spdx.org/licenses/BSD-3-Clause (Stand: 10.02.2021); ,liberal“ bedeutet in diesem Kontext, dass die Lizenzen es nicht untersagen, den Quellcode in Projekte bzw. Produkte einzubinden, die den Quellcode nicht für jedermann verfügbar offenlegen, es wird dann auch von „Non-Copyleft-Lizenzen“ gesprochen, vgl. https://ifross.org/?q=welche-lizenztypengibt-es-bei-open-source-software-und-unterscheiden-sie-sich (Stand: 22.02.2021).

225 Vgl. zum Begriff des Modells schon § 2 B.I.4..

226 Ehinger/Stiemerling, CR 12 2018, 761, 766 Rn. 45; Hauck/Cevc, ZGE 11 2019, S. $161 \mathrm{ff.}$.

227 Hartmann/Prinz, WRP 12 2018, 1431, 1437 Rn. 62.

228 Dies wird teilweise erkannt und als „Architektur“ oder „Topologie“ bezeichnet, jedoch stets separat von den Parametern gesehen und nicht als ein Ganzes betrachtet, vgl. z. B.Dies., WRP 12 2018, 1431, 1434. 


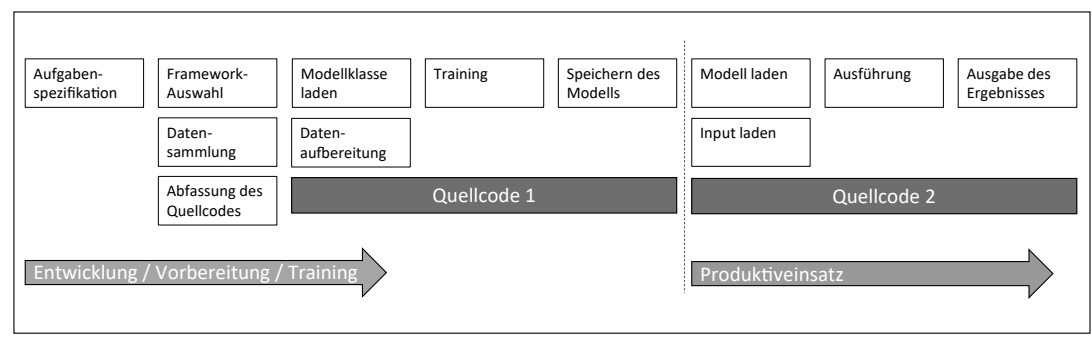

Abbildung 6.2: Entstehung eines ML-Modells, vereinfachte, eigene Darstellung.

„Produktiveinsatz“ in der Abbildung 6.2229 , nachdem das ML-Modell trainiert und gespeichert wurde: ${ }^{230}$

Es wird ein Programm (z. B. in der Programmiersprache Python) entwickelt, das - mindestens - erforderliche Bibliotheken lädt - vgl. in Abbildung 6.2 Quellcode 2.

Zudem werden - je nach dem welche Elemente des ML-Modells gespeichert vorliegen - entweder die gesamte Konfiguration des Modells, also die Hyperparameter und die Parameter, oder nur einzelne Informationen oder Informationspakete (abhängig von dem verwendeten Framework) geladen (,Modell laden“). Alternativ, wenn das gesamte zuvor trainierte ML-ModellObjekt im Binärformat gespeichert wurde, wird entsprechend das Objekt geladen (,Modell laden“). Zudem werden die Daten geladen, für die eine Vorhersage durchgeführt werden soll (,Input laden“).

Anschließend wird die Vorhersagefunktion (z. B. predict ( ) ) aufgerufen, um das konkrete Problem oder die konkrete Aufgabe (etwa die Objekterkennung in einem Bild) zu lösen („Ausführung“). Erst nach der Ausführung dieses Codes wird die Lösung der Aufgabe oder des Problems ausgegeben.

229 Abbildung 6.2 soll nur dazu dienen, wesentliche Schritte in der Modellentstehung visuell nachzuverfolgen. Vernachlässigt werden dabei u. a. Prozessschritte iterativer Überarbeitung und Anpassung sowie Test- und Evaluierungsphasen.

$230 \mathrm{Vgl}$. z. B. für ein Minimalbeispiel in Scikit-Learn, das den hier dargestellten Ablauf in Code umsetzt: https://scikit-learn.org/dev/modules/model_persistence.html\# python-specific-serialization (Stand: 22.02.2021). 


\section{Begriff des trainierten Modells}

Für die konkrete Problemlösung kommt es also maßgeblich darauf an, was am Ende der Ausführung von Quellcode 1 abgespeichert wurde, denn das Abgespeicherte wird dann in Quellcode 2 geladen („Modell laden“). Andernfalls müsste das Modell für jeden Einsatz vollständig neu trainiert werden. Je nach verwendeter Programmiersprache und Framework stehen dem Entwickler zum Abspeichern des Modells und seiner Bestandteile unterschiedliche Möglichkeiten zur Verfügung. Die Technologie wird ständig weiterentwickelt, eine Betrachtung der Möglichkeiten kann folglich nur eine Momentaufnahme darstellen.

Dennoch ist es der urheberrechtlichen Bewertung zuträglich, sich mit den bisherigen Möglichkeiten auseinanderzusetzen, um ein Grundverständnis für die relevanten Vorgänge zu entwickeln. Es folgt daher eine Übersicht der Speichervarianten jeweils in TensorFlow, Keras, PyTorch und Scikit-Learn. Identifiziert werden soll dabei, in welcher Form die Ergebnisse gespeichert und dementsprechend auch wieder geladen - werden, um anschließend den passenden urheberrechtlichen Schutz ermitteln zu können. Es werden nur Lösungen basierend auf der Programmiersprache Python thematisiert, Ziel ist es aber, am Ende dieses Kapitels auch auf andere Programmiersprachen übertragbare Grundsätze zu entwickeln.

\section{1. „Trainiertes Modell“ in TensorFlow}

TensorFlow ist eine „End-to-End Open Source Machine Learning Platform". ${ }^{231}$ Es handelt sich um eine Software-Bibliothek, die seit November $2015^{232}$ von Google entwickelt wird und unter der Open Source-Lizenz Apache License 2.0 für die Entwicklung von Machine Learning-Projekten zur Verfügung steht. Die Plattform basiert auf der Programmiersprache Python und ermöglicht es, mittels vorgefertigter Klassen und Funktionen, ML-Projekte zügig umzusetzen.

TensorFlow bietet verschiedene Möglichkeiten, die durch das Training erarbeiteten Fortschritte des Modells zu sichern. Zum einen speichert TensorFlow während des Trainings immer wieder sogenannte Checkpoint-Dateien. Das

231 https://www.tensorflow.org (Stand: 22.02.2021).

232 Geht hervor aus der Versionsgeschichte auf GitHub, https://github.com/tensorflow/ tensorflow/commits/master/RELEASE.md (Stand: 22.02.2021). 
sind Dateien im für Menschen nicht lesbaren Binärformat, die ein Verzeichnis berechneter Tensoren, ${ }^{233}$ also Parameter bzw. Gewichtungsinformationen, beinhalten. ${ }^{234}$

Mit jedem Trainingsvorgang wird die aktuellste Checkpoint-Datei geladen und das Modell damit initialisiert, sodass eine Fortsetzung des Trainings nach einer Unterbrechung möglich ist und nicht von vorne begonnen werden muss. $^{235}$

Ist das Training abgeschlossen, kann das Modell zum Beispiel mittels der Funktion $t f$.saved_model.save () exportiert und im Anschluss mittels tf.saved_model.load () wieder eingelesen werden. ${ }^{236}$ Das hat zur Folge, dass eine sogenannte ProtocolBuffer-Datei (saved_model.pb) und zwei Verzeichnisse namens ,assets“ und „variables“ erzeugt werden. ${ }^{237}$ Das Verzeichnis „,variables“ enthält einen Training-Checkpoint, ,assets“ enthält zusätzliche Informationen (zum Beispiel Textdateien für Vokabeltabellen, falls textverarbeitende Modelle entwickelt werden). ${ }^{238}$ TensorFlow stellt auBerdem (noch, dies ist eine Funktion aus der TensorFlow Version 1) die Funktionalität Saver. save () bereit, mit der bei entsprechender Einstellung vier Dateien erzeugt werden können - eine enthält dann die Parameter (.data), eine zweite weitere Informationen zum Checkpoint-Index (.index), eine dritte eine Liste aller zu speichernden Checkpoints (.pb) und eine die Graph-Struktur (.meta). ${ }^{239}$

Fraglich ist also, welche dieser bereitgestellten Funktionalitäten dem Schutzgegenstand ,trainiertes ML-Modell“im Sinne dieser Arbeit entspricht. Die Checkpoints enthalten keine Informationen über die Struktur des Modells, sind also nicht dazu einsetzbar, das Modell ohne anderweitige Informationen zu laden. Dazu sind hingegen grundsätzlich die Ergebnisse von Saver.save () und tf.saved_model.save () geeignet. Diese Arbeit stellt daher im weiteren Verlauf auf die Ergebnisse der Aufrufe von tf.saved_model.save () sowie Saver.save () ab, wenn es um ein „trainiertes Modell“" in TensorFlow geht.

233 Zum Begriff vgl. Fußnote 121; eine Einführung in Tensoren gibt auch TensorFlow: https://www.tensorflow.org/guide/tensor (Stand: 22.02.2021).

234 https://www.tensorflow.org/guide/checkpoint (Stand: 22.02.2021).

235 Initialisierung: Setzen von Anfangswerten bei Start eines Programmes, vgl. auch https://www.dwds.de/wb/Initialisierung (Stand: 22.02.2021).

236 Vgl. https://www.tensorflow.org/guide/saved_model (Stand: 22.02.2021).

237 Vgl. https://www.tensorflow.org/guide/saved_model (Stand: 22.02.2021).

238 Vgl. https://www.tensorflow.org/guide/saved_model (Stand: 22.02.2021).

239 Vgl. https://www.tensorflow.org/api_docs/python/tf/compat/v1/train/Saver (Stand: 22.02.2021). 


\section{2. „Trainiertes Modell“ in Keras}

Auf der Projekt-Website wird Keras beschrieben als „The Python Deep Learning Library“, bzw. als „High-Level Neural Network API“, die auf TensorFlow aufsetzt. ${ }^{240}$ Keras stellt diverse Möglichkeiten bereit, ein trainiertes Modell zu exportieren bzw. zu speichern. ${ }^{241}$

- model . save (<Dateipfad $>$ ) Erzeugt eine Datei im binären HDF5Format (*.h5), die die Architektur des Modells beinhaltet sowie die berechneten Gewichte, die Trainingskonfiguration (etwa die Verlustund Optimierungsfunktion $)^{242}$ sowie den Zustand des Optimierers ${ }^{243}$ im Zeitpunkt des Abspeicherns. ${ }^{244}$ Mithilfe dieser Datei kann das Training dort fortgesetzt werden, wo es unterbrochen wurde. Alternativ kann auch das SavedModel-Format von TensorFlow verwendet werden.

- model.to_json() / model.to_yaml() Speichert nur die Architektur des Modells in einem menschenlesbaren Format (nicht binär, sondern strukturierte Textdaten entweder im JSON- oder YAMLFormat). ${ }^{245}$

- model.save_weights () Erzeugt eine .h5-Datei, die nur die Gewichtsinformationen enthält. ${ }^{246}$ Diese können dann in ein Modell geladen werden. Mithilfe der Layer-Namen können Inhalte - Gewichte - selektiert werden.

- keras.callbacks.ModelCheckpoint (...) Damit kann das Modell z. B. nach jeder Epoche gespeichert werden - die Funktion ruft dann entweder model.save_weights() oder model.save() auf. ${ }^{247}$

240 Vgl. https://keras.io (Stand: 22.02.2021).

241 Vgl. TensorFlow-Keras-Dokumentation, https://www.tensorflow.org/guide/keras/ save_and_serialize?hl=en (Stand: 22.02.2021), bzw. Keras-API-Dokumentation, https://keras.io/api/models/model_saving_apis/ (Stand: 22.02.2021).

242 Funktionen, die errechnen, wie weit das Modell vom gewünschten Zielwert - vorgegeben z. B. durch die Labels der Trainingsdaten - entfernt ist, vgl. auch § 2 B.III.7..

243 Der „Optimierer“ ist eine Funktionalität, die zwischen Verlustfunktion und Modell eine Rückkopplung herstellt und die Parameter des Modells entsprechend anpasst.

244 Vgl. Keras-Dokumentation, https://keras.io/api/models/model_saving_apis/\#savemethod (Stand: 22.02.2021).

245 Vgl. Keras-Dokumentation, https://keras.io/api/models/model_saving_apis/\#tojsonmethod (Stand: 22.02.2021).

246 Vgl. https://keras.io/api/models/model_saving_apis/\#saveweights-method (Stand: 22.02.2021).

247 Vgl. TensorFlow-Keras-Dokumentation, https://www.tensorflow.org/api_docs/ python/tf/keras/callbacks/ModelCheckpoint (Stand: 22.02.2021). 
- keras.utils.plot_model (model, ....) Konvertiert ein $\mathrm{Ke}$ ras-Modell in ein Format, das eine grafische Ausgabe der Architektur bzw. der Hyperparameter des Modells erzeugt. ${ }^{248}$

Dem „trainierten Modell“ im Sinne dieser Arbeit entspricht in Keras das Ergebnis von model.save (<Dateipfad $>$ ), da hier alle Informationen, die zur Ausführung des Modells erforderlich sind, abgelegt werden.

\section{3. „Trainiertes Modell“ in PyTorch}

Auf der PyTorch-Website wird PyTorch beschrieben als ein „Open Source Machine Learning Framework, das den Weg vom Research Prototyping zum Production Deployment beschleunigt“ ${ }^{249}$ also von der Entwicklung bis zum Einsatz des fertig trainierten Modells in der Zielumgebung verwendet werden kann.

Auch in PyTorch gibt es unterschiedliche Möglichkeiten, Trainingsergebnisse bzw. ML-Modelle zu speichern:

- torch.save(the_model.state_dict(), PATH) speichert und lädt nur die Modellparameter. ${ }^{250}$

- torch.save (the_model, PATH) speichert und lädt das gesamte Modell als ein Objekt, dabei wird ein Tool verwendet, das sich „Pickle“ nennt. ${ }^{251}$

248 Vgl. TensorFlow-Keras-Dokumentation, https://www.tensorflow.org/api_docs/ python/tf/keras/utils/plot_model (Stand: 22.02.2021).

249 https://pytorch.org/ (Stand: 22.02.2021)

250 PyTorch-Dokumentation, https://pytorch.org/tutorials/beginner/saving_loading_ models.html (Stand: 22.02.2021).

251 Das Pickle-Modul in Python implementiert binäre Protokolle für die Serialisierung und Deserialisierung von Python-Objektstrukturen. „Pickling“ ist der Vorgang durch den eine Python-Objekthierarchie in einen Byte-Stream umgewandelt wird, und „unpickling“ ist der umgekehrte Vorgang, bei dem ein Byte-Stream zurück in eine Objekthierarchie gewandelt wird. Der Dateiinhalt ist nicht „,menschenlesbar“, sondern nur für das Laden in ein Programm vorgesehen; https: //docs.python.org/3/library/pickle.html (Stand: 22.02.2021). 
- torch.nn.Module.load_state_dict () Lädt die Parameter eines Modells in Form eines Dictionaries ${ }^{252}$ unter Verwendung eines deserialisierten (also ,unpickled“) state_dict-Objektes. ${ }^{253}$

Ein state_dict ist ein Python Dictionary-Objekt, das die Beziehungen jeder Schicht des Modells zu ihrem Parameter-Tensor enthält.

Optimiererobjekte haben ebenfalls ein state_dict, das Informationen über den Zustand des Optimierers enthält, sowie die verwendeten Hyperparameter. $^{254}$

Das Ergebnis eines Aufrufs von torch.save (the_model, PATH) entspricht dem, was hier als ,trainiertes Modell“" verstanden wird, denn die anderen beiden Funktionen speichern im Gegensatz dazu nur einzelne Bestandteile des Modells und sind im Ergebnis nicht ausreichend, um das Modell ohne weitere Informationen über die Architektur wiederherzustellen.

\section{4. „Trainiertes Modell“ in Scikit-Learn}

Scikit-Learn stellt simple und effiziente Tools für Data Mining und Datenanalyse zur Verfügung, die frei zugänglich und unter der Open Source BSD-Lizenz verfügbar sind. ${ }^{255}$ Bezüglich der Speicherung eines Modells verweist die Dokumentation von Scikit-Learn zum einen auf die PickleFunktionalität von Python, und erwähnt zum anderen die Möglichkeit, das erstellte und trainierte Modell in andere Formate zu exportieren. ${ }^{256}$ Aus der exportierten Form kann das Modell nicht weiter trainiert, sondern „nur noch“ für Vorhersagen - also den Produktiveinsatz - verwendet werden. ${ }^{257}$

252 Ein Dictionary-Objekt ist ein Objekt, das wie ein Nachschlagewerk funktioniert: Unter Schlüsselwörtern (keys) sind Informationen abrufbar

253 PyTorch-Dokumentation, https://pytorch.org/tutorials/beginner/saving_loading_ models.html\#save-load-state-dict-recommended (Stand: 22.02.2021).

254 Output der state_dict-Datei, insb. des Optimizer state dict einsehbar auf der Website https://pytorch.org/tutorials/beginner/saving_loading_models.html, zuletzt abgerufen am 22.02.2021.

255 Vgl. Scikit-Learn-Dokumentation, https://scikit-learn.org/stable/index.html (Stand: 22.02.2021).

256 Vgl. Scikit-Learn-Dokumentation, https://scikit-learn.org/stable/modules/model_ persistence.html (Stand: 22.02.2021).

257 Vgl. Scikit-Learn-Dokumentation, https://scikit-learn.org/stable/modules/model_ persistence.html (Stand: 22.02.2021). 
Die Autoren von Scikit-Learn beschreiben den Prozess, ein mit zukünftigen Versionen des Frameworks zuverlässig wiederherstellbares Modell zu speichern: Es ist nicht nur das Modell zu speichern, sondern auch die Metadaten (hier: die Trainingsdaten, der Python-Quellcode mit dem das Modell erstellt wurde, die Scikit-Learn-Version und eventuelle Abhängigkeiten zu anderen Tools, sowie der Kreuzvalidierungswert der mit den Trainingsdaten erzielt wurde, damit geprüft werden kann, ob das Modell erfolgreich wiederhergestellt wurde). ${ }^{258}$

Das Speichern eines ,trainierten Modells“ im Sinne dieser Arbeit erfolgt also wie schon in PyTorch mithilfe des Pickle-Moduls, eine Scikit-Learnspezifische Methode muss dafür nicht zum Einsatz kommen, sondern es werden die „Bordmittel“ von Python eingesetzt.

\section{Zusammenfassung und Definition}

Ziel des Trainings eines ML-Modells ist es, ein Softwaregebilde zu schaffen, das reproduzierbar und zuverlässig für den gleichen Input die gleichen Vorhersagen bzw. Ergebnisse (Output) liefert. Dies kann nur erreicht werden, indem die während des Trainings noch manipulierbare Struktur des Modells am Ende des Trainings eingefroren und wiederabrufbar gemacht wird. Dazu unerlässlich sind

- Hyperparameter,

- Parameter, und

- Quellcode.

Die Hyperparameter sind erforderlich, weil sie das „Gerüst“ des Modells beschreiben. Die Parameter sind erforderlich, weil sie das Gerüst des Modells mit Werten füllen. Der passende Quellcode führt alles zusammen.

Ein trainiertes ML-Modell liegt also vor, wenn eine Dateistruktur existiert, die von einem Quellcode oder Script verwendet wird, wobei das daraus entstehende Modellobjekt ohne weitere Zwischenschritte zur bei Entwick-

258 Vgl. Scikit-Learn-Dokumentation, https://scikit-learn.org/stable/modules/model_ persistence.html (Stand: 22.02.2021). 


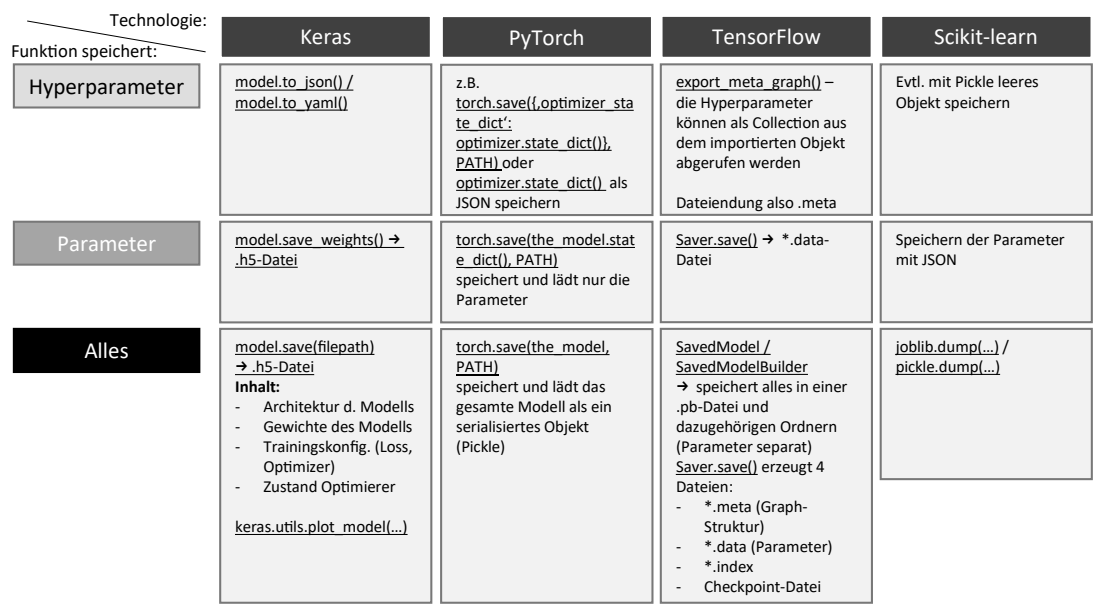

Abbildung 6.3: Überblick über Speichervarianten in den untersuchten Frameworks, eigene Darstellung.

lung des Modells angestrebten Problemlösung eingesetzt werden kann. ${ }^{259}$ Grundsätzlich ist also immer ein aufrufender Quellcode bzw. ein aufrufendes Skript erforderlich, sowie eine oder mehrere Dateien, die Informationen über das Modell enthalten. Der Grad zwischen Quellcode bzw. Skript und Datei, auf dem die Informationen verteilt sind, ist je nach verwendetem Framework unterschiedlich. Für einen abschließenden Überblick vgl. Abbildung 6.3.

\section{E. Trainierter Random Forest in $\mathrm{R}$}

Da sich für ein Random Forest-Modell, das nicht in Python, sondern in $R$ konzipiert und trainiert wurde, ${ }^{260}$ Unterschiede im Vergleich zur Implementierung in Python ergeben, erfolgt hier eine differenzierte Darstellung.

259 Theoretisch wäre es auch denkbar, ein trainiertes Modell zu erzeugen, das auch ohne Ablage in Dateien im Arbeitsspeicher eines Rechners mit endloser Runtime für immer existiert. Ein solches, doch eher fernliegendes, Konstrukt soll jedoch hier von der Definition nicht erfasst sein.

260 Die Verwendung der Programmiersprache $R$ scheint für Random Forests nahezu so verbreitet wie Python, und soll deshalb hier auch Berücksichtigung finden. 
Ein trainiertes Random Forest-Modell enthält eine Vielzahl von ungleichen Entscheidungsbäumen. ${ }^{261}$ Jeder Entscheidungsbaum besteht aus einer Vielzahl an Knoten, die jeweils ein Feature hinsichtlich eines Schwellwertes überprüfen. „Trainiert“ werden die Schwellwerte, anhand derer entschieden wird, ob ein Datensatz zum linken oder rechten Tochterknoten weitergeleitet wird. Im Ergebnis entsteht eine als Tabelle abrufbare Datensammlung, die - in der Implementierung des Pakets ,,randomForest“ in der Programmiersprache $R$ - für jeden konstruierten Baum in sechs Spalten die Baumstruktur enthält ${ }^{262}$.

Folgende sechs Spalten entstehen dabei:

- Linker Tochterknoten

- Rechter Tochterknoten

- Gewählte Split-Variable bzw. - Vorhersage (nur für Endknoten Feature

- Split-Point bzw. Schwellenwert
- Status: handelt es sich um einen Endknoten? relevant)

Diese Spalten enthalten alle Informationen, die zur Durchführung der Vorhersagen für neue Daten erforderlich sind. Für den Produktiveinsatz eines Random Forest in $R$ ist folglich der Zugriff auf die so entstandene Tabelle ausreichend, diese stellt für den weiteren Verlauf der Prüfung der Schutzmöglichkeiten von mit $R$ entwickelten Random Forest-Modellen den Schutzgegenstand dar.

\section{F. Trainierte Parameter}

Ein zentrales Element von ML-Modellen sind die Werte, die vielfach „Gewichte“, „Gewichtungsinformationen“ oder „Trainingsergebnisse“ genannt werden. In den einschlägigen Frameworkbeschreibungen ist hingegen ne-

261 Für eine ausführlichere Erklärung vgl. § 2 B.II.1..

262 Liaw/Wiener, R News 2 Nr. 3 2002, S. 4. 
ben „Weights“ ${ }^{\text {“263 }}$ auch von Parametern, ${ }^{264}$ bzw. trainierbaren Parametern ${ }^{265}$ die Rede. Diese werden stets kontrastiert mit „Hyperparametern“, 266 oder auch „System-“ oder „Metaparametern“. ${ }^{267}$ Es handelt sich um Werte, die sich in einem Modell-Optimierungsprozess (,Training“) nicht (automatisch) verändern. ${ }^{268}$

Trainierte Parameter, die aus dem Training eines Modells mit sorgfältig ausgewählten Trainingsdaten und einer entsprechenden Architektur hervorgehen, füllen das Modell mit Inhalt. Ohne die richtigen Parameter kann ein Modell nicht die richtigen Vorhersagen treffen. Es liegt daher nahe, auch die Sammlung der Parameterwerte als Schutzgegenstand ins Auge zu fassen.

\section{G. Hyperparameter}

Als Schutzgegenstand diskutiert werden auch die Hyperparameter, auch „To-

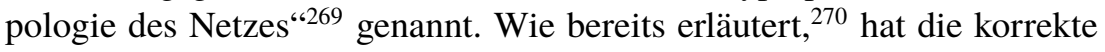
Auswahl der Anzahl zu verwendender Schichten, der Menge Neuronen je Schicht, verwendeter Funktionen und Rückkopplungen innerhalb der Struktur einen wesentlichen Einfluss darauf, wie gut das KNN gestellte Aufgaben lösen kann. ${ }^{271}$ Hyperparameter sind außerdem nicht nur für KNNs relevant, sondern für verschiedenste ML-Modelle - auch die Struktur von Random Forests wird unter anderem die maximale Baumtiefe, die Anzahl ausgewerteter Features etc. durch Hyperparameter vorgegeben. Eine sorgfältige Auswahl der passendsten Hyperparameter verleiht einem Modell die Struktur, die es braucht, um taugliche Ergebnisse zu produzieren. Die Hyperparame-

263 Weights: Keras, https://keras.io/api/models/model_saving_apis/ (Stand: 22.02.2021), TensorFlow, https://www.tensorflow.org/guide/saved_model?hl=en (Stand: 22.02.2021).

264 TensorFlow, https://www.tensorflow.org/guide/checkpoint?hl=en (Stand: 22.02.2021), PyTorch https://pytorch.org/tutorials/beginner/saving_loading_ models.html (Stand: 22.02.2021).

265 PyTorch, https://pytorch.org/tutorials/beginner/saving_loading_models.html\#whatis-a-state-dict (Stand: 22.02.2021).

266 Vgl. z. B. Osinga, Deep Learning Cookbook, S. 21; Nielsen, Neural Networks and Deep Learning, Kap. 1.

267 Ertel, Grundkurs Künstliche Intelligenz: Eine praxisorientierte Einführung, S. 304.

268 Vgl. zur Begriffsklärung auch schon oben $§ 2$ B.III.6..

269 Hartmann/Prinz, WRP 12 2018, 1431, 1434.

270 S. oben $\S 2$ B.II.2.

271 Vgl. auch Nielsen, Neural Networks and Deep Learning, Kapitel „Implementing our network to classify digits". 
ter werden mitunter immer weiter angepasst, sodass sie das Ergebnis eines langwierigen Entwicklungsprozesses darstellen können. Dementsprechend könnten auch die vom Entwickler gewählten Hyperparameter urheberrechtlichen Schutzes bedürfen, und sind im weiteren Verlauf als eigenständiger Schutzgegenstand aufzufassen.

\section{H. Zusammenfassung}

Ziel dieses Kapitels war es, Klarheit zu schaffen in Bezug auf die Bestandteile von ML-Modellen, um unmissverständliche Schutzgegenstände zu identifizieren, die im nächsten Kapitel auf ihre Schutzfähigkeit untersucht werden können. Dafür wurden zunächst die Grundbausteine für ML-Modelle identifiziert, für die sich keine (urheberrechtlichen) Besonderheiten gegenüber den Grundbausteinen anderer Software ergeben.

Sodann wurde der Begriff des ,trainierten Modells“ untersucht, und was darunter je nach eingesetzter Technologie zu verstehen ist. Aus den Erkenntnissen wurde eine die Gemeinsamkeiten der untersuchten Technologien hervorhebende allgemeine Definition für den Begriff des „trainierten Modells“ aufgestellt: Als „trainiertes Modell“ versteht diese Arbeit eine Dateistruktur (also permanent gespeicherte Informationen), die von einem Quellcode (Quellcode 2 in Abbildung 6.2) verwendet wird, wobei das daraus entstehende Modellobjekt ohne weitere Zwischenschritte zur bei Entwicklung des Modells angestrebten Problemlösung eingesetzt werden kann. Neben dem Objekt ,trainiertes Modell“ wurden zudem die einzelnen Bestandteile Quellcode, Hyperparameter und Parameter der Modelle als mögliche unabhängige Schutzgegenstände identifiziert. Für in der Sprache $R$ entwickelte Random Forests bzw. Entscheidungsbaummodelle ist wesentlicher Schutzgegenstand die durch das Training ermittelte bzw. ermittelbare Tabelle. 


\section{§ 7 Schutzmöglichkeiten im Urheberrecht und den verwandten Schutzrechten}

\section{A. Einleitung}

\section{Forschungsstand}

Bisher wird Schutz für ML-Modelle insbesondere unter dem Dach des Computerprogrammschutzes diskutiert, ${ }^{272}$ teilweise wird dies auch strikt abgelehnt ${ }^{273}$ oder es erfolgt eine differenzierte ${ }^{274}$ Betrachtung. Andere diskutieren zwar eine Einordnung als Datenbank (insbesondere im Lichte des Leistungsschutzrechtes), kommen hier jedoch entweder zu keinem einen Schutz bejahenden Ergebnis, ${ }^{275}$ oder wagen den Versuch einer Subsumtion erst gar nicht ${ }^{276}$. Wieder andere ziehen sogar den Tonträgerschutz als Möglichkeit heran, Modellen doch noch zu einem Schutz zu verhelfen, wenngleich unklar ist, wie dies in der Praxis auszusehen hätte. ${ }^{277}$

\section{Hier gewählter Lösungsansatz}

An dieser Stelle erfolgt daher unter Berücksichtigung der zuvor dargestellten technischen Gegebenheiten ein Perspektivwechsel, der Klarheit hinsichtlich der Schutzmöglichkeiten schaffen soll. Berücksichtigt wird neben den im

272 Iglesias Portela/Shamuilia/Anderberg, Intellectual Property and Artificial Intelligence: A Literature Review: EUR 30017 EN, S. 9; Linke, GRUR Junge Wissenschaft 2019, S. 47.

273 Sehr verallgemeinernd z. B. Wandtke/Bullinger-Grützmacher, PK UrhR, § 69a Rn. 21; KI auf Algorithmen reduzierend Hauck/Cevc, ZGE 11 2019, 135, 159; nur sehr eingeschränkt den Datenbankschutz für möglich haltend Apel/Kaulartz, RDi Nr.1 2020, 24, $28 \mathrm{f}$..

274 Gomille, JZ Nr. 20 2019, 969, 970; Ehinger/Stiemerling, CR 12 2018, 761, 765 Rn. 41; Hartmann/Prinz, WRP 12 2018, 1431, 1436; BT-Drs. 19/23700 S. 69 (Bericht der Enquete-Kommission KI).

275 Hauck/Cevc, ZGE 11 2019, 135, 161 f.; Hartmann/Prinz, WRP 12 2018, 1431, 1437; Loewenheim-Leistner/Zurth, Handbuch Urheberrecht, § 49 Rn. 146; Haberstumpf, GRUR 2003, 14, 19.

276 Kaulartz/Braegelmann, Rechtshandbuch Artificial Intelligence, Kap. 7.1 Rn. 24 ff..

277 Nägele/Apel in Dies., Rechtshandbuch Artificial Intelligence, Kap. 7.1 Rn. 50. 
Urheberrecht infrage kommenden Vorschriften über den Schutz als Datenbankwerk ( 4 Abs. 2 UrhG), als Computerprogramm (§ 69a UrhG) und als sonstiges Werk ( $\$ 2$ UrhG) auch ein Schutz durch das Datenbankherstellerrecht ( $\S 87 \mathrm{a}$ ff. UrhG). Ermittelt wird ein Schutz für die folgenden zuvor erläuterten Schutzgegenstände: trainiertes ML-Modell in Python (insb. trainiertes $\mathrm{KNN}$ ), trainierter Random Forest in $R$, trainierte Parameter, Hyperparameter, sowie das untrainierte Modell (in Kombination aus Hyperparametern und Parametern). Dabei wird jeder Schutzgegenstand für sich genommen anhand der infrage kommenden Vorschriften geprüft, und zwar das trainierte Modell in Python in $\S 7$ B., der trainierte Random Forest in $\S 7$ C., die trainierten Parameter in $\S 7$ D., die Hyperparameter in $\S 7$ E. und das untrainierte Modell in $\S 7$ F..

\section{B. Trainiertes ML-Modell in Python (insb. trainiertes KNN)}

Aus den vorangegangenen Erläuterungen ${ }^{278}$ geht hervor, dass der Entwickler eines ML-Modells entscheiden kann, was am Ende des Trainingsvorgangs in einer Datei abgelegt wird. In einem nächsten Schritt gilt es nun, herauszufinden, ob die so abgelegten Modellbestandteile einem urheberrechtlichen Schutz zugänglich sind.

\section{Schutz als Datenbankwerk gem. § 4 Abs. 2 UrhG}

Um einen urheberrechtlichen Schutz im Sinne von § 4 Abs. 2 UrhG zu genießen, müsste eine Datenbank folgende Voraussetzungen erfüllen: Erforderlich ist ein Datenbankwerk in Form einer Sammlung voneinander unabhängiger Elemente, die systematisch oder methodisch angeordnet und einzeln mit Hilfe elektronischer Mittel oder auf andere Weise zugänglich sind. Ferner muss die Sammlung aufgrund der Auswahl oder Anordnung eine persönliche geistige Schöpfung darstellen.

278 S. oben $§ 6$ D.. 


\section{Datenbankwerk}

Gemäß der Legaldefinition in $\S 4$ Abs. 2 UrhG ist ein Datenbankwerk ein Sammelwerk, dessen Elemente systematisch oder methodisch angeordnet und einzeln mit Hilfe elektronischer Mittel oder auf andere Weise zugänglich sind. Sammelwerk wiederum ist eine Sammlung von Werken, Daten oder anderen unabhängigen Elementen, die aufgrund der Auswahl oder Anordnung der Elemente eine persönliche geistige Schöpfung darstellen (§ 4 Abs. 1 UrhG). Zunächst ist also zu klären, ob ein trainiertes Netz die strukturellen Anforderungen an ein Datenbankwerk grundsätzlich erfüllt. Die Betrachtung hinsichtlich der Werkqualität des trainierten Netzes erfolgt in einem zweiten Schritt.

\section{a) Sammlung}

Ein trainiertes KNN müsste folglich eine Sammlung von Elementen darstellen. Elemente können gem. § 4 Abs. 1 UrhG Werke im Sinne von $§ 2$ Abs. 2 UrhG, aber auch Daten und andere unabhängige Elemente sein. ${ }^{279}$ Eine Sammlung ist eine Zusammenstellung mehrerer solcher Elemente. Den Elementen einer Sammlung muss kein Werkcharakter anhaften, es genügt, dass sie lediglich informationellen Wert aufweisen. ${ }^{280}$

Analog zum Vorgehen bei den eingangs präsentierten vier Frameworks wird erneut eine Untersuchung hinsichtlich der für die Sammlung infrage kommenden Elemente vorgenommen.

\section{aa) TensorFlow}

In TensorFlow könnte zum einen auf die durch jeden Aufruf von $\mathrm{Sa}-$ ver.save () erzeugten vier Dateien abgestellt werden. ${ }^{281}$ Es wird mithin nicht darauf rekurriert, dass - bzw. ob - ein einzelner Parameter bzw. eine einzelne „Gewichtungsinformation“ als Elemente anzusehen sind, sondern ob die genannten Dateien insgesamt Elemente einer Sammlung darstellen.

279 Dreier/Schulze-Dreier, UrhG, § 4 Rn. 9; Wandtke/Bullinger-Marquardt, PK UrhR, $\S 4$ Rn. 4.

280 Dreier/Schulze-Dreier, UrhG, § 4 Rn. 9; Wandtke/Bullinger-Marquardt, PK UrhR, § 4 Rn. 4.

281 Vgl. Ausführungen in $§ 6$ D.II.1.. 
In TensorFlow gibt es darüber hinaus die Möglichkeit, mit der SavedModel-Variante alle Informationen gesammelt in einer Protocol Buffer-Datei zu speichern $\left(* . p b / *\right.$.pbtxt). ${ }^{282}$ In beiden Fällen wären die Elemente jeweils zumindest die Graph-Struktur (also Hyperparameter) und die Parameter (Gewichtungsinformationen), für Variante 1 kommen noch der Checkpoint-Index sowie eine Liste der erstellten Checkpoints hinzu, die Sammlungen bestünden entweder in der durch Saver. save () erzeugten Zusammenstellung oder aber der Protocol Buffer-Datei.

\section{bb) Keras}

Der Sammlung entspricht in Keras die mit model. save (Dateipfad) erstellte .h5-Datei. In der Datei liegen sämtliche Informationen vor, die dazu benötigt werden, das trainierte Netz wiederherzustellen. Elemente der Sammlung sind die Architektur des Modells (die dazugehörigen Hyperparameter), die Parameter (die „Gewichte“), die Trainingskonfiguration sowie der Zustand des Optimierers. ${ }^{283}$ Auch hier wird nicht auf die einzelnen Gewichtsinformationen als Elemente abgestellt, sondern auf deren Sammlung in der .h5-Datei.

\section{cc) PyTorch}

PyTorch stellt mit torch. save (...) eine Funktion bereit, mit der das ganze KNN-Modell in serialisierter Form in einer Datei abgelegt wird. Dabei wird das Python-Tool „Pickle“284 eingesetzt. Dies hat allerdings auch zur Folge, dass etwa die Modellklasse ${ }^{285}$ nicht ,gepicklet“ wird, sondern nur eine Referenz zu der Datei, in der die Klasse definiert ist. Diese darf nicht verändert werden, wenn das Modell erfolgreich wieder geladen werden soll. Die Elemente entsprechen auch hier wieder den verschiedenen Hyperparametern und Parametern, und der Untersuchungsgegenstand „Sammlung“ ist

282 S. oben $\S 6$ D.II.1..

283 Vgl. § 6 D.II.2..

284 Name abgeleitet aus dem Englischen to pickle - konservieren / einlegen, vgl. ,pickle“, Merriam-Webster.com, 2019, https://www.merriam-webster.com, zuletzt abgerufen am 22.02.2021.

285 Also die abstrakte Definition der Modellart - vgl. zur Erklärung des Begriffs der „Klasse“ $§ 6$ A.III.. 
die durch Ausführung der Funktion torch. save (. . . ) bewirkte Zusammenstellung dieser Elemente.

\section{dd) Scikit-Learn}

Unter Zuhilfenahme von joblib.dump (...) oder pick le.dump (...) kann das gesamte mit Scikit-Learn erstellte Modell gespeichert werden. Das Modell - in Scikit-Learn repräsentiert als ein einzelnes Objekt - enthält in den ihm zugewiesenen Eigenschaften die Hyperparameter und Parameter, mithin die Elemente des trainierten KNN als Datensammlung.

\section{ee) Zusammenfassung und Subsumtion}

Entgegen der andernorts vorgenommenen Einschränkung auf die Gewichtungsinformationen eines $\mathrm{KNN}^{286}$ werden diese hier explizit nur als ein Element der Sammlung verstanden. Die Sammlung „trainiertes Modell“ besteht vielmehr aus den folgenden Elementen:

- Architektur des Modells (repräsentiert durch eine Kombination von Hyperparametern - Anzahl Schichten, Anzahl Neuronen je Schicht etc.),

- sonstige Hyperparameter (etwa die Aktivierungsfunktionen)

- sowie Parameter (als ein Element).

Fraglich erscheint, ob auch der Quellcode oder ein Skript zur Initialisierung des Netzes als Bestandteil der Sammlung eingeordnet werden müsste. Dieser ist jedoch erforderlich, um auf die Daten überhaupt zugreifen zu können. Ihn als Bestandteil der Sammlung zu verstehen, wäre mithin widersprüchlich, und ist gem. § 4 Abs. 2 S. 2 UrhG außerdem ausgeschlossen. Der Quellcode wird hier folglich aus der Begriffsdefinition des trainierten Netzes herausgenommen. Dennoch kann dieser freilich unabhängig davon einem Computerprogrammschutz gem. § 69a UrhG zugänglich sein. ${ }^{287}$

Funktionen, die lediglich zum Training des KNN erforderlich sind, gehören ebenfalls nicht zur Sammlung ,trainiertes Modell“. Nur dann, wenn das KNN zur Weiterentwicklung bereitgestellt werden sollte, könnte es sinnvoll sein,

286 Vgl. etwa Ehinger/Stiemerling, CR 12 2018, 768 f..

287 Vgl. dazu aa). 
auch diese Hyperparameter in die Sammlung aufzunehmen. Gleiches gilt dann entsprechend auch für den Quellcode.

\section{b) Unabhängige Elemente}

Fraglich erscheint, ob den einzelnen Elementen der Sammlung ein von den anderen Elementen unabhängiger Informationsgehalt zukommen muss. Dass sie selbst nicht auch Werke sein müssen, geht aus $§ 4$ Abs. 1 S. 1 UrhG hervor, der explizit von „Werken“, „Daten“ und ,,anderen unabhängigen Elementen“ spricht.

Nach dem Wortlaut des $\S 4$ Abs. 1 S. 1 UrhG müssen sämtliche Elemente jedenfalls ,unabhängig“ sein, wobei zu klären ist, welche Qualität diese Unabhängigkeit aufweisen muss.

Grundsätzlich sind die Elemente einer Sammlung unabhängig, wenn sie sich trennen lassen, ohne dass der Wert ihres informativen, literarischen, künstlerischen, musikalischen oder sonstigen Inhalts dadurch beeinträchtigt wird. ${ }^{288}$ Dieses Kriterium soll unter anderem verhindern, dass etwa Musikstücke als Sammelwerk ihrer Töne, oder ein Buch als Sammelwerk der Buchstaben geschützt würde. ${ }^{289}$ In diesen Fällen gewinnen die einzelnen Bestandteile ihren Sinn erst aus dem Kontext mit den anderen Elementen: Ein einzelner Ton etwa ergibt für den Hörer noch keinen „Sinn“, im Gegensatz zum Abspielen einer Phrase oder des gesamten Stücks (ggf. wäre zu untersuchen, ob sich diese Argumentation auch hält, wenn ein umfassendes Musikwerk - etwa eine Sinfonie - mit ihren einzelnen Passagen betrachtet wird, sodass die Bestandteile nicht einzelne Töne, sondern etwa musikalische Motive wären - auch Kombinationen können zumindest nach europäischer Rechtsprechung Elemente darstellen ${ }^{290}$ ). Die Elemente dürfen also nicht erst aus der Gesamtschau, sondern müssen auch alleinstehend Sinn ergeben, ${ }^{291}$ jedoch kann der Sinn auch durch ein ,formales Anordnungsprinzip erzeugt ${ }^{\text {‘292 }}$

288 EuGH GRUR 2005, 254, 255, Rn. 29 - Fixtures-Fußballspielpläne II; Schricker/Loewenheim-Leistner, Urheberrecht, § 4 Rn. 18.

289 Nordemann/Fromm-Czychowski, UrhR, § 4 Rn. 24; Dreier/Schulze-Dreier, UrhG, $\S 4$ Rn. 10.

290 EuGH GRUR 2005, 254, 255, Rn. 35 - Fixtures-Fußballspielpläne II; EuGH MMR 2016, 51, 52, Rn. 20 f. - Verlag Esterbauer.

291 Dreier/Schulze-Dreier, UrhG, § 4 Rn. 10; Schricker/Loewenheim-Leistner, Urheberrecht, § 4 Rn. 18.

292 Nordemann/Fromm-Czychowski, UrhR, § 4 Rn. 28. 
werden. Als Beispiel wird etwa eine Sammlung von Postleitzahlen herangezogen: ${ }^{293}$ eine einzelne fünfstellige Zahlenfolge, wie etwa 70839, ergibt für sich genommen wenig Sinn. Erst das Wissen darüber, dass es sich um eine Postleitzahl handelt, eröffnet Informationen darüber, dass etwa bestimmte Straßen diesem Postleitzahlenbereich zugeordnet sein könnten.

Bezüglich des Informationsgehaltes eines Elements ist darüber hinaus nicht maßgeblich, welcher Informationsgehalt dem Element nach der Zweckbestimmung der Sammlung zukäme, sondern jeder denkbare Informationswert. $^{294}$

Das Abrufen einzelner, wie oben definierter Elemente (abgestellt wird nicht nur auf die trainierten Parameter, sondern auch auf die Hyperparameter, vgl. ee)) aus einem KNN liefert Informationen über die verwendeten Aktivierungsfunktionen, die Anzahl der Schichten und alle weiteren Informationen, die in der Sammlung ,trainiertes Modell“ enthalten sein können. Jedes einzelne Element trifft dabei eine Aussage über das betreffende Modell. Diese Elemente haben also jeweils einen eigenen Aussage- bzw. Informationsgehalt und sind mithin unabhängig. ${ }^{295}$

Fraglich könnte sein, ob die Elemente einer Datenbank gleichartig sein müssen: Die Elemente der Sammlung ,trainiertes Modell“ können nicht nur Zahlenwerte sein (,Anzahl Schichten“, „Anzahl Neuronen“ etc.) sondern auch Funktionsnamen (Art der Aktivierungsfunktion, Kostenfunktion etc.) und auch Zusammenstellungen vieler Zahlenwerte in einem Element (Parameter). Denkbar wäre zu argumentieren, es handele sich bei allen Informationen zu einem einzigen trainierten KNN um lediglich einen Datensatz, und nur bei einer Zusammenstellung mehrerer trainierter Modelle könne von einer Sammlung gesprochen werden. Dem ist zu entgegnen, dass zwar in einer Briefmarkensammlung bestimmte Briefmarken gesammelt, und in einer Zeitschriftendatenbank bestimmte Zeitschriften erfasst sind, dass aber Sammlungen sich auch mit einem bestimmten Thema befassen können, das alle Elemente verbindet, so unterschiedlich diese im Einzelnen auch sein mögen, wie es etwa in Ausstellungen ${ }^{296}$ üblicherweise der Fall ist. Ferner kann auch schon ein einzelnes topografisches Datenblatt eine Datenbank

293 Nordemann/Fromm-Czychowski, UrhR, § 4 Rn. 26.

294 Dreier/Schulze-Dreier, UrhG, § 4 Rn. 18.

295 Die Unabhängigkeit „,der Elemente“ ablehnend Apel/Kaulartz, RDi Nr.1 2020, 24, 29, ohne jedoch die Elemente tiefergehend zu analysieren.

296 Vgl. z. B. LG München I ZUM-RD 2003, 492, 498 f. - Jemen-Ausstellung. 
darstellen. ${ }^{297}$ Die Verschiedenheit der Art der Elemente steht demzufolge hier einem Schutz nicht entgegen.

Die Sammlung ,trainiertes Modell“ besteht also aus unabhängigen Elementen im Sinne von $\S 4$ Abs. 1 S. 1 UrhG. ${ }^{298}$

\section{c) Systematische oder methodische Anordnung}

Gem. § 4 Abs. 2 S. 1 UrhG müssten die Elemente nicht nur unabhängig, sondern auch systematisch oder methodisch angeordnet sein. Erforderlich ist also, dass der Anordnung ein gewisses System zugrunde liegt, das auch in einem Ordnungsschema oder einer Klassifizierung bestehen kann, oder die Anordnung müsste - methodisch - auf einer ordnenden Handlungsanweisung oder einem Plan basieren. ${ }^{299}$

Wenn die Elemente in einer für das erneute Einlesen vorgesehenen Datei gesammelt vorliegen, ist bereits evident, dass eine Struktur vorhanden sein muss: Sonst wäre ein Abrufen der Daten nicht möglich. Die Strukturierung innerhalb der Dateien ergibt sich entweder aus Indizes oder aus Schlüsselwörtern, mit denen die einzelnen Elemente adressiert werden können. Wenn mehrere Dateien vorliegen, kann sich die Anordnung auch aus der Ordnerbzw. Verzeichnisstruktur ergeben.

297 LG München I GRUR 2006, 225, 227 - Topografische Kartenblätter; Wandtke/Bullinger-Hermes, PK UrhR, § 87a Rn. 32.

298 Randbemerkung: auch wenn eine einzelne Gewichtungsinformation (die stets in einer Kombination aus die dazugehörigen Neuronen identifizierenden Indizes ausgegeben wird) abgerufen würde, informiert diese immerhin darüber, wie an einer bestimmten Stelle des KNN die Verbindung zwischen zwei Neuronen gewichtet ist (ähnlich einer Postleitzahl in einer Postleitzahlenliste) - ein einzelner Parameter etwa als Zahl 0,42 - ergibt also zwar alleinstehend keinen tieferen Sinn. Wenn die Wahrnehmende allerdings weiß, dass es sich um einen Parameter eines KNN handelt, ist zumindest klar, dass in dem KNN an einer Stelle der Output eines Neurons mit dem Gewicht 0,42 als Input des darauffolgenden Neurons weitergeleitet wird, und könnte möglicherweise ebenso als unabhängiges Element angesehen werden. Dies kann vorliegend jedoch zunächst dahinstehen.

299 Dreier/Schulze-Dreier, UrhG, § 4 Rn. 17, Wandtke/Bullinger-Marquardt, PK UrhR, $\S 4$ Rn. 10. 


\section{d) Zugänglichkeit der Elemente}

$\S 4$ Abs. 2 S. 1 UrhG fordert ferner, dass die Elemente einzeln mit Hilfe elektronischer Mittel oder auf andere Weise zugänglich sind. Die Zugänglichkeit ist gegeben, wenn es möglich ist, auf die Elemente unter Berücksichtigung der Anordnungskriterien zuzugreifen und sie abfragen zu können. ${ }^{300}$

Jede Framework-Variante stellt Methoden bzw. Funktionen bereit, mithilfe derer die Elemente einzeln abgerufen werden können. Teils ist dieses frameworkübergreifend möglich, teilweise kann ein KNN nur genau so wieder geladen werden, wie es auch gespeichert wurde. Für alle gilt jedoch, dass der Vorgang des Abspeicherns darauf angelegt ist, die Daten wieder zu laden und zugreifbar zu machen. Erwähnung finden sollte an dieser Stelle noch, dass die KNNs ganz üblicherweise nicht als gepackte ausführbare Dateien (wie z. B. eine „Word.exe“, die nach einen Doppelklick das Programm startet) das Endergebnis des Trainingsvorgangs darstellen, sondern sie müssen - durch einen Aufruf in Code oder Skript - geladen werden und sind dann auch zugänglich und veränderbar.

\section{e) Zwischenergebnis}

An anderer Stelle wird die Datenbankqualität eines neuronalen Netzes kategorisch abgelehnt mit dem Argument, die „Daten der Verarbeitungsschicht“ - die Gewichte der Neuronen - interessierten den Endnutzer nicht, ferner sei ,ihr Wert als solcher für ihn auch keine verwertbare Information““.301 Hierbei wird jedoch übersehen, dass der ,Endnutzer“, zum Beispiel also der Verwender des trainierten DeepDream-Modells, ${ }^{302}$ nicht zwingend die hier zu betrachtende Zielgruppe darstellt.

Richtig ist, dass etwa die Verwenderin eines KNN zur Bildbearbeitung nicht an den einzelnen Neuronenwerten interessiert ist. Anders jedoch ist die Lage für den Entwickler, der ein vortrainiertes Netz - oder einzelne Schichten daraus - weiterverwenden möchte. Zudem wird verkannt, dass nicht nur die Neuronengewichte als Datenbankinhalt relevant sind, sondern die gesamte Netzwerkstruktur. Möglicherweise hat sich in den vergangenen 20 Jahren $^{303}$ jedoch auch der Stand der Technik derart verändert, dass die

300 Nordemann/Fromm-Czychowski, UrhR, § 4 Rn. 36.

301 Grützmacher, Datenbanken, S. 66; Ehinger/Stiemerling, CR 12 2018, 761, 769.

302 Vgl. § 10 C.I..

303 Die Aussagen von Grützmacher sind von 1999. 
Sichtweise nun eine andere zu sein hat. Insbesondere werden heute wohl häufig vortrainierte Modelle verwendet, es sind zahlreiche für bestimmte Analysezwecke (wie etwa die Gesichtserkennung) spezialisierte Modelle verfügbar, die den individuellen Anforderungen angepasst werden können. Insofern ist auch nicht (mehr) nur auf den Endnutzer im Sinne eines nicht an der Entwicklung beteiligten Benutzers abzustellen, sondern vielmehr auf Entwickler, die sich das Modell heraussuchen, das für ihre Zwecke besonders gut geeignet ist. Und für diese kann es mitunter sehr interessant sein, die „Daten der Verarbeitungsschicht" in Augenschein zu nehmen. Zudem ermöglicht eine rückwirkende Analyse dieser Daten einen Einblick in die Arbeitsweise des verwendeten Modells, etwa zur Fehlersuche oder zur Vermeidung von unzutreffenden Vorhersagen, die auf schlecht gewählten Trainingsdaten basieren: Im Rahmen dieser „KI-Erkläransätze“ (Stichwort: „Explainable AI“) können auch einzelne bzw. Gruppen von Neuronenwerten interessieren. ${ }^{304}$

Nicht zuletzt ist auch der Telos des Datenbankwerkschutzes zu berücksichtigen: Es ist nicht das Ziel, anhand der Qualität der Elemente einer Sammlung einen neuen Schutz der enthaltenen Elemente zu konstruieren. Das Gegenteil ist der Fall: Der Fokus liegt auf dem, was durch Auswahl und Anordnung geschaffen wird, und was gerade mehr als die Summe seiner Teile sein kann. Eine denkbare Parallele zu dem ungewünschten Zerlegen eines Buches oder Musikwerkes in seine Einzelteile ergibt sich hier nicht, denn die untersuchten ML-Modelle sind gerade nicht als Ganzes anderweitig urheberrechtlichem Schutz unterstellt. Vielmehr kommt ihnen aus den dargelegten Ausführungen viel eher der Charakter einer Datenbank zu, die Informationen enthält, die schlussendlich von Computerprogrammcode eingelesen und für die Erzeugung neuer Bilder oder Tonfolgen oder der Zuordnung von Kategorien eingesetzt werden.

Wie gezeigt, kann - bei entsprechender Definition des Begriffs des trainierten KNN - grundsätzlich eine Datenbank vorliegen. Die Werkqualität im Sinne einer persönlichen geistigen Schöpfung ist jedoch gesondert zu prüfen.

304 Vgl. z. B. die sog. „Sentiment-Analysis“ zur Erkennung negativer Formulierungen in Texten, die die Gewichte einer „Attention“-Schicht besonders hervorhebt, https: //traversals.com/blog/explainable-ai-for-sentiment-analysis/ (Stand: 22.02.2021) Gewichte mit dem Wert 0 können u. a. darauf hinweisen, dass zu untersuchende Eigenschaften gar nicht vorlagen bzw. Pfade im Netz blockiert sind, Ancona et al., Gradient-Based Attribution Methods, S. 179. 


\section{Persönliche geistige Schöpfung}

Spätestens bei der Anforderung der persönlichen geistigen Schöpfung scheitern bisherige Subsumtionsversuche, ${ }^{305}$ die ihre Prüfung jedoch auf eine Sammlung von Parametern (Gewichtungsinformationen) beschränken. Möglicherweise ergibt sich ein anderes Ergebnis, wenn das wie hier beschriebene gesamte trainierte KNN als Untersuchungsgegenstand herangezogen wird.

Die Anforderung der Werkqualität für Datenbankwerke ergibt sich - wenn nicht schon aus dem Begriff - zumindest daraus, dass $\$ 4$ Abs. 2 UrhG Datenbankwerke als Sammelwerke mit erweiterten Eigenschaften definiert. Die Sammelwerke wiederum finden ihre Legaldefinition in $\S 4$ Abs. 1 UrhG, und dort heißt es: „Sammelwerke sind Sammlungen von Werken, (...) die aufgrund der Auswahl oder Anordnung der Elemente eine persönliche geistige Schöpfung sind.“

\section{a) Persönliche oder eigene geistige Schöpfung?}

In Art. 3 Abs. 1 Datenbank-RL ist hingegen von einer ,eigenen geistigen Schöpfung" die Rede, sodass zu fragen ist, ob hierin ein Unterschied zur „persönlichen geistigen Schöpfung“ besteht und worin dieser liegt. Grützmacher ${ }^{306}$ war noch der Ansicht, dass hierin ein maßgeblicher Unterschied bestünde, inzwischen ist jedoch weitgehend geklärt, dass der deutsche Gesetzgeber es schlicht nicht für erforderlich hielt, den Wortlaut in § 4 UrhG anzupassen (anders als in $\S 69 \mathrm{a}$ Abs. 3 UrhG, in den die ,eigene“ geistige Schöpfung Eingang gefunden hat), mit der Begründung, dass bereits vor Erlass der Richtlinie keine erhöhten Anforderungen (erhöht im Sinne einer ,persönlichen“ gegenüber einer „eigenen“ geistigen Schöpfung) gestellt wurden. ${ }^{307}$ Inzwischen hat auch der EuGH in der Football Dataco/YahooEntscheidung $^{308}$ die Anforderungen an den Datenbankschutz explizit in die-

305 Ehinger/Stiemerling, CR 12 2018, Rn. 62 lassen es letztendlich an der persönlichen geistigen Schöpfung scheitern, Hartmann/Prinz, WRP 12 2018, Rn. 62 steigen schon bei der Anforderung an die Unabhängigkeit der Elemente aus und prüfen die persönliche geistige Schöpfung nicht mehr.

306 Grützmacher, Datenbanken, S. $181 \mathrm{ff.}$.

307 Ahlberg/Götting-Ahlberg, BeckOK-UrhG, § 4 Rn. 25; Dreier/Schulze-Dreier, UrhG, § 4 Rn. 11; BT-Drs. 13/7385.

308 EuGH GRUR 2012, 386, 387- Football Dataco/Yahoo. 
sem Sinne geklärt. Fraglich ist also nur, welche Anforderungen diesbezüglich an die hier untersuchten elektronischen Datenbankwerke zu stellen sind.

b) Schöpfung in Auswahl oder Anordnung

Gem. §4 Abs. 2 UrhG i. V. m. § 4 Abs. 1 UrhG müssten die Anordnung oder Auswahl der Elemente die persönliche geistige Schöpfung begründen.

aa) Auswahl

Auswahl ist das Sichten, Sammeln, Bewerten und Zusammenstellen unter Berücksichtigung besonderer Auslesekriterien, ${ }^{309}$ wobei ein Entscheidungsspielraum hinsichtlich der Auswahlmöglichkeit erforderlich ist, damit ein Datenbankwerk im Sinne einer geistigen Schöpfung entstehen kann. ${ }^{310}$

\section{bb) Anordnung}

Anordnung hingegen meint die Einteilung, Präsentation und Zugänglichmachung der ausgewählten Elemente nach einem oder mehreren Ordnungssystemen. ${ }^{311}$

cc) Anordnung für Datenbankwerke i. d. R. programmseitig vorgegeben

Naturgemäß ergibt sich die konkrete Anordnung der Elemente von Datenbankwerken aus den technischen Gegebenheiten und ist bei „klassischen“ elektronischen Datenbanken überwiegend von der Datenbanksoftware vorgegeben. ${ }^{312}$ Es wird daher auf das Ausgabeformat der Daten abgestellt, also darauf, wie die Daten in der Ausgabe angeordnet sind. Die Elemente müssten systematisch und methodisch zugänglich sein, ${ }^{313}$ sodass es für die Anord-

309 Dreier/Schulze-Dreier, UrhG, § 4 Rn. 11.

310 Wandtke/Bullinger-Marquardt, PK UrhR, § 4 Rn. 9.

311 Dreier/Schulze-Dreier, UrhG, § 4 Rn. 11.

312 Dreier/Schulze-Dreier, UrhG, § 4 Rn. 19.

313 Dreier/Schulze-Dreier, UrhG, § 4 Rn. 19. 
nung auf die Schöpfungshöhe der Ausgabe- und Verknüpfungsmöglichkeiten ankommt. ${ }^{314}$

Spätestens an dieser Stelle könnte fraglich erscheinen, wie das oben mühevoll konstruierte ML-Datenbank,,werk“ überhaupt mit einer elektronischen Datenbank, wie sie allgemein in der Vorstellung existiert, zu vergleichen sein könnte, entspricht eine Speicherung von Objekten im Binärformat doch nicht der klassischen Interpretation des Begriffes „Datenbank“, bei dem Daten in einer Eingabemaske gesucht oder in Form eines Indexes dargestellt werden können.

Das Verständnis erleichtert ein Blick hinter die Kulisse einer solchen „klassischen“ Datenbank: Auch diese legt Informationen gewöhnlicherweise in einer oder mehreren Dateien ab, die dann - bei Aufruf - eingelesen und etwa einem im Code definierten Tabellenobjekt zugewiesen werden (wenn nicht schon Tabellenobjekte in serialisierter Form abgelegt wurden - dann ist die Parallele noch offensichtlicher). Dieses Tabellenobjekt enthält dann Informationen unter anderem darüber, wie es heißt, wie viele Spalten es enthält, wie diese Spalten heißen, und was ihr Inhalt ist. Wie diese Datenbank vom Menschen wahrgenommen werden kann, hängt davon ab, wie sie zum Beispiel im Browser präsentiert wird.

Folglich könnte auch ein trainiertes KNN, das als Objekt oder anderweitig in Dateien abgelegt wurde, in einer Form wiedergegeben werden, die es vermutlich einfacher machen würde, darin eine Datenbank zu erkennen. Auch bei trainierten KNN hat der Entwickler aber bis auf die Auswahl der verwendeten Frameworks keinen oder wenig Einfluss darauf, wie die Elemente angeordnet werden, sodass es für die persönliche geistige Schöpfung auf die Auswahl der Elemente ankommen muss.

dd) Schöpfungsspielraum in der Auswahl

Hinsichtlich dieser Auswahl ist die Konzeption derselben entscheidend, nicht jedoch, dass der Urheber die zur Durchführung der Auswahl erforderlichen Schritte selbst vornimmt. ${ }^{315}$

314 Dreier/Schulze-Dreier, UrhG, § 4 Rn. 19, Schricker/Loewenheim-Leistner, UrhR, $\S 4$ Rn. 34.

315 BGH GRUR 2007, 685 Rn. 19, 23 - Gedichttitelliste I; Dreier/Schulze-Dreier, UrhG, § 4 Rn. 19. 
Der Ersteller eines trainierten KNN ist in der Regel nicht Urheber der Abfragemöglichkeiten (diese Funktionalität wird durch die Frameworks bereitgestellt und vorgegeben). Durch die Auswahl der Speichervariante und des Frameworks wird jedoch zumindest insoweit Einfluss auf die „Abfrageund Verknüpfungsmöglichkeiten“ genommen, als es bei den zur Wahl stehenden Frameworks Unterschiede in dieser Hinsicht gibt. Zudem könnte differenziert werden, ob der Entwickler die Default-Speichervariante wählt dann würde die Auswahl der zu speichernden Elemente durch das Framework getroffen, was gegen eine persönliche geistige Schöpfung spräche - oder ob etwa Variablen gezielt benannt und zur Speicherung ausgewählt werden.

Allerdings kann für diese Prüfung durchaus auch auf einen noch früheren Zeitpunkt abgestellt werden: Der Entwickler wählt die Architektur des Netzes, die Funktionen, die Anzahl Trainingsdurchläufe etc. selbst aus, sodass sich das trainierte KNN als Ganzes als das Werk präsentiert, dessen Elemente einzeln abrufbar sind. Dem Argument, die Auswahlmöglichkeiten seien doch auf die infrage kommenden Werte für die Hyperparameter beschränkt, ist entgegenzuhalten, dass auch in der Musik der Tonraum im Wesentlichen auf 8 Töne (in verschiedenen Oktaven) beschränkt ist. Dennoch ergeben sich durch das Hinzuziehen von Rhythmus und Dynamik einzigartige Kombinationen, die als geistige Schöpfungen anerkannt sind. Insbesondere die Wahl der Hyperparameter hat wesentlichen Einfluss auf die Leistungsfähigkeit des Netzes und erfordert geistige Anstrengung und Kreativität, denn der Entwickler des KNN hat in der Regel eine konkrete Vorstellung davon, was das KNN leisten können soll, und nimmt aufwendige Anpassungen vor, die sich letztendlich in der Kombination aus Hyperparametern und Parametern niederschlagen. Mithin ist auch ein tagelanges Training eines KNN nutzlos, wenn die Hyperparameter nicht sorgfältigst gewählt wurden.

Solange der Entwickler sich also nicht Automatismen bedient, um Hyperparameter zu optimieren, sondern diese selbst wählt, dem KNN-Objekt zuweist, dieses ,trainiert“ und das gesamte Ergebnis der Reproduzierbarkeit halber speichert, spielt in einem trainierten KNN die Auswahl der Datenbankinhalte durch den Entwickler selbst eine derart bedeutende Rolle, dass beim Ergebnis i.d.R. von einer persönlichen geistigen Schöpfung ausgegangen werden kann. Selbstverständlich kann hier die Prüfung in Einzelfällen zu anderen Ergebnissen kommen, wenn etwa ein vortrainiertes Netz verwendet und angepasst wird, oder wenn der Beitrag des Urhebers nicht über die Reproduktion bekannter banaler Strukturen hinausgeht. 


\section{Ergebnis}

Ein wie hier definiertes trainiertes KNN - ohne den aufrufenden Quellcode erfüllt alle Anforderungen an ein Datenbankwerk und kann in der Auswahl der Elemente auch eine persönliche geistige Schöpfung darstellen, sodass es dem Schutz für Datenbankwerke gem. § 4 Abs. 2 UrhG grundsätzlich zugänglich ist.

\section{Wer ist der Urheber?, oder: Schutzumfang und Folgen}

Sobald die grundsätzliche Schutzfähigkeit festgestellt wurde, stellt sich unweigerlich die nächste Frage: wem gebührt der Schutz? Das Vorliegen einer persönlichen geistigen Schöpfung wurde bejaht aufgrund der Auswahl der Hyperparameter. Urheber ist folglich, wer die Netzwerkstruktur, Aktivierungsfunktion etc. festlegt (nicht, wer eine vorgegebene Architektur lediglich umsetzt). Zu überlegen wäre noch, inwiefern eine ggf. davon verschiedene Person, die die Trainingsdaten auswählt, unter Umständen als Miturheber anzusehen ist. Bei einer Personenmehrheit muss an der Stelle allerdings berücksichtigt werden, dass die Auswahl (und ggf. auch die Aufbereitung bzw. Vorbereitung) der Trainingsdaten höchstens einen Rahmen setzen kann. Die geistige Schöpfung liegt immer noch darin, die an die Trainingsdaten angepasste und die zur Erreichung der Aufgabenstellung optimale Kombination an Netzwerkeinstellungen auszuwählen.

Der Schutzumfang ist sorgfältig zu begrenzen, um ihn nicht in einen Ideenschutz ausufern zu lassen. Sinnvoll erscheint eine Begrenzung auf die konkrete Gestalt des trainierten KNN. Welche Gestalt dieses annehmen kann, wurde zu Beginn des Kapitels bereits dargestellt. Die beschriebenen Formen - in der Regel Computerdateien, eine einzelne oder mehrere - sind der Gegenstand, den es vor Vervielfältigung zu schützen gilt.

\section{Investitionsschutz gem. $\S \S 87 \mathrm{a}$ ff. UrhG}

Mit den Regelungen in $\S \S 87$ a ff. UrhG wird in Umsetzung der DatenbankRL demjenigen, der eine Datenbank unter Aufwendung einer nach Art oder Umfang wesentlichen Investition erschafft, ein 15-jähriger sui-generis-Schutz gewährt. Dieser kann grundsätzlich auch neben einem urheberrechtlichen 
Schutz aus $\S 4$ Abs. 2 UrhG i. V. m. $§ 4$ Abs. 1 UrhG bestehen. ${ }^{316}$ Folglich sollen seine Voraussetzungen auch hier für trainierte KNN geprüft werden. Auch hier wird der aufrufende Quellcode wieder außer Acht gelassen. Zu prüfen ist, ob eine Datenbank sowie ein Investitionsgegenstand im Sinne des $\S 87$ a UrhG vorliegen und ob die Wesentlichkeit der Investition gegeben ist.

\section{Datenbank}

Während die Datenbank-RL einen einheitlichen Datenbankbegriff für den urheberrechtlichen ebenso wie den sui-generis-Schutz verwendet, umschreibt der deutsche Gesetzgeber Datenbanken in § 4 UrhG und § 87a UrhG jeweils unterschiedlich, die Voraussetzungen stimmen letztendlich jedoch überein. ${ }^{317}$ Wenn eine Datenbank nach $\S 4$ UrhG gegeben ist, kann also auch für $\S 87$ a UrhG vom Vorliegen einer Datenbank ausgegangen werden.

Im Rahmen der urheberrechtlichen Prüfung in e) wurde bereits festgestellt, dass ein trainiertes künstliches neuronales Netz Datenbankqualität aufweist, insofern erübrigt sich die Prüfung an dieser Stelle.

\section{Investitionsgegenstand}

Zusätzlich muss für § 87a UrhG im Rahmen der Beschaffung, Sammlung, Überprüfung oder Darstellung der Datenbankinhalte eine nach Art oder Umfang wesentliche Investition anfallen (§ 87a Abs. $1 \mathrm{~S} .1 \mathrm{UrhG})$. Sowohl finanzielle Mittel als auch der Einsatz von Zeit, Arbeit und Energie können die Investitionen ausmachen. ${ }^{318}$ Nicht berücksichtigt werden Kosten für die Erzeugung von Daten. ${ }^{319}$

Für die Identifizierung der Investition in die KNN-Entwicklung bzw. in die Erstellung eines KNN-Modells - also der Datenbank - ist es hilfreich, den Entstehungsprozess erneut unter die Lupe zu nehmen.

Der Entwickler wählt anhand der Aufgabenstellung und seiner Erfahrung initiale Hyperparameter aus, mithilfe derer das Modell trainiert wird. Am Ende des Trainingsvorgangs wird evaluiert, ob die Ergebnisse den Vorstellungen genügen. In diesem Zeitpunkt entsteht bereits eine erste Vorstufe

316 Dreier/Schulze-Dreier, UrhG, Vorbemerkung zu § 87a, Rn. 8.

317 Dreier/Schulze-Dreier, UrhG, § 87a Rn. 3.

318 ErwGr. 40 Datenbank-RL

319 Vgl. Dreier/Schulze-Dreier, UrhG, § 87a Rn. 13. 
der Datenbank. Anschließend passt der Entwickler die Hyperparameter an und wiederholt den Trainingsvorgang, und wiederholt diesen Prozess, bis die Ergebnisse passen (z. B. bis die Genauigkeit einer bestimmten Mindestprozentzahl entspricht, bzw. bis der Entwickler den Eindruck hat, dass das Modell seine geistige Vorstellung der Problemlösung hinreichend abbildet). Insofern könnte dieser Vorgang als Datenbeschaffung oder Datensammlung (Daten sind hier nicht die Trainingsdaten, sondern eben die Elemente der entstehenden Datenbank) bezeichnet werden. Die eigentliche, persistente, nutzbare Datenbank entsteht erst, wenn der Entwickler sich dazu entschließt, die gesammelten Daten nicht zu verwerfen, indem zumindest Checkpoints ${ }^{320}$ erstellt werden bzw. am Ende des Trainingsprozesses das Modell reproduzierbar im Speicher ablegt wird.

Es wird hier insbesondere nicht auf die Berechnung und Optimierung der Parameter abgestellt im Sinne einer Erzeugung neuer Daten. Die dafür entstehenden Kosten wären kein tauglicher Investitionsgegenstand. Vielmehr wird davon ausgegangen, dass die Zusammenhänge, die der Entwickler in den Daten vermutet, bereits bestehen und lediglich greifbar gemacht werden müssen, um daraus zum Beispiel Aussagen für die Zukunft treffen zu können. Die Muster in den Daten, mit denen die ML-Modelle arbeiten, werden nicht erst hergestellt, sondern aufgefunden.

\section{Wesentlichkeit der Investition}

Sodann ist zu klären, welche Kosten im Zusammenhang mit der Datenbankherstellung im Machine Learning-Kontext entstehen. Wird davon ausgegangen, dass lediglich Zusammenhänge zwischen bestehenden Daten ermittelt werden, so wären zumindest die Kosten für das „Ermittlungsprogramm“also den Algorithmus, bzw. die Entwicklung des Modells, sowie die erforderliche (Spezial-)Hardware (oder alternativ gemietete Online-Ressourcen) und das Gehalt für die das Modell trainierenden Data Scientists - zu berücksichtigen. Ferner dürften auch die Kosten für die Bereitstellung des Modells in Ansatz zu bringen sein. Auch die Beschaffung der Trainingsdaten - so

320 Checkpoints bezeichnen Speicherungen des Modells bzw. seiner Parameter, die die Wiederaufnahme des Trainings zu einem späteren Zeitpunkt ermöglichen, vgl. z. B. https://www.tensorflow.org/guide/checkpoint (Stand: 22.02.2021). 
sie entgeltlich erfolgt - und damit auch der Aufwand für ihre Sammlung, dürften relevante Kosten darstellen. ${ }^{321}$

Anforderungen an das Merkmal der Wesentlichkeit, bzw. ab welchem Betrag, welchem Zeitaufwand oder welcher Qualität an Investition Wesentlichkeit gegeben ist, gehen weder aus § 87a UrhG noch aus der Datenbank-RL hervor. ${ }^{322}$ Die Auslegung dieses unbestimmten Rechtsbegriffs ist vielmehr als „flexibles Kriterium“323 der Rechtsprechung überlassen. Es liegt nahe, die Auslegung an dem Ziel der Datenbank-RL zu orientieren, und einen Schutz zu schaffen, der einen Anreiz für die Entwicklung solcher Speicher- und Verarbeitungssysteme bietet, und damit die Schwelle zur Wesentlichkeit nicht zu hoch anzusetzen, ${ }^{324}$ sodass lediglich sog. ,Allerweltsinvestitionen“325 nicht erfasst sein sollen. Insbesondere sind keine Investitionen von substantiellem Gewicht vorausgesetzt. ${ }^{326}$ Es sind also Einzelfallentscheidungen zur Auslegung des Wesentlichkeitskriteriums erforderlich. ${ }^{327}$

Es ist jedoch davon auszugehen, dass in Anbetracht des nicht unerheblichen finanziellen und zeitlichen Entwicklungsaufwands zumindest für komplexe, tiefere neuronale Netze die Wesentlichkeit der Investition gegeben sein dürfte. Im Zweifel muss es hier auf eine Einzelfallbetrachtung ankommen.

321 So wird etwa über die einzigartige Zusammenstellung von Informationen zu global erteilten Patenten von IFSCLAIMS berichtet, dass beispielsweise die Vereinheitlichung der Firmennamen besonders aufwendig sei, die im Rahmen der Standardisierung der Trainingsdaten vorzunehmen ist - so sei durch jahrelange Arbeit ein einzigartiges Datenset entstanden, vgl. https://www.cmswire.com/informationmanagement/machine-learning-datasets-build-or-buy/ (Stand: 22.02.2021); auch der Zugang zu wertvollen Datensammlungen wie etwa die Inhalte von Beck-Online oder Juris erfordert in der Regel kostenpflichtige Abonnements.

322 Dreier/Schulze-Dreier, UrhG, § 87a Rn. 11; Wandtke/Bullinger-Hermes, PK UrhR, $\S 87$ a Rn. 52.

323 Wandtke/Bullinger-Hermes, PK UrhR, § 87a Rn. 52.

324 BGH GRUR 2011, 724, 725 - Zweite Zahnarztmeinung II; OLG Hamburg CR 2018, 22.

325 Wandtke/Bullinger-Hermes, PK UrhR, § 87a Rn. 54.

326 BGH GRUR 2011, 724, 725 - Zweite Zahnarztmeinung II Rn. 23; Dreier/SchulzeDreier, UrhG, § 87a Rn. 14; Haberstumpf, GRUR 2003, 20, 26.

327 BT-Drs. 13/7385 S. 45. 


\section{Ergebnis}

Der Hersteller eines trainierten KNN kann also als Datenbankhersteller auch in den Genuss des sui-generis-Datenbankherstellerschutzes gem. § 87a UrhG kommen. ${ }^{328}$ Dieser erstreckt sich nicht auf den Quellcode.

\section{Schutzumfang und Folgen}

Der Schutzumfang des Datenbankherstellerrechtes ergibt sich aus $\S 87 \mathrm{~b}$ Abs. 1 UrhG. Danach hat der Datenbankhersteller das ausschließliche Recht, die Datenbank insgesamt oder einen nach Art oder Umfang wesentlichen Teil der Datenbank zu vervielfältigen, zu verbreiten und öffentlich wiederzugeben. Datenbankhersteller ist, wer ,die Initiative ergreift [die Datenbank herzustellen] und das Investitionsrisiko trägt", ${ }^{329}$ mithin ist der Schutzinhaber nicht notwendigerweise identisch mit dem Urheber des Datenbankwerkes gem. § 4 Abs. 2 UrhG - ein Beispiel wäre ein Arbeitgeber-ArbeitnehmerVerhältnis, in dem der Arbeitgeber eine Idee für ein ML-Modell und eine zu erfüllende Aufgabe entwickelt, aber seine Entwicklungsabteilung mit der Konzeption, der Datensammlung und dem Training des ML-Modells betraut.

\section{Schutz als Computerprogramm gem. § 69 a UrhG}

Möglicherweise kommt einem trainierten ML-Modell in Python auch der Schutz für Computerprogramme gem. § 69a UrhG zu. ${ }^{330}$ In der oben (§ 6 D.III.) gefundenen Definition für trainierte KNN ist der Quellcode als ein wesentlicher Bestandteil des trainierten Modells genannt, wurde jedoch für den Datenbank(werk)schutz nicht berücksichtigt. Für den Schutz nach $\S 69$ a UrhG hingegen ist der Code - wie sich zeigen wird - von hoher Relevanz.

328 So wohl auch Söbbing, MMR 2021, 111, 114.

329 Erw.-Gr. 41 Datenbank-RL; Dreier/Schulze-Dreier, UrhG, § 87a Rn. 19.

330 Diskutiert wird das unter anderem von Hartmann/Prinz, WRP 12 2018, 1431, 1436 Rn. 47 ff.. und Ehinger/Stiemerling, CR 12 2018, 761, 764, Rn. 34 ff., insb. S. 767 Rn. 51, sowie Grätz, Künstliche Intelligenz im Urheberrecht, S. 46 ff.. 
1. Trainiertes Modell als Computerprogramm

Damit ein Schutz nach $\S 69$ a UrhG in Betracht kommt, muss ein Computerprogramm vorliegen. Weder das UrhG noch die durch $\S \S 69$ a ff. UrhG umgesetzte Computerprogramm-RL stellen eine Erklärung für die Bedeutung des Begriffes bereit, § 69a Abs. 1 UrhG spricht lediglich von „Programmen in jeder Gestalt“. Infolgedessen sind andere Quellen für eine mögliche Definition zu suchen.

\section{a) Begriffsklärung Computerprogramm}

Anhaltspunkte liefern die DIN 44300 sowie die Definition der WIPO und das IEEE Standard Glossary:

- Ein Computerprogramm ist ,eine Folge von Befehlen, die nach Aufnahme in einen maschinenlesbaren Träger fähig sind zu bewirken, dass eine Maschine mit informationsverarbeitenden Fähigkeiten eine bestimmte Funktion oder Aufgabe oder ein bestimmtes Ergebnis anzeigt, ausführt oder erzielt." ${ }^{\text {331 }}$

- Ein Computerprogramm ist ,eine zur Lösung einer Aufgabe vollständige Anweisung zusammen mit allen erforderlichen Vereinbarungen. “332

- Ein Computerprogramm ist „eine Kombination aus Computerinstruktionen und Datendefinitionen, die Computerhardware dazu befähigen, berechnende oder kontrollierende Funktionen auszuführen.“333

Mancherorts wird „Computerprogramm“ zudem abgegrenzt zu „Software“. Software soll dann alle digitalisierten Daten erfassen, also zwar auch Computerprogramme „im technischen Sinne“, aber darüber hinaus auch Texte, Grafiken, Musikdateien und andere Daten. ${ }^{334}$ Einigkeit scheint darüber zu be-

331 WIPO-Mustervorschriften, GRUR 1979, 306, § 1 (i); ebenso BGH GRUR 1985, 1041, 1047 - Inkasso-Programm.

332 Vgl. DIN 44300; Spindler/Schuster-Wiebe, Recht der elektronischen Medien, § 69a Rn. 3.

333 Institute of Electrical and Electronics Engineers, IEEE Standard Glossary of Software Engineering Terminology, S. 19.

334 Wandtke/Bullinger-Grützmacher, PK UrhR, § 69a Rn. 2; Software als auch Computerprogramme umfassender, aber weiterer Begriff Institute of Electrical and Electronics Engineers, IEEE Standard Glossary of Software Engineering Terminology, S. 66. 
stehen, dass es zur Abgrenzung für das Vorliegen eines Computerprogramms maßgeblich auf das „Vorhandensein von Befehls- und Steuerungsfunktionen“ ankommt, ${ }^{335}$ so verlangen auch die genannten Definitionen ,eine Folge von Befehlen“ bzw. eine „Anweisung“.

b) (Keine) Einordnung von ML-Modellen als Computerprogramm in der Literatur

Teilweise wird der Schutz insbesondere künstlicher neuronaler Netze als Computerprogramm in einer Parallele zur Wissensbasis von Expertensystemen $^{336}$ kategorisch abgelehnt, ohne eine weitere Prüfung vorzunehmen. ${ }^{337}$ An dieser Stelle soll jedoch eine differenziertere Betrachtung erfolgen.

Andernorts wird kritisiert, dass die „,bestimmte Funktion“, die die Definition der WIPO und damit auch der BGH verlangen, durch das ",trainierte Netz“ nicht gegeben sei, da sich die „Funktion“ des ,trainierten Netzes“ im Rahmen des Trainingsvorgangs verändere. ${ }^{338}$ Das scheint schon deshalb unpräzise, weil mit dem ,trainierten Netz“ der finale Zustand des KNN gemeint ist, und nicht der, der sich im Rahmen des Trainings noch verändert. ${ }^{339}$

Zudem seien die „Ausgabeparameter allein von den Eingangswerten abhängig“, ${ }^{340}$ weshalb das trainierte Netz dem „Datenaufbereiter“ zuzurechnen sei. $^{341}$

Möglicherweise wird hier zum einen verkannt, dass das „trainierte Netz“ (zumindest nach dem hiesigen Verständnis des trainierten Modells) eben nicht nur - einmalig und flüchtig - in Form des Maschinencodes oder Bytecodes vorliegt, sondern vielmehr auf die Elemente abzustellen ist, die es

335 Wandtke/Bullinger-Grützmacher, PK UrhR, § 69a Rn. 3; Dreier/Schulze-Dreier, UrhG, § 69a Rn. 12; DKM-Kotthoff, HK-UrhG, § 69a Rn. 5; OLG Rostock MMR 2008, 116.

336 Vgl. § 2 B.IV.2..

337 Wandtke/Bullinger-Grützmacher, PK UrhR, § 69a Rn. 21.

338 Hartmann/Prinz, WRP 12 2018, 1431, 1436; Linke, GRUR Junge Wissenschaft 2019, S. 42.

339 Ein Unterschied könnte sich allenfalls im sogenannten „Online-Learning“ ergeben: In dieser Variante wird ein Modell trainiert und implementiert, das dann aber im Praxiseinsatz weiter dazulernt (zur Begriffsklärung ,online“ in diesem Kontext vgl. Goodfellow et al., Deep Learning Handbuch, S. 310). Aber auch in diesem Fall müsste die ,bestimmte Funktion“ für den tauglichen Einsatz bereits bestehen.

340 Hartmann/Prinz, WRP 12 2018, 1431, 1437.

341 Dies., WRP 12 2018, 1431, 1437. 
ermöglichen, das trainierte Netz auch einzusetzen. Das Verhältnis zwischen den Komponenten des wie hier definierten trainierten KNN und dem im Zuge der Ausführung entstehenden Maschinencode ist nicht anders zu beurteilen als das Verhältnis zwischen regulärem Quellcode und Maschinencode anderer Programme.

Zum anderen wird nicht berücksichtigt, dass die Ausgabeparameter zwar zu einem großen Teil von den Trainingsdaten abhängen, dass aber die Hyperparameter bzw. die Modellstruktur auch einen maßgeblichen Teil zum Ergebnis beitragen. Die Leistung allein auf den „Datenaufbereiter“ zu begrenzen und dabei die Expertise unter anderem desjenigen, der die Hyperparameter wählt, außer Acht zu lassen, scheint zu kurz gegriffen.

\section{c) Berücksichtigung der Bestandteile eines trainierten Modells für den Computerprogrammbegriff}

Zu klären ist deshalb erneut, allerdings diesmal konkret in Bezug auf den Computerprogrammschutz, auf welche Komponente(n) des trainierten Modells bei einer Subsumtion unter $\S 69$ a UrhG abzustellen ist. Denkbare Anknüpfungspunkte wären der Quellcode, die erzeugten Dateien, sowie eine zur Laufzeit kombinierte Variante (im Ergebnis ähnlich Maschinencode) derselben. Für den Quellcode ist sauber zu trennen zwischen dem Quellcode, der für das Training des Modells verwendet wird, und dem Quellcode, der das trainierte Modell lädt und einsetzt. Für den Schutz des trainierten $K N N$ als Computerprogramm ist ausschließlich letzterer relevant, denn das andere Programm hat eine ganz andere Zielrichtung bzw. Zwecksetzung, und möglicherweise auch eine sehr verschiedene Komplexität: Während der Code zur Erzeugung eines Modells Anweisungen zur Aufbereitung der Trainingsdaten, evtl. auch zu Funktionalitäten der einzelnen Modellbestandteile sowie Testprozeduren enthält, genügen für den Quellcode, der das trainierte Modell einsetzt, mitunter wenige Zeilen Programmcode, in denen das Modell und die Eingabedaten geladen und eine Vorhersage ausgeführt und ausgegeben wird (teilweise ist sogar eine einzeilige Skripteingabe in einer Kommandozeile ausreichend ${ }^{342}$, allerdings hängt die Komplexität sowohl des Trainings- als auch des Ausführungscodes stark von der Aufgabenstellung und der individuellen Implementierung ab).

342 Vgl. https://www.tensorflow.org/guide/saved_model\#run_command (Stand: 22.02.2021). 


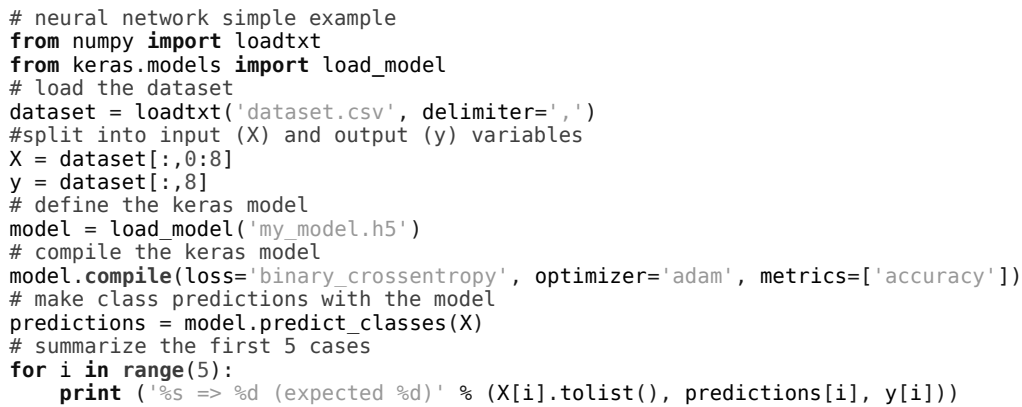

Abbildung 7.1: Einfaches Beispiel in Keras: Laden eines Modells aus .h5-Datei, Quelle (leicht abgewandelt): https://machinelearningmastery.com/tutorial-first-neural-network-pythonkeras/, 22.02.2021.

In Bezug auf den besagten Quellcode ergeben sich verschiedene Gestaltungsmöglichkeiten: So kann dieser die für den Produktiveinsatz erforderliche Struktur des Modells aufbauen, in dem der Programmierer die im Training ermittelten und optimierten Hyperparameter im Code fest vorgibt, und lediglich die Parameter aus Dateien nachladen, oder aber auch die Hyperparameter etwa im JSON-Format ${ }^{343}$ bereitgestellt bekommen. Auch eine dritte Variante ist denkbar, aber praxisfern, bzw. nur für Testzwecke praktikabel: Der Programmierer könnte im Code zum einen die Hyperparameter festlegen, zum anderen das Modell zufällig mit Parametern versehen, das Training durchführen und im Anschluss (ohne das Modell persistent zu speichern) das Modell direkt zum vorgesehenen Einsatzzweck verwenden. Dies hätte allerdings eine enorm begrenzte, wenn nicht gar unmögliche Reproduzierbarkeit dieses Ergebnisses zur Folge. Das Beispiel soll nur verdeutlichen, welche Rolle der Quellcode beim Einsatz eines trainierten Modells einnehmen kann. Der Unterschied der dargestellten Varianten schlägt sich naturgemäß vor allem in der Komplexität des Codes nieder.

Abbildung 7.1 zeigt ein Minimalbeispiel für Quellcode in Keras, bei dem das Modell zuvor in einer . h5-Datei gespeichert wurde. In Zeile 6 werden die Testdaten eingelesen, die zuvor im CSV-Format (Comma-Separated-Values-

343 JSON (Java Script Object Notation) ist ein verbreitetes Format zur Strukturierung von Daten zur Datenbereitstellung bzw. Datenaustausch, das von vielen Programmiersprachen verarbeitet werden kann, für weitere Informationen vgl. https://www.json.org/json-de.html (Stand: 22.02.2021). 
Format) zusammengetragen wurden. Diese werden für eine Vorhersage in Zeile 14 verwendet. $X$ repräsentiert eine Sammlung von Testdaten, y die dazugehörigen Labels. In Zeile 10 wird das gespeicherte Modell eingelesen. Resultat ist bzw. sind die vom Modell gefundenen Labels für die eingegebenen Testdaten X. ${ }^{344}$

Ziel des folgenden Abschnitts ist, herauszustellen, welche Kombination von Elementen eines trainierten Modells mindestens gegeben sein muss, damit die Anforderungen an ein „Computerprogramm“ im Sinne des § 69a UrhG erfüllt sind.

\section{aa) Quellcode}

Es liegt nahe, den Quellcode für die Subsumtion unter § 69a UrhG heranzuziehen. Quellcode meint dabei nur den für die konkrete Problemlösung entworfenen Code, die verwendeten (,importierten“) Frameworks mit ihren Klassen und Funktionen sind hier grundsätzlich außer Acht zu lassen, in Bezug auf diese kann nur die Auswahl der eingesetzten Klassen und Funktionen relevant sein, da die Implementierung durch andere Entwickler erfolgte.

Die aus unterschiedlicher Herangehensweise potenziell resultierende Komplexität ist erst für die Beantwortung der Frage, ob eine geistige Schöpfung vorliegt, von Relevanz.

Der Quellcode enthält in der Regel auch in seiner simpelsten Form eine Folge von aufgabenspezifischen Befehlen an eine Maschine, sei es der Import der notwendigen Frameworks (vgl. Abbildung 7.1 Zeilen 2 und 3), die Initialisierung eines Netzwerk-Objektes (vgl. Abbildung 7.1 Zeile 10) oder das Laden von Dateiinhalten.

Ein Computerprogramm im Sinne des § 69a Abs. 1 UrhG dürfte mithin im Rahmen des Quellcodes grundsätzlich gegeben sein.

344 In der Praxis müsste das Modell noch für den Einsatz in einer Anwendung vorbereitet werden - die Bereitstellung könnte z. B. über Tensor Flow Serving erfolgen, dafür würde das Modell noch mittels der Tensor Flow-Methoden vorbereitet, vgl. z. B. Ausführungen hier https://towardsdatascience.com/deploying-keras-models-usingtensorflow-serving-and-flask-508ba00f1037 (Stand: 22.02.2021). 
bb) Hyperparameter und Parameter

Schon dem Begriff nach fällt es schwer, Daten, Datenbanken und Dateistrukturen - und damit also auch Hyperparameter - als Computerprogramme im Sinne des $\S 69$ a UrhG aufzufassen, zumal sie auch in den $\S \S 4$ und 87a UrhG implizit aus dem Schutz ausgenommen werden. ${ }^{345}$ Jedoch wird auch Quellcode in Dateien gespeichert, und auch kompilierter Code kann in Dateiform vorliegen. An dieser Stelle ist also zu klären, ob die in Dateien ausgelagerten Parameter und Hyperparameter ein Computerprogramm im Sinne des $\S 69$ a UrhG darstellen, oder doch zumindest ein Computerprogramm-Teil.

Wenngleich der Gedanke naheliegt, dass gespeicherte Parameter und $\mathrm{Hy}$ perparameter letztlich doch gespeicherten Steuerbefehlen ähneln könnten, so ist doch vernünftigerweise davon auszugehen, dass es sich lediglich um Informationen handelt, die von Steuerbefehlen verwendet werden, im Unterschied zu (kompiliertem) Code, der so, wie er vorliegt, an ein (Ausführ-) Programm übergeben und ausgeführt werden kann. Anders sieht dies Grätz, der unter Annahme eines weiten Begriffsverständnis von Computerprogrammen, das keine Steuerungsfunktion fordert, davon ausgeht, dass auch „Trainingsergebnisse“ (hier: Parameter) als Computerprogramm zu verstehen sind. ${ }^{346}$ Dem ist nicht zuzustimmen. Hierbei wird übersehen, dass Trainingsergebnisse für sich nur eine Sammlung von Werten sind, die nicht ausgeführt werden können. Selbst wenn keine Steuerungsfunktion gefordert wird, fehlt den Daten eine für Computerprogramme übliche Struktur.

An der Steuerungsfunktion aber fehlt es: Hyperparameter und Parameter sind letztendlich nur Werte, die nach dem Laden der Datei durch den Quellcode im Programm Variablen zugewiesen werden können, aber es handelt sich eben nicht um eigenständige Steuerbefehle. Damit verfängt auch das Argument nicht, dass außerhalb des Quellcodes gespeicherte Werte das Verhalten des Computers auf gleiche Weise steuern wie im Code selbst hinterlegte Werte, weshalb auch die ausgelagerten Werte genauso Teil des Computerprogramms seien. ${ }^{347}$ Die ausgelagerten Hyperparameter für sich sind faktisch nämlich nicht in der Lage, den Computer zu steuern.

345 Wandtke/Bullinger-Grützmacher, PK UrhR, § 69a Rn. 17.

346 Grätz, Künstliche Intelligenz im Urheberrecht, S. 52.; einen weiten Computerprogrammbegriff ebenso erwägend, aber den Schutz aufgrund fehlender geistiger Schöpfung ablehnend Ehinger/Stiemerling, CR 12 2018, 761, 768; das OLG Hamburg setzt ebenfalls für die Annahme eines Schutzes voraus, dass die fragliche Datei Steuerbefehle enthält, s. OLG Hamburg MMR 1999, 230, 231 - Superfun.

347 Nebel/Stiemerling, CR 1 2016, S. 66. 
Allerdings erfasst § 69a UrhG auch Entwurfsmaterial, und damit vorgelagerte Ausdrucksformen eines Programms, wie zum Beispiel Flussdiagramme oder andere Vorstufen des Computerprogramms. ${ }^{348}$ Es könnte daran gedacht werden, die im JSON- oder ähnlichen Format abgelegten Hyperparameter (nicht jedoch die errechneten bzw. optimierten Parameter) über diese Vorschrift in den Schutz einzubeziehen, stellen sie doch in gewisser Weise den „Entwurf" des trainierten Netzwerkes dar, dergestalt dass sie den Aufbau des entstehenden KNN bestimmen.

Nach den Erwägungsgründen der für die Entstehung von § 69a UrhG maßgeblichen Computerprogramm-RL muss das am Schutz teilnehmende Entwurfsmaterial zur Entwicklung (oder zur Vorbereitung) eines Computerprogramms dienen, wobei die Art der vorbereitenden Arbeit die spätere Entstehung eines Computerprogramms zulassen muss. ${ }^{349}$ Problematisch erscheint, dass die Hyperparameter nicht zur Vorbereitung auf die Entwicklung eines Programms dienen, sondern lediglich abstrakt das KNN (ohne die Gewichtungsinformationen) abbilden. Entwurfscharakter haben sie also allenfalls für das entstehende KNN, nicht aber für ein konkretes Computerprogramm. Die Subsumtion unter den Begriff „Entwurfsmaterial“ will hier demzufolge nicht so recht passen. ${ }^{350}$ Vielmehr sind sie als computeranweisungslose Daten einzuordnen, die keinen Schutz als Computerprogramm genießen. ${ }^{351}$

Weder die abgespeicherten Parameter noch die Hyperparameter sind folglich - für sich betrachtet - dem Schutz nach § 69a UrhG zugänglich.

\section{cc) Kombination zur Laufzeit}

Möglicherweise können die in Dateien ausgelagerten Informationen jedoch anderweitig in den Schutz aus $§ 69$ a UrhG einbezogen werden: Die Parameter und Hyperparameter werden aus den Dateien eingelesen und im flüchtigen Arbeitsspeicher mit den aus dem Quellcode generierten Steueranweisungen im Bytecode zusammengeführt. § 69a UrhG erfasst jede Ausdrucksform

348 Dreier/Schulze-Dreier, UrhG, § 69a Rn. 14.

349 Wandtke/Bullinger-Grützmacher, PK UrhR, § 69a Rn. 7; Erwägungsgrund 7 und Art. 1 Abs. 1 S. 2 Computerprogramm-RL.

350 Gegen einen Schutz als Entwurfsmaterial auch Hartmann/Prinz, WRP 12 2018, $1431,1435 \mathrm{f}$. .

351 Vgl. dazu Dreier/Schulze-Dreier, UrhG, § 69a Rn. 12. 
eines Computerprogramms, auch den Maschinen- und Objektcode. ${ }^{352}$ Damit könnte also unter Umständen auf das nur flüchtig existierende Endprodukt als Schutzobjekt abgestellt werden, wobei jedoch fraglich ist, ob dies eine ausreichend manifestierte Form darstellt. Grundsätzlich reicht jedoch die (auch einmalige) Wahrnehmbarkeit, eine permanente Fixierung ist nicht erforderlich. ${ }^{353}$ Die entstehende „Kombination zur Laufzeit“ ist eine andere Ausdrucksform des Computerprogramms, das bereits durch den Quellcode geschützt ist. Der entstehende Bytecode ist also, sofern er überhaupt außerhalb des Programmablaufs erreichbar ist - ebenso wie bereits der Quellcode Computerprogramm im Sinne des § 69a Abs. 1 UrhG, jedoch nicht zusätzlich zum Quellcode, sondern in dessen Rahmen geschützt.

dd) Sonstige Schutzgegenstände

In Bezug auf KNN wird häufig auch ,der Algorithmus“ als Schutzgegenstand diskutiert, ${ }^{354}$ wobei es wesentlich darauf ankommt, was als ,der Algorithmus“ verstanden wird. ${ }^{355}$ Der „Algorithmus“ im Sinne des aus dem Quellcode entstehenden Programms wurde bereits abgehandelt. ${ }^{356}$ Es ist möglich, das Verständnis eines KNN auf die zugrundeliegenden statistischen Rechenregeln zu reduzieren. Diese wären sodann als Algorithmus im mathematischen Sinne aufzufassen und als Ideen und Grundsätze gem. § 69a Abs. 2 UrhG nicht schutzfähig. Die hier verwendete Definition eines KNN rekurriert jedoch nicht auf diese abstrakten Rechenregeln, sondern auf deren konkreter Umsetzung durch die in $\S 6$ D.III. erläuterten Bestandteile eines trainierten Modells, sodass es allein auf deren Schutzfähigkeit ankommt. Eine Diskussion der Schutzfähigkeit des Algorithmus als gesonderter Schutzgegenstand ist also entbehrlich.

352 Wandtke/Bullinger-Grützmacher, PK UrhR, § 69a Rn. 11; Dreier/Schulze-Dreier, UrhG, § 69a Rn. 19.

353 Wandtke/Bullinger-Grützmacher, PK UrhR, § 69a Rn. 11.

354 Vgl. z. B. Hauck/Cevc, ZGE 11 2019, 135, 160; Linke, GRUR Junge Wissenschaft 2019, S. 36 ff., S. 40 f..

355 Zur begrifflichen Ambivalenz vgl. Dreier/Schulze-Dreier, UrhG, § 69a Rn. 22; zur Einordnung in § 69a UrhG vgl. Söbbing, CR 42020.

$356 \mathrm{Vgl}$. oben aa) und cc). 
ee) Zusammenfassung: infrage kommende Schutzgegenstände

Für den Schutz als Computerprogramm kommt folglich nur der das Modell ladende und ausführende Quellcode, sowie damit auch der entstehende Bytecode infrage.

\section{Eigene geistige Schöpfung}

Als Schutzgegenstand wurde der das trainierte KNN ladende und ausführende Quellcode identifiziert. Für einen Schutz gem. § 69a UrhG muss diesem die Qualität eines individuellen Werkes im Sinne eines Ergebnisses einer eigenen geistigen Schöpfung des Urhebers zukommen (§ 69a Abs. 3 S. 1 UrhG). Auffällig ist hier, dass nicht - wie etwa in $\S 2$ Abs. 2 UrhG - eine „persönliche“, sondern eine ,eigene“ geistige Schöpfung gefordert wird. Dies ist jedoch der Umsetzung der Computerprogramm-RL, und damit der Absenkung der vormals noch deutlich höheren Schutzvoraussetzungen, ${ }^{357}$ geschuldet, ${ }^{358}$ es besteht Einigkeit darüber, dass - unabhängig vom Wortlaut in $§ 69$ a Abs. 3 S. 1 UrhG - eine individuelle persönliche geistige - menschliche - Schöpfung im Sinne des $§ 2$ Abs. 2 UrhG erforderlich ist. ${ }^{359}$ Folglich ist auch hier eine menschlich-gestalterische Tätigkeit erforderlich, die ,einen geistigen Gehalt aufweist, zu einer Formgestaltung geführt hat und eine hinreichende Individualität erkennen lässt" $“ 360$

\section{a) Menschlich-gestalterische Tätigkeit}

Das Kriterium der menschlich-gestalterischen Tätigkeit dürfte - sofern nicht modellprogrammerzeugende Algorithmen eingesetzt werden - unproblematisch für den Quellcode-Anteil trainierter Modelle vorliegen. Dieser wird auch nicht automatisiert geändert, die automatisierten Einfügungen der Parameterwerte und Hyperparameter erfolgen zum einen durch den Programmierer initiiert, und zum anderen erst bei Programmablauf. Abzustellen ist aber auf den Zeitpunkt der Herstellung des Quellcodes.

357 BGH GRUR 1985, 1041, 1047 - Inkasso-Programm

358 Dreier/Schulze-Dreier, UrhG, § 69a Rn. 25.

359 Dreier/Schulze-Dreier, UrhG, § 69a Rn. 25; Nordemann/Fromm-Czychowski, UrhR, § 69a Rn. 16; Ahlberg/Götting-Kaboth/Spies, BeckOK-UrhG, § 69a Rn. 13.

360 Dreier/Schulze-Dreier, UrhG, § 69a Rn. 26. 
Ferner müsste der Quellcode einen geistigen Gehalt aufweisen, zu einer Formgestaltung geführt haben und eine hinreichende Individualität erkennen lassen. ${ }^{361}$

\section{b) Geistiger Gehalt}

Geistigen Gehalt weist das Computerprogramm auf, wenn in ihm der menschliche Geist zum Ausdruck kommt ${ }^{362}$ - die Idee, das Konzept, das der Programmierer oder Softwareentwickler umsetzen möchte, muss sich also in dem Quellcode wiederfinden. Im Quellcode eines trainierten KNN bedient sich der Programmierer einer Reihe verfügbarer Frameworks (sofern - wie im Regelfall - nicht der gesamte Quellcode neu geschrieben, also quasi ,das Rad neu erfunden" wird). Schon die Auswahl derselben und daran anschließend die Wahl der Objekttypen, die Art des KNN, und die Netzwerkarchitektur, also die Auswahl der Anzahl der Schichten, der Aktivierungsfunktionen etc. sind ein Abbild der Idee, mit dem der Entwickler sein Ziel zu erreichen gedenkt, und mithin Ausdruck seines Geistes.

\section{c) Wahrnehmbarkeit}

Das Computerprogramm müsste ferner als Ergebnis der schöpferischen Tätigkeit des Programmierers der Wahrnehmung durch die menschlichen Sinne zugänglich sein, ${ }^{363}$ es handelt sich bei Quellcode um niedergeschriebenen Text, der vom Menschen gelesen werden kann, und in der ausgeführten Fassung durch Interaktionsmöglichkeiten (Eingabe von zu analysierenden Daten, Ausgabe der Vorhersagen oder generierten Erzeugnisse) auch durch NichtEntwickler wahrnehmbar ist, auch dieses Merkmal liegt also unproblematisch vor.

\section{d) Individualität}

Möglicherweise scheitert die Subsumtion jedoch an der nötigen erforderlichen Individualität, also der ,eigenpersönlichen Ausnutzung des bestehenden

361 Dreier/Schulze-Dreier, UrhG, § 69a Rn. 26.

362 Dreier/Schulze-Dreier, UrhG, § 69a Rn. 26.

363 Dreier/Schulze-Dreier, UrhG, § 69a Rn. 26. 
Gestaltungsspielraumes“364. Diese Schlussfolgerung liegt nahe, wenn berücksichtigt wird, dass der Vorgang der Initialisierung eines KNN (also Quellcode 2 in Abbildung 6.2) für unterschiedlichste Anwendungszwecke sehr ähnlich bis identisch ausfallen kann.

Das wird umso deutlicher anhand eines Beispiels: Entwickler A möchte ein KNN darauf trainieren, Autos in Bildern zu erkennen, und verwendet dafür einen Datensatz mit Bildern von Autos sowie ein ML-Modell in einer bestimmten Konfiguration. Entwickler B möchte ein KNN darauf trainieren, Bäume in Bildern zu erkennen. Dafür verwendet er einen gänzlich anderen Datensatz mit Bildern von Bäumen, aber die gleiche Konfiguration des KNN. Der Quellcode für beide Modelle kann mithin identisch ausfallen, obgleich eine vollkommen andere Aufgabe erfüllt wird (und sich die trainierten Parameter auch wesentlich unterscheiden).

Möglicherweise existiert also ein „Basisbefehlssatz“, der für eine Vielzahl unterschiedlicher Aufgabenstellungen identisch und zugleich unerlässlich sein kann, insbesondere wenn die Hyperparameter in eine Datei ausgelagert wurden, und für den folglich ein Freihaltebedürfnis im Sinne der „Building Blocks" eines ML-Modells bestehen könnte.

Zugleich darf jedoch die Schwelle der Individualität nicht zu hoch angesetzt werden. ${ }^{365}$ Es verbietet sich an dieser Stelle eine pauschale Beurteilung, der Quellcode kann mitunter auch sehr individuell ausfallen. Zudem kann ein Programm, das zwar mit dem bis auf Dateipfade identischen Quellcode wie ein anderes Programm abläuft, dem aber gänzlich verschiedene Trainingsdaten und Hyperparameter zugrunde liegen, eine gänzlich andere Aufgabe oder eine gleiche Aufgabe mit höherer Präzision durchführen, sodass die Individualität auch in der Zusammenschau mit anderen Bestandteilen des Computerprogramms liegen kann.

Es wird vielmehr auf eine Einzelfallbetrachtung ankommen müssen. Nach der in $\S 7$ B.I. und $\S 7$ B.II. vorgeschlagenen Vorgehensweise (Schutz der Hyperparameter bzw. des trainierten KNN als Datenbank(werk)) dürfte es für den Entwickler, der einen „Standardquellcode“ zum Aufruf des KNN verwendet, jedoch auch nicht schädlich sein, wenn dieser nicht gem. § 69a UrhG geschützt werden kann, weil dann ein großer Teil seiner geistigen Schöpfung bereits Datenbankwerkschutz genießt. In vielen Fällen wird der Entwickler jedoch vermutlich zumindest einige Anpassungen des „Standardquellcodes“

364 Dreier/Schulze-Dreier, UrhG, § 69a Rn. 26.

365 Dies gilt grundsätzlich für den Computerprogrammschutz, vgl. BGH GRUR 2005, 860, 861 - Fash 2000; Dreier/Schulze-Dreier, UrhG, § 69a Rn. 26. 
vornehmen müssen, sodass dann - aufgrund der sehr niedrigen Schutzanforderungen - auch dafür ein Schutz nach § 69a UrhG angenommen werden kann. ${ }^{366}$

\section{Ergebnis}

Trainierte ML-Modelle sind dem Computerprogrammschutz nach § 69a UrhG zugänglich, wenn der Quellcode als zentraler Schutzgegenstand herangezogen wird.

Inwiefern der urheberrechtliche Computerprogrammschutz für ein trainiertes Modell relevant ist, hängt zu großen Teilen davon ab, ob der Quellcode selbst die zentralen Funktionalitäten enthält, oder ob ein minimaler „Standardquellcode“ verwendet wird, während die eigentliche „Magie“ des trainierten Modells in ausgelagerten Hyperparametern und Parametern liegt. In letzterem Fall dürfte dem Schutzsuchenden wohl der Datenbank- und ggf. Datenbankherstellerschutz besser dienen; ${ }^{367}$ wenn jedoch eine wesentliche Leistung des Entwicklers in der Implementierung des Quellcodes besteht, schützt der Computerprogrammschutz vor der Übernahme insbesondere der Gesamtstruktur und auch von (für sich als schutzfähig zu befindenden) Programmteilen. ${ }^{368}$

\section{Zusammenfassung}

Trainierte ML-Modelle sind durch die verschiedenen infrage kommenden Schutzgegenstände in unterschiedlicher Weise urheberrechtlich schutzfähig. Sofern der Fokus der Betrachtung auf dem Quellcode liegt, kommt ein Computerprogrammschutz nach § 69a UrhG infrage. Für die gewählten und gespeicherten Hyperparameter und Parameter kommt in der Kombination außerdem Datenbankwerkschutz gem. § 4 Abs. 2, Abs. 1 UrhG sowie das sui-generis Datenbankherstellerrecht gem. §§ 87a ff. UrhG in Betracht.

366 Einen Computerprogrammschutz nur im Einzelfall für möglich haltend Apel/ Kaulartz, RDi Nr.1 2020, 24, 28.

367 Vgl. dazu § 7 B.I. und § 7 B.II..

368 Vgl. Dreier/Schulze-Dreier, UrhG, § 69a Rn. 21, 23. 

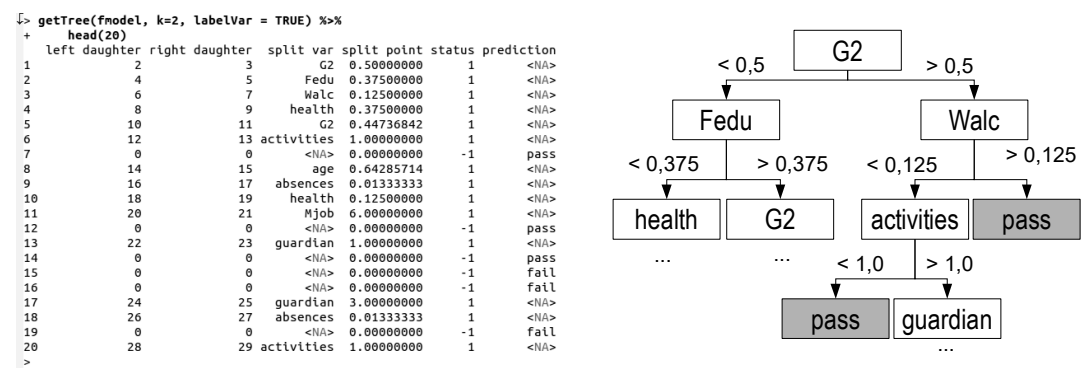

Abbildung 7.2: Die ersten 20 Einträge eines Baumes in einem Random Forest in Tabellen- und Baumform, Quelle: eigene Darstellung.

\section{Trainierter Random Forest in $\mathrm{R}$}

Das Konzept der Random Forests wurde bereits in $\S 2$ B.II.1. eingeführt, hier folgt eine Veranschaulichung anhand eines Beispiels in der Programmiersprache $R$, um die urheberrechtliche Analyse und insbesondere die sich von den dargestellten Python-Modellen unterscheidende Behandlung besser nachvollziehen zu können.

Abbildung 7.2 zeigt in tabellarischer Darstellung einen „Baum“ aus einem Random Forest, der auf einem Trainingsdatenset über portugiesische Schüler $^{369}$ und deren Erfolgsquote im Schuljahr trainiert wurde. ${ }^{370}$ Anhand dieser Abbildung kann die Funktionsweise eines Entscheidungsbaums nachvollzogen werden: Aufgabe des Modells ist es, für einen Studierenden vorherzusagen, ob er die Jahresabschlussklausur bestehen wird oder nicht. Der Datensatz enthält Informationen unter anderem über die Schule, das Geschlecht, das Alter, die Adresse, die Berufe der Eltern, bisheriges Nichtbestehen von Klausuren, Anfahrtszeiten zur Schule und Freizeitaktivitäten.

Das Modell wurde in der Programmiersprache $R$ entwickelt. Die Tabelle zeigt aus Praktikabilitätsgründen hier nur die ersten 20 Zeilen des Baumes an und ist wie folgt zu lesen:

369 Datensatz: https://archive.ics.uci.edu/ml/datasets/Student+Performance (Stand: 22.02.2021); Carvalho Brito, 15th European Concurrent Engineering Conference 2008, ECEC '2008 [and] 5th Future Business Technology Conference, FUBUTEC '2008: April 9 - 11, 2008, Porto, Portugal.

370 Beispiel entwickelt anhand von https://www.machinegurning.com/rstats/deploying models/ (Stand: 22.02.2021). 
Der oberste Knoten (1) hat zwei Kindknoten (,left daughter“ = Knoten Nr. 2 und ,right daughter“ $=$ Knoten Nr. 3). Die Eigenschaft der untersuchten Schüler, die im ersten Knoten überprüft wird (,split var“), ist „G2“ - die in Prozent gemessene Note des zweiten Leistungsmessungszeitraums. Wenn die erreichte Note über 50 Prozent (0.5) lag, wird zum Knoten 2 gesprungen, es geht dann also in Zeile 2 weiter, ansonsten zu Knoten 3. Knoten 2 hat die Kindknoten 4 und 5, und überprüft auf das Kriterium der Ausbildung des Vaters („Fedu“). Knoten 3 hat die Kindknoten 6 und 7, und überprüft, wieviel Alkohol der Schüler am Wochenende konsumiert (,Walc“). Für einen Alkoholkonsumwert unter 0.125 geht es bei Knoten 7 weiter, bei dem hier zum ersten Mal in der Spalte ,prediction“ ein Wert angegeben ist: Der Schüler hätte also bei der Kombination gute zweite Klausur (G2) und wenig Alkoholkonsum am Wochenende vermutlich die Schuljahresendklausur bestanden. ${ }^{371}$

Die Tabelle in Abbildung 7.2 zeigt, dass in einem Random Forest-Modell bzw. für Entscheidungsbäume im Allgemeinen - die trainierte Baumstruktur bereits ausreicht, um Vorhersagen für neue Daten treffen zu können. Die Tabellen aller Bäume in einem Random Forest können dann in eine Tabelle zusammengefasst und für Vorhersagen verwendet werden. Ein erneutes Einlesen in ein Programm in der Ausgangsprogrammiersprache ist nicht erforderlich, die Informationen können schlicht in einer „klassischen“ Datenbank gespeichert und mit einem beliebigen Computerprogrammcode, der die Tabelle versteht, ausgelesen werden - dabei ist dies nicht einmal zwingend erforderlich, sondern reduziert lediglich den zeitlichen Aufwand für den Menschen. Insofern gilt für ein Random Forest-Modell in $R-\mathrm{im}$ Unterschied zu Python-Modellen - dass ein trainiertes Modell und die trainierten Parameter nicht auseinanderfallen. Eine separate Prüfung erübrigt sich deshalb, es gelten die Aussagen, die diesbezüglich sogleich zum Schutz trainierter Parameter getroffen werden. ${ }^{372}$ Die Prüfung erfolgt zusammengefasst mit den trainierten Parametern der Python-Modelle, um hier den Kontrast besser herauszustellen.

371 Die Split-Werte sind nicht als absolute Werte zu lesen (so ergibt der „Alkoholkonsumwert 0.125 “ ohne weitere Informationen keinen Sinn): Für die Berechnung und Optimierung der Split-Werte werden die in allen betrachteten Datensätzen gefundenen Werte berücksichtigt, der größte Wert entspricht dann 1.0 und der kleinste Wert 0.0, alle anderen Werte liegen proportional dazwischen. So könnte etwa ein Konsum von 2 Liter Alkohol am Wochenende dem Wert 1.0 entsprechen, 0 Liter würde dann der Wert 0.0 zugeordnet.

372 Vgl. § 7 D.. 
Insbesondere gibt es zudem keinen Quellcode, der zwangsläufig Bestandteil des trainierten Random Forest-Modells in $R$ ist. Dessen ungeachtet kann in der Anwendung des trainierten Modells ein schutzfähiger Quellcode zum Einsatz kommen, und auch für den Quellcode, mithilfe dessen der trainierte Random Forest erzeugt wird, kommt ein Schutz nach § 69a UrhG infrage.

\section{Trainierte Parameter}

Bereits geklärt wurde die urheberrechtliche Schutzfähigkeit trainierter $P y$ thon-ML-Modelle, für die die Parameter jeweils nur ein Element des zu schützenden Werkes darstellen. Für Random Forests in R wurde festgestellt, dass das trainierte Modell (also die entstehende Tabelle) den trainierten Parametern entspricht. Für die Prüfung der Schutzfähigkeit der Tabelle wurde insofern auf die Prüfung trainierter Parameter verwiesen.

In einem nächsten Schritt ist also zu klären, ob auch die trainierten Parameter schutzfähig sind. Zum einen ist dabei zwischen trainierten Parametern, die nur einen Teil eines trainierten Modells in Python ausmachen, und trainierten Parametern, die das gesamte trainierte Random Forest-Modell in $R$ darstellen, zu unterscheiden ist, zum anderen bestehen aber auch strukturelle Unterschiede zwischen Random Forest-Modellen und künstlichen neuronalen Netzen, die sich etwa auf den Datenbankcharakter auswirken könnten.

\section{Schutz als Datenbankwerk gem. § 4 Abs. 2 UrhG}

Für einen Schutz als Datenbankwerk müsste die Ansammlung von trainierten Parametern gem. § 4 Abs. 2 UrhG i. V. m. § 4 Abs. 1 UrhG einem Sammelwerk entsprechen, dessen Elemente systematisch oder methodisch angeordnet und einzeln mit Hilfe elektronischer Mittel oder auf andere Weise zugänglich sind, und das aufgrund der Anordnung oder Auswahl der Elemente eine geistige Schöpfung darstellt.

\section{Datenbankwerk}

An dieser Stelle wird nicht kategorisch zwischen ML-Modellen in Python und Random Forests in $R$ unterschieden, sondern zwischen KNN und Ran- 
dom Forests an sich, da die Unterschiede der Modelle in Bezug auf die trainierbaren Parameter hier besonders hervortreten.

\section{a) Künstliches neuronales Netz}

Elemente der Sammlung trainierter Parameter eines KNN sind die einzelnen Werte, die indizieren, mit welcher Gewichtung der Output eines Neurons an das nächste Neuron weitergeleitet wird. Der Abruf eines einzelnen Wertes eröffnet darüber hinaus auch Informationen über die Position im Netzwerk, für die dieser Wert gilt (diese Information muss zwangsläufig enthalten sein, denn ansonsten könnten die Werte nicht wieder eingelesen werden, um das Training fortzusetzen bzw. das trainierte Netz produktiv zu verwenden). Analog zur Postleitzahl ${ }^{373}$ könnte dadurch auch dem einzelnen trainierten Parameter ein Informationswert zuzusprechen sein. ${ }^{374}$ Die methodische bzw. systematische Anordnung und die einzelne Zugänglichkeit, die schon für das Zusammenspiel zwischen abgespeicherten Parametern und Quellcode unerlässlich sind, liegen ebenfalls vor. Mithin sind nicht nur die Kriterien der systematischen bzw. methodischen Anordnung sowie der einzelnen Zugänglichkeit, sondern insbesondere aufgrund ihres jeweils vorhandenen eigenen Informationswerts auch das der Unabhängigkeit der Elemente erfüllt.

\section{b) Random Forest}

Elemente eines trainierten Random Forest-Modells könnten die einzelnen Bäume oder - eine Ebene tiefer - die Knoten samt ihrer Schwellwerte und Feature-Information darstellen. Der Knoten als Element gibt also Auskunft über ein in dem zugehörigen Baum untersuchtes Feature sowie den ermittelten Schwellwert (im Beispiel der portugiesischen Schüler: untersuchtes Feature könnte die am Wochenende konsumierte Alkoholmenge sein, der Schwellwert ist dann die auf einen Wert zwischen 0 und 1 skalierte Alkoholmenge, die im weiteren Verlauf voraussichtlich zu einem Nichtbestehen führt). Die Elemente sind folglich unabhängig informierend. Die Sammlung erfolgt zwangsläufig strukturiert, und die Elemente sind einzeln abrufbar.

373 Vgl. wieder Nordemann/Fromm-Czychowski, UrhR, § 4 Rn. 26; b).

374 A.A. Hartmann/Prinz, WRP 12 2018, Rn. 62. 
c) Zwischenergebnis

Das Vorliegen einer Datenbank im Sinne des $\S 4$ Abs. 2 UrhG kann also sowohl für Random Forests als auch für KNN angenommen werden.

\section{Persönliche geistige Schöpfung}

Es bleibt zu klären, ob jeweils auch eine persönliche geistige Schöpfung im Sinne des $\S 4$ Abs. 1 UrhG vorliegt. An dieser Stelle genügt die Differenzierung zwischen KNN und Random Forest nicht mehr, sondern es ist anhand der verwendeten Technologie zu unterscheiden: Bei der Entwicklung von Random Forests in $R$ nimmt die Parametersammlung eine andere Form an als in Python, jedoch können auch in Python Random Forests trainiert werden. Die weitere Prüfung unterscheidet daher zwischen ML-Modellen in Python und Random Forests in $R$.

\section{a) ML-Modell in Python}

In Python werden die betreffenden Parameter-Werte unzweifelhaft durch einen Computer erzeugt bzw. errechnet. ${ }^{375}$ Anknüpfungspunkt für die persönliche geistige Schöpfung ist die Anordnung oder Auswahl der Elemente. Auch die Anordnung der Parameter wird vom Framework vorgegeben und durch den Computer vorgenommen, ohne dass der Mensch einzugreifen braucht. Auf die Auswahl hat der Mensch allenfalls beschränkten Einfluss durch die Auswahl der Hyperparameter und indem er bestimmt, wann er den Trainingsprozess für abgeschlossen erklärt (denn erst dann verändern sich die Parameter nicht mehr). In keinem Fall jedoch wählt der Mensch die Parameter gezielt selbst aus.

Die Idee, die der Entwickler mit dem KNN umsetzen möchte, ist ferner nicht (allein) in der Parametersammlung verkörpert. Das beabsichtigte Ergebnis kann beim Einsatz der vorgestellten Python-Frameworks erst im Zusammenwirken mit den ausgewählten Hyperparametern erzielt werden. Die trainierten Parameter sind - auch als Sammlung - für sich genommen also keine persönliche geistige Schöpfung des Entwicklers, sondern lediglich ein

375 Vgl. zur Berechnung im Rahmen des Trainingsvorgangs z. B. oben § 3 A.II.. 
Bestandteil einer umfassenderen Schöpfung, ohne selbst dieses Erfordernis zu erfüllen.

\section{b) Random Forest in $R$}

Eine differenzierte Betrachtung ist in Bezug auf Random Forest-Modelle in $R$ geboten. Es besteht die Möglichkeit, das Ergebnis eines Trainingsvorgangs - also „den Random Forest“ - in einer „klassischen“ elektronischen Datenbank zu speichern und - ohne eine erneute Initialisierung eines Random Forest-Objektes in der ursprünglichen Programmiersprache - damit bereits Vorhersagen zu treffen. Mitunter entsprechen also die trainierbaren Parameter eines Random Forest-Modells dem trainierten Modell. Allerdings werden die Bäume und Knoten ebenfalls durch den Computer ermittelt. Die vom Entwickler gewählten Hyperparameter haben lediglich begrenzende Wirkung (bspw. kann eine maximale Baumtiefe vorgegeben werden). Der Entwickler überlässt es aber dem Algorithmus, die optimale Konfiguration zu finden. Die einzige Auswahlleistung des Menschen besteht erneut darin, zu bestimmen, wann das computergenerierte Ergebnis ausreichend den eigenen Vorstellungen entspricht. Aber auch hierbei wählt der Entwickler nicht gezielt Parameter, sondern bestimmt in der Regel anhand einer Metrik (wie zum Beispiel durch Anwendung einer Verlustfunktion, oder andere gängige, in der Regel nicht selbst entwickelte sondern in Entwicklerkreisen bewährte Funktionen, die die Abweichung der Vorhersage von der aufgrund der Labels erwarteten Vorhersage berechnen), wie nah das Ergebnis an das Optimum heranreicht, und lässt das Modell die Parameter so lange optimieren, bis ihm das Gesamtergebnis genau genug erscheint.

Fraglich könnte aber sein, ob der Computer von einem Menschen steuernd als Hilfsmittel bzw. Werkzeug zur Erzeugung der Struktur eingesetzt wurde. In diesem Fall könnte eine persönliche geistige Schöpfung bejaht werden, denn dann könnte eine menschlich-gestalterische Handlung des Entwicklers vorliegen. ${ }^{376}$ Wenn etwa der Entwickler alle Rechenschritte, die der Computer durchführen soll, vorgibt, und diese dann lediglich automatisiert ausgeführt werden, könnte von einem Einsatz als Werkzeug gesprochen zu sprechen sein. Zwar gibt es bei Random Forest-Modellen auch eine Zufallskomponente, auf die der Entwickler keinen Einfluss hat: So werden etwa die Features

376 Schricker/Loewenheim-Loewenheim, UrhR, Rn. 39f., Dreier/Schulze-Schulze, UrhG, $§ 2$ Rn. 8 . 
der einzelnen Bäume durch den Algorithmus zufällig ausgewählt, ebenso die Datensätze, die die Bäume durchlaufen. Sowohl die Features als auch die Datensätze haben einen maßgeblichen Einfluss auf bzw. bestimmen die berechneten Knotenwerte, sodass die Leistung des Entwicklers vermeintlich verblasst. Jedoch legt der Entwickler - durch Anwendung besagter Metriken, und aus eigener Erfahrung - fest, wann das trainierte Modell seinen Vorstellungen entspricht, und gibt damit zum Ausdruck, dass in dem Ergebnis, also dem trainierten Random Forest-Modell in Form der durch das als Werkzeug eingesetztes Computerprogramm ausgewählten Werten in der Tabelle, seine persönliche geistige Schöpfung zum Ausdruck kommt. Die Prüfung gestaltet sich insofern ähnlich der Prüfung, die später für Erzeugnisse von ML-Modellen vorzunehmen sein wird: ${ }^{377}$ Der Entwickler wählt - für die Auswahl der Elemente seiner Sammlung, um die es hier als Bezugspunkt der geistigen Schöpfung geht - ein Framework aus, setzt begrenzende Parameter, und wählt aus mehreren Ergebnissen eines aus. Dies ist für trainierte Random Forests in $R$ aus den genannten Gründen der Fall, im Unterschied zu trainierten Parametern eines Modells in Python, die nur einen Teil des Ergebnisses darstellen.

Folglich liegt in der Auswahl der Elemente der Sammlung „trainierte Random Forests in $R$ " eine persönliche geistige Schöpfung des Entwicklers bzw. Data Scientists.

\section{Ergebnis}

Im Ergebnis ist zu differenzieren zwischen trainierten Parametern der MLModelle in Python und den Ergebnistabellen trainierter Random ForestModelle in $R$. Während für erstere der Schutz spätestens am Vorliegen einer persönlichen geistigen Schöpfung scheitert, kann eine solche für Random Forests in $R$ angenommen werden, mit der Folge des urheberrechtlichen Schutzes der Tabellen als Datenbankwerk nach $\S 4$ Abs. 2 UrhG i. V. m. § 4 Abs. 1 UrhG.

377 S. unten d); dort in Anlehnung an Dreier, FS Kitagawa, S. 881. 
II. Investitionsschutz gem. $\S \S 87$ a ff. UrhG

Wenngleich ein Schutz nach $\S 4$ UrhG zumindest für ML-Modelle in Python nicht in Betracht kommt, so könnte für die trainierten Parameter doch ein sui-generis-Datenbankherstellerschutz gem. §§ 87a ff. UrhG einschlägig sein. Im Gegensatz zu einem Schutz nach § 4 Abs. 2 UrhG ist hierfür gerade keine persönliche geistige Schöpfung erforderlich, sondern eine Investition in die Beschaffung, Sammlung, Überprüfung, Aufbereitung und Darbietung des Inhalts der Datenbank. ${ }^{378}$

\section{Vorliegen einer Datenbank}

Sowohl für die trainierten Parameter eines KNN als auch für die Baumstruktur eines Random Forest-Modells wurde die Datenbankqualität bereits untersucht und bejaht (vgl. § 7 D.I.1.).

\section{Investition}

Zusätzlich muss für $§ 87 \mathrm{a}$ UrhG eine im Rahmen der Beschaffung, Überprüfung oder Darstellung der Datenbankinhalte anfallende, sowie nach Art oder Umfang wesentliche Investition vorliegen ( $\$ 87$ a Abs. $1 \mathrm{~S} .1 \mathrm{UrhG}$ ). Für die Berechnung der trainierten Parameter sowie der Baumstruktur sind mitunter teure Hardwarekomponenten sowie erheblicher Zeitaufwand erforderlich (unter anderem für die Sammlung der Trainingsdaten, Rechenzeit und manuelle Optimierung der Hyperparameter). Zu prüfen ist, in wieweit sich diese Aufwände den in $\S 87$ a UrhG aufgeführten Investitionszwecken zuordnen lassen.

a) Gegenstand der Investition

Als Bezugspunkte der Investition nennt $§ 87$ a UrhG die Beschaffung, Überprüfung oder Darstellung der Datenbankinhalte. Dabei bezieht sich die $B e$ schaffung stets auf bereits bestehende Elemente, nicht auf die Erzeugung

378 Dreier/Schulze-Dreier, UrhG, § 87a Rn. 1. 
derselben. ${ }^{379}$ Dies ergibt sich auch schon aus den Erwägungsgründen zur Datenbank-RL: Es sollen Lösungen geschützt werden, die dazu beitragen, durch Informationsmanagementsysteme der wachsenden Datenmenge Herr zu werden - und nicht solche, die die Datenmenge erweitern. ${ }^{380}$ Dies bekräftigt auch der EuGH in British Horeseracing Board, wenn er das Schutzziel der Richtlinie damit beschreibt, dass durch den sui-generis-Schutz ein Anreiz dafür geschaffen werden sollte, Systeme für die Speicherung und Verarbeitung vorhandener Informationen zu errichten, und eben nicht für die Erzeugung neuer Elemente, die dann ,später in einer Datenbank zusammengestellt werden können“. ${ }^{381}$

Fraglich ist folglich, ob vorliegend neue Elemente erzeugt werden.

\section{aa) Berechnung bzw. Optimierung der Parameter}

Die berechneten Parameter bzw. die Baumstruktur könnten möglicherweise als eine kondensierte Verkörperung der relevanten Informationen und Gemeinsamkeiten aus der Gesamtmenge der Trainingsdaten (also Bilder, Texte, ...) anzusehen sein. Dann dienen gerade die berechneten bzw. optimierten Werte dazu, die aus den Massen an zur Verfügung stehenden Daten gewonnen Erkenntnisse zu fixieren, also den größtmöglichen Nutzen daraus zu ziehen, ohne dass dabei die ursprüngliche Datenmenge erweitert wird. ${ }^{382}$ Es könnte dann von einer „Beschaffung“ als tauglichem Investitionsgegenstand ausgegangen werden. Hetmank/Lauber-Rönsberg kommen zu dem Ergebnis, dass - sofern die zugrundeliegenden (Trainings-)Daten allgemein zugänglich sind - die Investition in die Analyse derselben durch ML-Modelle dem Schutz nach $\S 87$ a UrhG zugänglich sein müsste. ${ }^{383}$ Eine begriffliche Abgrenzung zwischen bereits vorhandenen und neu erzeugten Daten trifft Wiebe: „Bereits vorhandene Daten sind allgemein verfügbar und können daher grundsätzlich von jedem Dritten mit gleichem Aufwand gesammelt werden, während er-

379 EuGH GRUR 2005, 244 - British Horseracing Board, BHB-Pferdewetten; BGH GRUR 2005, 857 - Hit Bilanz; Dreier/Schulze-Dreier, UrhG, § 87a Rn. 12.

380 Vgl. ErwGr 10 Datenbank-RL.

381 EuGH GRUR 2005, 244, 247 Rn. 31 - British Horseracing Board, BHBPferdewetten.

382 So wohl auch Ahlberg/Götting-Vohwinkel, BeckOK-UrhG, § 87a Rn. 49.

383 Hetmank/Lauber-Rönsberg, GRUR 2018, 574, 578. 
zeugte Daten 'ihrer Natur nach' niemandem außer dem Datenerzeuger selbst bekannt sind.“" 384

Die in der Literatur und Rechtsprechung erkennbare Neigung, den Investitionsbegriff im Kontext des Machine Learning zunehmend weiter auszulegen, ${ }^{385}$ ist auch auf EU-Ebene nicht unbemerkt geblieben. ${ }^{386}$ In einer im Jahr 2018 veröffentlichten zweiten Evaluierung der Datenbank-RL wird explizit Bezug auf maschinengenerierte Daten genommen - wenngleich eine konkrete Erörterung zu Machine Learning leider ausbleibt. Machine-Generated Databases werden gleich zu Beginn als nach allgemeinem Verständnis vom sui-generis-Recht ausgeschlossen bezeichnet. ${ }^{387}$ Sodann wird jedoch in Auswertung einer durchgeführten Umfrage und nicht zuletzt auch im Lichte des Autobahnmaut-Urteils des $\mathrm{BGH}^{388}$ zumindest festgestellt, dass - während das Verständnis des Investitionsgegenstands weiter eng zu fassen ist - eine Beobachtung der Situation erforderlich sei. ${ }^{389}$ Im Rahmen der durchgeführten Studie wird zudem angezweifelt, ob das sui-generis-Recht bzw. die tatbestandlichen Voraussetzungen noch wirtschaftlich optimal angelegt sind. ${ }^{390}$ Wenngleich im Rahmen dieser europäischen Untersuchung also keine eindeutige Tendenz zu einem weiteren Investitionsbegriff zu erkennen ist, bieten die Erörterungen vor dem Hintergrund der gebotenen „Beobachtung der Situation" doch Anlass dazu, einen genaueren Blick auf die Parametersammlung im Machine Learning-Prozess zu werfen.

Nach dem bisher Gesagten muss es wesentlich darauf ankommen, ob die Parametersammlung als Sammlung vorbestehender Informationen oder als Zusammenstellung erzeugter Informationen einzuordnen ist.

Vielleicht ist Machine Learning grundsätzlich als Informationsextrahierungsprozess ${ }^{391}$ zu begreifen - dies implizierte, dass keine neuen Informationen bzw. Daten erzeugt, sondern lediglich vorhandene Informationen aus bestehenden Daten extrahiert und in maschinenlesbare Form gebracht wür-

384 Wiebe, GRUR 2017, 338, 341; vgl. auch Leistner, KuR 9 2007, 457, 460; zweifelnd noch im Jahr 2005 Sendrowski, GRUR 2005, 369, 372.

385 Vgl. Fußnote 384 sowie z. B. LG Köln MMR 2002, 689 - Online-Fahrplanauskunft.

386 Europäische Kommission, Evaluation DB-RL, S. 35, dort Fn. 184.

387 Dies., Evaluation DB-RL, S. 35.

388 BGH GRUR 2010, 1004 - Autobahnmaut.

389 Europäische Kommission, Evaluation DB-RL, S. 37.

390 Dies., Evaluation DB-RL, S. 40.

391 Dies legen die Definitionen des Machine Learning durchaus nahe, vgl. die Ausführungen zur Abgrenzung zu TDM in $§ 2$ B.IV.1.. 
den. ${ }^{392}$ Dann läge ein nach dem traditionellen Begriffsverständnis tauglicher Investitionsgegenstand vor. ${ }^{393}$

Ordnet man aber - wie eingangs bewusst vorsichtig im Konjunktiv angedeutet - die aus den Daten gewonnenen Informationen als in den Parametern repräsentiert ein, wird verkannt, dass die aufzudeckenden Zusammenhänge für den Fall der Python-Modelle gerade nicht nur durch die Parameter erfassbar sind, sondern dass erst im Zusammenspiel mit Hyperparametern und Quellcode bzw. Skriptaufruf eine Verkörperung dieser Erkenntnisse plausibel erscheint. Die Parameter selbst sind nur Zahlenwerte, die jeweils für sich genommen das Gewicht einer durch das Modell geleiteten Information in Bezug auf das Folgeneuron oder den Folgeknoten enthalten. Die dadurch in einem Parameter enthaltene Information (z. B. ,gewichte alle eingehenden Werte mit dem Gewicht 0,42“) entsteht erst im Laufe des Trainingsprozesses und existiert in dieser Form vorher nicht.

Insofern ist grundsätzlich davon auszugehen, dass es sich bei den Parametern allenfalls um neue, also hergestellte, Datenbankelemente handelt. Die Investition in die Erzeugung in Form der Berechnung bzw. Optimierung derselben ist folglich kein tauglicher Gegenstand des § 87a UhrG.

Noch eindeutiger ist die Situation, wenn ein ML-Modell nicht Zusammenhänge aus vorbestehenden Daten ermittelt, sondern - wie etwa im Fall von sogenannten Generative Adversarial Networks ${ }^{394}$ - aus „weißem Rauschen“ lernt, ein Bild zu erzeugen. Zwar ist der adversariale Part des Modells ein „klassisch“ trainiertes Modell, aber der generative Part optimiert seine Parameter aufgrund des Feedbacks des adversarialen Teils. Die Parameter des generativen Parts sind mithin nicht eine Repräsentation vorhandener Informationen, sondern stehen für durch das Modell im Trial-and-Error-Prozess erlerntes „Wissen“. Die gespeicherten Parameterwerte sind mithin als neue, „hergestellte“ Informationen einzuordnen.

392 Vgl. auch BGH GRUR 2005, 857, 859 - HIT BILANZ - die „Feststellung vorhandener Vorgänge“" wird als Ermittlung vorhandener Informationen eingeordnet; Schricker/Loewenheim-Vogel, Urheberrecht, § 87a Rn. 57 - Abfassung von Zeitungsartikeln könnte als Erfassung von Daten ,,aus dem Leben“ einzuordnen sein; Wiebe, GRUR 2017, 338, 341 - für einen Schutz nach § 87a UrhG, wenn lediglich „in der Natur bereits vorhandene Daten“ gesammelt werden, die ,,von jedem Dritten mit gleichem Aufwand gesammelt werden können“.

393 Dagegen aber Wandtke/Bullinger-Hermes, PK UrhR, § 87a Rn. 41, der grundsätzlich Ergebnisse Big Data-Anwendungen als „Spin-Off“-Datenbanken und damit als nicht schutzfähig einordnet.

394 Vgl. § 2 B.II.3.. 
Wie bereits angedeutet, ist die Lage für trainierte Parameter von Random Forest-Modellen, die mit $R$ erzeugt wurden, anders zu beurteilen. An dieser Stelle sei nochmals an die Abbildung 7.2 erinnert: Ergebnis des Trainingsvorgangs ist in $R$ eine Baumstruktur, die in Tabellenform vorliegen kann. Diese Tabelle kann - zwar unter je nach Tabellengröße und Baumtiefe nicht unerheblichem Aufwand - bereits dazu eingesetzt werden, Zusammenhänge aus den Trainingsdaten auszulesen. Sie dokumentiert also Gemeinsamkeiten von Daten und zeigt Informationen auf, die auch ohne Einsatz des ML-Modells - oder unter Einsatz anderer Modelle - aufgedeckt werden können. Mithin spricht vieles dafür, die darin enthaltenen Informationen als bereits ,in der Natur" (bzw. in den Trainingsdaten) vorhanden anzusehen und die entstehende Tabelle lediglich als eine Sammlung und Sichtbarmachung dieser Informationen zu verstehen. Damit kommt für solche Modelle als Investitionsgegenstand auch die Investition in die Erzeugung dieser Ergebnistabelle in Betracht. Allerdings ist hier abzuwarten, wie sich die Rechtsprechung dazu äußert - oder ob seitens der EU noch weitere Klarstellungen erfolgen.

\section{bb) Andere Investitionsgegenstände}

Möglicherweise können jedoch andere Investitionsgegenstände, die im Zusammenhang mit der Herstellung der Parameterdatenbank anfallen, berücksichtigt werden. Neben der Beschaffung sind auch die Überprüfung und die Darstellung der Daten berücksichtigungsfähig ( $\$ 87$ a Abs. 1 S. 1 UrhG). Die Parameter werden in der Regel nicht dargestellt, hier kommt also keine berücksichtigungsfähige Investition infrage. Für die Überprüfung der Parameter gilt Ähnliches wie für die Ergebnisausgabe: Die Überprüfung der Parameter außerhalb des Modells ist nicht relevant, denn erst mit den Hyperparametern können die Parameter überhaupt evaluiert werden. Insofern ist wieder auf das gesamte „trainierte Modell“ abzustellen, und nicht separat auf die Parameter. Ein anderer Investitionsgegenstand kommt also nicht in Betracht.

cc) Zwischenergebnis

Als Investitionsgegenstand kommt nur die Wissensextraktion aus den Trainingsdaten im Rahmen des Trainings von Random Forests in $R$ in Betracht. Für ML-Modelle in Python kann hier für den isolierten Schutz der Parameter kein tauglicher Investitionsgegenstand festgestellt werden. 


\section{b) Wesentlichkeit}

Ferner müsste für den Fall der Random Forests in $R$ die Investition in die Wissensextraktion - also in das Training - wesentlich sein. Es dürfte sich also nicht um eine „Allerweltsinvestition“ handeln. Hier gilt im wesentlichen das Gleiche wie schon für die Wesentlichkeit der Investition in trainierte KNN festgestellt wurde: ${ }^{395}$ „Es kommt darauf an“, aber für tiefe und aufwendige Modelle ist von der Wesentlichkeit der Investition auszugehen.

\section{Ergebnis}

Zumindest für trainierte Random Forests in $R$ ist ein Schutz im Rahmen des Datenbankherstellerrechtes gem. $\S \S 87 \mathrm{a}$ ff. UrhG denkbar, während der Schutz für trainierte Parameter in ML-Modellen, die unter Einsatz der beschriebenen Python-Bibliotheken bzw. Frameworks optimiert bzw. erzeugt wurden, nicht infrage kommt.

\section{Bewertung und praktische Relevanz}

In der Praxis hat die Übernahme nur der Parameter eines Modells in Python, sei es KNN oder Random Forest, keine Relevanz: ohne die dazugehörigen Hyperparameter sind die Informationen nutzlos.

Anders sieht es aus für Random Forest-Baumstrukturen in $R$ : Diese sind auch ohne Hyperparameter mittels Datenbankabfragen für Vorhersagen nutzbar, sodass ein Schutzbedarf für die reinen trainierten Parameter besteht. Die Rechte, die dem Datenbankherstelle ${ }^{396}$ gem. $\$ 87 \mathrm{~b}$ UrhG zustehen, umfassen nach $\S 87 b$ Abs. 1 S. 1 UrhG das ausschließliche Recht zu Vervielfältigung, Verbreitung und öffentlichen Wiedergabe der gesamten Datenbank oder eines wesentlichen Teils davon. In Bezug auf die Verwendung unwesentlicher Teile der Datenbank gesteht $\S 87 b$ Abs. 1 S. 2 UrhG dem Datenbankhersteller ebenfalls ausschließliche Rechte zu, wenn die Nutzung wiederholt und systematisch erfolgt und einer normalen Auswertung der Datenbank zuwiderläuft oder die berechtigten Interessen des Datenbankherstellers unzumutbar beeinträchtigt. Insbesondere der Vervielfältigungsschutz dürfte in der 
Praxis relevant sein, denn eine umfangreiche und sorgfältig optimierte Random Forest-Struktur kann zum Beispiel einem Expertensystem als wertvolle Wissensbasis dienen.

\section{Zusammenfassung}

Auch für trainierte Parameter kommt ein Schutz nach $\S 4$ Abs. 2 UrhG sowie nach $\S \S 87$ a ff. UrhG - in Betracht, sofern es sich um die trainierten Parameter eines Random Forest-Modell in einer $R$-Implementierung handelt. Mithilfe der eingangs beschriebenen Python-Frameworks bzw. Bibliotheken erzeugte Parametersammlungen können an dem Schutz jedoch nicht partizipieren.

\section{E. Hyperparameter}

Die rechtliche Lage bzgl. der trainierten Parameter und der trainierten Modelle wurde bereits geklärt. Offen ist noch, ob auch die Hyperparameter allein schutzfähig sein könnten. Ein Schutzbedürfnis besteht jedenfalls insofern, als anhand der Hyperparameter und der entsprechenden Trainingsdatenbasis die trainierten Parameter reproduziert werden können. Zudem nimmt die Bedeutung von Hyperparameterkombinationen sowie die Übertragbarkeit auf andere Anwendungskontexte zu: Es ist etwa denkbar, einzelne Schichten eines KNN zu definieren, die zum Beispiel Inputdaten in Form von Bildern besonders gut auf Kanten oder Formen untersuchen können. Selbst wenn die Hyperparameter auf einem Trainingsdatensatz A trainiert wurden, können diese mitunter in andere, umfangreichere Netzstrukturen integriert und für Trainingsdatensatz B eingesetzt werden. ${ }^{397}$ Während die trainierten Parameter zumindest im Falle künstlicher neuronaler Netze alleinstehend wenig Wert haben dürften, so muss demzufolge ein Gleiches nicht unbedingt für die Hyperparameter gelten.

Als Schutzmöglichkeiten kommen wieder der Datenbankwerkschutz sowie das Datenbankherstellerrecht in Betracht, Computerprogrammschutz wurde bereits mangels Computerprogrammqualität der Hyperparameter abgelehnt. ${ }^{398}$ Ein Schutz als Entwurfsmaterial im Rahmen des Computerprogrammschutzes für das trainierte Modell kommt im Übrigen auch nicht

397 Vgl. z. B. Goodfellow et al., Deep Learning Handbuch, S. 363.

398 Vgl. bb). 
in Betracht, weil die alleinstehende Hyperparametersammlung zwar von dem Quellcode verwendet wird, aber nicht als Vorbereitung desselben dient. Vielmehr entsteht die Hyperparametersammlung erst mit der konkreten Umsetzung des (Trainings-)Programmcodes und wird dann vom (Produktiv-) Programmcode verwendet. ${ }^{399}$

\section{Schutz als Datenbankwerk gem. § 4 Abs. 2 UrhG}

\section{Datenbank}

Die Datenbankqualität gespeicherter Hyperparameter entspricht dem oben bereits Gesagten, ${ }^{400}$ es handelt sich um eine Sammlung unabhängiger und einzeln abrufbarer Elemente in Form von Informationen zur Modellstruktur, wie etwa die Größe und Anzahl verwendeter Schichten und eingesetzter Funktionen, sowie oben auch der errechneten Parameter, die schon aus technischer Erforderlichkeit heraus systematisch angeordnet und einzeln mithilfe der Framework- bzw. Bibliotheksfunktionen abrufbar sind. Alleiniger Unterschied ist an dieser Stelle bei der Betrachtung der Hyperparameterdatenbank, dass die Parameter als Sammlungselement nicht mehr berücksichtigt werden. Dies ändert jedoch nichts daran, dass das Vorliegen einer Datenbank anzunehmen ist.

\section{Persönliche geistige Schöpfung}

Fraglich ist jedoch, ob auch ohne die trainierten Parameter eine geistige Schöpfung des Entwicklers gegeben ist, weil ohne die Parameter die Manifestierung der Idee des Urhebers unvollständig sein könnte. Auch hier muss in der Auswahl oder Anordnung der Elemente die Idee des Entwicklers Ausdruck finden. Wie schon bei den Parametern ist auch die Anordnung der Hyperparameter durch die technischen Gegebenheiten vorgegeben. Es muss also wieder auf die Auswahl der Elemente, also der einzelnen Hyperparameter, ankommen.

399 Gegen einen Schutz als Entwurfsmaterial auch Hartmann/Prinz, WRP 12 2018, 1431, 1435 Rn. 38.

400 Vgl. § 7 D.I.1. bzw. § 7 B.I.1.. 
Diese werden durch den Entwickler so gewählt, dass bei Anwendung des Modells auf die Trainingsdaten die Parameter optimal berechnet werden.

Die Idee des Entwicklers kann sich mithin auch schon in der Wahl der Hyperparameter manifestieren, die im Zusammenspiel mit den Trainingsdaten letztendlich die Parameter berechnen helfen, bevor die Parameter durch den Ablauf des Trainingsprozesses festgelegt sind. Die Hyperparameter sind insofern unabhängig von den trainierten Parametern, als sie letztlich den Grundstock für diese legen (während andersherum die Parameter vollständig von den Hyperparametern und den Trainingsdaten abhängig sind). Wichtig ist an dieser Stelle allerdings die Verbindung mit den Trainingsdaten: verschiedene Trainingsdaten können mitunter zu unterschiedlichen Parametern führen (je größer die Menge an Trainingsdaten, umso weniger relevant ist jedoch eine Veränderung derselben etwa durch Wegnahme einzelner Datenpunkte).

Wenn die isolierte Sammlung von Hyperparametern urheberrechtlich geschützt sein soll, ist jedoch - wie auch in allen anderen Bereichen des Urheberrechts - zu verhindern, dass sich ein solcher Schutz auch auf triviale und in Fachkreisen bekannte, nichtoriginelle Hyperparameterkonfigurationen erstreckt. Zwar dürfen auch nach der Datenbank-RL nicht allzu hohe Anforderungen an die Schöpfungshöhe gestellt werden, ${ }^{401}$ jedoch ist eben ,nach unten“" genug Freiraum für weitere Innovationen zu lassen. Das Schutzbedürfnis besteht insbesondere bei aufwendigen, nicht lediglich zu Lehrzwecken vereinfachten, vielschichtigen und komplexen Modellen. So ist es denkbar, ein Bilderkennungsmodell zu konzipieren, das aus hunderten Schichten besteht, die auf unterschiedlichste Weise konfiguriert sind, um etwa ein Bild auf Farbe, Muster, Körperteile, Helligkeit, Komposition etc. zu untersuchen. In solch einem Fall muss auch die Sammlung der Hyperparameter ohne die errechneten Parameter bereits als schutzfähige Sammlung qualifiziert werden.

\section{Ergebnis}

Es wird also auf eine Einzelfallentscheidung ankommen müssen, um den Schutz nach § 4 Abs. 2 UrhG für Hyperparameter zu bestimmen. Der Schutz ist jedoch nicht von vorneherein ausgeschlossen.

401 ErwGr. 16 Datenbank-RL. 
II. Investitionsschutz gem. $\S \S 87 \mathrm{a}$ ff. UrhG

Auch für Hyperparameter könnten Datenbankherstellerrechte gem. §§ 87a ff. UrhG infrage kommen.

\section{Datenbank}

Dass die Hyperparameter eine Datenbank im Sinne des § 4 Abs. 2 UrhG darstellen, wurde bereits festgestellt, ${ }^{402}$ damit liegen in der Hinsicht auch die Voraussetzungen für $\S 87 \mathrm{a}$ UrhG vor.

\section{Wesentliche Investition}

Ferner müsste eine wesentliche Investition in die Datenbank getätigt worden sein. Auch hier wird eine Einzelfallbetrachtung erforderlich: Handelt es sich um Hyperparameter, die zu Beginn des Trainings lediglich aus Erfahrungssätzen geschätzt werden, wurden weder Zeit noch Geld in ihre Sammlung gesteckt. Insofern kann keine wesentliche Investition vorliegen. Handelt es sich um die im Rahmen eines Trainingsprozesses erarbeiteten bzw. gesammelten Hyperparameter, müssen die gleichen Erkenntnisse gelten wie sie für trainierte Modelle bereits festgestellt wurden: Der Prozess kann mitunter sehr viele Ressourcen fordern. ${ }^{403}$ Es könnte beispielsweise passieren, dass ein Set an Hyperparametern in einem komplexen Modell über mehrere Tage getestet wird, woraufhin sich herausstellt, dass die Hyperparameter nicht optimal gewählt sind. Daraufhin werden die Hyperparameter angepasst und ein erneuter Trainingsvorgang wird gestartet. Dieser Prozess kann sich mehrfach wiederholen, also zeit- und kostenintensiv sein. Es handelt sich dann um wesentliche Investitionen in die Beschaffung und Überprüfung der Datenbankinhalte. Festzustellen ist zudem, dass die Hyperparameter-Sammlung nicht maschinell erzeugt, sondern jeder Wert durch den Menschen gewählt wird. Es handelt sich also um einen gem. § 87a UrhG berücksichtigungsfähigen Investitionsgegenstand. Darüber hinaus können Kosten entstehen, wenn

402 Vgl. § 7 E.I..

403 Vgl. Graf, Multitalent für Sprache (Spektrum.de vom 11.08.2020) - die Kosten für das Training von GPT-3 wurden dort etwa mit 5 Millionen Dollar beziffert, zudem werden die $\mathrm{CO} 2-$ Emissionen betont, die durch die intensive Hochleistungsrechnernutzung entstehen. 
etwa ganze Schichten aus anderen bewährten KNN übernommen werden sollen - sofern diese urheberrechtlich geschützt und nicht frei zur Verfügung gestellt worden sind, können Lizenzkosten anfallen. Damit läge eine Investition in die Beschaffung von Daten vor.

\section{Ergebnis}

Auch eine Sammlung an Hyperparametern kann folglich dem Schutz der $\S \S 87 \mathrm{a}$ ff. UrhG unterfallen.

\section{Zusammenfassung}

Für gewählte Hyperparameter kommt - unabhängig von der Implementierung in $R$ oder Python - ein Schutz gem. $\S \S 4$ Abs. 2, Abs. 1 und 87a ff. Ur$\mathrm{hG}$ in Betracht, wobei jeweils Einzelfallbetrachtungen erforderlich werden hinsichtlich des Vorliegens einer geistigen Schöpfung in Bezug auf das Datenbankwerk sowie hinsichtlich des Vorliegens einer wesentlichen Investition bzgl. § 87a UrhG.

\section{F. Untrainiertes Modell}

Zuvor wurde bereits auf den Schutz trainierter Modelle eingegangen. ${ }^{404}$ Die Frage des Schutzes ,untrainierter“ Modelle liegt also nahe. Fraglich ist hier allerdings bereits, inwiefern diese sich begrifflich von der Struktur bzw. der grundlegenden Architektur der Modelle, also der soeben diskutierten Hyperparametersammlung unterscheiden: Wenn hiermit ein bereits konfiguriertes, aber noch „leeres“ Modell ohne trainierte Parameter zum Beginn bzw. vor Beginn des Trainingsvorgangs gemeint ist, müssen die Aussagen über die Hyperparameter Anwendung finden. Es liegt nahe, das ,untrainierte Modell““ wie auch das trainierte Modell ${ }^{405}$ als eine Kombination aus Parametern, Hyperparametern und Quellcode aufzufassen, wobei die Parameter lediglich zufällig gewählte Werte sind und sowohl Parameter als auch Hyperparameter nicht notwendig bereits in Dateien abgelegt wurden. Auch das Modell selbst 


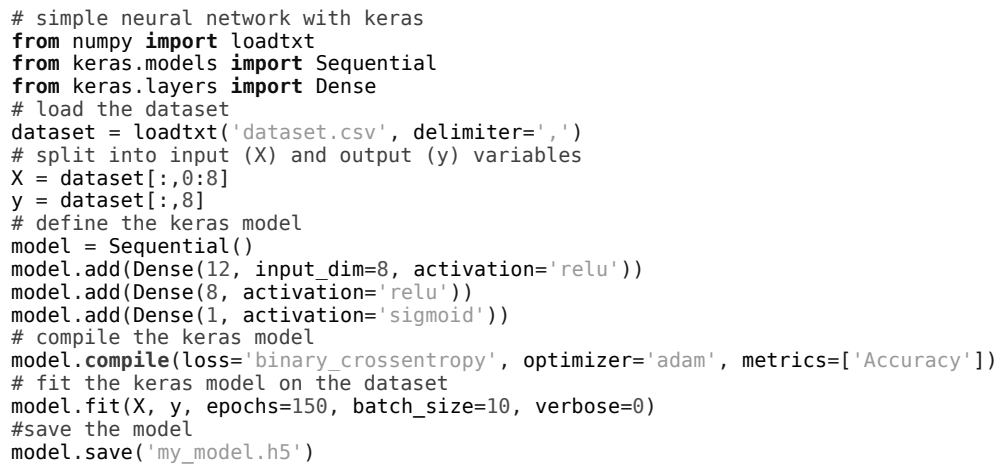

Abbildung 7.3: Beispiel eines einfachen Quellcodes zur Erstellung eines Modells mit Keras, in Anlehnung an https://machinelearningmastery.com/tutorial-first-neural-network-pythonkeras/, 22.02.2021.

liegt - im untrainierten Zustand - in der Regel nicht in Dateiform vor. Vielmehr wird das untrainierte Modell in Form von Quellcode gegeben sein, der die Modellstruktur definiert, und ggf. am Ende noch Befehle zum Trainieren und Speichern des Modells beinhaltet.

Veranschaulicht wird dies durch Abbildung 7.3. Ergebnis des ausgeführten Codes ist ein gespeichertes trainiertes Modell. Der Code selbst definiert jedoch zu Beginn ein untrainiertes Modell, außerdem liegt vor Ausführung noch kein trainiertes Modell vor, anhand dessen (z. B. durch Interaktion mit dem Nutzer) ein Einsatz etwa zur Klassifikation von Bildern erfolgen könnte. Stattdessen wird das Modell zunächst in Zeile 11 initialisiert, in den Zeilen 12 bis 14 werden die Schichten - also die Hyperparameter, bzw. die Topologie definiert. Jeder Schicht wird eine Anzahl Neuronen zugewiesen sowie die für jedes Neuron der Schicht zu verwendenden Aktivierungsfunktionen. Das Trainingsdatenset, das in Zeile 6 geladen wurde, enthält Daten (X) und Labels (y), die dann in Zeile 18 für das Training des Modells dem Modell zugeführt werden.

Als Schutzgegenstand des untrainierten Modells kommt folglich nur der Quellcode infrage, mithin also nur Computerprogrammschutz gem. § 69a UrhG. Dass im Nachhinein ggf. noch Anpassungen - auch an den Hyperparametern - vorzunehmen sind, ist unerheblich. Auch der Code für ein untrainiertes Modell erfüllt bereits einen Zweck, nämlich ein Modell zu trainieren (und 
ggf. zu evaluieren - hier nicht im Code-Beispiel enthalten) und enthält Steuerungsanweisungen. Auch die eigene geistige Schöpfung des Entwicklers nämlich, einen Weg zu finden, ein Modell für die gewählte Trainingsaufgabe programmatisch festzulegen - liegt in dem Quellcode vor. Für den Schutz gem. § 69a UrhG ergeben sich keine Unterschiede zu dem, was bereits über den Schutz des Quellcodes eines trainierten KNN gesagt wurde ${ }^{406}$ - ein Schutz als Computerprogramm ist also auch für das untrainierte Modell zumindest in Bezug auf den Quellcode regelmäßig anzunehmen.

406 Vgl. dazu oben aa). 


\section{$\S 8$ Ergebnis des dritten Teils}

Der Schutz von Modellen maschinellen Lernens ist, wie gezeigt, komplexer als auf den ersten Blick vermutet und bisher in der rechtswissenschaftlichen Literatur diskutiert. Es ist zu differenzieren zwischen den unterschiedlichen Komponenten der Modelle. ${ }^{407}$ Als potenzielle Schutzgegenstände identifiziert wurden trainierte ML-Modelle in Python, trainierte Random Forests in $R$, trainierte Parameter, Hyperparameter sowie untrainierte ML-Modelle. Diese wurden in Bezug auf ihre Schutzfähigkeit gem. $\S ~ 4,87$ a ff. und 69a UrhG untersucht.

Dabei wurde festgestellt, dass ein trainiertes Modell in Python durch die Eigenschaften seiner Komponenten (Hyperparameter- und Parametersammlung, Quellcode) potenziell Schutz sowohl als Datenbankwerk gem. $\S 4$ Abs. 2 UrhG sowie auch als Computerprogramm gem. § 69a UrhG genießt und außerdem ein Datenbankherstellerrechtsschutz gem. §§ 87a ff. UrhG infrage kommt. ${ }^{408}$

Trainierte Parameter der Random Forests unterscheiden sich bei der Implementierung in $R$ wesentlich von der Form, in der trainierte Parameter der Python-Modelle vorliegen, insofern kommt die Prüfung zu unterschiedlichen Ergebnissen: Trainierten Parametern der ML-Modelle in Python kommt weder Schutz als Datenbankwerk, noch im Rahmen des Datenbankherstellerrechtes Schutz zu (zum einen mangelt es an der persönlichen geistigen Schöpfung, zum anderen am tauglichen Investitionsgegenstand). Die trainierten Parameter der Random Forest-Modelle in $R$ stellen jedoch zugleich das trainierte Random Forest-Modell dar, weshalb sowohl eine persönliche geistige Schöpfung als auch ein tauglicher Investitionsgegenstand vorliegt. ${ }^{409}$

Isoliert abgespeicherte Hyperparameter lassen sich als Datenbank charakterisieren und dürften im Regelfall sowohl gem. § 4 UrhG als auch gem. $\S \S 87$ a ff. UrhG schutzfähig sein. ${ }^{410}$

Für untrainierte Modelle kommt nur ein Computerprogrammschutz gem. $\S 69$ a UrhG in Bezug auf den Quellcode infrage. ${ }^{411}$

407 Zur Diskussion der Modellbestandteile s. oben $\S 6$.

408 S. oben $\S 7$ B.IV..

409 S. oben $\S 7 \mathrm{D}$.

410 S. dazu $§ 7$ E..

411 S. oben $\S 7 \mathrm{~F}$. 


\begin{tabular}{|c|c|c|c|}
\hline \multirow{4}{*}{$\begin{array}{l}\text { Trainiertes Python-ML-Modell } \\
\text { Trainierter Random Forest in } R(\mathrm{RF})\end{array}$} & \multirow{2}{*}{\multicolumn{2}{|c|}{$\S \S 87 a$ ff. UrhG }} & \multirow[b]{2}{*}{$\S 69 a$ UrhG } \\
\hline & & & \\
\hline & + & + & + \\
\hline & \multicolumn{3}{|c|}{ Verweis auf trainierte Parameter } \\
\hline Trainierte Parameter & $-/+(R F)$ & $-/+(\mathrm{RF})$ & $\mathrm{n} / \mathrm{a}$ \\
\hline Hyperparameter & + & + & $\mathrm{n} / \mathrm{a}$ \\
\hline Untrainiertes Modell & $\mathrm{n} / \mathrm{a}$ & $\mathrm{n} / \mathrm{a}$ & + \\
\hline
\end{tabular}

Abbildung 8.1: Übersicht über die Ergebnisse für den Modellschutz, jeweils unter Vorbehalt einer Einzelfallbetrachtung. Quelle: eigene Darstellung.

Abbildung 8.1 stellt die Ergebnisse im Überblick dar. Ohne Schutz dürften nach dem Resultat dieser Prüfung nur gänzlich banale Modelle bleiben, ansonsten steht insbesondere der Datenbankschutz im Vordergrund. 


\section{Vierter Teil \\ Schutz von KI-Erzeugnissen}

Nachdem geklärt ist, wie es um den urheberrechtlichen Schutz maschineller Lernsysteme steht, und wie mit den dafür erforderlichen Trainingsdaten umzugehen ist, liegt eine wichtige Frage auf der Hand: Wer ist der Urheber, wenn ein ML-System ein „Werk“ „erschafft“?

Gleich zu Beginn sei gesagt: Eine einfache, und vor allem einheitliche Antwort kann hier nicht gegeben werden; dafür sind die relevanten Konstellationen und Systemarten zu verschieden.

Um einer Beantwortung immerhin näher zu kommen, sollen zunächst die Anforderungen an die persönliche geistige Schöpfung - insbesondere des menschlich-gestalterischen Handelns - in Bezug auf die Besonderheiten von Machine Learning-Modellen untersucht werden mit dem Ziel, diese Kriterien für unser Untersuchungsthema aufzubereiten. Im Anschluss daran kann ein Flussdiagramm die Beurteilung unterstützen, wer bei komplexen Machine Learning-Modellen als Urheber in Frage kommt.

$\mathrm{Zu}$ untersuchen ist also, ob das Erzeugte einem menschlichen Autor zuzurechnen ist - also ob ein Werk im urheberrechtlichen Sinne vorliegt -, und wenn ja, wem die Urheberschaft zufällt. Die Frage nach der Urheberschaft stellt sich insbesondere dann, wenn nicht eine Person alleine ein System entwickelt und einsetzt, sondern wenn mehrere Entwickler in verschiedenen Stadien sowie gegebenenfalls Nutzer an der Erstellung des Endprodukts beteiligt sind. 


\section{$\S 9$ Schutzvoraussetzungen im Urheberrecht im Kontext von KI-Erzeugnissen}

Kontext der aufgeworfenen Fragestellung ist die Prüfung der urheberrechtlichen Schutzfähigkeit beispielsweise eines Bildes oder Textes, das bzw. der den Output eines ML-Systems darstellt (im folgenden auch „KI-Erzeugnis“ oder „KI-Kunst“, ohne dabei kritisch auf die Verwendung des Kunstbegriffes einzugehen), im Rahmen des $\S 2$ UrhG. Die Prüfung erfolgt anhand der gewöhnlichen, sogleich dargestellten Kriterien und Schemata, wobei auf deren kritische Hinterfragung verzichtet werden soll, da diese keinen unmittelbar praxistauglichen Mehrwert bieten würde. Darauf wird stattdessen im Ausblick (siehe §16) eingegangen.

Die Arbeit verwendet die Begriffe „Erzeugnis“, „Output“, „Endprodukt“ und ,KI-Kunst“" weitgehend synonym, da insbesondere in diesem Kapitel nur solche Systeme im Fokus stehen, deren Erzeugnisse bei menschlicher Urheberschaft urheberrechtlich schützbare Werkarten zuzuordnen wären.

\section{A. Urheber ist stets ein Mensch}

Der erste Blick ins UrhG führt zu $§ 1$ UrhG, der besagt: „Die Urheber von Werken der Literatur, Wissenschaft und Kunst genießen für ihre Werke Schutz nach Maßgabe dieses Gesetzes." Schon im zweiten Wort des Satzes findet sich eine für den weiteren Verlauf der hiesigen Prüfung maßgebliche Anforderung: Hauptdarsteller im UrhG sind die Urheber, und diese sind nach allgemeiner Auffassung ausschließlich Menschen. ${ }^{412}$ Ein weiteres Studieren des UrhG zeigt: Die Forderung nach Menschlichkeit kann auch aus anderen Schutzvoraussetzungen herausgelesen werden. So fordert $\S 2$ Abs. 2 UrhG eine persönliche - geistige - Schöpfung: Drei weitere Hinweise darauf, dass es hier um einen menschlichen Autor gehen soll. Bezugspunkt ist also immer ein Homo Sapiens; für den Schutz zumindest nach dem Urheberrechtsgesetz de lege lata scheidet eine Maschine als Autor aus. ${ }^{413}$ In der Konsequenz ergibt

412 An Stelle vieler Dreier/Schulze-Dreier, UrhG, § 7 Rn. 2.

413 Zudem wäre fraglich, was eine Maschine mit ihr zugestandenen Urheberrechten tun würde - eine eigenständige Durchsetzung der Rechte ist nach dem heutigen Stand der Technik kaum denkbar. Vgl. zudem unten $§ 13$ D.II.. 
sich das erste Kriterium für die Prüfung: Das Werk muss einem menschlichen Urheber zurechenbar sein, andernfalls ist kein urheberrechtlicher Schutz gegeben. ${ }^{414}$

\section{B. Persönliche geistige Schöpfung}

Neben der Anforderung, dass im Sinne des UrhG Urheber nur ein Mensch sein kann, enthält $\S 2$ Abs. 2 UrhG auch die Forderung nach einer geistigen Schöpfung. Zu untersuchen ist also - zunächst abstrakt ohne Rücksichtnahme auf eine ggf. notwendige Einzelfallentscheidung für das Merkmal der Individualität - ob das Erzeugnis eines ML-Modells einer urheberrechtlichen Prüfung standhält.

Im nächsten Schritt stellt sich die Frage, welcher der unter Umständen an der Schöpfung beteiligten Personen der Schutz zuzusprechen ist, denn häufig entstehen Machine Learning-Modelle in iterativen Prozessen: Ein Grundmodell wird so lange verfeinert (nicht nur durch Training, sondern auch durch Kombination verschiedener Modelle, indem z. B. die Schichten verschiedener KNN kombiniert werden $)^{415}$ bis das gewünschte Ergebnis erzielt wird. An der Entwicklung sind in den verschiedenen Stadien mitunter unterschiedliche Entwickler beteiligt, die ihre Ergebnisse wiederum anderen zur Verfügung stellen.

Um Werkcharakter zu erlangen, genügt es also nicht, dass ein Erzeugnis vorliegt, das einer der Werkkategorien des $\S 2$ Abs. 1 UrhG zugeordnet werden kann, sondern es muss - wie alle nach dem UrhG geschützten Werke - der Forderung nach einer persönlichen geistigen Schöpfung genügen. ${ }^{416}$ Dafür sind vier wesentliche Kriterien zu prüfen:

Es muss (1.) eine persönliche Schöpfung des Urhebers vorliegen. Dies ist gegeben, wenn das Erzeugnis auf eine menschlich-gestalterische Tätigkeit des Urhebers zurückzuführen ist. ${ }^{417}$

414 DKM-Dreyer, HK-UrhG, § 2 Rn. 7; Schricker/Loewenheim-Loewenheim, UrhR, $\S 2$ Rn. 12 m. w. N.

415 Vgl. z. B. Kahou et al., Video Emotion Recognition, oder Antipov et al., Age Estimation, die jeweils verschiedene KNN-Modelle im Rahmen einer Anwendung in der Gesichtserkennung miteinander kombinieren.

416 Dreier/Schulze-Schulze, UrhG, § 2 Rn. 2.

417 Loewenheim-Loewenheim/Leistner, Handbuch Urheberrecht, § 6 Rn. 15. 
Des Weiteren (2.) muss dem Erzeugnis ein geistiger Gehalt innewohnen, das heißt, in dem Werk muss der menschliche Geist zum Ausdruck kommen, etwa durch den Ausdruck von Gedanken oder Gefühlen. ${ }^{418}$

Zudem muss die Werkschöpfung für die menschlichen Sinne wahrnehmbar geworden sein (3.), und sei es auch nur mittelbar unter Zuhilfenahme technischer Hilfsmittel. ${ }^{419}$

Abschließendes, aber zentrales Kriterium (4.) ist die Individualität des Werkes, dergestalt, dass sich das Werk ,als Ergebnis des individuellen geistigen Schaffens des Urhebers darstellt“" ${ }^{420}$ wobei an das Vorliegen der Individualität (auch „Gestaltungshöhe“ genannt) keine hohen Anforderungen zu stellen sind. Letztlich kann hierauf keine pauschale Antwort gegeben werden, sondern es ist hier eine Einzelfallentscheidung erforderlich. ${ }^{421}$

Im weiteren Verlauf wird insbesondere auf das erste Kriterium eingegangen, weil dieses die wesentliche Hürde der Zurechnung des Erzeugnisses zu einem Urheber darstellt. Auch wenn das Kriterium der Individualität in der urheberrechtlichen Prüfung als ein sehr wesentliches gilt, wird auf dieses aufgrund der starken Fallbezogenheit in dieser Arbeit nicht ausführlich eingegangen.

\section{Menschlich-gestalterische Tätigkeit}

Erste und offensichtliche Hürde im Rahmen des Einsatzes von MachineLearning-Modellen ist das Kriterium der menschlich-gestalterischen Tätigkeit, auf der das Erzeugnis beruhen muss. Es ist also der Zusammenhang zwischen Ergebnis des Modelleinsatzes und der Handlung „des Urhebers“ zu klären, wobei je nach Modellart auch fraglich sein kann, wer eigentlich der Urheber ist bzw. auf wessen Handlung abzustellen ist. ${ }^{422}$

Während der Zusammenhang zwischen der Idee eines Malers, ein Haus zu malen, und dem Ergebnis seiner Arbeit auf der Leinwand eindeutig herzustellen ist, ist es deutlich schwieriger, diesen Zusammenhang auch beim Einsatz eines zu einem gewissen Grad nicht einschätzbaren Algorithmus noch nachzuvollziehen. Konkret stellt sich etwa die Frage, ob ein Bild, das

418 Loewenheim-Loewenheim/Leistner, Handbuch Urheberrecht, § 6 Rn. 19.

419 Loewenheim-Loewenheim/Leistner, Handbuch Urheberrecht, § 6 Rn. 20.

420 Loewenheim-Loewenheim/Leistner, Handbuch Urheberrecht, § 6 Rn. 21.

421 Loewenheim-Loewenheim/Leistner, Handbuch Urheberrecht, § 6 Rn. 27.

422 Weil zum Beispiel das Tool einen Input von einem Endnutzer verlangt, und daraufhin erst ein Ergebnis produziert, vgl. Google DeepDream. 
durch einen Algorithmus ,gemalt“ wird, noch als Werk eines menschlichen Urhebers zu qualifizieren ist.

Zu klären ist also, wie der Zusammenhang zwischen der Handlung des Urhebers und der Erzeugung des „Werkes“ gestaltet sein muss, und wann dieser durchbrochen wird.

Sollte der Zusammenhang an der (vermuteten) Unberechenbarkeit eines Algorithmus scheitern, ist weiter darauf einzugehen, inwiefern Methoden zur Erklärung von Machine Learning-Modellen diesen Zusammenhang herstellen könnten.

Grundsätzlich wird diese Frage bereits seit Jahrzehnten im Kontext von Computerkunst diskutiert, ${ }^{423}$ also wenn Urheber Computer einsetzen, um Werke zu erzeugen. Der Bezug zwischen Urheber und Werk wird dabei dadurch hergestellt, dass auch beim Einsatz von Maschinen (beliebig substituierbar mit Algorithmen oder Software oder Computerprogrammen, oder letztendlich auch Tieren und Naturkräften) der Urheber eine gestalterische Tätigkeit entfaltet (bzw. entfalten muss, um den Schutz des Urheberrechtsgesetzes zu genießen), die auch in der Mitwirkung an der Erstellung des Werkes bestehen kann. ${ }^{424}$

\section{Bekanntes Terrain: Zufallskunst bzw. Computerkunst}

Dass der Einsatz eines Computers als Werkzeug für den Zurechnungszusammenhang ${ }^{425}$ zwischen der Handlung des Urhebers und der Entstehung eines Werkes beziehungsweise für das Vorliegen einer persönlichen geistigen Schöpfung nicht schädlich ist, darüber ist sind sich die Stimmen in der einschlägigen Literatur einig. ${ }^{426}$

Weniger Einigkeit besteht hingegen bei dem Einsatz von Zufallsmomenten im Rahmen des Schöpfungsprozesses. Diese Zufallsmomente können unterschiedlichste Formen annehmen: In einem Musikstück mit dem Titel „4’33“ von John Cage bestimmt die - zufällige - Geräuschkulisse im Saal

423 Vgl. z. B. Dreier, FS Kitagawa, S. 869 ff..

424 DKM-Dreyer, HK-UrhG, § 2 Rn. 28.

425 Den Zurechnungszusammenhang als ,erforderliches Bindeglied zwischen Handlung und Werkerfolg“" ausführlich diskutierend Specht-Riemenschneider, FS Taeger, 711, $717 \mathrm{f}$.

426 Vgl. u. a. Dreier/Schulze-Schulze, UrhG, § 2 Rn. 8; Ahlberg/Götting-Ahlberg, BeckOK-UrhG, § 2 Rn. 55; Wandtke/Bullinger-Bullinger, PK UrhR, § 2 Rn. 16; Möhring/Nicolini-Ahlberg, Urheberrecht, § 2 Rn. 51. 
die Gestaltung des Stücks, während der Pianist tatenlos an seinem Instrument verharrt. ${ }^{427}$ Denkbar ist auch der Einsatz zufälliger Ereignisse, wie etwa das Gießen von heißem Blei in kaltes Wasser ${ }^{428}$ oder der Einsatz sogenannter Zufallsgeneratoren ${ }^{429}$ zur Auswahl von Farben, die für ein Gemälde oder ein Kirchenfenster ${ }^{430}$ verwendet werden sollen, oder zur Auswahl von Tönen für ein Musikwerk.

\section{a) Zurechnungszusammenhang}

Wenn der gesamte Schöpfungsvorgang vom Zufall beherrscht wird, bestehen Zweifel ob der berechtigten Bezeichnung als solchen. Sofern eine Leistung auf Zufall beruht, sei sie kein „Ergebnis menschlich-gestalterischen Schaffens ${ }^{* 431}$ und damit urheberrechtlich nicht geschützt ${ }^{432}$. Der Urheber begebe sich also seiner künstlerischen Entscheidungsmacht und mache die Gestaltungsentscheidungen vom Zufall abhängig.

So soll etwa auch kein Urheberrechtsschutz erwachsen, wenn einem Computer die Auswahl und Präsentation von Tönen und Klangfarben eines Musikstücks überlassen wird. ${ }^{433}$ Es reiche nicht aus, dass die Initiative und die Idee dieser Art der Werkerstellung von einem Menschen ausgeht. ${ }^{434}$ Für das Beispiel der Computermusik sei die Lage anders zu beurteilen, wenn der Mensch die Auswahl, aus der durch den Computer zufällig gewählt wird, stark beschränkt. ${ }^{435}$ Für den Fall, dass der Computer zufällig verschiedene Ergebnisse erzeugt, und der Mensch dann für sich ein „Werk“ aus den Ergebnissen auswählt, gehen die Ansichten auseinander in Bezug auf die urheberrechtliche Schutzfähigkeit:

427 Loewenheim-Czychowski, Handbuch Urheberrecht, § 9 Rn. 104.

428 Möhring/Nicolini-Ahlberg, Urheberrecht, § 2 Rn. 50.

429 Der Name kann täuschen: Es wird kein Zufall im Sinne eines zufälligen Ereignisses generiert. Stattdessen werden in der Regel Zahlen zufällig gewählt, und anhand der zufällig gewählten Zahlen Entscheidungen quasi-zufällig getroffen.

430 Beispielsweise das Südquerhausfenster des Kölner Doms, das von Gerhard Richter gestaltet wurde - vgl. urheberrechtliche Ausführen dazu bei Lauber-Rönsberg, GRUR 2019, 244, 248.

431 DKM-Dreyer, HK-UrhG, § 22 Rn. 26.

432 Wandtke/Bullinger-Bullinger, PK UrhR, § 2 Rn. 15.

433 DKM-Dreyer, HK-UrhG, § 2 Rn. 27.

434 DKM-Dreyer, HK-UrhG, § 2 Rn. 28.

435 DKM-Dreyer, HK-UrhG, § 2 Rn. 29. 


\section{b) Auswahltheorie in Abgrenzung zur Präsentationslehre}

Die einen lassen es genügen, dass der Mensch aus den erzeugten Produkten eine Auswahl trifft und damit ein Werk als solches bestimmt (hier „Auswahltheorie" genannt), ${ }^{436}$ die anderen bemängeln an einer solchen Vorgehensweise, dass dabei gerade nicht das Ergebnis bzw. das „Werk“ auf der menschlich-gestalterischen Tätigkeit beruhe, sondern der Mensch das Ergebnis als Werk bestimmt, also ähnlich der Grundgedanken der Präsentationslehre von Kummer $^{437}$ dem Werk seinen Werkcharakter subjektiv zuweist. ${ }^{438}$ Dabei wird jedoch übersehen, dass die Vertreter der Auswahltheorie verlangen, dass der Urheber die Auswahl aus einer Reihe von Erzeugnissen trifft, deren Herstellung er kausal veranlasst hat. Hier wird auch die Abgrenzung zur Präsentationslehre deutlich: Der Urheber entdeckt nicht zufällig in der Natur einen Gegenstand, und kürt diesen dann zum Werk, sondern er veranlasst sine qua non dessen Entstehung. Zu berücksichtigen ist zudem, dass auch schon die Entscheidung, Zufallsmomente einzusetzen, gestalterisch sein kann. ${ }^{439}$

\section{c) Strenge Auswahltheorie}

Sinnvoll erscheint die Forderung von Dreier, der als Untergrenze für die Möglichkeit der Anerkennung eines urheberrechtlichen Schutzes (bereits 1992) fordert, dass der Programmierer oder der Benutzer wenigstens entscheidet, welches „Framework“ oder welche „grundlegenden Parameter“ für die Entstehung des eigentlichen Outputs verwendet werden. ${ }^{440}$ Zudem liege es in der

436 Dreier/Schulze-Schulze, UrhG, § 2 Rn. 8; Wandtke/Bullinger-Bullinger, PK UrhR, $\S 2$ Rn. 17; Schricker/Loewenheim-Loewenheim, UrhR, § 2 Rn. 41; Dreier, FS Kitagawa, S. 881.

437 Kummer, Das urheberrechtlich schützbare Werk; Kummer wollte für den Schutz „statistische Einmaligkeit“" genügen lassen.

438 DKM-Dreyer, HK-UrhG, § 2 Rn. 24; Lauber-Rönsberg, GRUR 2019, 244, 247.

439 Wandtke/Bullinger-Bullinger, PK UrhR, § 2 Rn. 17.

440 Dreier, FS Kitagawa, S. 881; für den aufmerksamen Leser dieser Arbeit klingt das schon sehr vielversprechend - „Auswahl des Frameworks“ und der (Hyper-) Parameter...; ähnlich inzwischen auch Loewenheim/Leistner, die als Voraussetzungen für einen Schutz fordern, dass der Mensch das ,wesentliche Grundmuster des Werks geschaffen hat und bei mehreren vom Computer erzeugten Versionen eine Auswahl vornimmt“", Schricker/Loewenheim-Loewenheim/Leistner, Urheberrecht, § 2 Rn. 41 m. w.N.. 
Natur der Sache, dass der Computer regelmäßig mehrere Varianten oder ganze Sammlungen produziert, und die kreative (bzw. menschlich-gestalterische) Handlung damit neben der Bestimmung des einzusetzenden Frameworks und der Parameter auch in der Auswahl des gewünschten Ergebnisses liege. ${ }^{441}$

d) Kombinierte Auswahltheorie

Ähnlich verfährt auch Gomille, der fordert, dass ein Urheberrechtsschutz auch bei einem Einsatz von Zufallsgeneratoren anerkannt wird, sofern der Künstler

„(1). das zugrundeliegende ästhetische Grundmuster selbst entwickelt hat, (2.) seine Anwendung Erzeugnisse von hinreichender individueller Gestaltungskraft hervorbringt und (3.) dieser Künstler über den Einsatz der Maschine sowie die Auswahl entscheidet, welche der maschinell generierten Erzeugnisse behalten und welche verworfen werden. “ 442

Dieser Vorschlag ist lediglich deshalb an dieser Stelle nicht ganz passend, weil das zweite Kriterium nach der hiesigen Vorgehensweise an späterer Stelle zu prüfen ist.

e) Externe Auswahltheorie

Noch einen anderen Ansatz vertritt Schulze, der die urheberrechtliche Schutzfähigkeit vorgefundener Werke dann zulässt, wenn Fachleute dem Gegenstand künstlerischen Wert beimessen, weil der Künstler durch die Präsentation des Gegenstands eine bestimmte Aussage trifft. ${ }^{443}$ Dieser Ansatz berücksichtigt jedoch nicht das Mitwirken des Urhebers an der Entstehung des Werkes und rückt damit in die Nähe der Kummer'schen Präsentationslehre. Damit erfasst diese Theorie die hier vorliegende Situation nicht ausreichend.

441 Dreier, FS Kitagawa, S. 881.

442 Gomille, JZ Nr. 20 2019, 969, 972.

443 Dreier/Schulze-Schulze, UrhG, § 2 Rn. 9. 


\section{f) Zusammenfassung}

Solange sich der Einsatz des Computers als Verwendung eines Hilfsmittels bzw. Werkzeugs darstellt, ist urheberrechtlicher Schutz unproblematisch möglich.

Der Schutz ist hingegen ausgeschlossen, wenn der gesamte Schaffensprozess vom Zufall gesteuert ist, und der Mensch an keiner Stelle eingreift.

Zwischen den Extremen spricht vieles für einen urheberrechtlichen Schutz, wenn der Künstler sich für den Einsatz eines Zufallsgenerators entscheidet, den Ergebnisraum durch die Auswahl der zur Verfügung stehenden „Bausteine“ und sonstige Eingabedaten an den Zufallsgenerator beschränkt (Schaffung des „Grundmusters“"444) und aus einer Vielzahl der generierten Ergebnisse eines auswählt, das seiner Vorstellung entspricht.

Denkbar wäre es zudem, eine menschlich-gestalterische Handlung auch dann anzunehmen, wenn die Begrenzung des Ergebnisraumes durch die Vorauswahl schon bewirkt, dass ausschließlich solche Ergebnisse entstehen, die der Künstler abstrakt erwartet, ohne dass eine Auswahl durch den Künstler erfolgt, sodass ihm dann alle entstehenden Ergebnisse zuzurechnen wären. ${ }^{445}$

Dagegen spricht jedoch zum einen, dass dies den Schutz zugunsten des Künstlers mitunter ausufern lassen könnte, wenn dadurch alle durch das eingesetzte Hilfsmittel erdenklichen Ergebnisse diesem einen Urheber zugerechnet würden. ${ }^{446}$

Zudem würde das an einen Stilschutz grenzen, der urheberrechtlich gerade nicht gewollt ist. ${ }^{447}$

Und nicht zuletzt scheint es denklogisch nicht konsequent, zum einen eine geistige Schöpfung bzw. eine gestalterische Handlung zu verlangen, zum anderen aber nicht zu fordern, dass der Urheber zumindest irgendwie auch das konkrete Ergebnis im Sinn gehabt haben könnte, was bei einer unendlichen Menge an Resultaten undenkbar ist. Der Urheber muss also zumindest durch irgend eine Art der Auswahl seine Verbindung zu dem geschaffenen Werk zum Ausdruck bringen, damit es ihm als Ergebnis seiner menschlich-gestalterischen Handlung zugerechnet werden kann.

444 Schricker/Loewenheim, Urheberrecht, $§ 2$ Rn. 41; Wandtke/Bullinger-Bullinger, PK UrhR, § 2 Rn. 16.

445 So im Ergebnis auch Wandtke/Bullinger-Bullinger, PK UrhR, § 2 Rn. 16: „Entscheidend (...) ist alleine, dass der von dem Menschen erbrachte Beitrag die gestalterische Schwelle des § 2 Abs. 2 überschreitet.“

446 So auch Gomille, JZ Nr. 20 2019, 969, 972.

447 Vgl. z. B. Dreier/Schulze-Schulze, UrhG, § 2 Rn. 46. 


\section{Vom Bekannten zum Unbekannten: KI-Kunst}

Weniger relevant war bis vor kurzem noch die Diskussion über „Kunst“, die von „künstlicher Intelligenz“ „,geschaffen“ wurde. Auch wenn es „KI-Kunst“ bereits mindestens seit den Sechzigern gibt, ${ }^{448}$ hat das Thema durch die rasante technische Entwicklung auf dem Gebiet des maschinellen Lernens in den letzten Jahren vermehrt Aufmerksamkeit erfahren. Als „KI-Kunst“ wird im Rahmen der folgenden Ausführungen ausschließlich urheberrechtlich relevanter Output (also Erzeugnisse, die sich den Werkkategorien des $\S 2$ Abs. 1 UrhG zuordnen oder sonst als Werk im Sinne von $\S 2$ Abs. 2 UrhG klassifizieren lassen) von Modellen maschinellen Lernens verstanden. In der Regel wird es sich dabei um Output handeln, der mithilfe generativer Systeme - insbesondere sogenannten Generative Adversarial Networks (GANs) ${ }^{449}$ erzeugt wird.

Die Ergebnisse von Klassifizierungen und Regressionsberechnungen zum Beispiel in Form von Wahrscheinlichkeitswerten und Labelzuweisungen sind damit explizit nicht gemeint, ebensowenig die optimierten Parameterwerte. Der Begriff der „KI-Kunst“ ist ferner nicht als auf Bild- und Musikwerke beschränkt zu verstehen, sondern meint auch literarische und sonstige Werke, solange diese Output eines ML-Modells sind.

Spätestens seit den Berichten ${ }^{450}$ über die Projekte „Next Rembrandt“‘451 und „Edmond de Belamy“452 ist die Diskussion über KI-Kunst in der (zunehmend auch rechtswissenschaftlichen) Öffentlichkeit angekommen. Unklar ist dennoch bislang eine tiefergehende Analyse dahingehend, wie auch im Rahmen solcher und anderer KI-Kunstwerke Urheberrechtsschutz entstehen kann. Springender Punkt scheint auch hier wieder die Zurechenbarkeit zu einem menschlichen Autor zu sein, die je nach Komplexität des eingesetzten Modells angezweifelt wird. ${ }^{453}$ Specht-Riemenschneider unterscheidet nach einer Vorbereitungs- und einer Gestaltungsphase, und stellt fest, dass es

448 Vgl. z. B. „AARON“ (1968) von Harold Cohen, beschrieben in Miller, The Artist in the Machine, S. 40.

449 Vgl. zur Erklärung von GANs § 2 B.II.3..

450 Vgl. z. B. Ahlberg/Götting-Stollwerck, BeckOK-UrhG, Europäisches Urheberrecht Rn. 119 f.; Linke/Petrlik, GRUR Int. 2020, 39, 43; Lauber-Rönsberg, GRUR 2019, 245 ff.; Ory/Sorge, NJW 2019, 710 ff., Dornis, GRUR 2019, 1252, 1253.

451 https://www.nextrembrandt.com/ (Stand: 22.02.2021).

$452 \mathrm{https}: / /$ obvious-art.com/portfolio/edmond-de-belamy/ (Stand: 22.02.2021).

453 Lauber-Rönsberg, GRUR 2019, 244, 248; Ory/Sorge, NJW 2019, 710,712; Dornis, GRUR 2019, 1252, 1256; Dreier, FS Kitagawa, 686, 879; Specht-Riemenschneider, FS Taeger, 711, 712 . 
für urheberrechtlichen Schutz auf ein Wirken des Menschen in der Gestaltungsphase ankomme, während ein Wirken in der Vorbereitungsphase nicht ausreiche. ${ }^{454}$ Die Gestaltungsphase sei dabei ,die Phase nach Anschaffung und Inbetriebnahme der Künstlichen Intelligenz", die Vorbereitungsphase hingegen die „Phase der Anschaffung und Inbetriebnahme“. ${ }^{455}$ Diese Trennung ist nach den in dieser Arbeit analysierten und dargelegten Grundlagen von ML-Systemen nicht immer treffend. Sie berücksichtigt insbesondere nicht, dass einige KI-Künstler die ML-Modelle selbst entwickeln, eine „Beschaffung und Inbetriebnahme“ erfolgt also nicht bzw. die Künstler treffen wesentliche Gestaltungsentscheidungen in deren Rahmen, sodass die Grenzen zwischen den vorgeschlagenen Phasen verschwimmen.

3. Wann liegt beim Einsatz von KI menschlich-gestalterisches Handeln vor?

Die Grundlagen für das Vorliegen eines Zurechnungszusammenhangs im Rahmen von Computerkunst wurden bereits erläutert. ${ }^{456} \mathrm{Zu}$ klären ist noch, ob, und wenn ja, warum KI-Kunst anders zu behandeln ist als traditionelle Computerkunst oder Computerkunst unter Einsatz von Zufallsgeneratoren.

\section{a) Problemstellung}

Zur Veranschaulichung sollen zum Einstieg zwei Extreme einer Skala betrachtet werden: Malt ein Maler ein Bild - mit Pinsel und Farbe auf die Leinwand - wird keiner den Zusammenhang zwischen menschlich-gestalterischer Tätigkeit und Endprodukt abstreiten. Dass mitunter Zufallselemente in das Ergebnis einfließen, weil der Pinsel abrutscht oder die Leinwand eine Unebenheit aufweist, wird dabei außer Acht gelassen. Am anderen Ende der Skala: ein (zugegeben bisher fiktives) autonomes System, das ohne das Zutun eines Menschen (also auch ohne die vorherige Programmierung, ,schöpferisch“ tätig zu werden $)^{457}$ die Initiative ergreift, und ein Ergebnis produziert - etwa ein Gemälde oder ein Musikstück. Oder der durch Medienberichte

454 Specht-Riemenschneider, FS Taeger, 711, 712.

455 Dies., FS Taeger, 711, 718.

456 Vgl. Abschnitt $\$ 9$ B.I.1..

457 Solche - nach hiesiger Ansicht zumindest bisher fiktiven - Konstellationen diskutiert für den Urheberrechtsschutz Legner, ZUM 2019, 807, 808 ff.. 
bekannte Fall des „Selfie-Affen“, in dem ein Affe zu einer Kamera griff und ein Selbstportrait anfertigte. ${ }^{458}$ In den beiden letzteren Fällen kann durch den fehlenden Einfluss eines Menschen ein schöpferischer Zusammenhang eindeutig abgelehnt werden. ${ }^{459}$

Sämtliche dazwischenliegenden Fälle erfordern scheinbar eine Einzelfallbetrachtung. Häufig helfen die menschliche Intuition und die Fähigkeit, Parallelen zu erkennen (so sind wir etwa in der Lage, einen Maler, der sich mit dem Pinsel betätigt, urheberrechtlich in etwa einem Künstler gleichzusetzen, der am Computer in einem Malprogramm ein Gemälde produziert): Selbst wenn das Malprogramm über die Malfunktion hinausgehende Bearbeitungsmöglichkeiten bereitstellt, wie etwa Filter oder vorgefertigte Elemente, sind wir bereit, das Endergebnis trotzdem noch dem Künstler zuzusprechen, weil er über die Auswahl der einzusetzenden Mittel entscheidet.

Aber diese menschliche Intuition scheitert, wenn wir den Prozess nicht mehr verstehen, der zum Endergebnis führt, wie am Beispiel künstlicher neuronaler Netze unschwer erkennbar. Dabei sind zwei Ebenen des Verstehens zu unterscheiden: Die der allgemeinen (Urheber-)Rechtswissenschaft, und die der fachkundigen Modellentwickler. Während in der Urheberrechtswissenschaft aufgrund fehlender Hintergrundinformationen und Erfahrung eine Einordnung dieser Technologie große Probleme bereitet, so kann es doch durchaus Modelle geben, bei denen die erfahrene Entwicklerin eindeutig in der Lage ist, zu beurteilen, ob das Modell das Ergebnis produziert, das intendiert war. Gleichfalls sind aber auch Situationen denkbar, in denen auch die Entwickler nicht mehr konkret nachvollziehen können, warum ihre Vorgaben zu einem bestimmten Ergebnis geführt haben. Und dann wird es spannend.

Möglicherweise haben die KI-Künstler eine Vorstellung davon, welche Werte (Hyperparameter) sie ändern müssen, um ihrem gewünschten Ergebnis näherzukommen, sei es aus Erfahrung oder aus dem Austausch mit anderen Entwicklern. Oder sie probieren ,,auf gut Glück“ unterschiedlichste Kombinationen so lange aus, bis das Ergebnis ihren Vorstellungen entspricht. Müssen die Entwickler - oder „KI-Künstler“ - zu jedem Zeitpunkt verstehen, wie ihre Ideen das Ergebnis beeinflussen? Müssen sie selber den Zusammenhang zwischen ihrer vermeintlich menschlich-gestalterischen Tätigkeit und dem Output des Systems nachvollziehen können? Wo verläuft die Grenze zum schlichten (nicht urheberrechtlich schutzfähigen) Entdecken? Wo die Grenze

458 Hoeren/Sieber/Holznagel-Ernst, Handbuch Multimedia-Recht, Teil 7.1 Rn. 4.

459 Ehinger/Grünberg, KuR 4 2019, 232, 233. 
zur Wissenschaft? Und - sollte eine Nachvollziehbarkeit gefordert werden ist überhaupt beweisbar, dass der Künstler den Prozess nicht verstanden hat?

\section{b) Andernorts am Beispiel von ANGELINA vorgeschlagene Herange- hensweise}

Für ein absolut autonom agierendes System wird in der Literatur beispielhaft das Projekt ANGELINA angeführt ${ }^{460}$ und beschrieben als eine Anwendung, die ,den Prozess der [Computer-]Spielentwicklung von Beginn an und bis zu dessen Beendigung beherrscht und dabei frei von gestaltender Einflussnahme arbeitet ". ${ }^{461}$ Dabei wird jedoch verkannt, dass die eigentliche Spielerzeugung gar nicht durch Anwendung maschineller Lernverfahren geschieht, sondern durch den Entwickler vorgegeben wird. ${ }^{462}$ Machine Learning kommt hier nur in Form sogenannter evolutionärer Algorithmen zum Einsatz, das heißt, die Software setzt ein Spiel bzw. Teile davon unter Verwendung der gesammelten Daten nach Vorgabe des Entwicklers zusammen, bestimmt dann unter Anwendung einer Fitness-Funktion die „Güte“ des Ergebnisses, und passt dann in der nächsten Iteration Parameter an. Ergebnis des Systems sind Textdateien (vergleichbar den Hyperparametern der hier erläuterten Machine Learning-Modelle, also ggf. dem Datenbankschutz zugänglich), die dann in einen anderen Softwareteil eingelesen werden können, der wiederum das Spiel ,produziert“. 463

Ehinger/Grünberg schlagen die folgende Vorgehensweise zur Abgrenzung zwischen dem Computerprogramm als Hilfsmittel und einem autonom schaffenden System vor: Zunächst sei zu klären, ob die Selektion, Kombination und Generierung der als Trainingsdaten eingesetzten Inhalte innerhalb der gewählten Datenbanken zufällig und unbeeinflusst von menschlichem Handeln erfolgen. ${ }^{464}$ Darüber hinaus müsse geprüft werden, ob die verwendete (Trainings-)Datenbank eine Größe erreiche, die das Erzeugnis in der Ge-

460 Ehinger/Grünberg, KuR 4 2019, 232, 235, verwiesen wird dort auf eine Präsentation auf YouTube (https://www.youtube.com/watch?v=UKzucHAZe3s (Stand: 22.02.2021), Informationen über das Projekt: http://www.gamesbyangelina.org (Stand: 22.02.2021); vgl. außerdem $\S 13$ B..

461 Ehinger/Grünberg, KuR 4 2019, 232, 235.

462 Cook, A Vision for Continuous Automated Game Design, S. 55.

463 Cook/Colton/Gow, IEEE Transactions On Computational Intelligence and AI in Games 9 Nr. 3 2017, 254, 256.

464 Ehinger/Grünberg, KuR 4 2019, 232, 235. 
samtschau ,vorher nicht hinreichend bestimmen“ lasse. ${ }^{465}$ Wenn diese zwei Fragen bejaht würden, sei demnach das Vorliegen eines reinen Hilfsmittels ausgeschlossen und damit auch eine persönliche geistige Schöpfung. ${ }^{466}$

Es lohnt sich jedoch ein genauerer Blick hinter die Kulissen des Systems um festzustellen, ob ANGELINA tatsächlich so autonom agiert wie dargestellt. Der Entwickler von ANGELINA beschreibt die Vorgehensweise bei der Entwicklung des ANGELINA-Systems wie folgt:

Zunächst werden passende (Software)-Bibliotheken ausgewählt, die für die Erzeugung des Spiels eingesetzt werden - diese ,beeinflussen den Designraum, den das System erkundet ${ }^{\star 467}$, der Mensch beschränkt also schon hier das Ergebnis.

Anschließend werden für die Konzepte bzw. Spielfeatures, die das System zur Entwicklung einsetzt, Abstraktionen vorgegeben ${ }^{468}$ - auch hier erfolgt eine maßgebliche Beschränkung.

Und auch in Bezug auf die ,autonome“ Auswahl von Internetquellen in späteren Versionen, die möglicherweise eine ,konkrete Determiniertheit der Ergebnisse ${ }^{\text {“469 }}$ verhindern könnte, werden maßgebliche Einschränkungen vorgenommen: Das System wählt den Input - z. B. in Form von Nachrichtenartikeln auf den Webseiten der Zeitschrift „The Guardian“- nicht eigenständig, sondern anhand einer zuvor durch die Entwickler vorgegebenen Priorisierung aus (unter anderem Datum des Artikels, Neuheit der beschreibenden Stichworte bzw. „Tags“, Erwähnung von Personen, die zuvor nicht erwähnt wurden) ${ }^{470}$ Diese Vorgehensweise ist vollständig determiniert, und könnte auch von einem Menschen durchgeführt werden.

Im Anschluss an diese vorbereitenden Handlungen kommt ein System evolutionärer Entwicklung zum Einsatz. Hierbei werden verschiedene Komponenten, die in der Vorbereitungsphase erzeugt wurden, automatisch miteinander kombiniert und durch „KI-Tester“ (automatisierte, ,intelligente“ Systeme, die anstelle von Menschen das Spiel testen) evaluiert. ${ }^{471}$ Das Ganze

465 Ehinger/Grünberg, KuR 4 2019, 232, 235.

466 Dies., KuR 4 2019, 232, 235.

467 Cook/Colton/Gow, IEEE Transactions On Computational Intelligence and AI in Games 9 Nr. 3 2017, 254, 256.

468 Cook/Colton/Gow, IEEE Transactions On Computational Intelligence and AI in Games 9 Nr. 3 2017, 254, 256.

469 Ehinger/Grünberg, KuR 4 2019, 232, 235.

470 Cook/Colton/Gow, IEEE Transactions On Computational Intelligence and AI in Games 9 Nr. 3 2017, 254, 257.

471 Cook/Colton/Gow, IEEE Transactions On Computational Intelligence and AI in Games 9 Nr. 2 2017, 192, 193 f.. 
stellt einen Optimierungsvorgang dar, bei dem sich der Mensch wiederum Zeit spart und das System als Hilfsmittel einsetzt.

Der Entwickler von ANGELINA muss (zumindest zum derzeitigen Stand) eingreifen, um einen Spielentwicklungsprozess zu beenden (vgl. o. a. Video bei ca. Minute 12:38, ,it ends when I tell it to“), er bestimmt also das Ergebnis. Und damit liegt wiederum eine menschlich-gestalterische Tätigkeit vor, weil der Mensch das Ende des Optimierungsprozesses vorgibt und damit erkenntlich macht, wann das Ergebnis seinen Vorstellungen entspricht. Darüber hinaus ist der Prozess, der in dem Video beschrieben wird (ANGELINA als fortlaufendes dauerndes System mit eigener Aufgabenpriorisierung etc.) bisher lediglich eine Vision. Ob die dadurch ggf. entstehende „Computational Creativity“ eines Tages an dieser Stelle zu anderen Subsumtionsergebnissen führen könnte, wird an anderer Stelle in dieser Arbeit diskutiert. ${ }^{472}$ Bisher ist ANGELINA demnach als Hilfsmittel einzuordnen.

\section{c) Kritik an dieser Vorgehensweise}

Für die zuvor dargestellte von Ehinger/Grünberg vorgeschlagene Vorgehensweise zur Abgrenzung ${ }^{473}$ zwischen menschlicher Schöpfung und nicht schutzfähiger Maschinenleistung bedeutet das: eben jenes Prüfungsschema gestaltet sich zu eng. Es berücksichtigt nicht, dass dem Entwickler - bzw. Urheber - bis dato stets auch noch eine auswählende und kontrollierende Rolle zukommt. Damit hält er die Fäden über das Endergebnis nach wie vor in der Hand. Auch eine unüberschaubar große Input-Datenbank kann zu vorhersehbaren Ergebnissen führen, wenn die Auswahlkriterien für den Inhalt der Datenbank konkret bestimmt sind.

\section{d) Lösungsvorschlag}

Ein Unterschied der KI-Kunst zur klassischen Computer- und Zufallskunst liegt darin, dass der Entwickler nicht (nur) bewusst Entscheidungen dem

472 Vgl. unten $\S 13$.

473 Vgl. b), Prüfung anhand von zwei Fragen: 1) Zufällige Auswahl der Trainingsdaten? 2) Lässt die Größe der Trainingsdatenbank das Erzeugnis in der Gesamtschau noch vorher hinreichend bestimmbar erscheinen? 
Zufall überlässt, sondern mit seinen Handlungen beabsichtigt, das Ergebnis unmittelbar zu beeinflussen. ${ }^{474}$

Ein anderer Unterschied wird darin gesehen, dass der Künstler das Grundmuster nicht konkret vorgibt, sondern durch die Maschine ermitteln lässt. ${ }^{475}$ Bei genauerer Betrachtung der Lage fällt auf, dass diese Unterschiede jedoch nicht dazu führen müssen, dass eine neue Bewertung erfolgt. Denn wenn der Urheber ,nur bis zu einem gewissen Grad“ das Ergebnis beeinflussen kann, so ist nicht zu verkennen, dass er das Ergebnis beeinflussen kann, und darin liegt ein Mehr zum Einsatz von Zufallsgeneratoren.

Damit ein ML-Modell überhaupt Ergebnisse hervorbringt, muss einiges an Vorleistung erbracht werden: Der Künstler muss sich unter anderem für ein Modell entscheiden, muss unter Umständen Hyperparameter bzw. Architektur festlegen und sich für Trainingsdaten entscheiden (oder ein Modell wählen, das auf einem passenden Datensatz trainiert wurde). Diese Entscheidungen sind alle in Bezug auf das gewünschte Ergebnis durch den Menschen zu treffen. Solange diese Entscheidungen nicht der Maschine (oder dem Zufall) überlassen werden (was bis dato aufgrund der ständigen Weiterentwicklung praktisch undenkbar ist: Die vorgegebene Menge an Auswahlmöglichkeiten wäre quasi immer beschränkt, sodass auch hier implizit eine Auswahl getroffen würde) können also nur maximal die Maßstäbe angelegt werden, die wir auch schon für die Computer- bzw. Zufallskunst anwenden:

Maßgeblich ist, dass der Mensch ein System wählt, Parameter einschränkt und ein Ergebnis als Werk aus mehreren auswählt. ${ }^{476}$ Diese Entscheidungen muss ein Urheber von KI-Kunst zwangsläufig treffen, eine menschlichgestalterische Tätigkeit liegt also bei dem Einsatz heute verfügbarer - generativer - KI-Systeme (fast) immer vor. ${ }^{477}$

Dornis kritisiert hingegen, dass eine Zurechnung eines durch KI geschaffenen Werkes zu einem Menschen anhand des Vorgebens von Rahmenbedingungen und Parametern zu einer Verwässerung des Urheberrechts führe. ${ }^{478}$ Selbst wenn sehr enge Rahmenbedingungen vorgegeben werden, fehle es an

474 Auch wenn der Künstler danach strebt, die gestaltenden Entscheidungen der Maschine zu überlassen, muss er doch bei dem Einsatz selbstlernender Systeme immer zielführende Beschränkungen vornehmen und damit den Gestaltungsspielraum begrenzen.

475 Gomille, JZ Nr. 20 2019, 969, 972.

476 Vgl. dazu die „strenge Auswahltheorie“ von Dreier oben c) sowie die Ausführungen in $\mathrm{f}$ ).

477 Diese Aussage muss freilich zu dem Zeitpunkt überdacht werden, an dem Maschinen, ohne irgendeinen menschlichen Input zu erhalten, Ergebnisse erzeugen.

478 Dornis, GRUR 2021, 784, 789. 
einem eigenschöpferischen menschlichen Beitrag. ${ }^{479}$ Diese These baut unter anderem darauf auf, dass insbesondere ML-Modelle einen ,höheren Grad an Emanzipation“ ${ }^{“ 480}$ aufwiesen, da sie ,einen Teil ihrer Fähigkeiten erst nach dem Abschluss von Programmierung und Datentraining “ entwickelten ${ }^{481}$ und „die spontan entstehende algorithmische Architektur“ sich von der Ausgangskonzeption unterscheide. ${ }^{482}$ An dieser Stelle wird zunächst auf die in den Kapiteln 6 und § 2 B.II.2. beschriebenen Grundlagen der Modelle verwiesen. Danach ist klar, dass die Modelle (insbesondere dasjenige, auf das Dornis sich später bezieht - das zur Erzeugung von Belamy eingesetzte Modell) nicht so selbständig sind wie angenommen. Vielmehr erfolgt im Rahmen des Trainings nur eine Werteoptimierung, deren optimaler Zustand durch vom Menschen gewählte Parameter vorgegeben wird, und wobei sich die menschliche Einflussnahme insbesondere auch entgegen der Annahme von Dornis $^{483}$ durch die Anpassung der Hyperparameter nicht nur auf die Modelle, sondern auch auf die Ergebnisse auswirkt. Es erscheint mithin übereilt, für den Einsatz von ML-Modellen im Schöpfungsprozess grundsätzlich von dem Fehlen einer eigenschöpferischen menschlichen Leistung auszugehen.

Von einer möglichen Zurechnung ausgenommen ist allerdings ein fertig trainiertes System, das auf Knopfdruck eines Dritten Output produziert, ohne dass jemand eine Auswahl des Ergebnisses trifft, und ohne dass eine Selektionsentscheidung anhand vorgegebener konkreter Kriterien in das System integriert wurde, ${ }^{484}$ wenn es nicht durch den Entwickler bedient wird. Dann liegen für den Handelnden die genannten Kriterien nicht vor, weil er die KI nicht gestaltet, keine Rahmenbedingungen vorgibt, sondern nur benutzt. ${ }^{485}$ Bei der Person des Entwicklers wiederum fehlt die Auswahlentscheidung. ${ }^{486}$

Übertragen in die Praxis könnte das so aussehen: Ein KI-Künstler entwickelt ein generatives Modell, das Grafiken erzeugt. Der Grafikerstellungsprozess wird im Museum durch einen Besucher per Knopfdruck gestartet.

479 Dornis, GRUR 2021, 784, 788.

480 Ders., GRUR 2021, 784, 785.

481 Ders., GRUR 2021, 784, 785.

482 Ders., GRUR 2021, 784, 785

483 Ders., GRUR 2021, 784, 789.

484 Beispiel für ein System, das auf „Knopfdruck“ produziert, könnte Google DeepDream sein. Allerdings arbeitet DeepDream mit Input des Benutzers - dieser lädt ein Bild in das System, das dann verändert wieder ausgegeben wird - und damit liegt nicht der „,reine Knopfdruckfall“ vor, auch wenn nur ein Ergebnis zurückgegeben wird.

485 Vgl. auch Nordemann/Fromm-A. Nordemann, UrhR, § 2 Rn. 25.

486 So auch Gomille, JZ Nr. 20 2019, 969, 972. 
Eine Vorauswahl der fertigen, anzuzeigenden Grafiken erfolgt nicht, sondern das Modell gibt alle erstellten Grafiken auf einem Bildschirm aus, solange der Besucher auf den Knopf drückt. Hier fehlt nach den oben (f)) genannten Kriterien die menschlich-gestalterische Handlung: Denn für den KI-Künstler fehlt in Bezug auf den konkreten Betrachtungsgegenstand (die erzeugte Grafik) die Auswahlhandlung, während der Besucher keinerlei Möglichkeit hatte, den Entstehungsprozess zu beeinflussen. Die bloße Betätigung des Knopfes, die der Maschine mehr oder weniger Zeit zur Ergebnisproduktion gibt, kann sich nicht gestalterisch auswirken. In dieser Situation wäre die entstehende Grafik mithin gemeinfrei.

Sollte eine menschlich-gestalterische Handlung vorliegen, ergeben sich unterschiedliche Bewertungen in Bezug auf die Urheberpersönlichkeit erst auf der Ebene der Urheberschaft. Zu berücksichtigen ist darüber hinaus, dass an dieser Stelle nur die Frage des Vorliegens menschlich-gestalterischer Tätigkeit zu klären war. Die weiteren Prüfungsschritte (geistiger Gehalt, Wahrnehmbarkeit, Individualität) sind natürlich - fallbezogen - auch noch zu bearbeiten.

\section{Geistiger Gehalt}

Neben der Frage nach dem Vorliegen menschlich-gestalterischen Handelns ist zu erwägen, ob die Forderung nach geistigem Gehalt im Kontext von KISystemen besonderen Augenmerks bedarf. Ein Werk hat „geistigen Gehalt“, wenn der menschliche Geist in ihm zum Ausdruck kommt, ${ }^{487}$ das Werk muss also einen ,,vom Urheber stammenden Gefühlsinhalt“ haben, ,der auf den Leser, Hörer oder Betrachter unterhaltend, belehrend, veranschaulichend, erbauend oder sonstwie anregend wirkt" ${ }^{488}$ Während es bisher darum ging, ob der Mensch einen hinreichenden Beitrag zum Werk geleistet hat, um ihn als Urheber erscheinen zu lassen, geht es jetzt konkret um den Inhalt des Werkes. Ein strenger ästhetischer Gehalt ist dabei nicht (mehr) zu fordern, ${ }^{489}$ vielmehr geht die Forderung in Richtung einer ,,sinnlich wahrnehmbaren eigenschöpferischen Formgestaltung“" ${ }^{490}$

487 Schricker/Loewenheim-Loewenheim/Leistner, Urheberrecht, § 2 Rn. 45.

488 Dreier/Schulze-Schulze, UrhG, § 2 Rn. 12.

489 Dreier/Schulze-Schulze, UrhG, § 2 Rn. 12.

490 BGH GRUR 1985, 1041, 1047 - Inkasso-Programm; Dreier/Schulze-Schulze, UrhG, $\S 2$ Rn. 12. 
Fraglich scheint jedoch, ob ein Werk denkbar ist, das durch eine menschlichgestalterische Handlung entstanden ist und keinen geistigen Gehalt besitzt. Möglicherweise impliziert die Forderung nach einer ,gestalterischen“ Handlung bereits, dass diese einen geistigen Gehalt mit sich bringt. Unabhängig davon, an welcher Stelle man hier die Trennlinie ziehen möchte, sind insbesondere in der Bild- und Tonschöpfung mit bzw. durch KI hinreichend Ergebnisse vorstellbar und existent, die den menschlichen Geist anregen, zu Interpretationen inspirieren und mitunter auch kommunikativen Gehalt aufweisen (vgl. nur Edmond de Belamy ${ }^{491}$ ). So beeinflusst der Entwickler beispielsweise durch die Wahl der KNN-Struktur und der Trainingsdaten, ob das Ergebnis etwa eine Strichzeichnung darstellt oder einem Aquarellgemälde ähnelt.

Die Anforderung des geistigen Gehalts ist jedenfalls fallbezogen zu analysieren und kann nicht grundsätzlich allen KI-Schöpfungen den Werkcharakter versagen.

\section{Wahrnehmbarkeit, Individualität}

Die Fragen nach dem Vorliegen der Wahrnehmbarkeit der Schöpfung sowie der Individualität sind fallbezogen nach den üblichen Maßstäben zu prüfen. Die Herstellung des Werkes an sich ist für die Beurteilung nicht relevant, weshalb sich - im Unterschied zu der Prüfung des Vorliegens menschlichgestalterischen Handelns - keine Unterschiede zur urheberrechtlichen Analyse traditioneller Werke ergeben. Deshalb wird hier nicht abstrakt ausführlicher darauf eingegangen.

\section{Zusammenfassung}

Ziel der vorangegangenen Ausführungen war es, die urheberrechtlichen Schutzvoraussetzungen für Werke im Sinne des UrhG dahingehend zu analysieren, ob sie auch auf die besonderen Umstände im Kontext von mittels bzw. mit Hilfe von KI bzw. ML-Modellen geschaffenen Erzeugnissen angewendet werden können. Ausgehend von der im Urheberrecht üblichen Vorgehensweise zur Bestimmung der Schutzfähigkeit eines Werkes wurde insbesondere der Faktor einer menschlich-gestalterischen Handlung untersucht. Dabei wurde

491 Beschreibung des Projektes in $§ 10$ C.III.. 
festgestellt, dass für die Betrachtung der Schutzfähigkeit von mithilfe von KI geschaffenen Werken gewisse Parallelen zur Computer- bzw. Zufallskunst gezogen werden können. Der Einsatz von KI schließt die Schutzfähigkeit nicht per se aus, sondern erfordert eine differenzierte Betrachtung, die in der überwiegenden Zahl der Fälle aufgrund des aktuellen Standes der Technik noch zu einem urheberrechtlichen Schutz führen kann. Dies wurde losgelöst von der Person des Urhebers untersucht, da in einem Schaffensprozess mitunter verschiedene Personen als Urheber in Betracht kommen. Darauf wird im Folgenden einzugehen sein. 


\section{$\S 10$ Wem gebührt ein möglicher Schutz? - Einführung eines Flussdiagramms zur Urheberermittlung}

Die Feststellung, dass nach heutigem Stand der Technik kaum ein Einsatz von KI möglich ist, der zum relevanten Ausschluss einer zugrundeliegenden menschlich-gestalterischen Tätigkeit führen könnte, wenn mit KI entsprechende Erzeugnisse produziert werden, ist eine wichtige Grundlage für den nächsten Schritt: Nun ist zu bestimmen, wem urheberrechtlicher Schutz zugesprochen wird. Zu klären ist also, wem die menschlich-gestalterische Handlung zuzurechnen ist.

Zum einen stellt sich diese Frage nur dann, wenn mehrere Personen als Urheber infrage kommen, zum anderen wird im Zweifelsfall eine Einzelfallentscheidung getroffen werden müssen. Dieser Abschnitt soll daher einen Kriterienkatalog anbieten, welcher für ebendiese Einzelfallentscheidungen unterstützend herangezogen werden und dabei helfen kann, einfache Fälle direkt zu klären. Die Frage nach der Urheberschaft stellt sich mit dem Einsatz von KI-Systemen insbesondere deshalb, weil immer mindestens eine Person das System entwickelt hat, dieses aber nicht zwangsläufig von der selben Person zur Werkerzeugung genutzt wird. Vermehrt werden unterschiedlichste generative Systeme verschiedener Urheber zu größeren, komplexeren Systemen miteinander verbunden. Ein Flussdiagramm soll dabei im Wege einer Formalisierung des Mitwirkungsprozesses helfen, den Urheber zu identifizieren. ${ }^{492}$

Nach der Darstellung einiger aktuell vertretener Ansichten zur Urheberschaft im Kontext von KI-Erzeugnissen wird das vorgeschlagene Diagramm zunächst abgebildet, es folgt eine Erläuterung der verwendeten Symbolik. Daran schließt sich die Darstellung der zugrundeliegenden Gedanken an.

\section{A. Forschungsstand: Wer ist warum Urheber?}

Zentrum der Fragestellung ist $§ 7$ UrhG. Gemäß $§ 7$ UrhG ist Urheber derjenige, der Schöpfer des Werkes ist. Schon rein wortlogisch ist damit Urheber

492 Die „Entwicklung von Regeln, um den oder die menschliche(n) Autor(en) zu ermitteln" schlagen auch vor Freialdenhoven et al., IntellectualProperty 2020, 28, 31. 
die Person, deren persönliche geistige Schöpfung in dem Werk verkörpert ist. Infrage kommt nur eine natürliche Person, ${ }^{493}$ die Geschäftsfähigkeit ist aufgrund der Realaktqualität des Schöpfungsvorgangs unerheblich. ${ }^{494}$ Grundsätzlich wird die Urheberschaft gem. § 10 Abs. 1 UrhG für denjenigen vermutet, der auf dem Werkstück als Urheber bezeichnet ist. Wenn auf dem Werkstück eine Bezeichnung fehlt, muss derjenige, der die Urheberschaft für sich beansprucht, diese beweisen. ${ }^{495}$ Anders als bei „klassisch“ geschaffenen Werkstücken, wie etwa einem signierten Gemälde, ist das häufig der Fall bei digital erstellten Werken, die höchstens in den Metadaten noch einen Rückschluss auf das Gerät zulassen, mit dessen Hilfe das Werkstück erzeugt wurde.

Neben der Beweisfrage ergeben sich für KI-Künstler allerdings noch andere vorgelagerte Fragestellungen: Können sie überhaupt davon ausgehen, Urheber zu sein, wenn sie Machine Learning-Modelle einsetzen? Und welche Voraussetzungen müssen vorliegen, um diese Frage bejahen zu können?

Um in der Entwicklerrolle als Urheber zu gelten, wird unter anderem verlangt, dass derjenige bereits im Entwicklungsprozess die ,das Werk prägenden Parameter persönlich gestaltet" ${ }^{496}$ Dies wird nicht angenommen, wenn das Ergebnis für den Programmierer trotz der vorgegebenen und implementierten Regeln aus ex-ante-Sicht nicht vorhersehbar war. ${ }^{497}$ Es komme „maßgeblich darauf an, welche Auswirkungen die Gestaltungsentscheidungen des Programmierers auf das Endprodukt haben““ ${ }^{498}$ Dafür sei es insbesondere von Bedeutung, dass die Gestaltungsentscheidungen des Entwicklers nicht von Gestaltungsentscheidungen Dritter oder durch automatisierte Gestaltungsentscheidungen überlagert werden. ${ }^{499}$ Dies offenbart bereits eine wichtige Erkenntnis: Für Systeme, die mehrere Akteure einbeziehen (können), reicht es nicht aus, nur die Handlungen eines einzelnen Akteurs zu betrachten. Vielmehr muss eine Gesamtwürdigung erfolgen.

493 Dreier/Schulze-Schulze, UrhG, § 7 Rn. 2.

494 Dreier/Schulze-Schulze, UrhG, § 7 Rn. 3.

495 BGH GRUR 1986, 887, 888 - Bora Bora; Dreier/Schulze-Schulze, UrhG, § 7 Rn. 10.

496 Ehinger/Grünberg, KuR 4 2019, 232, 234.

497 Dies., KuR 4 2019, 232, 234.

498 Lauber-Rönsberg, GRUR 2019, 244, 248.

499 Dies., GRUR 2019, 244, 248. 


\section{B. Vorgeschlagene Vorgehensweise zur Ermittlung des Urhebers}

Das Flussdiagramm in Abbildung 10.1 verwendet Rauten zur Darstellung von Entscheidungen. Für einen gegebenen Fall erfolgt der Einstieg in das Diagramm oben links, und endet bei einer von vier Varianten:

1) Es ist nur eine Person beteiligt - nur diese Person kommt als Urheber in Betracht.

2) Urheber ist der Entwickler.

3) Urheber ist der Benutzer bzw. Verwender.

4) Es gibt keinen (menschlichen) Urheber.

Genau genommen dürften Systeme, die zu Ergebnis 4) gelangen, gar nicht auf Urheberschaft überprüft werden müssen, denn dort fehlt in der Regel schon die menschlich-gestalterische Handlung.

Wie in diesen Konstellationen dennoch über eine Urheberschaft oder alternative Konzepte nachgedacht werden könnte, wird in $\S 12$ C.II. diskutiert. Darum wurde dieser Zustand ebenfalls aufgenommen. Der Prüfende folgt also den Pfeilen entlang einer Reihe von Ja/Nein-Entscheidungen bis zum Ziel.

1. Hat dieselbe Person das System und alle Komponenten entwickelt, ggf. Input gegeben und ggf. ein Ergebnis ausgewählt? An dieser Stelle geht der weiteren Prüfung die Frage voraus, ob überhaupt mehrere Menschen als Urheber in Betracht kommen. Ansonsten erübrigt sich die weitere Prüfung. Wenn nur ein Urheber in Betracht kommt, ist zu untersuchen, ob das Erzeugnis tatsächlich die Idee dieses Urhebers verkörpert. Ausreichend ist nicht, dass der Entwickler die Rechte an allen Bestandteilen des Systems hat. Vielmehr muss er Urheber des Systems im Ganzen sein, also zum Beispiel auch die einzelnen Module programmiert und ausgewählt haben. Es reicht also nicht aus, wenn die Rechte an dem System von einem anderen Urheber erworben wurden (dann kommen letztlich auch wieder mindestens zwei verschiedene Personen in Betracht). System meint die Software mit allen Bestandteilen, die für die Erzeugung des Outputs eingesetzt wird, inklusive der Machine-Learning-Komponenten. Input meint Informationen (nicht nur, aber auch Grafiken und Texte), die dem System nach der Fertigstellung mit oder nach dem Start des Programms zur Verfügung gestellt werden. 


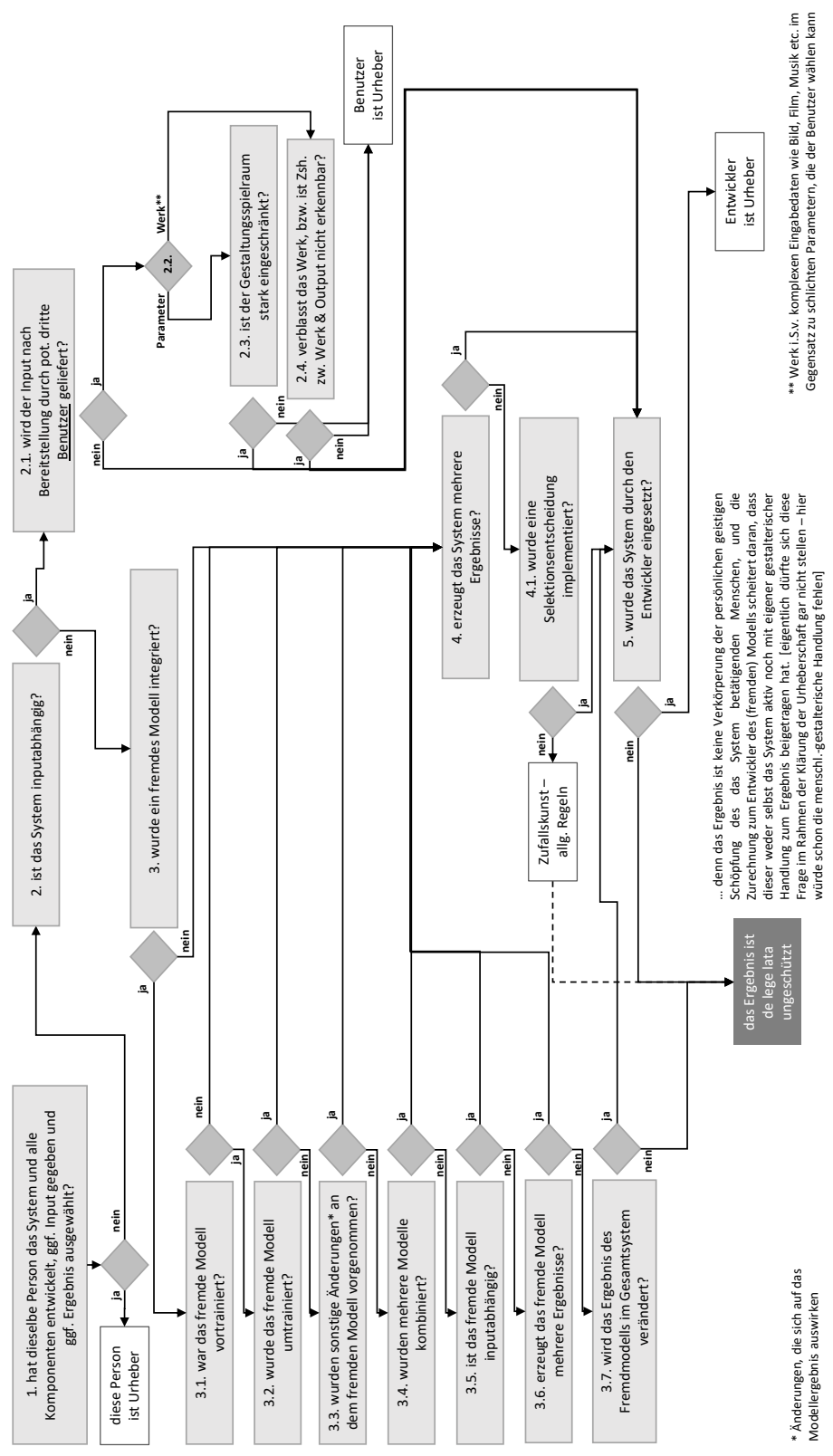

Abbildung 10.1: Flussdiagramm Urheberschaft. Quelle: eigene Darstellung. 
2. Ist das System inputabhängig? Ein starkes Indiz für Urheberschaft ergibt sich daraus, dass die Person, die das System zur Erzeugung von künstlerischem Output verwendet, das Ergebnis durch das Beisteuern eigener Daten (von reinen Parametern bis hin zu Bildern oder Texten) beeinflusst. Das System ist inputabhängig in diesem Sinne, wenn es so gestaltet ist, dass es ab dem Zeitpunkt des Ablaufenlassens den Benutzer um Input bittet, und dieser Input sich auf den Output derart auswirkt, dass der Benutzer den Output dadurch in gewisser Weise - wenn auch indirekt - steuern kann. Das System ist also nicht inputabhängig, wenn der gegebene Input für das Ergebnis im wesentlichen unbeachtlich ist, etwa weil sich dadurch nur minimalste Änderungen ergeben. Zweifeln bzgl. der Inputabhängigkeit könnte mithilfe des Einsatzes von Methoden zur Erklärung von KI-Systemen begegnet werden. Mithilfe dieser Methoden (auch unter dem Stichwort „Explainable AI“ bzw. „XAI“ bekannt) kann beispielsweise bildlich dargestellt werden, welche Teile des Inputs zu welchen Teilen des Outputs geführt haben. ${ }^{500}$

\subsection{Wird der Input nach Bereitstellung durch potenzielle Benutzer geliefert?}

Diese Frage bezieht sich darauf, ob Dritte das System zur Outputerzeugung einsetzen. „Bereitstellung“ meint, dass das System einsatzbereit zur Verfügung gestellt wird. Wenn diese Frage mit ,ja “ beantwortet wird, hat ein Dritter - oder der Entwickler in der Rolle eines Verwenders - nach Fertigstellung des Systems diesem persönlich gewählte Vorgaben zur Erzeugung des Ergebnisses gemacht und damit Einfluss auf die Outputerzeugung genommen. Für den Fall, dass eine Schutzfähigkeit gegeben ist (diese ist - an dieser Stelle sei das nochmals zu betonen - gesondert festzustellen) kommt damit nur der Benutzer als Urheber in Betracht. Die Urheberschaft des Entwicklers scheidet dann aus, weil er das System nicht eingesetzt und den beeinflussenden Input nicht gewählt hat. Wenn Frage 2.1. mit „,nein “ beantwortet wird, kann es sich immer noch um ein inputabhängiges System handeln, das allerdings den Input bereits vor oder mit Bereitstellung zugeführt bekommt. Ein solches System könnte so aussehen, dass der Entwickler ein Bild wählt - etwa „Sternennacht“ von van Gogh - und den Zugriff auf das Bild im System fest verankert. Wenn es sich beispielsweise um ein System handelt, das bestimmte Elemente in ein bestehendes Werk integriert, oder besondere Objekte hervorhebt, dann ist dieses System inputabhängig, aber ein (Dritter) Benutzer, der das System einsetzt, kann keinen Einfluss mehr auf das Ergebnis nehmen, weil er den Input nicht auswechseln kann. Für

500 Vgl. ausführlicher dazu Käde/von Maltzan, CR 2020, 66 ff.. 
den Dritten kommt daher mangels Einflussmöglichkeit eine Urheberschaft in der Regel nicht in Betracht. Für den Entwickler kommt hingegen eine Urheberschaft nur dann infrage, wenn er das System selber einsetzt. Andernfalls fehlt - auch wieder im Rahmen der Schutzfähigkeit zu klären - die Wahrnehmbarkeit des Werkes, es handelt sich allenfalls um eine Vorstellung von dem Werk, die noch nicht konkretisiert wurde.

2.2. Art des Inputs Neben der Bereitstellung von ,echten“ Inputdaten wie Bildern, Texten, Filmen, Musikstücken etc. (hier Werk genannt) ist auch vorstellbar, dass das System den Benutzer Parameter wählen lässt. Je nach Art des Inputs stellen sich weitere Folgefragen. Parameter sind hier nicht Variablen in ML-Modellen, sondern vom Benutzer justierbare Einstellungen, die sich auf das Endergebnis auswirken. Für einen Bildkunstgenerator könnte das etwa eine Epoche bzw. ein Stil sein, Farben, ein abbildungsbeschreibender Text oder Ähnliches, für einen Musikgenerator z. B. die Tonart, Tempo, Musikstil, Tonraumbegrenzung, Taktart und dergleichen.

2.3. Ist der Gestaltungsspielraum stark eingeschränkt? Für den Fall, dass der Benutzer keine Daten bereitstellt, sondern Parameter wählt - also quasi Einstellungen am System vornimmt - ist zu fragen, ob die Auswahlmöglichkeiten derart begrenzt sind, dass nicht davon gesprochen werden kann, dass das Erzeugnis die Verkörperung einer Idee des Benutzers darstellt, weil der Gestaltungsspielraum nicht ausreichend groß ist. In einem solchen Fall kommt - auch wenn der Schutz zugunsten des Nutzers daran scheitert, dass der Benutzer dem Ergebnis nicht genügend eigene Prägung verliehen hat, sodass über einen Schutz zugunsten des Entwicklers nachgedacht werden könnte - auch der Entwickler nicht als Urheber in Betracht, weil er das System nicht selbst eingesetzt hat. ${ }^{501}$ Damit ist in einem solchen Fall das Erzeugnis als gemeinfrei einzuordnen. Daraus ergeben sich aber insbesondere für den Benutzer regelmäßig keine Probleme, weil er das Erzeugnis zu allen beabsichtigten Zwecken einsetzen kann - er kann dies nur nicht exklusiv tun, das heißt, er kann andere nicht von urheberrechtlich geschützten Handlungen ausschließen.

2.4. Verblasst das Werk, bzw. ist der Zusammenhang zwischen Werk und Output nicht erkennbar? Wenn der Benutzer nicht lediglich Parameter justiert, sondern dem System komplexe Inputdaten - also ein (nicht notwendiger-

501 Vgl. zur Begründung d). 
weise urheberrechtlich geschütztes) Werk - zugeführt hat, ist zu fragen, ob der Benutzer durch die Auswahl des Inputs den erzeugten Output so weit steuern kann, dass das originäre Werk hinter dem Ergebnis dieses Eingriffs verblasst. Wenn sich das Ergebnis trotz komplexer Inputdaten nicht als von dem Benutzer intendiertes Erzeugnis darstellt, verschiebt sich die Einstufung des Systems von der Eigenart als (steuerbares) Hilfsmittel zunehmend in Richtung ,Zufallsgenerator“. Dann wäre mit dem oben unter d) Gesagten zu fordern, dass der Benutzer ein System wählt (das wird regelmäßig implizit gegeben sein), Parameter einschränkt (hier ist dann maßgeblich, ob der Input parametereinschränkende Wirkung hat) und ein Ergebnis als Werk aus mehreren auswählt (inputbasierte Systeme erzeugen häufig nur ein Ergebnis, sodass die Auswahl aus mehreren Ergebnissen sich dann daraus ergeben könnte, ob der Benutzer das System beispielsweise iterativ einsetzt, indem er immer wieder den Output als Input zuführt, um dann zu bestimmen, wann das Ergebnis seinen Vorstellungen entspricht. Das setzt allerdings technisch voraus, dass der Output und der Input ähnlicher Natur sind, etwa Bild und Bild wie bei Google DeepDream).

Fraglich ist, wann der Input als hinreichend ,parametereinschränkend“ zu bewerten ist. Hinter der Forderung nach einer Parametereinschränkung steht die Absicht, dem Benutzer zumindest eine gewisse Steuerungsintention in Bezug auf das Endergebnis abzuverlangen. Er soll das System nicht ,wie vorgefunden " übernehmen, sondern durch die Auswahl von Parametern einen Einfluss auf das Ergebnis nehmen. Wenn das System den Input lediglich verändert als Output wieder ausgibt (wie im Fall von Google DeepDream) ist der Steuerungseinfluss aufgrund der großen Abhängigkeit des Outputs vom Input in der Regel gegeben.

Wenn der Input in Form eines Filmes vorliegt, für den das System einen Soundtrack erzeugt, kann die Steuerungsmöglichkeit durch die Auswahl der Filmsequenzen als Input gegeben sein: es spricht Vieles dafür, dass der Benutzer dadurch, wie er den Film gestaltet, der Musik eine Richtung vorgibt. Möglich ist aber auch, dass hier kein hinreichender Zusammenhang zu sehen ist. Dann wäre die Musik gemeinfrei (mangels Handlung des Entwicklers des Systems) und der Benutzer könnte sie - wie auch schon in 2.3. - bedenkenlos einsetzen. Oder sie wäre dem Entwickler zuzuordnen, wenn er hinreichend Selektionsentscheidungen implementiert hat. Hierfür hat eine fallbezogene Betrachtung zu erfolgen.

3. Wurde ein fremdes Modell integriert? Falls sich der Systementwickler bzw. KI-Künstler im Falle eines nicht-inputbasierten Systems eines Fremdmodells 
bedient und dieses in sein System integriert hat, ist zu klären, ob noch ausreichend Eigenleistung an dem erzeugten Ergebnis erkennbar ist, um dem Entwickler des Systems die Urheberschaft zuzusprechen. Gleich vorweg: Eine Urheberschaft des Entwicklers eines fremden Machine-Learning-Modells am Erzeugnis kommt nicht in Betracht. Selbst wenn sich das Ergebnis ausschließlich der Leistung des fremden Modells zurechnen ließe, fehlt die entscheidende Handlung durch den Entwickler des Fremdmodells, der die Werkerzeugung hier nicht veranlasst. Im Übrigen helfen auch die Ansätze, die den Ergebnissen von Computerprogrammen als Ausdruck derselben Schutz zusprechen, nicht weiter, denn reine ML-Modelle (ohne den assoziierten Code) sind - wie gezeigt ${ }^{502}$ - dem Computerprogrammschutz nicht zugänglich.

Für den Fall der Integration eines Fremdmodells liegt auch die Frage nach einer eventuellen Miturheberschaft gem. § 8 UrhG auf der Hand. Die Voraussetzung dafür ist, dass mehrere gemeinsam ein Werk schaffen, ohne dass sich ihre Anteile gesondert verwerten lassen (vgl. § 8 Abs. 1 UrhG). Es geht hier nach wie vor um das Werk als Ergebnis des ML-Prozesses, nicht um das ML-System, sodass auch die genannten Anteile der Schaffenden in Bezug auf das Werk (und nicht etwa die beigesteuerten ML-Modelle) zu betrachten sind. Aus dem entstehenden „Werk“ - etwa einer Grafik - wird in der Regel nicht mehr zu erkennen sein, welcher Entwickler welchen Anteil beigesteuert hat. Erzeugt das System einen Film mit Soundtrack, und der eine Entwickler liefert ein Modell, das Filme generiert, während der andere ein Modell beisteuert, welches Musik zum Film erzeugt, kann schon eher über eine separierbare Verwertung nachgedacht werden. Dann ist eine spezifische Einzelfallbetrachtung vorzunehmen. Für weniger komplexe, in der Werkart homogene Erzeugnisse wird eine gesonderte Verwertung jedoch regelmäßig nicht in Betracht kommen. Dann ist eine Miturheberschaft denkbar.

Voraussetzung hierfür ist zunächst eine einheitliche Schöpfung. ${ }^{503}$ Diese liegt vor, wenn die Beteiligten willentlich zusammenwirken und sie sich einer einheitlichen Gesamtidee unterordnen - kennzeichnend sind insbesondere ein gemeinsamer Plan, ein gemeinsamer Wille und ein gemeinsames Ziel. ${ }^{504}$ Wenngleich nicht ausgeschlossen ist, dass mehrere Entwickler sich zusammentun, um ein besonderes System für die Erzeugung von Werken zu implementieren, so kann jedoch nicht davon ausgegangen werden, dass Entwickler,

$502 \S 7$ B.III..

503 Dreier/Schulze-Schulze, UrhG, § 8 Rn. 2.

504 Dreier/Schulze-Schulze, UrhG, § 8 Rn. 2. 
die unabhängig voneinander ML-Modelle konzipieren, dies im Hinblick auf die Fortentwicklung durch andere tun. Wenn also ein KI-Künstler ein fremdes ML-Modell auswählt und integriert, weil es ihm für seinen Zweck dienlich scheint, ohne dass dieses fremde Modell im Hinblick auf die Verwendung in seinem System entwickelt wurde, kann nicht von einer Miturheberschaft ausgegangen werden, schon weil die geistige und kommunikative Verständigung der Beteiligten fehlt.

Die folgenden Fragen zielen darauf ab, zu klären, ob das Fremdmodell verändert oder derart mit anderen Systemkomponenten verbunden wurde, dass sich das Erzeugnis dennoch dem Entwickler des Gesamtsystems zuschreiben lässt.

3.1. War das fremde Modell vortrainiert? Vortrainiert ist ein Modell, wenn es bereits mit auf einen ausgewählten Trainingsdatensatz optimierten Parametern vorliegt. Ein untrainiertes Modell ist im Wesentlichen eine Ansammlung von Hyperparametern und mehr oder weniger willkürlich gewählten Parametern und kann erst produktiv eingesetzt werden, wenn ein Training durchgeführt und die Parameter entsprechend optimiert wurden. Durch das Training wird das Modell in die Lage versetzt, die gewünschten Aufgaben durchzuführen. Wenn das fremde Modell nicht vortrainiert war, muss der Entwickler des Gesamtsystems das Modell folglich noch selbst konkretisieren. Dann kann von einem ausreichenden Beitrag des Entwicklers ausgegangen werden.

3.2. Wurde das fremde Modell umtrainiert bzw. erweitert? Falls ein vortrainiertes fremdes Modell eingesetzt wird, kann der Entwickler des Gesamtsystems entscheiden, das Fremdmodell auf einen anderen Datensatz zu trainieren (falls das Modell etwa bisher Stühle erkennen konnte, wäre es denkbar, das Modell auf Betten umzutrainieren bzw. zu erweitern). Möglicherweise reicht es dafür nicht einmal aus, dem Modell andere Trainingsdaten zu präsentieren, sondern ggf. sind darüber hinaus auch strukturelle Anpassungen vorzunehmen. In jedem Fall muss die Bejahung der Frage zurück in Richtung Urheberschaft des Entwicklers führen.

3.3. Wurden sonstige Änderungen an dem fremden Modell vorgenommen? An dieser Stelle sind alle anderen Veränderungen des Fremdmodells zu berücksichtigen, sofern sie sich nicht nur unwesentlich auf das Ergebnis des Gesamtsystems auswirken. 
3.4. Wurden mehrere Modelle kombiniert? Falls das Fremdmodell nicht verändert wurde, aber das Erzeugnis des Gesamtsystems sich als eine Kombinationsleistung aus verschiedenen Modellen darstellt, kommt auch hier eine Urheberschaft des Entwicklers des Gesamtsystems in Betracht, der die verschiedenen Modelle miteinander kombiniert hat. Diese Frage kann auch schon zu Beginn geklärt werden, sie wird an dieser Stelle nur der Vollständigkeit halber aufgenommen.

3.5. Ist das fremde Modell inputabhängig? Wenn das Fremdmodell inputabhängig ist (zur Definition der Inputabhängigkeit vgl. 2.) besteht auch an dieser Stelle die Möglichkeit des Entwicklers, das Ergebnis zu beeinflussen. Beispielsweise kann im Rahmen des Gesamtsystems ein Input abgerufen oder erzeugt werden, der dem Fremdmodell zugeführt wird, welches dann das Gesamtergebnis erzeugt.

3.6. Erzeugt das fremde Modell mehrere Ergebnisse? Für den Fall, dass das fremde Modell mehrere Ergebnisse erzeugt, könnte der Entwickler durch eine Auswahlhandlung seiner geistigen Schöpfung Ausdruck verleihen. Die Auswahl eines Ergebnisses aus den Fremdmodellerzeugnissen - z. B. auch durch eine implementierte Selektionsentscheidung, die nach vom Entwickler definierten Qualitätskriterien entscheidet - ist zwar keine für die Urheberschaft hinreichende Bedingung, jedoch verblasst dann der exklusive Einfluss des Fremdmodells auf das Gesamtergebnis. Damit muss die Prüfung wieder zurück in Richtung Urheberschaft des Entwicklers führen.

3.7. Wird das Ergebnis des Fremdmodells im Gesamtsystem verändert? Wenn das Fremdmodell auch nur ein Ergebnis erzeugt, kann der Entwickler des Gesamtsystems dennoch zur Urheberschaft gelangen, wenn sein entwickeltes Gesamtsystem das Erzeugnis des Fremdmodells nicht nur unwesentlich verändert. Wenn auch diese Frage mit einem „Nein “ beantwortet wird, kann in der Regel kein Urheber gefunden werden. Das Ergebnis bleibt daher - de lege lata - ungeschützt.

4. Erzeugt das System mehrere Ergebnisse? Diese Frage will dem Umstand Rechnung tragen, dass - wie im Kapitel zur Computerkunst auch für den Einsatz von Zufallsgeneratoren gefordert ${ }^{505}$ - die Auswahl eines Systems nicht ausreicht, um Schutz an einem damit erzeugten Ergebnis zu erlangen.

505 Vgl. § 9 B.I.1.. 
Vielmehr muss der potenzielle Urheber seine geistige Verbindung zu dem Ergebnis zum Ausdruck bringen. Dies kann er (in dem hiesigen Setting) tun, indem er in einem System, das mehrere Ergebnisse produziert, dasjenige auswählt, das seinen Vorstellungen entspricht.

\subsection{Wurde eine Selektionsentscheidung implementiert? Wenn das System} nicht mehrere Erzeugnisse ausgibt, kann es sein, dass das vom Entwickler so gewollt ist. Möglicherweise wurde im Gesamtsystem eine Vorauswahl implementiert, sodass der Entwickler die Entscheidung quasi vorgezogen und automatisiert vorgenommen hat (es wäre etwa denkbar, dass eine Selektion anhand einer bestimmten Pixel- oder Farbverteilung in einem Bild erfolgt). Wenn diese implementierte Selektionsentscheidung einer manuellen Auswahl gleichsetzbar ist, kann auch an dieser Stelle ein Urheber gefunden werden. Andernfalls fehlt auch hier die geistige Verbindung zwischen Ergebnis und Entwickler, das Erzeugnis stellt sich in dem Fall als ein $\mathrm{Zu}-$ fallsprodukt dar, das dem Entwickler nicht zuzurechnen ist.

5. Wurde das System durch den Entwickler eingesetzt? Diese Frage versucht, den finalen Bezug zwischen Entwickler und Ergebnis herzustellen. Auch wenn der Entwickler wesentliche prägende Schritte im Rahmen der Systemimplementierung vorgenommen hat, so kann ihm das Ergebnis als Werk doch nur dann zugerechnet werden, wenn er es auch ist, der das System zur Werkerzeugung einsetzt. Ansonsten steht auch in diesem Fall das Werk mangels Urheber schutzlos da.

Denkbar sind auch Konstellationen, in denen der Entwickler und der Benutzer auf eine Weise zusammenwirken, die eine Miturheberschaft beider infrage kommen lassen. Miturheberschaft setzt gem. § 8 UrhG grundsätzlich voraus, dass mehrere ein Werk gemeinsam schaffen, ohne dass sich ihre Beiträge gesondert verwerten lassen. Das gemeinschaftliche Schaffen setzt dabei voraus, dass beide Urheber bei der Umsetzung einer gemeinsamen Idee gewollt zusammenwirken. ${ }^{506}$ Vonnöten sind also ein gemeinschaftlicher Plan, ein gemeinsamer Wille und ein gemeinsames Ziel der Mitwirkenden. ${ }^{507}$ In den im Rahmen des Flussdiagramms berücksichtigten sowie den unten dargestellten Anwendungsfällen dürfte es in der Regel insbesondere an einem gemeinschaftlichen Plan fehlen: Zum einen erfolgt nur selten eine Kommunikation zwischen Benutzer und Entwickler, zum anderen dürfte

506 Dreier/Schulze-Schulze, UrhG, § 8 Rn. 2.

507 Dreier/Schulze-Schulze, UrhG, § 8 Rn. 2. 
es insbesondere im Kreativgewerbe regelmäßig zu vom Entwickler nicht beabsichtigen Einsätzen seiner Anwendung kommen, um neue und unerwartete Werke zu produzieren. Wenn doch einmal ein Entwickler für einen bestimmten Benutzer ein Modell entwickelt, und dieser im Anschluss an die Entwicklung unter Berücksichtigung des gemeinsamen Ziels der Werkerstellung dieselbe initiiert, könnte ggf. eine Miturheberschaft beider Beteiligten anzunehmen sein. Dies ist jedoch im Rahmen einer Einzelfalluntersuchung gesondert festzustellen. Gleiches gilt für die arbeitsteilige Entwicklung eines ML-Modells, wenn die Schutzentscheidung zugunsten des Entwicklers ausfällt: Auch dann kommen mehrere Entwickler als Miturheber infrage.

\section{Verwendung des Flussdiagramms anhand von Beispielen}

Zur Demonstration der Arbeitsweise mit dem vorgestellten Flussdiagramm folgen nun einige beispielhafte Anwendungen. Dabei ist jeweils zu klären, wer Urheber des KI-Erzeugnisses ist. Untersucht werden nur solche Systeme, die Machine Learning-Komponenten zur Ergebniserzeugung einsetzen, also in der Regel generative ML-Modelle.

\section{Google DeepDream}

DeepDream ist der Name für eine Implementierung des Inception-MLModells von Google, die eine Bilddatei als Eingabe erfordert, dann das eingegebene Bild analysiert und überarbeitet wieder ausgibt. Das zugrundeliegende ML-Modell findet Strukturen in dem hochgeladenen Bild, und hebt diese im Ergebnis besonders hervor. ${ }^{508}$ Das Modell erkennt dabei nur solche Strukturen, die es vorher in Form von Trainingsdaten ,kennengelernt" hat. Die Arbeitsweise von DeepDream hängt maßgeblich davon ab, mit welchen Einstellungen das Netzwerk bereitgestellt wird - DeepDream ist eine Anwendung, die nicht von Google selbst funktionsfähig abrufbar ist, vielmehr steht der Quellcode auf GitHub ${ }^{509}$ zur freien Verfügung (veröffentlicht unter der Apache 2.0-Lizenz). Eine Websuche liefert einige Anbieter,

508 https://ai.googleblog.com/2015/06/inceptionism-going-deeper-into-neural.html (Stand: 22.02.2021).

509 https://github.com/google/deepdream (Stand: 22.02.2021). 

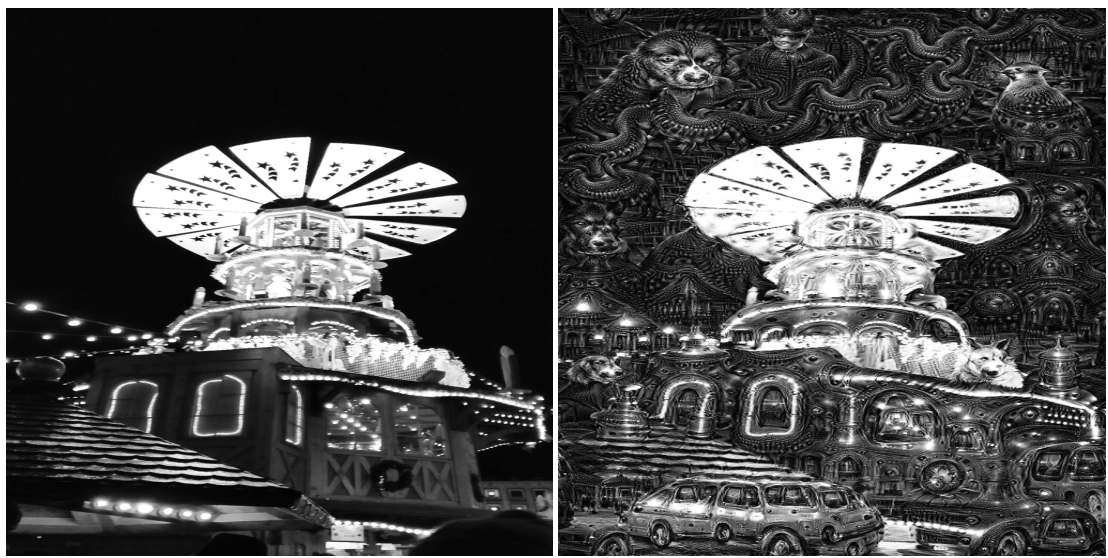

Abbildung 10.2: DeepDream - Links: Input, Rechts: Output (Quelle: eigene Darstellung).

die DeepDream als Webanwendung zur Verfügung stellen. ${ }^{510}$ Dort kann ein Benutzer ein eigenes Bild hochladen, und bekommt wenig später den Output von DeepDream zurück.

Die Abbildung 10.2 stellt Input und Output gegenüber. Dadurch wird besonders eindrucksvoll die Abhängigkeit von dem vorgegebenen Input deutlich. Zur Erzeugung dieses Outputs in einer derart ausgeprägten Form war es erforderlich, das Original in der von DeepDream zurückgegebenen Version mehrfach iterativ dem System erneut zuzuführen. Zudem brachte erst eine nachträgliche Aufhellung des Bildes die Strukturen vollständig zum Vorschein.

Die Anwendung des vorgestellten Flussdiagramms führt damit recht schnell zu Punkt 2.1. des Schemas, mit dem Ergebnis, dass die Urheberschaft hier beim Benutzer zu verorten ist. Der gewählte Anbieter des Systems gibt dem Benutzer keine Möglichkeit, Einstellungen an dem Modell vorzunehmen. Solche Einstellungen (Parameter) könnten beeinflussen, wie intensiv das Modell bestimmte Formen im Bild erkennt, wodurch der Benutzer das Ergebnis nach seinen Vorstellungen optimieren (und der Input mithin deutlich stärker oder weniger stark verfremdet) werden könnte. Das Ergebnis könnte sich dann unter Umständen deutlich stärker vom eingegebenen Werk

510 Ausprobiert werden kann DeepDream z. B. unkompliziert auf https://deepai.org/ machine-learning-model/deepdream (Stand: 22.02.2021). 
unterscheiden. Da der Benutzer durch die Auswahl von Parametern jedoch einen Gestaltungsspielraum genutzt hätte, wäre er auch in diesem Fall als Urheber anzuerkennen.

Es sind Anwendungen von DeepDream denkbar, die von den Entwicklern so voreingestellt sind, dass das Ergebnis sehr stark von den Eingabedaten abweicht. Dann könnte dem Benutzer die Urheberschaft am neu entstandenen Ergebnis evtl. abzusprechen sein. In einem solchen Fall wäre jedoch mangels aktiver Beteiligung auch den Entwicklern kein Urheberschutz zuzugestehen, weshalb der Benutzer mit dem dann urheberrechtsfreien Output beliebig verfahren könnte.

\section{Google Bach Doodle}

Die Webanwendung, die Google zu Ehren Johann Sebastian Bachs zur Verfügung gestellt hat, ${ }^{511}$ erlaubt es dem Benutzer, eine zwei Takte umfassende Melodie einzugeben. Auf Knopfdruck ,erfindet“ die Anwendung unter Verwendung des Coconet-ML-Modells ${ }^{512}$ anschließend eine Begleitstimme im musikalischen Stil von Bach. Das Ergebnis kann anschließend geteilt und auch als Audiodatei auf dem eigenen Computer gespeichert werden.

Ein Benutzer könnte sich nun fragen, ob er als Urheber des Ergebnisses anzusehen ist, sofern das Ergebnis als schutzfähig zu beurteilen wäre. Unter Verwendung des vorgestellten Schemas ergibt sich folgendes: (1) es kommen mehrere Urheber in Betracht, da die Melodie durch den Benutzer eingebracht wurde. Das führt über (2) zu (2.1.) - ein (Dritter) Benutzer gibt Input nach Start des Gesamtsystems in Form einer Melodie, und zu dieser Melodie wird eine Begleitung harmonisiert. Fraglich könnte nun sein, ob die einzugebende Melodie eher Parametern oder einem „komplexen Werk“ gleicht (2.2.). Eine Einordnung als Musikstück und damit als Werk liegt hier nahe, und es handelt sich auch nicht um Werte, mit denen das System eingestellt wird. Es ist daher (2.4.) zu überprüfen, ob ein hinreichender Zusammenhang zwischen der Melodie und dem ausgegebenen Ergebnis zu erkennen ist. Da lediglich eine Begleitung hinzugefügt wird, die aber in der Regel wesentlich von der Melodie bestimmt und geprägt sein wird, ist ein hinreichender $\mathrm{Zu}$ -

511 Vgl. dazu https://www.google.com/doodles/celebrating-johann-sebastian-bach (Stand: 22.02.2021).

512 https://magenta.tensorflow.org/coconet (Stand: 22.02.2021); Huang et al., Counterpoint by Convolution. 
sammenhang anzunehmen. Es spricht daher Vieles dafür, die Urheberschaft beim Einsatz des Google Bach Doodle beim Benutzer zu verorten und die Anwendung damit als (wenn auch potentes) Werkzeug einzustufen. Ob die Melodie hinreichend individuell ist, um als schutzfähig zu gelten, ist eine Einzelfrage und fallbezogen zu klären.

\section{Edmond de Belamy}

Sehr viel Aufmerksamkeit wurde generativen ML-Systemen insbesondere im Oktober 2018 zuteil, als das Gemälde mit dem Titel „Edmond de Belamy“ für viel Geld versteigert wurde. ${ }^{513}$ Der Versteigerungserlös ging an das Künstlerkollektiv Obvious, die angaben, dass das Gemälde Ergebnis einer kreativen Leistung - bzw. einer Kreativität simulierenden Leistung - eines KI-Systems sei. ${ }^{514}$ Kurios war insbesondere auch die Hintergrundgeschichte: Das eingesetzte GAN-Modell wurde maßgeblich von dem amerikanischen Studenten Robbie Barrat entwickelt. Kurz ging es in der anschließenden Diskussion daher auch um Urheberrechte. Allerdings hatte Barrat sein System unter einer sehr liberalen Open Source-Lizenz zur Verfügung gestellt ${ }^{515}$ und wohl schon deswegen keinerlei Anstalten gemacht, etwaige Urheberrechte an dem Bild durchzusetzen. Nichtsdestotrotz enthält das GitHub-Repository von Robbie Barrat inzwischen einen Lizenzhinweis, ${ }^{516}$ der es verbietet, die Erzeugnisse kommerziell zu verwerten. ${ }^{517}$

513 Der Versteigerungserlös betrug umgerechnet ca. $380.500 €$, vgl. Kurzbericht auf ZEIT ONLINE vom 26. Oktober 2018, https://www.zeit.de/kultur/kunst/201810/kuenstliche-intelligenz-versteigerung-gemaelde-algorithmus-christie-sauktionshaus (Stand: 22.02.2021).

$514 \mathrm{Vgl}$. Bericht auf https://www.christies.com/features/A-collaboration-between-twoartists-one-human-one-a-machine-9332-1.aspx (Stand: 22.02.2021), „[...] we found that portraits provided the best way to illustrate our point, which is that algorithms are able to emulate creativity.“

515 Das Projekt ist abrufbar unter https://github.com/robbiebarrat/art-DCGAN (Stand: 22.02.2021).

516 Siehe https://github.com/robbiebarrat/art-DCGAN/blob/master/LICENSE.md (Stand: 22.02.2021).

$517 \mathrm{Ob}$ diese Klausel einer AGB-Kontrolle standhielte, ist jedoch äußerst fraglich - je nach Einsatz des Modells ist das Erzeugnis nach dem hier Vertretenen entweder als gemeinfrei oder als Werk des Verwenders des Modells einzuordnen, womit diese Klausel vorgreifend Urheberrechte beschneiden würde. 
Fraglich ist jedoch, wie die Situation ohne Vorliegen irgendeiner Lizenz zu beurteilen gewesen wäre. ${ }^{518}$ Frage 1 des Schemas kann eindeutig verneint werden: Für die vorzunehmende Analyse ist Obvious Entwickler des Gesamtsystems und Barrat Entwickler des Fremdmodells. Das System war (nach alldem, was darüber bekannt ist) auch nicht inputabhängig. Allerdings wurde ein fremdes ML-Modell eingesetzt (3.). Dieses war vortrainiert (Obvious verwendete ein von Barrat bereitgestelltes vortrainiertes Modell). Ob das Modell umtrainiert wurde, ist nicht bekannt - allerdings gelang es einem neuseeländischen KI-Künstler namens Tom White, unter Einsatz des unveränderten Codes von Barrat, dem Obvious-Ergebnis sehr ähnliche Portraits zu erzeugen, ${ }^{519}$ was wohl dafür spricht, dass das Modell weder umtrainiert wurde (3.2.) noch dass wesentliche Änderungen an dem Fremdmodell vorgenommen wurden.

Die folgenden Fragen 3.4. und 3.5. können mangels entsprechender Informationen nicht beantwortet werden, was aber dahinstehen kann, denn: Das Fremdmodell erzeugte wohl mehrere Ergebnisse (bzw. wurde mehrfach eingesetzt, um eine Vielzahl an Ergebnissen zu produzieren) (3.6.) aus denen dann die „Familie Belamy“ zusammengestellt wurde. Die Auswahlentscheidungen - insbesondere auch im Hinblick auf das Portrait des versteigerten „Edmond de Belamy“ - wurden durch Obvious getroffen und sofern $\mathrm{Ob}$ vious tatsächlich als Entwickler des Gesamtsystems betrachtet werden kann (davon ist auszugehen, wenn die Diskussionen auf GitHub berücksichtigt werden - demzufolge mussten wohl zumindest noch kleinere Anpassungen vorgenommen werden, um das System einsetzen zu können ${ }^{520}$ ) hat auch „,der Entwickler das System selbst eingesetzt" (5.). Damit wäre zu dem Ergebnis zu kommen, dass eine etwaige Urheberschaft an dem versteigerten Gemälde bei Obvious zu suchen ist. Wenn der von Obvious geleistete Anteil an der Erzeugung der Ergebnisse nicht ausreichen sollte, um eine persönliche geistige Schöpfung anzunehmen, hätte das die Gemeinfreiheit des Gemäldes zur Folge.

Das Ergebnis der Prüfung hätte jedenfalls nicht dazu geführt, dass Obvious das Gemälde nicht hätte versteigern lassen dürfen. Wenngleich das für Barrat, der wohl einen maßgeblichen Teil zur Entstehung von „Belamy“ beigetragen hat, ungerecht erscheinen mag: Der Entwickler hat immer die Möglichkeit,

518 Abgesehen von den Fragestellungen die sich dann unter Umständen aufgrund einer Vervielfältigung des Codes stellen könnten.

519 Vincent, How Three French Students Used Borrowed Code to Put the First AI Portrait in Christie's (theverge.com vom 23.10.2018).

520 https://github.com/robbiebarrat/art-DCGAN/issues/3 (Stand: 22.02.2021). 
sein Modell im Voraus zu monetarisieren. Entscheidet er sich wie in diesem Fall dafür, sein Projekt unter einer Open Source-Lizenz bereitzustellen, muss er damit rechnen, dass andere sein Programm verwenden und daraus ggf. Gewinn schlagen.

\section{GPT-3}

„GPT-3“ steht für Generative Pre-Trained Transformer ${ }^{521}$ und bezeichnet ein System, das - inzwischen in der dritten Generation - im Bereich der „,natürlichen Sprachverarbeitung“, bzw. Natural Language Processing (NLP) beeindruckende Ergebnisse erzielt. Das System, dem für den Lernprozess 175 Milliarden Parameter zur Verfügung stehen, ${ }^{522}$ kann schon aus wenigen Worten eine Aufgabenstellung erkennen und etwa eine Geschichte fortsetzen ${ }^{523}$ oder aus der textuellen Beschreibung einer Website den entsprechenden Code erzeugen. ${ }^{524}$ Besonders an dem System ist, dass der Benutzer die Aufgabe, die das System erfüllen soll, definieren kann.

Das Modell wurde auf sehr umfassenden Sprachtrainingsdaten trainiert, und kann eine neue Aufgabe anhand weniger Beispiele erlernen. ${ }^{525}$ Der KIKünstler Mario Klingemann berichtet auf Twitter, dem Modell lediglich den Titel einer Geschichte, den Namen des Autors, in dessen Schreibstil der Text verfasst sein soll, und ein Anfangswort (,Es“) vorgegeben zu haben, woraufhin das System einen vollständigen Aufsatz produzierte. ${ }^{526}$

Hier fällt es deutlich schwerer, dem Inputgeber die Urheberschaft an dem erzeugten Text zuzuschreiben. Der Inhalt und der Stil werden zwar grob vor-

521 https://openai.com/blog/better-language-models/\#sample4 (Stand: 22.02.2021).

522 Brown et al., Language Models are Few-Shot Learners, S. 1.

523 Einige Beispiele sind hier zu finden: https://openai.com/blog/better-languagemodels/\#sample1 (Stand: 22.02.2021); vgl. außerdem für eine Beschreibung des Projekts und des dahinterstehenden Geschäftsmodells https://openai.com/blog/openaiapi/ (Stand: 22.02.2021).

524 So könnte das System aus der Anweisung „Eine Webseite mit einem blauen Button, auf dem KI steht" den für die Anzeige im Browser erforderlichen HTML-Code erzeugen, vgl. für einige Code-Beispiele hier: https://analyticsindiamag.com/openai-gpt-3-code-generator-app-building/ (Stand: 22.02.2021).

525 Brown et al., Language Models are Few-Shot Learners, S. 5.

526 Der erzeugte Aufsatztext ist online einsehbar unter https://drive.google.com/file/ d/1qtPa1cGgzTCaGHULvZIQMC03bk2G-YVB/view (Stand: 22.02.2021), vgl. außerdem den Beitrag von Klingemann auf Twitter, https://twitter.com/quasimondo/ status/1284509525500989445 (Stand: 22.02.2021). 
gegeben, aber die Umsetzung kann allenfalls den Gedankeninhalt haben „Text im Stil von XY unter dem Titel YZ“. Auf verwendete Redewendungen, Spannungsaufbau, Struktur des Textes, bildliche Sprache etc. kann kein Einfluss genommen werden, der Gestaltungsspielraum ist damit stark eingeschränkt (vgl. 2.3. aus dem vorgeschlagenen Schema). Damit liegt es nahe, Gemeinfreiheit festzustellen. Die Eigenart des Systems, aus Beispielen zu lernen, bietet jedoch noch einen weiteren Ansatz: Damit GPT-3 solche Ergebnisse erzeugt, muss es zunächst anhand einer Aufgabenstellung und Beispielen lernen, diese durchzuführen. Die Aufgabenstellung und Bereitstellung von zur Erfüllung der Aufgabe dienlichen Beispielen erfolgt durch den (selben) Benutzer. Dadurch wird der Benutzer zum Lehrer - und gibt damit wesentlich die Richtung vor, in die die Ergebnisse des Systems zielen. Das Ergebnis ist also letztendlich doch die Verkörperung einer Idee, die dementsprechend dem Urheber zuzurechnen ist.

Dem könnte zu entgegnen sein, dass in einem traditionellen Lehrer-SchülerVerhältnis der Urheber des Werkes (sofern der Lehrer die Durchführung nicht vorgegeben hat bzw. sofern noch ausreichend Spielraum für den Schüler besteht, eigenschöpferisch tätig zu sein) in der Regel der Schüler sein wird. ${ }^{527}$ Hier ist jedoch zu berücksichtigen, dass eine Urheberschaft des „Schülers“ in diesem Szenario nicht infrage kommt. Es wird einzig und allein auf den eigenschöpferischen Beitrag des „Lehrers“ ankommen. Sofern dieser eine konkrete Idee hat, hinreichend Gestaltungsspielraum besteht und diese Idee des Benutzers schlussendlich verkörpert wird, muss dieser auch als Urheber Schutz für das Ergebnis genießen können.

\section{Zusammenfassung}

In diesem Kapitel wurde die Schwierigkeit aufgezeigt, aufgrund der zahlreichen, an der Entwicklung eines ML-Modells sowie der Erzeugung von Output beteiligten Personen einen potenziellen Urheber zu ermitteln. Dieser Herausforderung wurde mit dem Vorschlag eines Flussdiagramms begegnet, das anhand von Fragen die Feststellung eines Urhebers für verschiedene Architekturen von Modellen erleichtern soll. Der Einsatz dieses Flussdiagramms wurde sodann an vier aktuellen Beispielen generativer Machine Learning-Modelle demonstriert. Für die Anwendung in der Praxis sollte dieses Flussdiagramm auch durch dieser Arbeit fremde Experten evaluiert

527 Wandtke/Bullinger-Thum, PK UrhR, § 7 Rn. 21 ff.. 
werden. Sodann bietet es sich an, das Diagramm der interessierten Öffentlichkeit zur Verfügung zur stellen, denkbar ist dabei auch eine Umwandlung in eine Webanwendung, die den Nutzer im Frage-Antwort-Format zu einem Ergebnis führt. 


\section{$\S 11$ Ergebnis des vierten Teils}

In diesem Teil erfolgte zunächst eine Analyse der Prüfungsschritte einer persönlichen geistigen Schöpfung in Bezug auf etwaige Herausforderungen beim Einsatz generativer ML-Modelle. Dabei stellte sich heraus, dass insbesondere das Merkmal der menschlich-gestalterischen Handlung besonderer Aufmerksamkeit bedarf, wenn solche Modelle eingesetzt werden: Hier war ein Weg zu finden, einen Zusammenhang zwischen der Handlung des Entwicklers oder Bedieners und dem entstehenden „Werk“ herzustellen. Dazu wurde ein Lösungsvorschlag unter Anwendung eines Dreischritts entwickelt (Auswahl der Rahmenbedingungen, aktive Begrenzung des Gestaltungsspielraums und Treffen einer Auswahlentscheidung). ${ }^{528}$

Der These, mithilfe oder ,durch“ Machine Learning-Modelle erzeugte Ergebnisse seien einem urheberrechtlichen Schutz nicht zugänglich, ist also nicht uneingeschränkt zuzustimmen. Vielmehr kommt es zu dieser Schutzlosigkeit nur in Ausnahmefällen: wenn kein Urheber gefunden werden kann bzw. weil die erforderlichen Merkmale einer menschlich-gestalterischen Handlung nicht in einer Person vorliegen, oder - bisher scheinbar rein fiktiv $^{529}$ - im Fall eines absolut autonom agierenden Systems. Für die Mehrzahl der Fälle kann hingegen - mit Unterstützung des vorgestellten Flussdiagramms - zumindest ein Urheber gefunden und das Vorliegen einer menschlich-gestalterischen Handlung angenommen werden. ${ }^{530}$

Eine Einzelfallbetrachtung ist dann nur noch für die übrigen Merkmale einer persönlichen geistigen Schöpfung - geistiger Gehalt, Wahrnehmbarkeit, Individualität - erforderlich, wobei sich hierbei keine Besonderheiten gegenüber ,klassisch“ erzeugten Werke ergeben dürften. Es verbietet sich folglich eine kategorische Verbannung der ML-Modelle aus dem Urheberrecht. Stattdessen ist der technische Fortschritt zu würdigen, und mit Beruhigung festzustellen, dass das Urheberrecht de lege lata gute Möglichkeiten bietet, auch neue Technologien im Rahmen der urheberrechtlichen Bewertung zu berücksichtigen.

Es mag Fälle geben, in denen es ungerecht erscheint, dem Entwickler des Systems keine Urheberrechte zuzusprechen, weil er das System zur

528 S. oben d).

529 Auf diesbezügliche Möglichkeiten wird unten in $\S 13$ eingegangen.

530 S. oben Abbildung 10.1 in $§ 10$ B.. 
Erzeugung des betreffenden Werkes nicht selber aktiv eingesetzt hat. Dem ist jedoch zu entgegnen, dass es dem Entwickler freisteht, sein Modell das selbst urheberrechtlich umfassend geschützt ist, vgl. § 7 -im Voraus zu monetarisieren, indem er es zum Beispiel kostenpflichtig zur Verfügung stellt.

Noch offen, aber für das geltende Urheberrecht erst einmal unerheblich, ist die Frage, in welchem Fall wirklich von „kreativer künstlicher Intelligenz“ gesprochen werden könnte. Darauf wird im folgenden Kapitel einzugehen sein. 


\title{
Fünfter Teil \\ Computational Creativity - Kreative Künstliche Intelligenz?
}

\begin{abstract}
„Die Vorstellung hochintelligenter Maschinen nur als Science-Fiction abzutun, ist verführerisch, doch das wäre ein Fehler - womöglich überhaupt der schlimmste Fehler, den wir begehen könnten.“ (Stephen Hawking) ${ }^{531}$
\end{abstract}

Nach der in dieser Arbeit bis zu diesem Punkt erfolgten realitätsbezogenen Analyse des Status Quo unter der Prämisse der doch eher schwammigen Begriffe der Kreativität und der Intelligenz liegt der Fokus im letzten Teil der Arbeit auf dem Hinterfragen ebenjener Begriffe. Was macht Kreativität und Intelligenz aus? Was muss Technik können, damit Menschen sie als intelligent und kreativ akzeptieren? Freilich ist dafür Voraussetzung, dass sich der Leser von der Vorstellung löst, dass Kreativität und Intelligenz Teil der exklusiven Domäne der Menschen sind. Oder sind solche Szenarien völlig undenkbar?

Selbst wenn letzteres der Fall wäre, kann eine Auseinandersetzung mit der Materie doch dazu beitragen, dass der Mensch verstärkt seine exklusiven Qualitäten wahrnimmt.

Wie sehr die Grenzen zwischen „echter“ und „künstlicher“ Intelligenz verschwimmen können verdeutlicht folgendes Gedankenexperiment:

Eine Person - Alice - leidet an einer Krankheit, die nach und nach ihre Hirnzellen schädigt. Alice hat Glück und wird Teil einer experimentellen Studie, in deren Rahmen künstliche Hirnzellen getestet werden. Um Alices Hirnfunktion zu erhalten, werden also die bisher geschädigten Hirnzellen durch künstliche Hirnzellen ersetzt, sodass Alice jetzt nur noch zu 90 Prozent menschliche Hirnzellen besitzt. Allerdings schreitet die Krankheit fort, und weitere Hirnzellen sterben ab. Alice lässt diese wiederum durch künstliche Hirnzellen ersetzen. Dieser Prozess wiederholt sich für einige Zeit. Ein paar Jahre später sind alle menschlichen Hirnzellen in Alices Kopf durch künstliche ersetzt. ${ }^{532}$

Ist Alice jetzt noch ein Mensch? Kann Alice noch ,,natürlich intelligent" sein? Macht es einen Unterschied, ob die künstlichen Hirnzellen organischer oder - gleich dem Bild des „Positronenhirns“ in diversen

531 Hawking, Kurze Antworten auf große Fragen, S. 209.

532 Gedankenexperiment in Anlehnung an das Theseus-Paradoxon, das sich eigentlich vielmehr mit der Identität von Objekten befasst als mit der hiesigen Frage nach Intelligenz, aber die Problematik doch eindrücklich beleuchtet, vgl. z. B. Plutarch/Eyth (Übers.), Theseus und Valerius Publicola, 23. Kapitel, S. 29 f.. 
Science-Fiction-Romanen - elektronischer Natur sind? Und, um zum Urheberrecht zurückzukehren: Würden Alice, wenn sie ein Bild malen würde, daran Urheberrechte zustehen?

In diesem Teil wird zur Annäherung an Antworten auf diese Fragen zunächst eine Erläuterung der Begriffe „Kreativität“ und „Intelligenz“ und deren Zusammenhang erfolgen. ${ }^{533} \mathrm{Zu}$ erwarten ist, dass das Verständnis der beiden Begriffe maßgeblich die Erwartungen an künstliche Kreativität und künstliche Intelligenz beeinflusst. Eingegangen wird auch auf Berührungspunkte von Kreativität und Intelligenz mit dem Recht. Sodann wird das Feld der „Computational Creativity“ anhand einiger Umsetzungsversuche vorgestellt. Es folgt eine kritische und kreative urheberrechtliche Überlegung in Bezug auf Computational Creativity, in deren Rahmen bisher zu verzeichnenden Erfolge einer kurzen urheberrechtlichen Analyse unterzogen werden.

533 Die Qualitäten des „Menschseins“, die anhand des Gedankenexperimentes hinterfragt werden könnten, liegen außerhalb des Betrachtungsgegenstandes. 


\section{$\S 12$ Intelligenz, Kreativität und ihre Schnittstellen zueinander und zum Recht}

\section{A. Intelligenz}

„Intelligenz ist eine sehr allgemeine geistige Kapazität, die - unter anderem - die Fähigkeit zum schlussfolgernden Denken, zum Planen, zur Problemlösung, zum abstrakten Denken, zum Verständnis komplexer Ideen, zum schnellen Lernen und zum Lernen aus Erfahrung umfasst. Es ist nicht reines Bücherwissen, keine enge akademische Spezialbildung, keine Testerfahrung. Vielmehr reflektiert Intelligenz ein breiteres und tieferes Vermögen, unsere Umwelt zu verstehen, zu kapieren, 'Sinn in Dingen zu erkennen' oder 'herauszubekommen', was zu tun ist. “534

- so definiert Gottfredson „Intelligenz“, und gibt damit das Ergebnis eines „Mainstream-Statements“ wieder, das 1994 von 52 Experten unterschrieben und veröffentlicht wurde. Ein Bezug zum Menschen könnte in dieser abstrakten Definition allenfalls implizit hergestellt werden: Gottfredson nennt als Gegenbeispiele „Bücherwissen“ und ,akademische Spezialbildung“, und durch die Verwendung des Personalpronomens ,unsere Umwelt“ stellt sie die Qualität des Subjekts auf eine Stufe mit dem - menschlichen - Autor bzw. Leser. Und dennoch ist die Definition so breit gehalten, dass sie auch auf andere Subjekte anwendbar sein könnte - etwa ein Tier oder auch eine Maschine. Fraglich ist, ob das dargelegte Verständnis von Intelligenz lediglich beschreibende Funktion hat, oder ob auch umgekehrt das Vorliegen der beschriebenen Merkmale einen Schluss auf Intelligenz zulässt. Könnte also eine Maschine, die die Fähigkeit zum schlussfolgernden Denken, zum Planen, zur Problemlösung, zum abstrakten Denken, zum Verständnis komplexer Ideen, zum schnellen Lernen und zum Lernen aus Erfahrung besitzt, als intelligent bezeichnet werden? ${ }^{535}$

Zentrale Begriffe scheinen hier das Denken und das Lernen zu sein. Dass eine Maschine nach Schlüssen agieren kann, ist schon seit langer Zeit in Form von Wenn-Dann-Anweisungen implementiert. Es ließe sich sogar sagen, dass

534 Gottfredson, Intelligence 24 Nr. 1 1997, S. 13 (eigene Übersetzung).

535 Hier kommt oft wieder das Paradox zum Zuge, dass wir, was wir an Maschinenhandeln nachvollziehen können, häufig nicht als intelligent anerkennen. Haben wir Angst davor, dass die Differenz zwischen der künstlichen und der echten Intelligenz zu klein wird oder gar gegen Null divergiert? 
Maschinen wahre Meister im Schlussfolgern sein müssen, denn - zumindest die „klassischen“ Systeme - folgen in der Regel strikt Anweisungen und wählen die durchzuführende Handlung nach der Analyse von Entscheidungskriterien aus. Wenn ein Computer für eine Berechnung länger als nur einen Augenblick Zeit benötigt, ist der Mensch verleitet, das mit einem ,der Computer denkt noch“ abzutun. Aber meint der Mensch damit Denken im Sinne des oben gemeinten Denkens? Oder assoziieren wir Denken zwingend mit einem menschlichen oder doch zumindest biologischen Gehirn?

Nicht in der Beschreibung von Gottfredson enthalten sind die Komponenten des autonomen Handelns, des eigenständigen Denkens bzw. des freien Willens, die intuitiv auch mit dem Begriff der Intelligenz assoziiert werden könnten.

Diese Diskussion über die Unterschiede menschlicher und versuchtkünstlicher Intelligenz ließen sich noch beliebig weiterspinnen. Im Zentrum dieses Abschnitts der Arbeit soll jedoch keine Grundsatzdiskussion über die Konzepte, die Wahrnehmung und das Hinterfragen von Intelligenz stehen, sondern letztendlich die Idee der „Computational Creativity“ und damit die Frage, wie Kreativität - als wesentliche Komponente der Intelligenz künstlich nachgebildet und eingesetzt werden kann.

\section{Definition der Intelligenz?}

Auf der Suche nach einer allgemein gültigen und akzeptierten Definition des Begriffs der Intelligenz wird schnell die Aussichtslosigkeit dieses Unterfangens klar. Die Versuche, Intelligenz eindeutig zu definieren, sind so mannigfaltig wie die Zahl der Forscher groß ist, die sich dessen bisher angenommen haben. ${ }^{536}$ Vieles spricht dafür, dass es nicht die eine Intelligenz gibt, sondern verschiedene Bereiche existieren, innerhalb derer intelligentes oder weniger intelligentes Verhalten festgestellt werden kann. Dazu gehören etwa das Problemlösen, wissensbasierte Fähigkeiten sowie soziale und praktische Fähigkeiten ${ }^{537}$ aber auch Aspekte wie der Orientierungssinn, emotionale Intelligenz und Musikalität. Diese Vielfalt möglicher Erscheinungsformen wird auch in der oben wiedergegebenen Definition von Gottfredson deutlich.

536 So auch die American Psychological Association, vgl. Neisser et al., American Psychologist 51 Nr. 2 1996, S. 77: ,[...] when two dozen prominent theorists were recently asked to define intelligence, they gave two dozen somewhat different definitions".

537 Sternberg, Beyond IQ, S. 129. 
Es erscheint daher fraglich, ob es sinnvoll ist, das Konzept der „Intelligenz“ zunächst exakt erfassen zu wollen, bevor die Nachbildung derselben versucht wird. Andererseits macht es diese schwammige Definition umso schwieriger, ein Nachbildungsergebnis mit dem „Original“ der Intelligenz zu vergleichen, um den Fortschritt zu evaluieren. Fürs erste scheint Intelligenz ein Konzept zu bleiben, das erkannt wird, sobald es vorliegt, aber nicht abschließend beschrieben werden kann.

An dieser Stelle wird daher auf die Festlegung auf eine Definition bewusst verzichtet. Da die Arbeit aber später versuchen will, die Phänomene der Intelligenz und der Kreativität einer rechtlichen Betrachtung zugänglich zu machen, liegt es nahe, sich im Folgenden mit juristischen Berührungspunkten dieser Begriffe etwas näher zu befassen.

\section{Relevanz der Intelligenz im Recht}

Auch im Recht spielt die Intelligenz keine unbedeutende Rolle. Anknüpfungspunkte für Intelligenz als Voraussetzung bestimmter Rechtsfolgen finden sich beispielsweise etwa im Zivilrecht im Kontext der Geschäftsfähigkeit und im Strafrecht in Bezug auf bestimmte Aspekte der Schuldunfähigkeit, während das Urheberrecht an Realakte unabhängig von Fähigkeiten anknüpft. Die Betrachtung der Relevanz der Intelligenz im Urheberrecht, also dem zentralen Untersuchungsgegenstand dieser Arbeit, erfolgt im Rahmen einer gesonderten Betrachtung an späterer Stelle. ${ }^{538}$

\section{Zivilrecht}

Im Zivilrecht spielt die Abgabe von Willenserklärungen eine wesentliche Rolle, zu denken ist etwa an die Erforderlichkeit von Willenserklärungen für das Zustandekommen eines Kaufvertrags. Voraussetzung für die Wirksamkeit einer Willenserklärung ist dabei stets die (zumindest beschränkte) Geschäftsfähigkeit ( $\S 104$ ff. BGB) im Sinne einer „Fähigkeit rechtsfähiger Menschen (also nur natürlicher Personen), allgemein zulässige Rechtsgeschäfte selbständig vollwirksam vornehmen zu können“. ${ }^{539}$ Dies soll Personen vor möglichen nachteiligen Wirkungen ihrer Erklärungen schützen, die „nicht

538 S. dazu unten $\S 12$ C.II..

539 MüKo-Spickhoff, BGB, § 104 Rn. 2. 
zur eigenverantwortlichen Willensbildung in der Lage sind und nicht die Fähigkeit besitzen, für ihre eigene Person zu sorgen und die Folgen ihres Verhaltens zu übersehen“540 - diese Umschreibung bezieht sich nicht zuletzt auf kognitive Fähigkeiten und damit insbesondere unter Verwendung der weiten Definition auch auf Intelligenz. Wenn (z. B. gerichtliche) Zweifel an der Geschäftsfähigkeit einer Person bestehen, wird zur Begutachtung ein Arzt herangezogen, ${ }^{541}$ dessen Aufgabe dann darin besteht, die Geschäftsfähigkeit beeinträchtigende Faktoren zu evaluieren, wie zum Beispiel psychische Erkrankungen, Demenz und Intelligenzminderungen (im Sinne der durch IQ-Tests messbaren Intelligenz ${ }^{542}$ ). Es wird dabei von der vollen Geschäftsfähigkeit ausgegangen, die Intelligenz also allen Menschen grundsätzlich unterstellt, sodass die Beweislast denjenigen trifft, der die Geschäftsfähigkeit anzweifelt. $^{543}$

Bemerkenswert ist, dass keine Definition eines Idealzustandes des intelligenten Menschen erfolgt, sondern fallbezogen und ergebnisorientiert Situationen vermieden werden sollen, die Nachteile für einen oder mehrere Beteiligte mit sich bringen.

\section{Strafrecht}

Ähnliches gilt im Strafrecht in Bezug auf die Schuldunfähigkeit; § 20 StGB besagt:

„Ohne Schuld handelt, wer bei Begehung der Tat wegen einer krankhaften seelischen Störung, wegen einer tiefgreifenden Bewusstseinsstörung oder wegen Schwachsinns oder einer schweren anderen seelischen Abartigkeit unfähig ist, das Unrecht der Tat einzusehen oder nach dieser Einsicht zu handeln.“

Greift man auf den Teil in Gottfredsons Definition zurück, welche sich mit der praktischen Orientiertheit (,Sinn in den Dingen erkennen“, „herausbekommen, was zu tun ist") als Merkmal der Intelligenz befasst, so scheint $\S 20$ StGB zumindest teilweise auch deren Abwesenheit als Tatbestandsmerkmal zu meinen: Reicht die Geisteskraft zur Erkennung oder Vermeidung des Unrechts nicht aus, so fehlt es sicher nicht selten am grundsätzlichen Vermögen, die Notwendigkeit des „richtigen“ Handelns zu erkennen. Auch

540 MüKo-Spickhoff, BGB, Vor § 104 Rn. 6.

541 Dreßing et al., Der Nervenarzt 85 Nr. 11 2014, S. 1142; MüKo-Spickhoff, BGB, $\S 104$ Rn. 31.

542 Dreßing et al., Der Nervenarzt 85 Nr. 11 2014, S. 1446 ff..

543 MüKo-Spickhoff, BGB, § 140 Rn. 3, 29. 
im Strafrecht ist also ein Zustand, dessen Eigenschaften möglicherweise der Intelligenz zugeordnet werden könnten, Voraussetzung dafür, dass ein Täter schuldfähig und damit bestrafbar ist. Ein Ähnliches ergibt ein Blick auf $\S 17$ S.1 StGB:

„Fehlt dem Täter bei der Begehung der Tat die Einsicht, Unrecht zu tun, so handelt er ohne Schuld, wenn er diesen Irrtum nicht vermeiden konnte.“

(Auch) hier wird die Intelligenz im Sinne einer Einsichtsfähigkeit herangezogen, um die Verantwortung des Täters für die begangene Tat zu ermessen.

\section{Folgerung}

Auch wenn im Rahmen dieser Untersuchung auf eine konkrete Definition des Begriffs der Intelligenz verzichtet wird, lassen sich zumindest doch auch im Ansatz von Gottfredson zwei wesentliche Komponenten des Konzeptes der Intelligenz feststellen: Zum einen wird die Fähigkeit, zu lernen, und Zusammenhänge zu erkennen, verlangt, die sich in eine ,analytische Komponente“ fassen lassen. Zum anderen wird auch die Problemlösung grundsätzlich der Intelligenz zugeschrieben, also eine „konstruktive Komponente“ der Intelligenz vorausgesetzt. Dass Letztere insbesondere im Rahmen der Kreativität von zentraler Bedeutung ist, wird sich später noch zeigen. ${ }^{544}$

Das Recht kommt ohne eine spezifische Definition von Intelligenz aus, obgleich es sich immer wieder auf Merkmale bezieht, die dem Bedeutungsfeld dieses Begriffs angehören. Dies dürfte daher rühren, dass die Intelligenz als implizite Eigenschaft des Menschen vorausgesetzt wird, und Rechtsfolgen lediglich an konkrete Defizite der geistigen Leistungsfähigkeit in speziellen Handlungszusammenhängen geknüpft werden.

Intuitiv könnte das tatsächliche Vorliegen von Kreativität den Nachweis von Intelligenz erleichtern, wenn davon ausgegangen wird, dass Kreativität Intelligenz voraussetzt, wobei zu berücksichtigen ist, dass auch der Zusammenhang zwischen Intelligenz und Kreativität noch der Klärung bedarf. Dazu ist allerdings eine Feststellung tatsächlicher Kreativität notwendig, die ihrerseits nach Definition und Kriterien verlangt.

544 Vgl. dazu z. B. d). 


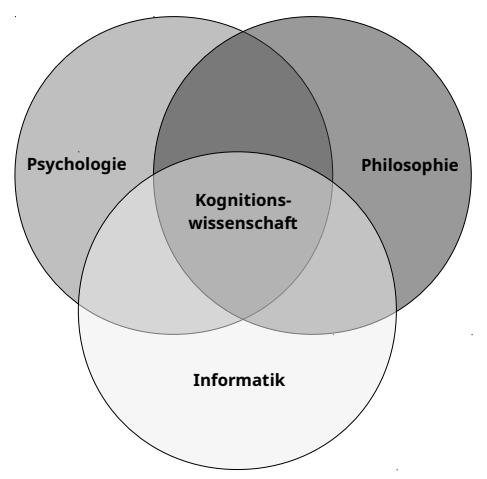

Abbildung 12.1: Kognitionswissenschaft im Kontext. Quelle: eigene Darstellung.

\section{B. Kreativität}

In der Kreativitätsforschung sind verschiedene Schwerpunkte bzw. Richtungen festzustellen, in deren Rahmen sich die Definitionsansätze weiter unterscheiden. Geforscht wird naheliegenderweise auf dem Gebiet der Psychologie, ${ }^{545}$ in der Informatik - hier insbesondere unter den Begriffen des „Creative Computing“, „Computational Creativity“ oder „Artificial Creativity "546 - und in der Philosophie ${ }^{547}$.

Als interdisziplinäre Schnittstellenwissenschaft hat sich die Kognitionswissenschaft herausgebildet, in deren Forschung die Erkenntnisse aus den zuvor genannten Gebieten zusammenfließen (vgl. Abbildung 12.1). ${ }^{548}$ Darüber hinaus beschäftigen sich auch zahlreiche Künstler mit dem Begriff der

545 Insb. Robert J. Sternberg; z. B. Sternberg, The Nature of Creativity; Ders., Handbook of Creativity; Ders., Beyond IQ; Mihály Csíkszentmihályi: Csikszentmihalyi, Society, culture, and person: A systems view of creativity.

546 Vgl. z. B. Aaron Sloman: Sloman, The Creative Universe, aber auch schon Ada Lovelace: Lovelace, Scientific Memoirs 1843.

547 Vgl. z. B. Karl-Heinz Brodbeck: Brodbeck, Zur Philosophie der Kreativität, Yuval Noah Harari: Harari, Homo Deus.

548 Stephan/Walter, Handbuch Kognitionswissenschaft, S. 4, zusätzlich werden noch die Gebiete der Anthropologie, der Linguistik und der Neurowissenschaft erfasst. Für Forschung zum Thema der Kreativität auf dem Gebiet der Kognitionswissenschaft vgl. z. B. Margaret Boden: Boden, Creative Mind; Jon McCormack und Mark d'Inverno: McCormack/d'Inverno, Computers and Creativity. 
Kreativität im technischen Kontext. ${ }^{549}$ Einen sehr umfangreichen und anschaulichen Überblick über technische Aspekte der Implementierung von Kreativität und deren Anwendung in verschiedenen Bereichen der Kunst gibt Miller ${ }^{550}$ der sich auch an eine Kreativitätsdefinition bzw. -kateogrisierung heranwagt. ${ }^{551}$

\section{Definition?}

Wie der Überblick über die an Definitionsversuchen beteiligten Disziplinen erahnen lässt, ist eine präzise und abschließende Beschreibung eines Begriffes der Kreativität bisher nicht bedingungslos konsensfähig. ${ }^{552}$ Es folgt zunächst ein Versuch der etymologischen Herleitung des Begriffs, ${ }^{553}$ anschließend eine Darstellung der verschiedenen Strömungen, die sich um eine Begriffsdefinition bemühen, mit dem Ziel, zumindest ein gewisses Grundverständnis für Kreativität zu ermöglichen. Der Fokus liegt auf den Ansätzen der Kognitionswissenschaft, da hier bereits eine Zusammenführung der verschiedenen beteiligten Disziplinen angestrebt wird. Die Ausführlichkeit der Begriffsuntersuchung ist dem Umstand geschuldet, dass die herauszuarbeitenden Kriterien im Anschluss konsolidiert und weiterverwendet werden sollen.

\section{Etymologischer Ursprung von „Kreativität“}

Der Terminus kreativ wurde, im 19. Jahrhundert übernommen aus dem Englischen (creative), in Deutschland insbesondere seit der Auseinandersetzung mit der US-amerikanischen Kreativitätsforschung Ende der 1960er Jahre populär ${ }^{554}$ und beruht auf dem lateinischen Begriff creare, bzw. creatum (erschaffen, geschaffen). ${ }^{555}$ Damit läge eine Definition nahe, die das Schaffen

549 Z. B. Mario Klingeman: u. a. https://quasimondo.com (Stand: 22.02.2021); Simon Colton: Colton, The Painting Fool u. v. m.

550 Miller, The Artist in the Machine.

551 Vgl. dazu unten d).

552 So auch Boden, AI and Creativity: Contradiction?, S. 3.

553 Mit der Geschichte des Begriffs der Kreativität beschäftigen sich Still/d'Inverno, A History of Creativity for Future AI Research.

554 Brodbeck, Zur Philosophie der Kreativität, S. 15.

555 https://de.wiktionary.org/wiki/kreativ (Stand: 22.02.2021); https://www.duden.de/ rechtschreibung/kreativ (Stand: 22.02.2021). 
von etwas Neuem in den Mittelpunkt stellt, in Bezug auf die hiesige Materie also das „Hervorbringen neuer Ideen“ bezeichnen würde. ${ }^{556}$

\section{Psychologie}

Eine Definition des Kreativitätsbegriffs aus der Psychologie bezeichnet Kreativität als ,original thinking“, also in etwa: eigenes, nicht anderweitig übernommenes Denken, das zu neuen „Produkten“ führt die in ihrem sozialen Kontext Wert haben. ${ }^{557}$

Wohl ebenfalls der Psychologie zuzuordnen ist der Ansatz von Runco und Jaeger, die - wie hier - feststellen, dass es nicht nur nahezu unüberschaubar viele Ansätze der Definition des Kreativitätsbegriffs gibt, sondern auch fast alle Ausarbeitungen zu dem Thema auf Publikationen aus den Achtzigern und Neunzigern des letzten Jahrtausends zurückgreifen (müssen). ${ }^{558}$ Damit kritisieren sie jedoch nicht, dass die Publikationen veraltet seien, sondern weisen vielmehr darauf hin, dass der Kreativitätsbegriff eine viel längere Geschichte hat und Recherchen jenseits der online verfügbaren Medien zu erfolgen haben, um diese Geschichte vollständig zu erfassen. ${ }^{559}$ Für sie ist die „Standarddefinition“ zweiteilig: Kreativität erfordere Originalität einerseits und Effektivität bzw. Zweckmäßigkeit andererseits, wobei die Erforderlichkeit des letzteren Kriteriums zu hinterfragen sei. ${ }^{560}$ Auf der Suche nach dem Ursprung dieser Standarddefinition befinden die Autoren Morris I. Stein ${ }^{561}$ als den Wissenschaftler, der diese Definition zuerst, im Jahr 1953, unzweideutig in einer Veröffentlichung verwendet hat. ${ }^{562}$

Dieser „Standarddefinition“ lässt sich auch das zu Beginn dieses Abschnitts genannte Begriffsverständnis von Lubart und Thornhill-Miller zuordnen, und auch Sternberg schließt sich dieser Auffassung an:

556 Vgl. z. B. Boden/Savigny, Flügel des Geistes, S. 12.

557 Versuch einer möglichst nahen Übersetzung zu ,original thinking that leads to new productions that have value in their social context", Lubart/Thornhill-Miller, Creativity: An Overview of the 7C's of Creative Thought, S. 277.

558 Runco/Jaeger, Creativity Research Journal 24 Nr. 1 2012, S. 92.

559 Dies., Creativity Research Journal 24 Nr. 1 2012, S. 92.

560 Dies., Creativity Research Journal 24 Nr. 1 2012, S. 92.

561 Stein, The Journal of Psychology 36 1953, S. 311.

562 Runco/Jaeger, Creativity Research Journal 24 Nr. 1 2012, S. 94 f.. 
„Kreativität ist die Fähigkeit, Werke zu produzieren die sowohl neu sind (d. h. originell, unerwartet) als auch angemessen (d. h. nützlich, an durch die Aufgabe auferlegte Beschränkungen angepasst). ${ }^{\text {‘563 }}$

Auffällig ist, dass aus Sicht der „Standarddefinition“ die Originalität - also die Ungewöhnlichkeit, Neuheit oder Einzigartigkeit - zwingendes Kriterium (wenn auch nicht hinreichend) für das Vorliegen von Kreativität ist. ${ }^{564}$ Das klingt danach, als ob die „Alltagskreativität“ von dieser „Standarddefinition“ nicht erfasst werden soll - es sei denn, die Originalität ließe sich auch streng auf das Individuum beziehen (sodass sie auch dann vorläge, wenn ein Individuum ein Problem löst, das selbst es in der Art noch nicht vorher gelöst hat). $\mathrm{Zu}$ dieser Thematik finden sich Ansätze in der Kognitionswissenschaft.

\section{Kognitionswissenschaft}

In der Kognitionswissenschaft beschäftigen sich insbesondere Margaret Boden, Jon McCormack und Mark d'Inverno, aber auch Aaron Sloman und Arthur Miller mit dem Thema der Kreativität. Ihre Definitionsansätze werden im Folgenden kurz vorgestellt.

\section{a) Boden: P-Kreativität und H-Kreativität}

\section{Grundsätzlich definiert Boden Kreativität als}

„die Fähigkeit, Ideen oder Artefakte [Werkstücke] hervorzubringen die neu, überraschend und wertvoll sind." 565

Boden unterscheidet in Bezug auf den Aspekt der Neuheit zwischen historischer und psychologischer Kreativität (H-Kreativität und P-Kreativität). ${ }^{566}$ Erstere beschreibt Ideen, die für die gesamte Menschheit neu sind, die also noch kein Mensch zuvor hatte, P-Kreativität bezieht sich hingegen darauf, dass die Idee für das Individuum neu ist. ${ }^{567} \mathrm{H}$-Kreativität beinhaltet denklogisch immer P-Kreativität, andersherum kann es jedoch viele P-kreative

563 Sternberg, Handbook of Creativity, S. 3.

564 Runco/Jaeger, Creativity Research Journal 24 Nr. 1 2012, S. 92.

565 Boden, Creative Mind, S. 1.

566 Dies., Creative Mind, S. 43; Dies., AI and Creativity: Contradiction?, S. 4; teilweise auch P-Neuheit und H-Neuheit: Dies., Creativity as a Neuroscientific Mystery, S. 1.

567 Dies., AI and Creativity: Contradiction?, S. 4. 
Einfälle eines Individuums geben, die andere Menschen aber bereits kennen. ${ }^{568}$

Des Weiteren kategorisiert Boden Kreativität in kombinatorische, explorative und transformierende Typen. ${ }^{569}$ Kombinatorische Kreativität dürfte darunter wohl die meistbekannte Variante darstellen: Sie erfasst neue Ideen als Kombination bekannter, bestehender Ideen, dazu zählen etwa Collagen, aber auch verbale, visuelle oder musikalische Analogien. ${ }^{570}$ Explorative forschende - Kreativität bezeichnet die Erzeugung neuer Ideen bzw. Werkstücke unter Anwendung bekannter Regeln oder Konventionen, wie zum Beispiel das Schaffen eines neuen Bildes im impressionistischen Stil. ${ }^{571}$ In die dritte Kategorie fallen solche Ideen, die unter bestehenden Regeln unmöglich sind und nur durch Aufbrechen von Konventionen und Denkregeln entstehen können. ${ }^{572}$

Neben der Kategorisierung stellt Boden fest, dass Kreativität Autonomie, Absicht, Bewertung, Emotion und Bewusstsein erfordert. ${ }^{573}$ Dies dürfte die Hürden für künstliche Kreativität deutlich erhöhen, wie später (§ 13) noch festzustellen sein wird.

\section{b) McCormack/d'Inverno}

Auch Jon McCormack und Mark d'Inverno stellen fest, dass die Definition von Kreativität so mannigfaltig scheint wie die wissenschaftlichen Hintergründe derjenigen, die sie produzieren. ${ }^{574}$ Sie schließen sich im Wesentlichen der Definition von Boden an. ${ }^{575}$

$568 \mathrm{Vgl}$. Boden, AI and Creativity: Contradiction?, S. 4, allerdings wurde dort - vermutlich versehentlich - das Gegenteil behauptet.

569 Dies., Creativity as a Neuroscientific Mystery, S. 3.

570 Dies., Creativity as a Neuroscientific Mystery, S. 3.

571 Dies., Creativity as a Neuroscientific Mystery, S. 3 f..

572 Dies., Creativity as a Neuroscientific Mystery, S. 4; dort auch ein Beispiel für die dritte Kategorie: die Idee, dass manche Kohlenstoffmoleküle hohle Kugeln sein könnten. Dafür muss für möglich erachtet werden, dass Atombindungen nicht lediglich ein- sondern auch dreidimensional erfolgen könnten.

573 Dies., AI and Creativity: Contradiction?, S. 1.

574 McCormack/d'Inverno, On the Future of Computers and Creativity, S. 1.

575 Dies., On the Future of Computers and Creativity, S. 1. 
c) Sloman: Intrinsische, expressive und standardtestbasierte Kreativität

Sloman unterscheidet drei Kategorien computerwissenschaftlicher Kreativitätsforschung, ${ }^{576}$ deren zweite wohl insbesondere die drei Kategorien von Boden erfassen dürfte:

- Intrinsische Kreativität,

- expressive Kreativität (,Talent-Kreativität“) und

- Standard-Test-basierte Kreativität.

Intrinsische Kreativität meint „Alltagskreativität“, die bei Aufgaben zum Einsatz kommt, die kombinatorische Fähigkeiten erfordern, etwa die Fähigkeit, Informationen aus einem Stadtplan zu erfassen und daraus den tatsächlichen Weg zum Ziel abzuleiten. ${ }^{577}$

Expressive Kreativität ist nach Sloman als von Menschen häufig als „,besonders" empfundene Fähigkeiten zu verstehen, dazu gehören das Dichten, Malerei, aber auch mathematische bzw. wissenschaftliche oder technische Kreativität. ${ }^{578}$ Die Grenze zwischen intrinsischer und expressiver Kreativität will Sloman jedoch nicht allzu scharf ziehen. ${ }^{579}$

Die dritte Kategorie der „standardised test-based creativity“ erfasst die Formen der Kreativität, die von „Kreativitätstests“ gemessen werden. ${ }^{580}$ Das Vorkommen der Boden'schen Typen der P- und H-Kreativität vermutet Sloman in all seinen Kategorien. ${ }^{581}$

Für die Umsetzung dieser Kreativitätstypen in maschinellen Prozessen sieht Sloman besondere Herausforderungen auf dem Gebiet der intrinsischen Kreativität. Während Handlungen, die in diese Kategorie fallen, für Menschen in der Regel nicht sonderlich herausfordernd oder sogar intuitiv sein können, fällt es nachgeahmten „Intelligenzen“ deutlich schwerer, intrinsisch kreative Aufgaben zu bewältigen.

In der öffentlichen Wahrnehmung sei hingegen Talentkreativität von Maschinen deutlich präsenter: ${ }^{582}$ es sind bereits zahlreiche Systeme im Umlauf, die bemerkenswerte Ergebnisse erzielen. ${ }^{583}$ Dies mag daran liegen, dass es

576 Sloman, The Creative Universe, S. 24.

577 Ders., The Creative Universe, S. 25.

578 Ders., The Creative Universe, S. 25.

579 Ders., The Creative Universe, S. 25.

580 Ders., The Creative Universe, S. 25 f.

581 Ders., The Creative Universe, S. 26.

582 Ders., The Creative Universe, S. 27.

583 Vgl. auch $\S 10 \mathrm{C}$.. 
für solche Kreativitätshandlungen besonders auf das Ergebnis - im Sinne eines erzeugten Kunstwerks - anzukommen scheint. Oben ( $110 \mathrm{C}$.) wurden bereits einige Machine Learning-Systeme vorgestellt, die der expressiven Kreativität zuzuordnen sind.

\section{d) Miller: Little-c- und Big-C-Kreativität}

Eigentlich betrieben auf den Gebieten der Geschichte und Wissenschaftsgeschichte - nicht aber auf dem der Kognitionswissenschaft - aber aufgrund des sehr interdisziplinären Ansatzes doch auch zu den vorgenannten Theorien passend, ist die diesbezügliche Forschung von Arthur Miller. Dieser schlägt eine ähnliche Einteilung der Kreativität wie Sloman vor: ${ }^{584}$ Er kategorisiert nach „little-c creativity“ und „big-C Creativity“, also in etwa „kreativität“ und „Kreativität“" ${ }^{585}$ im Folgenden c-Kreativität und C-Kreativität. Erstere erfasst „Alltagskreativität“ wie etwa das Entdecken eines neuen Weges zum Arbeitsplatz, letztere bahnbrechende Entdeckungen wie z. B. die Relativitätstheorie. ${ }^{586}$ Nach Miller steht das Problemlösen im Zentrum jeglicher Kreativität. ${ }^{587}$ Seine Arbeitsdefinition des Begriffs lautet daher auch wie folgt:

„Kreativität ist die Produktion neuen Wissens auf der Basis bereits existierenden Wissens durch Problemlösen. “588

Er bezieht sich damit nicht nur auf Kreativität in der Wissenschaft, wie der Fokus auf Problemlösen vermuten lassen könnte: Auch Künstler, Dichter, Schriftsteller und Komponisten sieht er als Problemlöser, die auf vorhandenem Wissen und Regeln aufbauen und daraus neues (Wissen) erschaffen, teilweise auch durch Regelbrüche. ${ }^{589}$

584 Weist aber auch darauf hin, dass eine solche Unterscheidung wohl bereits 1988 getroffen wurde, Miller, The Artist in the Machine, S. 27 und verweist dabei auf Csikszentmihalyi, Society, culture, and person: A systems view of creativity, S. $325 \mathrm{ff}$..

585 Miller, The Artist in the Machine, S. 6.

586 Ders., The Artist in the Machine, S. 6.

587 Er schreibt diesen Fokus auf Problemlösung auch schon Einstein zu, vgl. Ders., The Artist in the Machine, S. 9.

588 Ders., The Artist in the Machine, S. 5; übersetzt aus dem Englischen: „Creativity is the production of new knowledge from already existing knowledge and is accomplished by problem solving".

589 Ders., The Artist in the Machine, S. 5 f.. 


\section{Andere Ansätze}

Eine Definition aus dem Gebiet der Philosophie hingegen beschreibt Kreativität schlicht als „Realisierung von Ideen“.590

Andere konstatieren, dass Kreativität aufgrund ihrer Heterogenität schwierig zu definieren sei: Es sei kaum eine Definition denkbar, die allen Bereichen, in denen Kreativität zu finden ist, gerecht würde..$^{591}$

„Unsere Definitionen von Kreativität [...] sind nichts anderes als akzeptierte Konventionen, und es liegt in der Natur der Kreativität, Konventionen zu beugen und zu untergraben." "592

Zudem wurde beobachtet, dass die Wahrnehmung von Kreativität - und damit auch das, was darunter verstanden wird - sich von einer Kultur zur anderen unterscheidet: Verglichen wurden insbesondere westliche und östliche Wahrnehmungen von Kreativität. Während die östliche (chinesische) Vorstellung von Kreativität zum Beispiel stets auch moralische Aspekte beinhaltet, ist diese Eigenschaft in der westlichen - modernen - Wahrnehmung von Kreativität nicht mehr vorhanden. ${ }^{593}$

\section{Schlussfolgerung: Einführung von Kreativitätsprofilen}

Diese Erkenntnisse bringen diese Arbeit in eine Zwickmühle: eine allgemeingültige Definition aufzustellen, würde bedeuten, das eigentlich uneinschränkbare Feld der Kreativität eingrenzen zu wollen. Keine Definition aufzustellen erschwert hingegen die weitere Vorgehensweise zur Analyse „kreativer" KI-Systeme.

Deswegen wird sich einer „Krücke“ bedient: auf eine Definition des Begriffs der Kreativität wird zunächst verzichtet. Stattdessen wird von den von Boden, Sloman und Miller aufgestellten Kategorien Gebrauch gemacht. Diese Vorgehensweise ist letztlich nicht unähnlich zu dem Ansatz einer großen Gruppe von Machine-Learning-Systemen: Durch Klassifizierung und Erkennen von Gemeinsamkeiten und Unterschieden insbesondere beim unüberwachten Lernen ist es nicht notwendig, die Gruppen (Begriffe) im Voraus zu beschreiben bzw. zu definieren.

590 Brodbeck, Zur Philosophie der Kreativität, S. 6.

591 Veale/Cardoso, Computational Creativity, S. 2.

592 Dies., Computational Creativity, S. 2.

593 Niu/Sternberg, Journal of Theoretical and Philosophical Psychology 26 Nr. 1-2 2006, S. $18 \mathrm{ff}$.. 


\begin{tabular}{|c|c|c|c|c|}
\hline von & \multicolumn{3}{|c|}{ Kategorien } & Bezugspunkt \\
\hline Miller & c-Kreativität & & C-Kreativität & absolute Neuheit \\
\hline Boden & P-Kreativität & H-Kreativität & subjektive Neuheit \\
\hline Boden & $\begin{array}{c}\text { kombinatorische } \\
\text { Kreativität }\end{array}$ & explorative Kreativität & $\begin{array}{c}\text { transformierende } \\
\text { Kreativität }\end{array}$ & Prozess \\
\hline Sloman & $\begin{array}{c}\text { intrinsische Kreativität } \\
\text { „natürliche Kreativität“ }\end{array}$ & & $\begin{array}{c}\text { expressive Kreativität } \\
\text { „Talentkreativität }\end{array}$ & Ergebnis / Sichtbarkeit \\
\hline
\end{tabular}

Abbildung 12.2: Kategorien der Kreativität. Quelle: eigene Darstellung.

Abbildung 12.2 fasst diese Kategorien in einer Tabelle zusammen. $\mathrm{Zu}$ berücksichtigen ist, dass die Anordnung der Kategorien in Spalten keinerlei vertikale, etwa hierarchische, Beziehung andeuten soll. So sind etwa Ergebnisse intrinsischer Kreativität denkbar, die zugleich auf transformierenden Kreativitätsprozessen beruhen und als „,bahnbrechend“ im Sinne der C-Kreativität einzuordnen sind - solch ein Fall dürfte etwa die Entdeckung der Relativitätstheorie gewesen sein. Expressive Kreativität kann sich sowohl als für eine Person neu, als auch für die Geschichte neu herausstellen, genauso kann expressive Kreativität „Alltagsqualität“ haben c-Kreativität, etwa ein Zeitungsartikel - oder aber auch so umwerfend anders sein, dass C-Kreativität anzunehmen ist, wie etwa für einen Weltbestsellerroman. Die Erstellung der Tabelle in Abbildung 12.2 lässt sich vermutlich der c-Kreativität, der P-Kreativität, der kombinatorischen Kreativität und der intrinsischen Kreativität zuordnen.

Das Herstellen einer strikten Hierarchie in diesem Zusammenhang scheint also schon deshalb nicht logisch, weil diese Kategorien - wie in der Spalte rechts dargestellt - jeweils unterschiedliche Bezugspunkte haben. Stattdessen könnte ein Werk anhand dieser vier unterschiedlichen Bezugspunktkategorien auf verschiedene Merkmale von Kreativität analysiert werden.

Denkbar wäre auch die Erstellung eines Kreativitätsprofils für den zu betrachtenden Gegenstand oder auch für ein Schutzrecht. Dafür sind den Bezugspunkten entsprechend nicht nur der Gegenstand selbst, sondern auch der Ersteller und der Prozess der Erstellung zu berücksichtigen, vgl. Abbildung 12.3. Abgebildet sind zur Veranschaulichung zunächst - sehr vereinfacht und ohne Rücksicht auf besondere Anforderungen wie etwa die Technizität im Patentrecht - die Mindestanforderungen für einen möglichen Schutz durch das PatR, ein entsprechendes Profil für das UrhR findet sich in Abbildung 12.5 .

Durch die Zuhilfenahme der Bezugspunkte kann unter Umständen eine Vermittlung zwischen verschiedenen Kreativitätsverständnissen erfolgen. 


\begin{tabular}{|c|c|c|c|c|}
\hline von & \multicolumn{3}{|c|}{ Kategorien } & Bezugspunkt \\
\hline Miller & c-Kreativität & 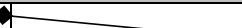 & C-Kreativität & absolute Neuheit \\
\hline Boden & P-Kreativität & & H-Kreativität & subjektive Neuheit \\
\hline Boden & $\begin{array}{l}\text { kombinatorische } \\
\text { Kreativität }\end{array}$ & explorative Kreativität & $\begin{array}{c}\text { transformierende } \\
\text { Kreativität }\end{array}$ & Prozess \\
\hline Sloman & $\begin{array}{l}\text { intrinsische Kreativität } \\
\text { „natürliche Kreativität" }\end{array}$ & & $\begin{array}{l}\text { expressive Kreativität } \\
\text { „Talentkreativität" }\end{array}$ & Ergebnis / Sichtbarkeit \\
\hline
\end{tabular}

Abbildung 12.3: Kreativitätsprofil Patentrecht, Quelle: eigene Darstellung.

Uneinigkeit über den Kreativitätsgehalt eines Werkes kann mitunter daher rühren, dass die Betrachter unterschiedliche Bezugspunkte für ihre Bewertung der Kreativität zugrunde legen. Ein geschärftes Bewusstsein dafür kann Missverständnissen vorbeugen.

\section{Relevanz der Kreativität im Recht}

Wenngleich auch das Recht bei der Suche nach einer Kreativitätsdefinition nicht hilfreich sein mag, so lässt sich anhand einiger rechtswissenschaftlicher Erkenntnisse bzw. Vorgehensweisen doch die Anwendung der zuvor eingeführten Kreativitätskategorien demonstrieren.

Dabei soll beispielhaft eine Suche nach etwaigen Berührungspunkten von Kreativität zu verschiedenen Rechtsgebieten erfolgen. Das Urheberrecht selbst wird, als Teil des Untersuchungsgegenstandes der Arbeit, in einem späteren Kapitel ${ }^{594}$ ausführlicher unter diesen Gesichtspunkten untersucht.

Zunächst ist festzustellen, dass ein Fehlen von Kreativität bei Rechtssubjekten in Bezug auf ihre Geschäfts- und Schuldfähigkeit zumindest nach dem Text des Gesetzes im Gegensatz zu einem Fehlen von Intelligenz keine Folgen nach sich zieht.

Im Patentrecht wird Kreativität hingegen geschützt und insbesondere gefordert: Im Rahmen des Neuheitserfordernisses in $\S 1$ Abs. 1, 3 Abs. 1 PatG wird absolute Neuheit vorausgesetzt, also $H$-Kreativität gefordert, $P$ Kreativität (in Bezug auf den Erfinder) ist nicht ausreichend. Will man die für den Patentschutz erforderliche Kreativität auch den Typen nach Sloman zuordnen, so dürfte es sich insbesondere um intrinsische Kreativität handeln: Für den Patentschutz kommt es nämlich gerade nicht auf ästhetische, expressive Aspekte an, sondern die Kreativität liegt im Ergebnis

594 S. unten $\S 12$ C.II.. 
mathematischer, physischer und anderer kognitiver Fähigkeiten. Es kann sich hierbei allerdings sowohl um kombinatorische, explorative als auch um transformierende Kreativität handeln. Auch dies ist in Abbildung 12.3 dargestellt.

Jenseits der Kreativität im Recht könnte Kreativität auch am Recht zu suchen sein, so dürfte sich etwa der Rechtsanwender nicht selten im Feld der ersten Sloman-Kategorie (intrinsische Kreativität) betätigen. Neben den allgemein für den Gebrauch und die Erschließung der Rechtssprache erforderlichen kombinatorisch-kreativen Fähigkeiten könnten insbesondere die Analogiebildung und die Anwendung von Auslegungsmethoden Kreativität erfordern.

\section{Analogien}

Der Gesetzgeber ist zwar bemüht, die zu regelnden Situationen möglichst umfangreich zu erfassen, dennoch gibt es immer wieder Sachverhalte, die von einer Vorschrift nicht erfasst zu sein scheinen und dennoch regelungsbedürftig sind. Es liegt dann nahe, aus bekannten, vergleichbaren, geregelten Konstellationen Rückschlüsse auf die „,neue“ Situation zu ziehen. Zu unterscheiden ist zwischen Rechtsanalogien und Gesetzesanalogien. ${ }^{595}$ Erstere bezieht sich auf Analogien, die grundlegende Rechtsgedanken auf konkrete fremde Sachverhalte anwenden Im Gegensatz dazu bedeutet die Gesetzesanalogie eine Anwendung von Rechtsvorschriften auf von diesen eigentlich nicht erfasste abstrakte Fälle, dabei wird „,durch Erweiterung eines Rechtssatzes oder gesetzlichen Tatbestandes ein neuer Rechtssatz geschaffen“. ${ }^{596}$

Der Anfänger lernt als Voraussetzung für die Möglichkeit einer analogen Anwendung einer gesetzlichen Regelung: Erforderlich ist zunächst eine (dem Gesetzgeber unbewusst gebliebene, also nicht gezielt ungeregelte) Regelungslücke und sodann eine Rechtsähnlichkeit und gleichartige Interessenlage des untersuchten Sachverhalts im Vergleich zu geregelten Sachverhalten. ${ }^{597}$ Es gilt also zunächst, überhaupt zu erkennen, dass eine solche Regelungslücke besteht. Zudem muss der Prüfende in der Lage sein, eine Parallele zu bekannten Sachverhalten zu sehen, um dann daraus die korrekten Schlüsse zu ziehen.

595 Weber-Groh, Creifelds, Rechtswörterbuch, Analogie.

596 Weber-Groh, Creifelds, Rechtswörterbuch, Analogie.

597 Mann, Einführung in die juristische Arbeitstechnik, Rn. 274. 


\begin{tabular}{|c|c|c|c|c|}
\hline von & & Kategorien & & Bezugspunkt \\
\hline Miller & c-Kreativität & & C-Kreativität & absolute Neuheit \\
\hline Boden & P-Kreativität & & $\mathrm{H}$-Kreativität & subjektive Neuheit \\
\hline Boden & $\begin{array}{l}\text { kombinatorische } \\
\text { Kreativität }\end{array}$ & $y_{\text {explorative Kreativität }}$ & $\begin{array}{l}\text { transformierende } \\
\text { Kreativität }\end{array}$ & Prozess \\
\hline Sloman & $\begin{array}{l}\text { intrinsische Kreativität } \\
\text { "natürliche Kreativität" }\end{array}$ & & $\begin{array}{l}\text { expressive Kreativität } \\
\text { „Talentkreativität" }\end{array}$ & Ergebnis / Sichtbarkeit \\
\hline
\end{tabular}

Abbildung 12.4: Kreativitätsprofile für Analogie und Auslegung, Quelle: eigene Darstellung.

Auf die Idee der Kreativitätsprofile übertragen ergibt sich das in Abbildung 12.4 dargestellte Bild.

\section{Auslegungsmethodik}

Im Gegensatz zum Analogieschluss ist die Auslegung bestrebt, eine Lesart des Gesetzestextes zu finden, die den vorliegenden Sachverhalt erfasst, obgleich dies nicht auf den ersten Blick ersichtlich ist. Hierfür sind in der juristischen Methodik vor allem die folgenden vier auf Savigny zurückgehenden Auslegungsmethoden anerkannt: ${ }^{598}$

\section{- Grammatische Auslegung _ - Historische Auslegung \\ - Systematische Auslegung _ _ Teleologische Auslegung}

Im Rahmen dieser Herangehensweisen wird die zu untersuchende Norm nicht nur auf ihren Wortlaut und ihren Gesetzeskontext analysiert, sondern auch die Entstehungsgeschichte der Norm berücksichtigt sowie - und hier wird wohl besonders viel intrinsische Kreativität erforderlich sein - im Rahmen der teleologischen Auslegung der Sinn und Zweck, den der Gesetzgeber mit dem Erlass der Norm verfolgte, erforscht, und für die Anwendung zugrunde gelegt. Hier bewegt sich der Rechtsanwender auf dem Feld der Boden'schen explorativen Kreativität. Auch das Kreativitätsprofil der Auslegung ist in Abbildung 12.4 abgebildet.

598 Mann, Einführung in die juristische Arbeitstechnik, Rn. 230 ff., dort finden sich auch Beispiele zu allen Auslegungsarten. 


\section{Folgerung}

Insbesondere die rechtlichen Beispiele zur Kreativität lassen die enge Beziehung derselben zur Intelligenz vermuten - es könnte anzunehmen sein, dass der Rechtsanwender eben ein gewisses Maß an Intelligenz aufweisen muss, um einen Sachverhalt korrekt unter eine gesetzliche Bestimmung zu subsumieren, ohne dass dabei schon gleich seine Kreativität gefordert wäre. Ein zweiter Blick offenbart dann aber, dass gerade die Subsumtion in vielen Fällen ohne ein Element der Auslegung nicht auskommen wird. Dies dürfte zumindest die Sloman'sche intrinsische Kreativität erfordern, womit die Grenzen zwischen Kreativität und Intelligenz mehr und mehr verschwimmen.

\section{Verhältnis von Kreativität und Intelligenz und deren Bedeutung für das Urheberrecht}

Um trotz der unscharfen Grenzen zwischen Intelligenz und Kreativität mit den Konzepten arbeiten zu können, scheint es dienlich, das Verhältnis der beiden Begriffe zu beleuchten. Nach einigen grundsätzlichen Überlegungen erfolgt im Rahmen der Betrachtung des Urheberrechts eine Analyse des urheberrechtlichen Schöpfungsprozesses. Dabei werden zum einen menschliche mit maschinellen „Schöpfungsvorgängen“ kontrastiert und zum anderen stets die Berührungspunkte zu Intelligenz und Kreativität gesucht. Das Ziel der Betrachtung ist es, Merkmale des Schöpfungsprozesses herauszustellen, die naturgemäß eine hohe Abhängigkeit zum Vorliegen eines menschlichen, intelligenten und kreativen Schöpfers aufweisen und die es daher später im Rahmen der „Computational Creativity“ zu berücksichtigen gilt.

\section{Grundsätzliche Überlegungen}

Zunächst ist aufzuzeigen, inwiefern die Unschärfe der Begriffe Herausforderungen für die weitere Betrachtung aufwirft. Im Rahmen einer Gegenüberstellung werden Wechselwirkungen von Intelligenz und Kreativität aufgezeigt. 


\section{Beschreibbarkeitsproblem}

Möglicherweise lässt sich Kreativität als Bestandteil oder „Art“ von Intelligenz auffassen, was wiederum davon abhängt, wie Intelligenz verstanden wird. Festhalten lässt sich nach den oben zusammengetragenen Erkenntnissen, dass eine endgültige Antwort auf die Frage des Verhältnisses von Kreativität und Intelligenz zu einander ebenso aussteht ${ }^{599}$ wie allgemein akzeptierte Definitionen der Begriffe. Letzteres dürfte einen nicht unerheblichen Einfluss auf Ersteres ausüben.

Auch die eingangs ( $§ 12$ B.) aufgestellte These, dass das tatsächliche Vorliegen von Kreativität den Nachweis von Intelligenz erleichtern könnte, ließ sich durch die Untersuchungen in diesem Kapitel nicht erhärten. Was gelingen mag, ist - zumindest für die Kreativität - eine Art Clustering wesentlicher, wenn auch nicht untereinander verbundener Kriterien, wie es in den Abbildungen der Kreativitätsprofile versucht wurde. Von eindeutigen Definitionen, wie sie etwa auch für die vorliegende Untersuchung verwendet werden könnten, scheint man noch weit entfernt zu sein.

\section{Konsequenzen}

Das Fehlen eindeutiger und griffiger Definitionen für Intelligenz und Kreativität ist von einiger Bedeutung für unseren Untersuchungsgegenstand: Bei der landläufigen Verkürzung der Vorstellung künstlicher Kreativität auf Assoziationen insbesondere mit „,künstlerischen“ Systemen, also mit der Erzeugung von Bild- und Tonwerken oder Texten, gerät in den Hintergrund, dass Kunst oft auch versucht, Aussagen zu transportieren, und sich nicht mit der Darstellung „schöner“ Motive begnügt. Das erfordert eine Auseinandersetzung mit einer Bedeutungsebene - und damit auch eine Kombination von Wissen, Erfahrungen und Ansichten mit Farbe, Motiven, Materialien und anderen künstlerischen Ausdrucksmitteln. Diese Kommunikationsfunktion der Kunst lässt sich eher in der intrinsischen Kreativität verorten als in der Talentkrea-

599 Sternberg/O'Hara, Creativity and Intelligence, S. 269, untersuchen unterschiedliche Möglichkeiten des Verhältnisses von Kreativität und Intelligenz anhand verschiedener in der Literatur vorgestellter Theorien, kommen aber dennoch zu dem Schluss, dass (zumindest) „Psychologen noch keine Übereinkunft über die Natur der Beziehung zwischen Kreativität und Intelligenz erzielt haben“; im Ergebnis ebenso 14 Jahre später Jauk et al., Intelligence 41 Nr. 4 2013, S. 212. 
tivität, welche eigentlich am Werk zu vermuten ist, und setzt auch Elemente der analytischen und konstruktiven Intelligenzkomponenten voraus.

Maschinen die Fähigkeit zu verleihen, Bedeutung in ihren Erzeugnissen zu kommunizieren, ist nicht trivial (nicht zuletzt könnte unterstellt werden, eine Maschine ohne Bewusstsein könne nicht in der Lage sein, überhaupt Bedeutung in irgendetwas zu sehen, und diese daher auch nicht in Kunst transportieren). Gleichzeitig bündeln sich in der intrinsischen Kreativität Fähigkeiten, die (auch für „,nicht-künstlerisch begabte“) Menschen verhältnismäßig einfach (und intuitiv) erscheinen, wohingegen die gleichen Aufgaben für Entwickler von KI-Systemen erhebliche Herausforderungen darstellen. ${ }^{600}$ Die Regelung in der DSM-RL, die im wesentlichen den Einsatz sogenannter „Uploadfilter" erforderlich macht, ${ }^{601}$ etwa bedarf eigentlich Systemen, die in der Lage sind, unter anderem Satire zu erkennen, um damit ein Overblocking zu verhindern. Dazu müsste ein eingesetzter Algorithmus in der Lage sein, ein Bild oder Video nicht lediglich als Ansammlung von Pixeln zu erkennen, sondern auch den dadurch transportierten Inhalt verstehen und bewerten können. Denkbar ist, dass die Entwicklung von Systemen, die diese Unterscheidungen zwischen schlichten urheberrechtswidrigen Vervielfältigungen und von Schrankenregelungen gedeckten Nutzungen erkennen können, auch Systeme hervorbringen könnte, die „satirische Kunst“ erzeugen.

\section{Versuch einer Gegenüberstellung}

Auch wenn das Verhältnis zwischen Kreativität und Intelligenz in der Wissenschaft, wie aufgezeigt, nicht abschließend geklärt ist, so lässt sich doch für Zwecke dieser Arbeit versuchen, Elemente einer Beziehung zwischen den Begriffen zu ermitteln. Dies kann zum Beispiel dann interessant werden, wenn man, wie später versucht, für Zwecke der weitere Prüfung die insbesondere vom Urheberrecht unterstellte menschliche Natur des Autors hinwegzudenken unternimmt.

Intelligenz lässt sich - zumindest nach Gottfredson - in eine analytische und eine konstruktive Komponente des Umgangs mit vorgefundener Realität (,unsere Umwelt“) aufgliedern. Wie kreativ sich die Problemlösung darstellt (oder ob sie es nach den in Abbildung 12.2 dargestellten Kreativitätskate-

600 Sloman, The Creative Universe, S. 25.

601 Vgl. Art. 17 DSM-RL. 
gorien überhaupt ist), ist für die Feststellung von Intelligenz offenbar nicht maßgeblich.

Kreativität impliziert nach den im Rahmen der Kreativitätskategorien berücksichtigten Autoren überwiegend die Erschaffung von etwas Neuem. Die Umwelt mag dabei im Sinne einer Aufgaben- oder Problemstellung eine Rolle spielen, diese kann aber durchaus mehr (kombinatorische, intrinsische Kreativität) oder weniger (transformierende, expressive Kreativität) Einfluss auf diesen Schöpfungsakt haben.

Dementsprechend kann für das Verhältnis der beiden Begriffe Folgendes gelten:

- Intelligenz kann sich auch manifestieren, ohne dass dies in einem kreativen Akt geschieht. (Beispiel: die Fähigkeit, die Summe aus 2 und $4 \mathrm{zu}$ errechnen.)

- Kreativität setzt kein bestimmtes (höheres) Maß an Intelligenz voraus. (Beispiel: Ein gemaltes Bild muss keine tiefgründige Botschaft transportieren, sondern kann intuitiv ohne besonderes Wissen entstehen; ausreichend sind die motorische Fähigkeit, einen Pinsel auf einer Leinwand zu führen und die kognitive Erkenntnis, dass aus der Kombination von Farbe, Pinsel und Leinwand ein Bild entstehen kann.)

- Beide Phänomene haben unabhängig voneinander ihre Berechtigung. Intelligenz wird aber eher ohne Kreativität anzutreffen sein, als Kreativität ohne - jedenfalls ein Mindestmaß an - Intelligenz.

- Ein menschliches Wesen setzt keiner der untersuchten Definitionsansätze ausdrücklich voraus.

Die aufgezeigten Wechselwirkungen sollen zumindest dazu beitragen, ein besseres Gefühl für die Abgrenzung und Schnittmengen der Begriffe der „Intelligenz“ und der „Kreativität“ zu entwickeln.

II. Bedeutung von Intelligenz und Kreativität im Urheberrecht

Nachdem nun zumindest ein gewisses Grundverständnis der beiden Konzepte „Intelligenz“ und „Kreativität“ und deren Verhältnis zueinander gefunden werden konnte, erfolgt nun eine Untersuchung dahingehend, wie sich diese Konzepte im Kontext des Urheberrechts auswirken bzw. äußern. 


\begin{tabular}{|c|c|c|c|c|}
\hline von & \multicolumn{3}{|c|}{ Kategorien } & Bezugspunkt \\
\hline Miller & c-Kreativität & & C-Kreativität & absolute Neuheit \\
\hline Boden & P-Kreativität & & H-Kreativität & subjektive Neuheit \\
\hline Boden & $\begin{array}{c}\text { kombinatorische } \\
\text { Kreativität }\end{array}$ & explorative Kreativität & $\begin{array}{c}\text { transformierende } \\
\text { Kreativität }\end{array}$ & Prozess \\
\hline Sloman & $\begin{array}{c}\text { intrinsische Kreativität } \\
\text { „natürliche Kreativität“ }\end{array}$ & & $\begin{array}{c}\text { expressive Kreativität } \\
\text { „Talentkreativität }\end{array}$ & Ergebnis / Sichtbarkeit \\
\hline
\end{tabular}

Abbildung 12.5: Kreativitätsprofil Urheberrecht, Quelle: eigene Darstellung.

\section{Kreativität und Intelligenz als Grundvoraussetzung im Urheberrecht?}

Fraglich ist, ob die Anforderung einer ,persönlichen geistigen Schöpfung“ Mindestanforderungen an den geistigen Zustand des Urhebers stellt. Allerdings wird das Vorliegen dieser Merkmale einer solchen Schöpfung nicht anhand der mentalen Verfassung des Urhebers beurteilt, sondern anhand des infrage stehenden Werkes. Im Urheberrecht wird also kein bestimmter Grad an Intelligenz verlangt, um eine Urheberschaft entstehen zu lassen: Der urheberrechtliche Schaffensprozess ist ein Realakt, weshalb es nicht auf das Alter, die Geschäftsfähigkeit oder die Zurechnungsfähigkeit des Autors ankommt, ${ }^{602}$ wenn es darum geht, eine persönliche geistige Schöpfung festzustellen.

Assoziiert wird das Urheberrecht hingegen oft mit dem Begriff der Kreativität. Dies mag daran liegen, dass das Urheberrecht vorrangig persönliche geistige Schöpfungen zu schützen sucht. Sowohl der Begriff der „Schöpfung“, der sich unter anderem über die englische Übersetzung ,creation “603 mit der Kreativität in Verbindung bringen lässt, als auch die Tatsache, dass es um den Schutz der Leistungen „Kreativer“ (Maler, Dichter, Komponisten, Schriftsteller etc.) geht, tragen wohl dazu bei. Die Kategorisierung der im Urheberrecht mit dem Schutz belohnten Kreativität ist im Rahmen des Kreativitätsprofils in Abbildung 12.5 dargestellt. An dieser Stelle ist besonders offensichtlich, warum in Bezug auf Computerprogramme der Urheberrechtsschutz nicht jedem intuitiv erscheint: Gesucht wird nach einer Form der expressiven Kreativität. Das Ausformulieren von Algorithmen könnte aber auch aufgrund des Schwerpunktes auf Problemlösen und der Nähe zum na-

602 Dreier/Schulze-Schulze, UrhG, § 7 Rn. 3.

603 Obgleich im englischsprachigen Urheberrecht die Kreativität insbesondere im Rahmen der Erfordernis der „Originalität“ („Originality“) relevant ist - und nicht etwa im (sprachlichen) Kontext einer „creation“, vgl. dazu z. B. Gervais, Iowa Law Review 105 Nr. 5 2019, S. 40 ff.. 
turwissenschaftlichen Denken und zur Logik der intrinsischen Kreativität zuzuordnen sein. Es bedarf auch mitnichten besonders schön ausformulierten Computerprogrammcodes, um Urheberrechtsschutz zu erlangen. ${ }^{604}$

2. Von künstlicher Intelligenz über künstliche Kreativität zur hypothetischen Urheberschaft

Möglicherweise kann für Zwecke der urheberrechtlichen Prüfung aber auch auf eine Definition oder Verortung von „Intelligenz“ und „Kreativität“ verzichtet werden: Wenn das, was gesucht wird, nämlich weder (tatsächliche, „echte“) Intelligenz noch (tatsächliche, ,echte“) Kreativität erfordert. Dass Maschinen nicht Menschen gleichzustellen sind, bedarf keiner näheren Erläuterung. Deshalb sei an dieser Stelle der Ansatz verfolgt, das Erfordernis der Menschen-Qualität des Autors - wenn auch nur für dieses Kapitel außer Acht zu lassen. Für die urheberrechtliche Prüfung verbleiben dann die üblichen Prüfungsschritte hinsichtlich der Merkmale einer persönlichen geistigen Schöpfung.

Dies birgt freilich begrifflich weitere Schwierigkeiten: Kann eine Maschine eine persönliche Schöpfung hervorbringen? Kann eine Maschine eine geistige Schöpfung hervorbringen? Und: kann eine Maschine eine Schöpfung hervorbringen? Ist für all diese Schritte die Feststellung von Intelligenz und / oder Kreativität zwingende Voraussetzung?

Wenn die Komponente Mensch in Bezug auf die Urheberqualität für diesen Abschnitt hinweggedacht wird, könnte dies auch auf die Intelligenz und die Kreativität angewandt werden. Es wurde ja bereits festgestellt, dass die von der Wissenschaft speziell für Kreativität aufgestellten Kategorien diese Eigenschaft jedenfalls nicht ausdrücklich voraussetzen. Mithin wäre dann zumindest „,künstliche Kreativität“ zu fordern. Kann dies im Ergebnis auch sicherlich nicht zur Feststellung einer Urheberschaft führen, so könnte die Ermittlung eines hypothetischen Urhebers doch sehr stark darauf hindeuten, dass das entstehende Werk nicht mehr dem Entwickler als Urheber zuzurechnen ist. De lege lata bedeutet dies im Extremfall Gemeinfreiheit. Für die Computational Creativity-Forschung könnte die Anerkennung einer solchen hypothetischen Urheberschaft jedoch - symbolisch - das Erreichen eines weiteren Meilensteins bedeuten.

604 Dreier/Schulze-Dreier, UrhG, § 69a Rn. 28. 
Es folgt eine Analyse des Schöpfungsprozesses in Bezug auf Kreativitätskategorien sowie die Umsetzung der Schöpfung durch „kreative Maschinen“.

a) Menschlicher vs. maschineller Schöpfungsprozess

Vom Ergebnis her gedacht muss die Persönlichkeit des Schöpfers auf irgendeine Art in dem Werkstück Ausdruck finden. Häufig geschieht dies, in dem eine Idee in einem für die gewählte Werkform typischen Medium umgesetzt wird (so könnte sich die Idee, ein grünes Haus zu malen, in dem gemalten Bild manifestieren). Die Umsetzung, gedacht als schlichte Ausführung der Idee, ${ }^{605}$ stellt für Maschinen in der Regel keine Herausforderung dar. Entweder sind die dazu erforderlichen Handlungsschritte bereits vorgegeben oder die Maschine kann durch Lernalgorithmen Fähigkeiten hinzugewinnen (denkbar sind zum Beispiel Lernen durch Beobachtung oder durch Ausprobieren). Die Umsetzung kann in einer Analogie zur menschlichen Werkschöpfung der Motorik gleichgestellt werden, die etwa erforderlich ist, um einen Text zu schreiben, ein Lied zu singen oder ein Bild zu malen.

Kritisch ist hingegen die Inkubation der Idee.

b) Entstehung einer Idee

$\mathrm{Zu}$ den Bestandteilen einer Idee, die in ein Werk umgesetzt werden kann, könnten etwa unter anderem folgende Elemente zählen:

- Thema

- Botschaft

- Wahl des Mediums

- Werkzeuge
- Material

- Beschaffenheit des Werks

- Konzept

- Zielgruppe bzw. Publikum

Insbesondere in Bezug auf das gewählte Thema oder Motiv erfolgt für gewöhnlich eine innerliche Auseinandersetzung des Urhebers mit demselben, aus der die Idee entspringen kann und die potenziell einen wesentlichen Einfluss auf den Charakter des Werkes hat: So kann zum Beispiel ein eher satirisches oder kritisches Werk entstehen, oder der Urheber widmet sein Werk in Verehrung einer Person oder Sache, unterstützt einen (politischen?

605 So wohl schon Platon, vgl. Brodbeck, Zur Philosophie der Kreativität, S. 3. 
gesellschaftlichen? wirtschaftlichen?) Zweck, oder will zur Bildung anderer beitragen. ${ }^{606}$

Die Auseinandersetzung des Urhebers mit dem Thema kann wiederum geprägt sein von seinen eigenen Erfahrungen, seinem Wissen, seinen Emotionen, aber auch von seinem Bewusstsein und seiner Selbstwahrnehmung, und nicht zuletzt auch durch (verschiedene Formen von) Intelligenz und Kreativität: Hier ist zu denken an die drei Formen des Kreativitätsprozesses von Boden: ${ }^{607}$ Die Kreativität in der Auseinandersetzung kann in einer kombinatorischen, explorativen oder transformierenden Art erfolgen.

\section{c) Autonomie im Schöpfungsprozess}

Statt für die Ideenfindung der Maschine Intelligenz zu fordern, könnte darauf abzustellen sein, dass bzw. ob die Maschine (schlicht) autonom handelt, die Idee also aus einem Zustand der Selbstbestimmung heraus entsteht. Hier droht jedoch eine begriffliche Sackgasse: In einem engen Verständnis kann Autonomie aufgefasst werden als das Gegenteil des Programmiertseins, womit eine Autonomie von Maschinen grundsätzlich ausscheiden könnte. ${ }^{608}$ Der Begriff der Autonomie hat viele Facetten, für den hiesigen Zweck dienlich ist eher eine Definition autonomer Systeme, die die Fähigkeit besitzen, auf unbekannte und unvorhergesehene Eingaben zu reagieren und entsprechend zu handeln, ohne dass diese Reaktionen und Handlungen zuvor vollständig beschrieben wurden. ${ }^{609}$

Zudem gilt für Machine Learning-Systeme, dass diese zwar auch programmiert werden, es aber gerade in der Natur dieser Modelle liegt, dass sie durch die Anpassung interner Parameter in Reaktion auf eingelesene Daten im Trainingsprozess ihre interne Logik optimieren, ohne dass dazu weitere Nachjustierungen durch den Menschen erfolgen. Ein ML-System reagiert auf ein Inputdatum am Anfang des Trainingsprozesses anders als am Ende des Trainingsprozesses, wenn etwa mit hohen Zuversichtswerten Labels zugewiesen werden können.

Dennoch kann dem Gesagten unter Ablehnung einer Annahme von Autonomie entgegnet werden, dass auch diese (Weiter-)Entwicklung der Systeme

606 Vgl. auch Lauber-Rönsberg, GRUR 2019, 244, 252.

607 Vgl. oben a).

608 Boden, AI and Creativity: Contradiction?, S. 6.

609 Blackwell/Brown/Young, Live Algorithms: Towards Autonomous Computer Improvisers, S. 150. 
durch den Programmierer angelegt wurde. Noch ist nicht klar, wann und ob tatsächlich und alle Bedenken ausräumende Autonomie in Systemen erreicht wird. Die im nächsten Kapitel vorgestellten Systeme The Painting Fool und ANGELINA könnten bei Umsetzung der Visionen ihrer Entwickler jedoch zumindest sehr nah an ein Verständnis ,echter“ Autonomie heranreichen.

\section{Zusammenfassung}

In diesem Kapitel wurden zunächst die Begriffe der Intelligenz und der Kreativität untersucht. Dabei wurde festgestellt, dass sich weder der eine noch der andere Begriff klar definieren und erfassen lassen, es handelt sich vielmehr um abstrakte Konzepte, die zwar intuitiv verstanden, aber nur ansatzweise erklärt werden können. Dennoch konnten für die Intelligenz zumindest zwei Kernbereiche identifiziert werden: eine analytische und eine konstruktive Komponente.

Im Rahmen der Befassung mit dem Kreativitätsbegriff stellte sich heraus, dass in der Forschung zahlreiche Ansätze existieren, Kreativität zwar nicht zu definieren, aber kreatives Verhalten Kategorien zuzuordnen. Diese Kategorienmodelle zielen dabei auf unterschiedliche Betrachtungsgegenstände eines kreativen Vorgangs ab; die einen stellen das Individuum in den Mittelpunkt, die anderen fassen den Entstehungsprozess ins Auge und wieder andere stellen auf die Wahrnehmbarkeit des Ergebnisses ab. Aus diesen Kategorien erfolgte im Rahmen dieses Kapitels die Entwicklung sogenannter „Kreativitätsprofile“, die es ermöglichen, die Kategorien gemeinsam auf einen Betrachtungsgegenstand anzuwenden und ein Gesamtbild entstehen zu lassen.

Das Kapitel befasste sich des weiteren mit Berührungspunkten des Rechts zu den Bereichen der Kreativität und der Intelligenz, dabei lag der Betrachtungsfokus zunächst auf den Gebieten des Zivil- und Strafrechts, und sodann auch auf der Rechtsanwendung im Rahmen von Auslegungsmethoden und der Bildung von Analogien.

Gesondert wurde im Anschluss das Urheberrecht im Kontext von Intelligenz und Kreativität ins Auge gefasst. Dabei erfolgte insbesondere eine Betrachtung des Schöpfungsprozess im Lichte natürlicher und maschineller „Schöpfungsvorgänge“. Anhand der beschriebenen Elemente des Schöpfungsprozesses, die regelmäßig - wenn auch aufgrund der Menschlichkeit des Urhebers in der Regel implizit - in Bezug auf die Urheberschaft geprüft werden, kann nun eine Analyse von Ansätzen der künstlichen Kreativität 
erfolgen. Dabei wird auf die Merkmale der „Persönlichkeit, die im Werk Ausdruck findet“, die „Inkubation einer Idee“ und die Autonomie, in einer jeweils für den Anwendungsbereich der kreativen Systeme modifizierten Fassung, besonderes Augenmerk gelegt. 


\section{$\S 13$ Computational Creativity}

Die zuvor beschriebenen Fragestellungen (mit Ausnahme des konkreten Bezugs zum Recht) werden seit vielen Jahren unter dem Titel der „Computational Creativity" diskutiert. So findet etwa seit 2010 jährlich die International Conference on Computational Creativity ${ }^{610}$ statt. Ein Blick auf diese Diskussion und Beispiele aus diesem Gebiet sollen helfen, die Auswirkungen der bisher getroffenen rechtlichen Einordnung auf die Praxis zu ermitteln.

Computational Creativity bezeichnet einen Teilbereich der künstlichen Intelligenz, in dem das Potenzial von Computern studiert und genutzt wird, mehr als featurereiche Werkzeuge zu sein und als eigenständige autonome Schöpfer und Co-Schöpfer zu agieren. ${ }^{611}$ Es könnte also analog zu ,künstlicher Intelligenz“ von „künstlicher Kreativität“ gesprochen werden. Die Begriffswahl, die an Stelle des - wie bereits für KI aufgezeigten - Begriff des „Künstlichen“ von einer etwaigen Nachahmung des „Natürlichen“ Abstand nimmt und sich stattdessen maschinenbezogener (,,computational“) darstellt, verdeutlicht, dass hier nicht menschliche Kreativität simuliert, sondern eine eigene Art maschineller Kreativität Gegenstand der Forschung ist.

Fraglich ist, ob im Rahmen von Computational Creativity-Systemen die Programmierer überhaupt Wert darauf legen würden, als Urheber der Erzeugnisse anerkannt zu werden: Ihr Ziel ist es ja gerade, die Unabhängigkeit von ihrem eigenen Beitrag möglichst groß auszugestalten. Es wäre geradezu widersprüchlich, wenn sie sich trotzdem noch selbst als Urheber ansehen würden.

Auch die Kognitionswissenschaftlerin Boden beschäftigt sich auf gewisse Weise mit dem Thema der Computational Creativity. Im Rahmen ihrer Lovelace-Fragen untersucht sie, inwieweit etwa computerwissenschaftliche Erkenntnisse das Verständnis menschlicher Kreativität voranbringen können. Die Fragen gehen zurück auf eine Äußerung von Lady Ada Lovelace Mitte des 19. Jahrhunderts:

$610 \mathrm{http} / / /$ computationalcreativity.net (Stand: 22.02.2021).

611 Definition übersetzt von Veale/Cardoso/Pérez y Pérez, Systematizing Creativity: A Computational View, S. 2: „Computational Creativity (CC) is an emerging branch of artificial intelligence (AI) that studies and exploits the potential of computers to be more than feature-rich tools, and to act as autonomous creators and co-creators in their own right.". 
„The Analytical Engine has no pretensions whatever to originate anything. It can do [only] whatever we know how to order it to perform. “612

Boden entwickelte daraufhin folgende vier Fragen: ${ }^{613}$

1) Können computerwissenschaftliche Vorstellungen helfen zu verstehen, wie menschliche Kreativität möglich ist?

2) Können Computer (jetzt oder in Zukunft) jemals imstande sein, etwas zu tun, was wenigstens schöpferisch wirkt? ${ }^{614}$

3) Könnte ein Computer jemals den Anschein erwecken, Kreativität zu erkennen?

4) Könnte ein Computer jemals selbst tatsächlich schöpferisch sein? ${ }^{615}$

Gegenstand der Computational Creativity dürfte insbesondere die Beantwortung der vierten Frage sein. Die zweite Frage ist wohl bereits jetzt augenscheinlich mit ,Ja“ zu beantworten, sofern keine menschliche Kreativität gefordert wird, denn zahlreiche Systeme sind bereits in der Lage, Erzeugnisse zu produzieren, die in ihrem Erscheinungsbild nicht erkennen lassen, dass sie von einer Maschine produziert wurden, und für Kunst gehalten werden. ${ }^{616}$

In diesem Abschnitt wird nicht weiter auf theoretische Grundlagen der (maschinellen) Kreativitätsforschung eingegangen, sondern zwei ausgewählte Beispiele vorgestellt, in denen die Entwickler die Umsetzung maschineller Kreativität anstreben. Diese Beispiele unterscheiden sich von den in $\S 10 \mathrm{C}$. dargestellten ML-Modellen dahingehend, dass ein größerer Fokus auf Eigenständigkeit im kreativen Prozess liegt: Es handelt sich um das Projekt The Painting Fool und das oben ${ }^{617}$ vorgestellte Projekt ANGELINA. Ersteres wird im Folgenden kurz erläutert. Zur Beschreibung des ANGELINA-Projekts wird zum einen auf b) verwiesen, zum anderen finden sich einige ergänzende Anmerkungen in $\S 13$ B.. Anschließend werden die beiden Systeme anhand ihrer Kreativitätsprofile verglichen.

612 Lovelace, Scientific Memoirs 1843, Note G; Hervorhebungen übernommen von Boden, Creative Mind, S. 16.

613 Dies., Creative Mind, S. 16 f. bzw. Boden/Savigny, Flügel des Geistes, S. 18.

614 Im englischen Originaltext ,appear to be creative“, Boden, Creative Mind, S. 16.

615 Original: „,[could] computers themselves ever really be creative“, Dies., Creative Mind, S. 17.

616 Vgl. z. B. das Experiment auf der Art Basel, 2016, https://www.technologyreview. com/2017/06/30/150666/machine-creativity-beats-some-modern-art/ (Stand: 22.02.2021).

617 S. b). 


\section{A. The Painting Fool}

Ziel dieses in 2006 gestarteten Projekts ist es, eine Software zu programmieren, die eines Tages als selbständige/r Künstler/in anerkannt wird. ${ }^{618}$ Dabei ist nicht beabsichtigt, besonders hochwertige Kunstwerke zu erzeugen: Im Vordergrund steht das Bestreben, eine kreative Software zu erschaffen. ${ }^{619}$

$\mathrm{Zu}$ den Fähigkeiten, die das Programm beherrschen soll, gehört die Simulation einer Reihe von kognitiven und physischen Verhaltensweisen von Menschen, wie etwa das Führen eines Pinsels, aber auch die kritische Wertschätzung des eigenen Werkes und die Wahl von eingesetztem Material und Stil. ${ }^{620}$ Das System „malt“ Portraits von Personen, die entweder ,live“ vor einer Kamera sitzen (von denen dann also ein Foto aufgenommen wird), oder anhand von digitalen Fotos.

The Painting Fool ist ein lernendes System, das anhand einer bereitgestellten Schnittstelle durch Benutzer trainiert werden kann. ${ }^{621}$ Das System hat darüber hinaus Zugriff auf Google, Flickr, Facebook und Twitter. ${ }^{622}$ The Painting Fool malt Portraits, die von seiner „Stimmung“ geprägt werden. Diese wird davon beeinflusst, was The Painting Fool an dem Tag in der Zeitung The Guardian ,gelesen“ hat. ${ }^{623}$ Zudem wird in dem Portrait die Stimmung, die in dem Gesichtsausdruck der zu portraitierenden Person erkannt wird, durch die Auswahl von Stil, Material und Farben wiedergegeben. ${ }^{624}$

Im Rahmen der technischen Umsetzung von The Painting Fool werden inzwischen auch ML-Modelle für Computer Vision (also insbesondere zur Bilderkennung) vor, während und nach dem Portraitierungsprozess eingesetzt. ${ }^{625}$ Dazu gehört auch ein Modell, das eine Zuordnung von visuellen Eindrücken zu verbalen Assoziationen vornehmen kann. ${ }^{626}$ Der Prozess verläuft in Bezug auf dieses Modell wie folgt:

618 Colton, The Painting Fool, S. 4.

619 Ders., The Painting Fool, S. 4 f.

620 Ders., The Painting Fool, S. 5.

621 Ders., The Painting Fool, S. 8.

622 Ders., The Painting Fool, S. 8.

623 Miller, The Artist in the Machine, S. 123.

624 Ders., The Artist in the Machine, S. 124.

625 Colton et al., The Painting Fool Sees! New Projects with the Automated Painter., S. 1.

626 Bestandteil des Projekts „DARCI“, Dies., The Painting Fool Sees! New Projects with the Automated Painter., S. 2. 
Ein eingelesenes Bild wird hinsichtlich seiner Farbregionen segmentiert, für die dann Profile erstellt werden. ${ }^{627}$ Diese werden mit den in der Datenbank vorhandenen Malstilen abgeglichen und es werden die zehn passendsten Stile ausgewählt. ${ }^{628}$ Daraus erfolgte zunächst eine zufällige Auswahl eines zu verwendenden Stils für die zu malende Farbregion; dieser Prozess wurde jedoch inzwischen noch optimiert dahingehend, dass alle zehn ausgewählten Stile am konkreten Bild ausprobiert werden. Die finale Auswahl erfolgt dann erst im Rahmen der versuchten Umsetzung durch das Programm. ${ }^{629}$

Die Resultate sind auf der Website www. thepainting fool . com in Galerien abrufbar, und wurden außerdem in Ausstellungen präsentiert. ${ }^{630}$ 2018 war The Painting Fool „Artist in Residence“ bei CUBRIC, dem Cardiff University's Brain Research Imaging Centre in Großbritannien. ${ }^{631}$

Der Initiator von The Painting Fool, Simon Colton, will die Unabhängigkeit des Programms vom Menschen in Zukunft noch weiter vorantreiben. Dazu gehört, dass The Painting Fool lernt, die eigenen Kunstschöpfungsprozesse zu verändern und sich eigenständig Input zu suchen, sowie die Möglichkeit, mit dem System zu interagieren und Rückfragen zum Schaffensprozess stellen zu können. ${ }^{632}$

Durch den großen Gestaltungsspielraum und die zahlreichen externen Faktoren, die in der Erzeugung der Portraits Berücksichtigung finden, besteht kaum die Möglichkeit, zu wissen, in welcher „Verfassung“ das Programm sich im Zeitpunkt der Portraitierung befindet. ${ }^{633}$ Folglich wird es sehr schwierig, vorauszuahnen, wie das Ergebnis gestaltet wird. Damit dürfte auch die $\mathrm{Zu}$ rechnung zum Programmierer und zum Benutzer als Urheber weitestgehend ausscheiden. ${ }^{634}$

627 Colton et al., The Painting Fool Sees! New Projects with the Automated Painter., S. 4.

628 Dies., The Painting Fool Sees! New Projects with the Automated Painter., S. 4.

629 Dies., The Painting Fool Sees! New Projects with the Automated Painter., S. 4.

630 Z.B. „You Can’t Know My Mind“, Galerie Oberkampf, Paris 2013, http:// www.thepaintingfool.com/galleries/you_cant_know_my_mind/index.html (Stand: 22.02.2021).

631 Vgl. dazu https://www.cardiff.ac.uk/news/view/1128669-painting-fool-at-cubric (Stand: 22.02.2021); http://www.thepaintingfool.com/cubric/ (Stand: 22.02.2021).

632 Miller, The Artist in the Machine, S. 127.

633 Ders., The Artist in the Machine, S. 126.

634 Zur urheberrechtlichen Betrachtung s. auch unten $§ 13$ D.III.1.. 


\section{B. ANGELINA}

Das Spielerzeugungssystem ANGELINA wurde bereits in b) vorgestellt. Dabei wurde festgestellt, dass das System bzw. die Ergebnisse zumindest aktuell noch stark von menschlichem Eingreifen bestimmt sind. Der Schöpfer von ANGELINA, Michael Cook, möchte das System jedoch noch viel mehr sich selbst überlassen. Dazu soll das System in einen Dauerablaufzustand versetzt werden: ANGELINA soll eigenständig bestimmen, wann ein Spiel fertiggestellt ist, und es dann online zur Verfügung stellen. ${ }^{635} \mathrm{Er}$ sieht es auch als eine Möglichkeit, dass ANGELINA sich eigenständig Feedback von Benutzern in Bezug auf den Spaßfaktor der Spiele einholt und die Spiele dahingehend optimiert, dass der Spaßfaktor - ein wichtiges Qualitätsmerkmal für Spiele - gesteigert wird. ${ }^{636}$ Sollte diese Umsetzung tatsächlich erfolgen ${ }^{637}$ scheint eine Zurechnung des Ergebnisses zum Programmierer als Urheber auch hier zweifelhaft. Sowohl die Vielzahl der externen Quellen von Feedback als auch der Umstand, dass er dessen Inhalte schwerlich in den Einzelheiten wird vorhersehen können, werden es dem Programmierer unmöglich machen, das Ergebnis als Multimediawerk in der konkreten Gestalt vorherzusehen und zu prägen. ${ }^{638}$ Diese Ansicht teilt auch der Entwickler:

,[...] my goal for ANGELINA is for it to create things that I would not have thought of. Sometimes I do add ideas into the system to see if it can make connections between them, so I am having some ideas and seeing if ANGELINA can rediscover them. But even then, my ideas are fairly simple - my goal as a researcher is to have the system surprise me. And if it's surprising me, I feel like it would be wrong for me to claim that idea was mine somehow. “639

Er denkt jedoch nicht, dass dies bedeuten sollte, dass ANGELINA deshalb Urheberrechte zustehen sollten. ,ANGELINA can't make decisions on its own, it has no motivations or awareness of the concept of copyright even. “" ${ }^{4} 40$

635 http://www.gamesbyangelina.org/2017/07/angelina-next/ (Stand: 22.02.2021).

$636 \mathrm{https} / / / \mathrm{www} . r o c k p a p e r s h o t g u n . c o m / 2018 / 07 / 04 /$ angelina-is-an-ai-that-makesgames/\#comments (Stand: 22.02.2021), Kommentar vom 04.07.2018 21:50.

637 Dass der Entwickler diese Umsetzung tatsächlich noch in Angriff nehmen möchte, wurde am 16.12.2020 per Mail durch den Entwickler bestätigt, vgl. Anhang.

638 Zur urheberrechtlichen Betrachtung s. auch unten $\S 13$ D.III.2..

639 Mail-Austausch mit Mike Cook, 16.12.2020, s. Anhang.

640 Mail-Austausch mit Mike Cook, 16.12.2020, s. Anhang. 


\begin{tabular}{|c|c|c|c|c|}
\hline von & \multicolumn{3}{|c|}{ Kategorien } & Bezugspunkt \\
\hline Miller & c-Kreativität & & C-Kreativität & absolute Neuheit \\
\hline Boden & P-Kreativität & $8 \ldots \ldots \ldots$ & H-Kreativität & subjektive Neuheit \\
\hline Boden & $\begin{array}{l}\text { kombinatorische } \\
\text { Kreativität }\end{array}$ & explorative Kreativität & $\begin{array}{c}\text { transformierende } \\
\text { Kreativität }\end{array}$ & Prozess \\
\hline Sloman & $\begin{array}{l}\text { intrinsische Kreativität } \\
\text { „natürliche Kreativität“ }\end{array}$ & & $\begin{array}{l}\text { expressive Kreativität } \\
\text { "Talentkreativität }\end{array}$ & Ergebnis / Sichtbarkeit \\
\hline
\end{tabular}

Abbildung 13.1: Kreativitätsprofile für ANGELINA und The Painting Fool, Quelle: eigene Darstellung.

\section{Vergleich von ANGELINA und The Painting Fool anhand ihrer Kreati- vitätsprofile}

Die beiden vorgestellten Projekte zeichnen sich dadurch aus, dass ihre Initiatoren das unabhängige kreative Agieren des jeweiligen Systems anstreben. Damit gehen sie deutlich weiter als die Urheber der oben (\$10 C.) vorgestellten Projekte, die sich bei näherer Betrachtung eher als Hilfsmittel bzw. Werkzeug denn als eigenständig agierende Systeme herausgestellt haben. Deshalb ist hier die Frage zu stellen: Sind ANGELINA und The Painting Fool soweit fortgeschrittene und autonome Systeme, dass ihnen - wenn sie ein Mensch wären, bzw. wenn das Urheberrecht - hypothetisch - auch auf Maschinenkünstler Anwendung fände - Urheberrechte zugestanden würden?

Zunächst bietet sich ein Vergleich der beiden Systeme anhand ihrer Kreativitätsprofile an. Die Profile sind in Abbildung 13.1 visualisiert. Beide Systeme stellen sich den Aufgaben ihrer Programmierer und finden Lösungen, für beide Systeme kann zumindest von c-Kreativität ausgegangen werden. In der Unterscheidung zwischen P- und H-Kreativität fehlt freilich der Mensch als subjektiver Bezugspunkt, hier wird von der ablaufenden Software auszugehen sein. Die Systeme schaffen für sie neue Lösungen, die - aufgrund der Einzigartigkeit der Systeme - möglicherweise sogar als H-kreativ eingestuft werden könnten. Andererseits kann es auch an P-Kreativität scheitern, wenn ein Ergebnis mehrfach produziert wird, dann dürfte es jedoch nicht erneut auf die Beurteilung nach dem Schema ankommen.

In Bezug auf den Prozess ist nach allem, was über ANGELINA bekannt ist, davon auszugehen, dass hier insbesondere die Form der kombinatorischen Kreativität vorliegt. Ein anderes ergibt sich auch nicht aus der Vision des Initiators, die soeben ( $\$ 13$ B.) beschrieben wurde. The Painting Fool schafft 
„neue Ideen bzw. Werkstücke unter Anwendung bekannter Regeln“ ${ }^{641}$ und soll nach der Vision des Initiators auch dazu in der Lage sein, sich selbst neue Regeln zu geben. Damit könnte die Tendenz für The Painting Fool sogar in Richtung transformierender Kreativität gehen.

Schließlich sind beide Systeme, wenn auch The Painting Fool möglicherweise mehr und offensichtlicher als ANGELINA, aufgrund der Ausrichtung auf die Erzeugung von Werkstücken als expressiv kreativ einzuordnen.

\section{Kritische und kreative urheberrechtliche Überlegungen zu Computa- tional Creativity}

Nachdem die Möglichkeiten in Bezug auf die Umsetzung von Kreativität für Maschinen anhand aktueller Beispiele dargestellt wurden, stellt sich die Frage, welche Auswirkungen dies auf die Anwendung des Urheberrechts hat. Dabei könnte auch hinterfragt werden, ob das Urheberrecht dieser technischen Entwicklung noch gerecht wird. Eingegangen wird zunächst kurz, aber kritisch, auf die Frage, ob die Kriterien in $\S 2$ Abs. 2 UrhG noch zeitgemäß sind. Es folgt eine knappe Überlegung zu einer Ausgestaltung eines „Urheberrechts" für nichtmenschliche Urheber. Daran schließt sich eine Betrachtung des Potenzials einer „hypothetischen Urheberschaft“ an.

\section{Zu den Kriterien in $\S 2$ Abs. 2 UrhG}

„Werke im Sinne dieses Gesetzes sind nur persönliche geistige Schöpfungen“, so § 2 Abs. 2 UrhG. Die daraus folgenden Anforderungen für Werke wurden bereits oben (z. B. in $\S 9$ B.) herausgearbeitet, verlangt werden also menschlich-gestalterisches Handeln, geistiger Gehalt, sowie die Wahrnehmbarkeit und Individualität des Werkes. Bekanntlich führt dies aber nicht dazu, dass jedes Produkt menschlicher Hervorbringung schon gleich Urheberrechtsschutz genießen kann. Auch wenn ein Mensch den für die Entstehung eines „Werkes“ kausalen Akt vornimmt, ist das Werk dadurch noch nicht ohne weiteres durch das Urheberrecht geschützt, zu denken ist hier etwa an Erzeugnisse der Zufallskunst, so diese durch die Art ihrer Entstehung nicht die Anforderungen an eine persönliche geistige Schöpfung erfüllen. Für sie gilt, dass sie zwar ohne die Initiative des Menschen nicht entstanden wären,

641 Vgl. oben a). 
aber dennoch die mentale Verbindung zu ihrem „Urheber“ missen lassen können. ${ }^{642}$

Der Sinn solcher Kriterien für die Erteilung eines Schutzrechts kann sich nur im Zusammenspiel mit dem zu erreichenden Schutzziel ergeben. Die Kriterien müssen insofern dem Erreichen des Schutzziels zuträglich sein. Diese Überlegungen führen damit unausweichlich zurück zu den Theorien der Begründung bzw. Rechtfertigung des Urheberrechts. Dabei stehen sich regelmäßig individualistische und kollektivistische bzw. utilitaristische Ansätze gegenüber, oder werden einander ergänzend zur Begründung des Urheberrechts herangezogen. ${ }^{643}$

Erstere stellen den Urheber und seine Beziehung zu seinem Werk in den Mittelpunkt, wie es auch in $\S 11 \mathrm{~S} .1$ UrhG zum Ausdruck kommt, Anwendung findet dieser Begründungsansatz insbesondere in den Droit d'Auteurbasierten kontinentaleuropäischen Urheberrechtssystemen. ${ }^{644}$

Letztere hingegen vertreten, dass Urheberrechte gewährt werden, um den Kreativen - den Urhebern - einen Grund zu geben, Neues zu schaffen und den Kulturbestand zum Nutzen der Gesamtgesellschaft zu erweitern. ${ }^{645}$

Während eine individualistische Begründung des Urheberrechts es angemessen erscheinen lässt, Entwicklern bzw. Benutzern autonomer kreativer Systeme keinen Urheberrechtsschutz für das Resultat ihrer Bemühungen zu gewähren, könnte mit der utilitaristisch-kollektivistischen Perspektive doch hinterfragt werden, ob der Anreiz, den das Urheberrecht durch die Gewährung von Ausschließlichkeitsrechten bietet, im Sinne eines technischen wie kulturellen Fortschritts nicht doch angemessen sein könnte. Andererseits ist

642 Vgl. z. B. Dreier/Schulze-Schulze, UrhG, § 2 Rn. 8; Lauber-Rönsberg, GRUR 2019, 244, 251 sieht darin ein Indiz, dass es nicht allein darauf ankommt, dass ,,die Leistung von einem Menschen stammt" - allerdings ist die Komponente Mensch auch in den hinzukommenden Kriterien so stark präsent, dass diese zumindest nicht abstrakt losgelöst davon untersucht werden können (es sei denn, die Komponente Mensch wird konsequent und gezielt ausgeklammert, vgl. z. B. §12 C.II.).

643 Vgl. z. B. Schack, Zur Rechtfertigung des Urheberrechts als Ausschließlichkeitsrecht, S. 124; Hansen, Warum Urheberrecht?, S. 83; Lauber-Rönsberg, GRUR 2019, 244, 250.

644 Rehbinder/Peukert-Peukert, Urheberrecht, Rn. 75; Schack, Zur Rechtfertigung des Urheberrechts als Ausschließlichkeitsrecht, S. 124.

645 Rehbinder/Peukert-Peukert, Urheberrecht, Rn. 74; Schack, Zur Rechtfertigung des Urheberrechts als Ausschließlichkeitsrecht, S. 124; Hansen, Warum Urheberrecht?, S. 83. 
zu erwägen, ob nicht andere Anreizmodelle außerhalb des Urheberrechts etwa das Patentrecht ${ }^{646}$ - die erwünschte Wirkung erzielen könnten. ${ }^{647}$

Im britischen Recht etwa existiert bereits eine Regelung, die als Autor eines computergenerierten Werkes denjenigen einsetzt, der die notwendigen Vorkehrungen für die Erstellung des Werkes unternommen hat. ${ }^{648}$

Wenn der Gedanke, die Produktion von Kulturgut voranzutreiben, im Mittelpunkt steht, scheint das Urheberrecht intuitiv die richtige Adresse. Voraussetzung dafür ist freilich, dass Erzeugnisse autonomer Systeme überhaupt als Kulturbeiträge aufgefasst werden. Dies dürfte wiederum stark subjektiv davon abhängen, wer die Diskussion führt: Verfechter der „klassischen“, von Menschen geschaffenen Kulturgüter im Sinne etwa der Malerei, der Bildhauerei, der Dichtung und der Musik, oder technikbegeisterte Innovatoren, die die Möglichkeiten, die autonome kreative Systeme bieten, als Chance auffassen, den kulturellen Horizont zu erweitern.

Ins Feld geführt werden könnte insbesondere die Angst, dass menschliche Urheber von künstlichen - maschinellen - „Urhebern“, die immer bessere und immer mehr Leistungen erbringen, verdrängt werden könnten, wenn ihren Leistungen Urheberrechtsschutz zugesprochen würde, ${ }^{649}$ oder, selbst wenn eine Regelung ähnlich der im britischen Recht getroffen würde, aufgrund der schieren Masse der durch automatisierte Produktion immer neu erzeugbaren Werke der Wert geschmälert und der Markt überschwemmt werden könnte.

De lege lata bieten die Anforderungen aus $\S 2$ Abs. 2 UrhG an das Vorliegen eines urheberrechtlichen Werkes noch eine gute „Schutzbarriere“ vor einer dystopischen Kulturrevolution durch kreative autonome Systeme. Aktuell ist zumindest nicht ersichtlich, dass das Kulturwachstum stagniert, weil für nicht erfasste „Maschinenkunst“ den Investoren kein klassischer Urheberschutz gewährt wird. ${ }^{650}$

Dennoch werden im nächsten Abschnitt Ansätze skizziert, die einen Schutz gleichwohl ermöglichen könnten.

646 Wo jedoch die Anforderung der Technizität regelmäßig eine Hürde darstellen dürfte, zu Überlegungen im Patentrecht vgl. z. B. Hetmank/Lauber-Rönsberg, GRUR 2018, 574, 576.

647 Lauber-Rönsberg, GRUR 2019, 244, 250.

648 S. 9 (3) Copyright, Designs and Patents Act 1988: „In the case of a literary, dramatic, musical or artistic work which is computer-generated, the author shall be taken to be the person by whom the arrangements necessary for the creation of the work are undertaken.".

649 Lauber-Rönsberg, GRUR 2019, 244, 252.

650 Ähnlich auch Schricker/Loewenheim-Loewenheim/Leistner, Urheberrecht, §2 Rn. 42; ebenfalls Specht-Riemenschneider, FS Taeger, 711, 720 f.. 
II. Mögliche Ausgestaltung eines „Urheberrechts“ für nichtmenschliche Urheber

In der Diskussion um die Möglichkeiten, Maschinen oder gar Algorithmen Urheberrechte zuzugestehen, wird häufig ${ }^{651}$ schnell das Gegenargument vorgebracht, eine Maschine könne ihr zugestandene Rechte schon gar nicht durchsetzen. ${ }^{652}$ Auch werde dadurch kein Anreiz zur Schaffung weiterer Werke gesetzt, weil eine Maschine eben nicht bedürfnisgesteuert handele. ${ }^{653}$

Dem ist entgegenzuhalten, dass auch im geltenden Recht Urheber mit eigenen Rechten ausgestattet werden, die sie nicht selbst durchsetzen können und deren Empfänglichkeit für eine Anreizwirkung wenn überhaupt beschränkt ist: Die Rede ist von Urhebern mit gesetzlichen Vertretern, z. B. aufgrund von Minderjährigkeit oder anderweitiger Unfähigkeit, die eigenen Ansprüche durchzusetzen.

\section{Durchsetzung durch gesetzlichen Vertreter}

Denkbar wäre, eine Maschine bzw. ein autonom werkeproduzierendes System für rechtsfähig zu erklären: Unter ständigem Beistand durch einen gesetzlichen Vertreter. Dies könnte der Eigentümer des Systems sein. ${ }^{64}$ Die wesentlichen, erforderlichen Handlungen dürften Reaktionen auf Verletzungshandlungen anderer ausmachen. Für diesen Fall könnte der gesetzliche Vertreter die Rechte des Systems geltend machen. Die daraus entstehenden Ansprüche könnten sodann anteilig dem gesetzlichen Vertreter zustehen (als Ersatz seiner Aufwendungen sowie als Anreiz zur Durchsetzung) und anteilig etwa der Kulturförderung zufließen.

$\mathrm{Zu}$ erwägen wäre darüber hinaus die Erteilung einer umfassenden gesetzlich statuierten Erlaubnis zur Verwertung des Werkes. Probleme ergeben sich insbesondere jedoch im Hinblick auf die Beweisbarkeit, so könnte der gesetzliche Vertreter etwa behaupten, das Werk selbst geschaffen zu haben. ${ }^{65}$ Fraglich ist auch, ob und welche Rolle das Urheberpersönlichkeitsrecht noch spielen würde. Zudem wäre dann auch zu bedenken, dass - bzw. ob - der oder die Vertreterin dann auch für - wenn auch schwierig vorstellbare - Hand-

651 So auch in dieser Arbeit oben $\S 9$ A..

652 Vgl. z. B. Legner, ZUM 2019, 807, 809 f.; Bleckat, InTeR 2 2019, 54, 56.

653 Vgl. z. B. Lauber-Rönsberg, GRUR 2019, 244, 251 f..

654 Für Rechte des Verfügungsbefugten vgl. auch Dornis, GRUR 2019, 1252, 1262 f..

655 Ähnliche Probleme sieht auch Lauber-Rönsberg, GRUR 2019, 244, 253. 
lungen des Systems verantwortlich zu machen wäre. ${ }^{656}$ Denkbar wären hier Urheberrechts- oder Persönlichkeitsrechtsverletzungen (wobei es hier freilich nicht auf einen Vorsatz des Systems ankommen kann, was weitere Probleme eröffnen könnte). Das Haftungsrecht im Kontext von autonomen Systemen ist jedoch ein noch viel weiteres Feld, als dass eine hiesige Behandlung dem gerecht werden könnte. ${ }^{657}$

\section{Leistungsschutzrecht de lege ferenda}

Nicht zuletzt aufgrund dieser Herausforderungen wird andernorts vorgeschlagen, ein Leistungsschutzrecht zu schaffen, das - ähnlich dem Schutz für Lichtbilder - nicht an eine geistige Schöpfung gekoppelt ist. ${ }^{658}$ Vorgeschlagen wird etwa ein Leistungsschutzrecht, das an menschliche Handlungen im Rahmen der „Beschaffung und Inbetriebnahme“ eines ML-Systems anknüpft. ${ }^{659}$

Gegen die Idee eines Leistungsschutzrechtes für KI-Erzeugnisse wird jedoch eingewandt, dass - zumindest auf EU-Ebene - angestrebt wurde, das Urheberrecht technologieneutral auszugestalten. ${ }^{660}$ Das Schaffen immer neuer Leistungsschutzrechte untergrabe diese Zielsetzung, indem das eigentliche Urheberrecht unangetastet bleibt und lediglich durch verwandte Schutzrechte ergänzt wird. ${ }^{661}$

\section{Schlussfolgerung und Zusammenfassung}

Die Anerkennung einer Maschinenurheberschaft de lege ferenda - mit oder ohne gesetzlichen Vertreter - scheitert nach wie vor an offenen Fragen zur Haftung und Beweisbarkeit. Ob ein Leistungsschutzrecht de lege ferenda wirklich erforderlich ist, setzt eine tiefgehende wirtschaftliche Analyse voraus.

656 Vgl. Gervais, Iowa Law Review 105 Nr. 5 2019, S. 38.

657 Ausführlich diskutiert zum Beispiel in Kleiner, Die elektronische Person.

658 Dies erwägend u. a. Lauber-Rönsberg, GRUR 2019, 244, 254; Grätz, Künstliche Intelligenz im Urheberrecht, S. 171 ff.; Hetmank/Lauber-Rönsberg, GRUR 2018, 574, 580; Dornis, GRUR 2019, 1252, 1258, 1260 f.; BT-Drs. 19/23700, S. 70 (Bericht der Enquete-Kommission KI); WIPO, Revised Issues Paper, S. 8.

659 Specht-Riemenschneider, FS Taeger, 711, $719 \mathrm{f..}$

660 De Rouck, GRUR Int. 2019, 432, 434, verweisend auf Europäisches Parlament, Bericht mit Empfehlungen an die Kommission zu zivilrechtlichen Regelungen im Bereich Robotik (2015/2103(INL)), A8-0005/2017, Ausführung Nr. 18.

661 De Rouck, GRUR Int. 2019, 432, 435. 


\section{$\S 13$ Computational Creativity}

Ohnehin sind die Schutzlücken nach dem bisherigen Stand der Technik nach der hiesigen Einschätzung minimal bis nicht vorhanden, weil die Systeme (heute) noch nicht so autonom agieren, als dass kein menschlicher Urheber gefunden werden könnte. Und selbst wenn die Zurechnung aufgrund einer allzu großen Zufallskomponente scheitern sollte, gelten eben die gleichen Regeln wie bisher für die Zufallskunst, für die auch keine gesonderten Regeln geschaffen wurden.

Die mögliche Ausgestaltung solcher (Leistungs-)Schutzrechte für KIErzeugnisse wird andernorts bereits ausführlich diskutiert ${ }^{662}$ und hier deshalb nicht thematisiert. Stattdessen liegt der Fokus im Folgenden darauf, wie künstliche Kreativität im Rahmen einer hypothetischen Urheberschaft zumindest bedingt gewürdigt werden kann.

\section{Hypothetische Urheberschaft}

Nachdem in $\S 13$ D.I. festgestellt wurde, dass zunächst an den bestehenden Regelungen des Urheberrechts festgehalten werden sollte, können die in $\S 12$ C.II. erfolgten Überlegungen zur Entstehung einer Idee und zur Autonomie im Schöpfungsprozess auf die beiden vorgestellten Systeme künstlicher Kreativität angewandt werden. Dazu werden die Systeme bzw. ihre Erzeugnisse daraufhin untersucht, ob zu einem positiven Subsumtionsergebnis zu kommen ist, wenn die Komponente Mensch in der Prüfung nicht berücksichtigt wird. Das Vorliegen dieser hier sogenannten ,hypothetischen Urheberschaft" ist ein starkes Indiz dafür, dass das Erzeugnis derart autonom und losgelöst von dem Einfluss des Entwicklers bzw. Nutzers entsteht, dass zwar ein grundsätzlich schutzfähiges Werk, nicht aber menschliche Urheberschaft vorliegt.

\section{The Painting Fool}

Insbesondere The Painting Fool sammelt „Erfahrungen“ in Zeitungsartikeln und in den sozialen Medien, und leitet daraus eine Stimmung ab, die sich in den Erzeugnissen niederschlägt. Wenn auch Abstand davon genommen werden mag, hier von „Persönlichkeit“ zu sprechen, so könnte doch

662 Vgl. z. B. Grätz, Künstliche Intelligenz im Urheberrecht, S. 183 ff.; Dornis, GRUR 2019, 1252, $1260 \mathrm{ff}$.. 
an dessen Stelle das für das System einzigartige Profil, dass sich aus diesen Informationen ergibt, die Persönlichkeit für den Zweck der Prüfung ersetzen. Wenn The Painting Fool in Zukunft außerdem noch eigenständig Motive auswählt, könnte davon gesprochen werden, dass sein Profil ,,in dem Werkstück Ausdruck findet"“. Auch die Inkubation einer Idee könnte dementsprechend angenommen werden: The Painting Fool findet durch die Motivwahl sein eigenes Thema, wählt einen Malstil aus, und integriert unter Umständen auch eine Botschaft, wobei das beschriebene Assoziationsmodell zu berücksichtigen sein wird. Auch die Absicht, überhaupt ein Werk zu erzeugen, könnte durch die Wahl des Motivs - so diese nicht zufällig erfolgt - vorliegen.

Das Erfordernis der Autonomie in der hier eingeschränkten Form der nicht vollständig determinierten Reaktion auf unvorhergesehene Ergebnisse dürfte für The Painting Fool spätestens in der anvisierten Version ebenfalls erfüllt sein: Das System lernt kontinuierlich dazu, und soll in der Lage sein, die eigenen Prozesse anzupassen. Wie The Painting Fool auf unbekannte Inputdaten reagiert, hängt also von viel mehr ab als nur von der ursprünglichen Programmierung. In diesem Sinne dürfte Autonomie also anzunehmen sein.

\section{ANGELINA}

ANGELINA fehlt das Konzept der emotionalen Prägung. Auch hier erfolgt zwar eine Orientierung an Zeitungsartikeln, diese werden aber nicht zur Profilbildung eingesetzt sondern als „Inspiration“ für neue Spielideen herangezogen. ${ }^{663}$ Möglicherweise könnte aber dennoch auf ein Profil abgestellt werden, wenn ANGELINA zugestanden wird, dass ihre bisherigen Erzeugnisse eine gewisse Ähnlichkeit aufweisen, sich durch nicht vorhersehbare in Zeitungsartikeln dargestellte Ereignisse der Erfahrungsschatz erweitert und dadurch ein eigener Stil des Systems vorliegt. Dies könnte dem System allerdings auch als Schwäche ausgelegt werden, wenn angenommen wird, dass ANGELINA sich nur in vorgegebenen Templates bewegt. Hier wird vorerst davon ausgegangen, dass dieser Wiedererkennungseffekt dem Profil des Systems zurechenbar ist, das sich in der Folge in dem Spiel als Werkstück niederschlägt. Nach der Vision des Entwicklers, in der ANGELINA ununterbrochen laufen und eigenständig über die Fertigstellung und die Aufnahme von Spielen bzw. Spielideen entscheiden soll, sind auch die Inkubation einer Idee sowie Autonomie anzunehmen.

663 S. dazu auch die Ausführungen oben b). 
Zur Abgrenzung zu GAN-ML-Systemen ${ }^{664}$ und ähnlichen generativen Systemen ist festzustellen, dass diese - zum aktuellen Stand - im Vergleich zu ANGELINA und The Painting Fool sehr ,schmalspurig“ aufgestellt sind, und in der Regel lediglich Bestandteile eines größeren Systems darstellen. Die hier untersuchten Visionen der Entwickler von The Painting Fool und ANGELINA gehen zudem davon aus, dass ihre Systeme ohne menschlichen Eingriff die Erzeugnisproduktion aufnehmen und sich zudem selbst verändern, während andere Systeme in der Regel die Steuerung durch einen Menschen voraussetzen, der das Programm startet und beendet und die Programmierung bzw. die Trainingsdaten anpasst, um Änderungen in den Ergebnissen zu erzielen.

\section{Schlussfolgerung}

Damit liegen für beide Systeme die modifizierten Voraussetzungen einer hypothetischen Urheberschaft vor, unter der Prämisse, dass die Visionen wie geplant umgesetzt werden. Dies hätte zur Folge, dass die entstehenden „Werke“ keinem menschlichen Urheber zugerechnet werden können, weil der Zurechnungszusammenhang durch den starken autonomen Einfluss des Systems auf das Ergebnis unterbrochen ist.

\section{Zusammenfassung}

Nachdem zunächst festgestellt wurde, dass die Kriterien in § 2 Abs. 2 UrhG nach Würdigung der Rechtfertigung des Urheberrechts und der Berücksichtigung der Besonderheiten künstlicher Kreativität noch zeitgemäß sind, wurden einige alternative Schutzmöglichkeiten aufgezeigt. Die Idee eines Maschinenurhebers, der seine Rechte durch einen gesetzlichen Vertreter durchsetzen lässt, wurde schon aus Gründen der Beweisbarkeitsproblematik rasch verworfen. Für die Diskussion eines Leistungsschutzrechts de lege ferenda wurde auf andere Arbeiten verwiesen.

Sodann wurde das Konzept einer hypothetischen Urheberschaft unter Ausklammern der menschlichen Aspekte in der urheberrechtlichen Subsumtion einer persönlichen geistigen Schöpfung eingeführt. Im Rahmen der hypothetischen Urheberschaft werden der KI zwar keine Urheberrechte zugestanden, aber deren Vorliegen könnte zumindest den Ausschluss einer menschlichen

664 Zum Begriff s. oben $§ 2$ B.II.3.. 
Urheberschaft indizieren und bietet somit eine Erweiterung der Kriterien eines eindeutigen Ausschlusses der Urheberschaft.

\section{E. Fazit}

Nach den oben gesammelten Erkenntnissen kommt es also auf die Frage an, ob man so, wie für die menschliche Urheberschaft „klassische“ Kreativität verlangt wird, für die hypothetische Urheberschaft das Vorliegen von „Computational Creativity“ ausreichen lassen kann. Dies ist zu bejahen: wenn für die mögliche Urheberschaft das Menschsein hinzugedacht werden muss, muss das auch für die Kreativität gelten. Und wenn diese Maschinen Menschen wären und die beschriebenen Werke produzierten, würde wohl nicht an ihrer Kreativität gezweifelt.

Anders sieht das bei den bereits erwähnten Werkzeug-Systemen aus: deren Laufrichtung wird durch Entwickler und Benutzer derart vorgegeben, dass sie, selbst wenn sie Menschen wären, nicht als (alleinige) Urheber anzusehen wären (eine mögliche Miturheberschaft wäre dann zu diskutieren).

Rechtlich kann dieser Unterschied sich allenfalls in der Durchbrechung der Zurechnung des Werkes zum Urheber auswirken, mit der Folge, dass die Erzeugnisse der künstlich kreativen Systeme de lege lata gemeinfrei wären (an dieser Stelle wird bewusst der Konjunktiv verwendet, weil die Ausführungen zu einem wesentlichen Teil auf den Visionen der Entwickler beruhen).

Mit der oben gewonnenen Erkenntnis können zugleich zwei der sogenannten Lovelace-Fragen beantwortet werden: Im Rahmen der hypothetischen Urheberschaft ist die zweite Frage, die darauf abzielt, ob Computer kreativ wirken können, wie auch schon für gängige KI-Kunst, mit einem entschiedenen Ja zu beantworten - vorausgesetzt, die Vorstellung von Kreativität wird von der Menschlichkeit getrennt betrachtet - denn dann könnte möglicherweise auch die vierte Frage zumindest technisch mit einem Ja beantwortet werden.

Boden gibt in Bezug auf die Beantwortung der letzten Frage (,Könnte ein Computer jemals selbst tatsächlich schöpferisch sein?“, bzw. im Original ,[could] computers themselves ever really be creative?") zu bedenken, dass sowohl ein klares Nein als auch ein Ja immer mit Moralvorstellungen einhergehen. ${ }^{665}$ Schließlich schwingt mit der Anerkennung ,echter“ Kreativität

665 Boden, Creative Mind, S. 299. 
auch die Anerkennung von Intelligenz mit, und damit wird Computern eine sonst den höheren Lebewesen vorbehaltene Eigenschaft zugestanden, und damit eine denkbar unüberschaubare Menge an möglichen technischen, sozialen und politischen Diskussionen ausgelöst, für die sicher den meisten Menschen, sofern sie keine Science-Fiction-Autoren sind, die Zeit noch nicht reif erscheint: Könnte das zu künstlichen Richtern, Politikern, Philosophen führen? Mit welchen Folgen?

Die Beantwortung ist also nicht nur von technischer Bedeutung, sondern in einem größeren Kontext zu sehen. Ob die Gesellschaft - die Menschheit die Entscheidung, die vierte Frage zu beantworten, eines Tages treffen wird, bleibt abzuwarten. 


\section{$\S 14$ Ergebnis des fünften Teils}

Der fünfte Teil hat sich zunächst von der reinen rechtlichen Analyse abgewandt und mit den zugrundeliegenden Konzepten der Intelligenz und der Kreativität beschäftigt. Dies sind Konzepte, die sich - bisher - nur schwierig bis nicht erfassen lassen. In der Folge existieren zahlreiche unterschiedliche und bisweilen gegenläufige Vorstellungen von der Materie, die die Übertragung der Konzepte auf andere Situationen - wie die der künstlichen Intelligenz - zu einer Herausforderung werden lassen. Daher konnten zwar einige relevante Aspekte skizziert, aber endgültige Antworten nicht gegeben werden.

Besonderes Augenmerk lag aufgrund der offensichtlichen Nähe zum Urheberrecht auf dem Begriffsverständnis von Kreativität. Nach der Vorstellung der Theorien von Boden, Sloman und Miller ${ }^{666}$ wurde dargelegt, dass auch das Recht Berührungspunkte mit den Begriffen und dem Verständnis von Kreativität und Intelligenz aufweist. ${ }^{667}$ Für ein einfacheres und unmissverständlicheres Verständnis von Kreativität wurde die Idee eines Kreativitätsprofils eingeführt, das Kreativität in Bezug auf die Neuheit, den Urheber, den Prozess und das Ergebnis berücksichtigt und die vorgestellten Theorien zusammenführt. ${ }^{668}$ Sodann erfolgte in Bezug auf das Verhältnis von Kreativität und Intelligenz kurz die Feststellung, dass sich auch die Grenzen der beiden Konzepte nicht klar bestimmen lassen.

Im Anschluss daran wurde ein Bogen zurück zum Urheberrecht geschlagen und nach einer kurzen kritischen Hinterfragung der geltenden Kriterien ${ }^{669}$ und alternativer Schutzmöglichkeiten ${ }^{670}$ untersucht, wie mittels der Konstruktion einer hypothetischen Urheberschaft, die die Voraussetzung der Menschlichkeit außer Acht lässt, ein Anforderungsprofil für künstliche Kreativität geschaffen werden kann. ${ }^{671}$ Es erfolgte sodann eine Anwendung des Anforderungsprofils auf Beispiele aus dem anschließend eingeführten Forschungsfeld der Computational Creativity. Dabei stellte sich heraus, dass die zwei präsentierten Systeme - The Painting Fool und ANGELINA - zumin-

666 S. $§ 12$ B.I.3.

667 S. § 12 A.II., § 12 B.II..

668 S. $\$ 12$ B.I.5..

669 S. $§ 13$ D..

670 S. $§ 13$ D.II..

671 S. $§ 12$ C.II.. 
dest nach der Umsetzung der Visionen ihrer Entwickler (die zumindest auf den ersten Blick technisch realisierbar erscheinen) als tatsächlich künstlich kreativ gelten könnten. Die Systeme wurden anhand ihrer Kreativitätsprofile miteinander verglichen und einer urheberrechtlichen Prüfung anhand des Schemas der hypothetischen Urheberschaft unterzogen. ${ }^{672}$

Anschließend erfolgte die Feststellung, dass sich die urheberrechtliche Auswirkung darauf beschränkt, dass für solche Systeme der Zurechnungszusammenhang zwischen Urheber und Werk durchbrochen wird, womit diese Erzeugnisse de lege lata keinen urheberrechtlichen Schutz genießen.

Abschließend wurde auf die zu Beginn des letzten Kapitels eingeführten Lovelace-Fragen eingegangen und gefolgert, dass die ersten drei Fragen, also jene nach der Analysierbarkeit von Kreativität durch Computer und deren Fähigkeit, zumindest den Anschein eigener Kreativität zu erwecken, inzwischen schon mit ,ja“ zu beantworten sind, dass aber die Beantwortung der möglicherweise ,größten“ letzten Frage nach ,echter Kreativität durch Computer" nicht ohne gesamtgesellschaftliche Abwägungen erfolgen kann, für welche die Zeit noch nicht reif ist. Auch würde dieser erweiterte Blick auf die Folgen einer etwaigen positiven Antwort den Rahmen dieser Arbeit sprengen.

672 S. § 13 D.III. 
Sechster Teil

Gesamtergebnis und Ausblick 


\section{$\S 15$ Gesamtergebnis}

Es folgt zur Beantwortung der zu Beginn der Arbeit formulierten Problemstellungen eine Zusammenfassung der Ergebnisse der vier Hauptteile der Arbeit.

\section{A. Urheberrechtliche Relevanz des ML-Prozesses}

Untersucht wurde, ob das Training von Modellen maschinellen Lernens auch auf urheberrechtlich geschützten Daten ohne Weiteres möglich ist. Dazu erfolgte im Anschluss der Darstellung der technischen Vorgänge ${ }^{673}$ die Analyse, inwiefern im Rahmen des Machine Learning-Prozesses urheberrechtlich relevante Handlungen im Sinne von Vervielfältigungen und Bearbeitungen vorgenommen werden. ${ }^{674}$ Nach der Feststellung, dass zumindest im Rahmen des Einlese- und Datenaufbereitungsvorgangs in der Regel Vervielfältigungen und teilweise auch Bearbeitungen entstehen, wurde die Anwendbarkeit von Ausnahmetatbeständen nach aktueller Rechtslage und unter Berücksichtigung der durch die Umsetzung der DSM-Richtlinie zu erwartenden Änderungen untersucht. ${ }^{675}$

Dabei hat sich ergeben, dass die Bestimmungen der Text- und Data MiningSchranken uneingeschränkt auch auf das Training von ML-Modellen anwendbar sind. In der Folge ist das Training mit geschützten Daten bereits jetzt für die nichtkommerzielle Forschung unproblematisch, und für jedermann, sofern die Vervielfältigungen und Bearbeitungen nur flüchtiger Natur im Sinne des § 44a UrhG sind. Mit der Umsetzung der DSM-Richtlinie 2021 ist zu erwarten, dass jedermann sich auf die Schranken wird berufen können.

\section{B. Urheberrechtlicher Schutz von ML-Modellen}

Die Untersuchung des urheberrechtlichen Schutzes von ML-Modellen erfolgte streng praxisbezogen in Bezug auf künstliche neuronale Netze und

673 S. § 3 A..

674 S. $\$ 3$ B..

675 S. § 4, s. auch Abbildung 4.1. 
Random Forest-Modelle. Dabei wurde nicht auf die zugrundeliegenden statistischen bzw. mathematischen Verfahren rekurriert, sondern die tatsächliche Umsetzung in der Softwareentwicklung mithilfe der verbreiteten Frameworks Keras, TensorFlow, Scikit-learn und PyTorch sowie der Programmiersprache $R$ untersucht. Anhand der Analyse der Technologien auf ihre konkreten Vorgehensweisen im Umgang mit den erstellten ML-Modellen konnten Schutzgegenstände identifiziert werden. ${ }^{676}$

Als Schutzgegenstände unterschieden wurden das trainierte Gesamtmodell, trainierte Parameter, Hyperparameter und das untrainierte Modell. Dem Gesamtmodell kommt Schutz sowohl nach § 4 UrhG als Datenbankwerk, als auch Datenbankherstellerschutz gem. $\S \S 87$ a ff. UrhG und - in Bezug auf den dazugehörigen Quellcode - unter Umständen auch Computerprogrammschutz zu. ${ }^{677}$ Das untrainierte Modell hingegen dürfte nur - bei hinreichender Gestaltungshöhe - Computerprogrammschutz genießen. ${ }^{678}$ Wenn auf die trainierten Parameter eines Random Forest-Modells in $R$ abgestellt wird, fallen diese mit dem ,trainierten Modell“ zusammen wodurch ihnen in der Regel urheberrechtlicher Schutz als Datenbankwerk im Sinne des § 4 Abs. 2 UrhG i. V. m. $§ 4$ Abs. 1 UrhG zukommt, zudem kommt bei relevanten Investitionen auch Datenbankherstellerschutz nach $\S \S 87$ a ff. UrhG in Betracht. ${ }^{679}$ Anders ist die Situation für trainierte Parameter der Python-Modelle: Diese bleiben sowohl mangels persönlicher geistiger Schöpfung als auch fehlenden Investitionsgegenstandes ungeschützt.

Die Sammlung der Hyperparameter hat Datenbankcharakter, weshalb sowohl Schutz nach $\S 4$ UrhG als auch nach $\S \S 87$ a ff. UrhG infrage kommt. ${ }^{680}$

Die Ergebnisse unterscheiden sich insbesondere hinsichtlich des Datenbankschutzes wesentlich von den bisher anderweitig publizierten Annahmen, was auch daran liegen mag, dass hier die konkreten Umstände und die Umsetzung in der Softwareentwicklung untersucht wurden. Abbildung $8.1^{681}$ gibt die Ergebnisse der Untersuchung in einer Tabelle wieder.

676 S. zu den Schutzgegenständen $\S 6$.

677 S. § 7 B.IV..

678 S. $\$ 7$ F..

679 S. dazu $§ 7$ D..

680 S. $\S 7$ E..

681 S. $§ 8$. 


\section{Urheberrechtlicher Schutz von Erzeugnissen generativer ML-Modelle}

Nach der Prüfung des Machine Learning-Prozesses und des Schutzes der Modelle schloss sich eine Untersuchung urheberrechtlicher Schutzmöglichkeiten für mittels ML-Modellen generierte Erzeugnisse an. Anhand einer von Grundsätzen der Zufalls- und Computerkunst ausgehenden Betrachtung wurde ein Weg gefunden, solche Maschinenerzeugnisse menschlichen Urhebern zuzurechnen. Dafür wird ein Dreischritt angewendet: Der Mensch muss ein geeignetes ML-System wählen (oder entwickeln), Parameter zur Erzeugung des Ergebnisses seinen Vorstellungen nach einschränken und ein Ergebnis aus mehreren als ein Werk auswählen. ${ }^{682}$

$\mathrm{Zu}$ berücksichtigen ist dafür, dass in der Regel einige menschliche Anstrengungen erforderlich sind, um ein System überhaupt zufriedenstellende Ergebnisse produzieren zu lassen. Und auch in der Feststellung, dass ein selbst angepasstes bzw. eingestelltes System zufriedenstellende Ergebnisse erzeugt, kann eine Verkörperung der Urheberpersönlichkeit und der geistigen Schöpfung des Urhebers liegen. Zur Unterstützung der Urheberermittlung insbesondere wenn mehrere Personen als Urheber in Betracht kommen, wurde ein Flussdiagramm (Abbildung 10.1683) vorgeschlagen und auf Beispielsysteme angewendet. ${ }^{684}$

\section{Computational Creativity}

Der letzte Teil der Arbeit beschäftigte sich - teilweise losgelöst von Rechtsfragen - mit den Themen Intelligenz und Kreativität. Es erfolgte bewusst keine Entwicklung einheitlicher Definitionen. Insbesondere für die Analyse von Kreativität kam ein vorgeschlagenes Kategorienmodell zum Einsatz, das Erzeugnisse, aber auch Systeme anhand eines „Kreativitätsprofils“ vergleichbar macht. ${ }^{685}$

Es wurden die Berührungspunkte von Intelligenz und Kreativität mit dem Recht, insbesondere mit dem Urheberrecht, untersucht ${ }^{686}$ und das For-

682 S. d).

683 S. $\S 10$ B..

684 Für die Anwendungsbeispiele s. § $10 \mathrm{C}$..

685 S. $\S 12$ B.I.5..

686 Intelligenz und Recht: S. § 12 A.II.; Kreativität und Recht: S. § 12 B.II.; Intelligenz, Kreativität und Urheberrecht: S. § 12 C.II.. 
schungsfeld der Computational Creativity ${ }^{687}$ vorgestellt. Beispielhaft präsentierte die Arbeit zwei Systeme aus dem Gebiet: The Painting Fool ${ }^{688}$ und ANGELINA ${ }^{689}$. Sodann erfolgte die kritische Prüfung der allgemeinen urheberrechtlichen Kriterien des $§ 2$ Abs. 2 UrhG $^{690}$ und der Vorschlag eines Modells „hypothetischer Urheberschaft“ im Sinne eines möglichen Ausschlusskriteriums für menschliche Urheberschaft. ${ }^{691}$ Dieses wurde sodann auf die vorgestellten Projekte The Painting Fool und ANGELINA - unter Berücksichtigung der Visionen der Entwickler - angewendet. ${ }^{692}$

Die Ergebnisse des letzten Teils wurden zudem auf ihre Relevanz für die Beantwortung der zu Beginn von $\S 13$ eingeführten Lovelace-Fragen untersucht. ${ }^{693}$ Das Ergebnis ist, dass prinzipiell eine Beantwortung der vierten Frage - nach ,echter“ Kreativität durch Computer - möglich ist. Die enorme Belastung dieses Themas mit moralischen Aspekten und daraus resultierenden Konsequenzen lässt jedoch offen, ob und wie sich die Menschheit dessen eines Tages annehmen wird.

687 S. $§ 13$.

688 S. $§ 13$ A..

689 S. $§ 13$ B..

690 S. $§ 13$ D..

691 S. $\$ 13$ D.III..

692 S. § 13 D.III.1. und $\S 13$ D.III.2..

693 S. § 13 E.. 


\section{$\S 16$ Ausblick}

„Lebendige Cyborgs werden aus dem Schoß des Anthropozäns hervorkommen“, 694 prophezeit der Naturwissenschaftler James Lovelock. Die Frage, wohin die Reise gehen wird, bleibt offen. Den technischen Fortschritt vorauszusehen scheint mit der durch das Moore'sche Gesetz angenommenen Geschwindigkeit der Entwicklung jenseits des Möglichen. Lovelock nimmt an, dass ,irgendein KI-Apparat bald erfunden wird, der das neue Zeitalter schließlich vollends anbrechen lässt“":695 Ein neues Zeitalter hochentwickelter Technologie, insbesondere künstlicher Intelligenz, das er „Novozän“ nennt.

Das erinnert an das Gedankenexperiment um die junge Alice zu Beginn des fünften Teils, deren absterbende Gehirnzellen nach und nach durch künstliche ausgetauscht wurden. Auch wenn der Umgang mit ihr aufgrund eines unveränderten äußerlichen Erscheinungsbildes und unveränderter Verhaltensweisen vermutlich ein menschlicher bleiben wird, so hätte die Möglichkeit dieses hypothetischen Gehirnnachbaus mitunter weitreichende Konsequenzen. Die Herstellung menschlicher Organe ist bereits im Gange, man beachte hierfür allein die Fortschritte im Organ-3D-Druck. ${ }^{696}$ Zusammengesetzt werden schnell Assoziationen an Frankenstein und Co. geweckt.

Wie im Kontext des Urheberrechts bereits angedeutet, könnte auch über eine Rechtezuweisung an autonome Systeme nachgedacht werden. Das Europäische Parlament hat in einem Bericht bereits die Erwägung eines ePersonenstatus eingebracht:

„Das Europäische Parlament [...] fordert die Kommission auf, bei der Durchführung einer Folgenabschätzung ihres künftigen legislativen Rechtsinstruments die Folgen sämtlicher möglicher Lösungen zu untersuchen, zu analysieren und zu bewerten, und zwar: [...] langfristig einen speziellen rechtlichen Status für Roboter zu schaffen, damit zumindest für die ausgeklügeltsten autonomen Roboter ein Status als elektronische Person festgelegt werden könnte, die für den Ausgleich sämtlicher von ihr verursachten Schäden verantwortlich wäre, sowie möglicherweise die Anwendung einer elektronischen Persönlichkeit auf Fälle, in denen Roboter eigenständige Ent-

694 Lovelock, Novozän, S. 106.

695 Ders., Novozän, S. 104.

696 Vgl. z. B. die Suchergebnisse in Google Scholar: https://scholar.google.com/scholar? $\mathrm{hl}=\mathrm{de} \& \mathrm{as} \_s d t=0 \% 2 \mathrm{C} 5 \& \mathrm{q}=3 \mathrm{~d}+$ print + organs $\& b \operatorname{tnG}=($ Stand: 22.02 .2021$)$. 


\section{§ 16 Ausblick}

scheidungen treffen oder anderweitig auf unabhängige Weise mit Dritten interagieren $[\ldots]^{6697}$

Ähnlich der Beantwortung der vierten Lovelace-Frage (,Können Computer wirklich kreativ sein?") wird die Entscheidung über eine solche Rechtezuweisung durch die implizite Anerkennung der Autonomie der Systeme intensive moralische und gesamtgesellschaftliche Diskussionen voraussetzen.

Sowohl Minsky im Jahr 1992 als auch IBM-KI-Forscher heute sehen eine der größten Herausforderungen in der Modellierung von Verständnis im Sinne des Erreichens eines Zustandes des „,common sense“, also des gesunden Menschenverstandes. ${ }^{698}$ So heißt es etwa, ,manche KI-Technologen sind optimistisch, dass wir die Verstands-Herausforderung in den nächsten fünf bis zehn Jahren meistern werden und weisen darauf hin, dass Deep Learning Teil der Lösung sein könnte. “699

Der Ausblick ließe sich jetzt noch in utopische bis dystopische Dimensionen ausweiten, davon wird an dieser Stelle jedoch abgesehen und auf die entsprechende Science Fiction-Literatur verwiesen. ${ }^{700}$ Vielmehr ist darauf hinzuweisen, dass es jetzt die Aufgabe des Rechts ist, die zu erwartenden Änderungen zu begleiten, sich dem Fortschritt nicht zu verschließen und dadurch ein harmonisches Miteinander von Mensch und Technologie zu ermöglichen.

697 Europäisches Parlament, Bericht mit Empfehlungen an die Kommission zu zivilrechtlichen Regelungen im Bereich Robotik (2015/2103(INL)), A8-0005/2017, Nr. $59 \mathrm{f}$.

698 Minsky, Toshiba Review 47 Nr. 7 1992; https://www.ibm.com/watson/advantagereports/future-of-artificial-intelligence/ai-innovation-equation.html (Stand: 22.02.2021).

699 https://www.ibm.com/watson/advantage-reports/future-of-artificial-intelligence/ ai-innovation-equation.html (Stand: 22.02.2021).

700 Einen interessanten Einblick in eine mögliche Entwicklung bis hin zur Überlegenheit von KI bietet zum Beispiel Schätzing, Die Tyrannei des Schmetterlings. 


\section{Literaturverzeichnis}

Abadi, Martin et al., TensorFlow: A system for large-scale machine learning, in: 12th USENIX Symposium on Operating Systems Design and Implementation (OSDI 16), 2016, S. 265-283.

Ahlberg, Hartwig / Götting, Horst-Peter, Beck'scher Online-Kommentar Urheberrecht, 25. Auflage, 2019 (zitiert: Ahlberg/Götting-Bearb.).

Álvarez, Sonja, Wenn Computer Kunst schaffen, Tagesspiegel online vom 09.03.2020 (abrufbar unter: https://www.tagesspiegel.de/kultur/kreative-ki-wenn-computerkunst-schaffen/25625630.html) - Zugriff am 22.02.2021.

Ancona, Marco et al., Gradient-Based Attribution Methods, in: Samek, Wojciech et al. (Hrsg.): Explainable AI: Interpreting, Explaining and Visualizing Deep Learning, Cham, Schweiz 2019.

Antipov, Grigory et al., Apparent Age Estimation From Face Images Combining General and Children-Specialized Deep Learning Models, in: Proceedings of the IEEE Conference on Computer Vision and Pattern Recognition (CVPR) Workshops, June 2016 (zitiert: Antipov et al., Age Estimation).

Apel, Simon / Kaulartz, Markus, Rechtlicher Schutz von Machine Learning-Modellen, in: RDi Nr.1 2020, S. 24-34.

Asimov, Isaac, I, Robot, New York 1950.

Ders., Meine Freunde, die Roboter: Erzählungen, Band 21531, Meisterwerke der Science Fiction, überarb. Neuausg., 2. Auflage, München 2004.

Beierle, Christoph / Kern-Isberner, Gabriele, Methoden Wissensbasierter Systeme: Grundlagen, Algorithmen, Anwendungen, 6. Auflage, Wiesbaden 2019.

Bell, Jason, Machine Learning, 2. Auflage, Indianapolis, Indiana, USA 2020.

Blackwell, Tim / Brown, Oliver / Young, Michael, Live Algorithms: Towards Autonomous Computer Improvisers, in: Computers and Creativity 2012, S. 147-174.

Blöchl, Bernhard, ,KI ist nicht kreativ“, Süddeutsche Zeitung online vom 27.11.2020 (abrufbar unter: https://www.sueddeutsche.de/muenchen/symposium-ki-ist-nichtkreativ-1.5130099) - Zugriff am 22.02.2021.

Bleckat, Alexander, Intelligente Robotoer als Urheber, in: InTeR 2 2019, S. 54-56.

Boden, Margaret A., The Creative Mind: Myths and Mechanisms, 2. Auflage, London 2004.

Dies., Creativity as a Neuroscientific Mystery, in: Vartanian, Oshin / Bristol, Adam S. / Kaufman, Scott Barry. (Hrsg.): The Neuroscience of Creativity, 2013 (abrufbar unter: http://www.ruskin.tv/maggieb/downloads/Creativity_as_a_Neuroscientific_ Mystery.pdf) - Zugriff am 22.02.2021, S. 3-18.

Dies., Artificial Intelligence and Creativity: A Contradiction in Terms? in: Paul, Elliot Samuel / Kaufman, Scott Barry (Hrsg.): The Philosophy of Creativity, 2014 (abrufbar unter: http://www.ruskin.tv/maggieb/downloads/Artificial_Intelligence_ 


\section{Literaturverzeichnis}

and_Creativity__Contradiction_in_Terms__.pdf.pdf) - Zugriff am 22.02.2021, S. 224-244 (zitiert: Boden, AI and Creativity: Contradiction?).

Boden, Margaret A. / Savigny, Rainer von, Die Flügel des Geistes: Kreativität und künstliche Intelligenz, 1. Auflage, München 1992.

Breiman, Leo, Random forests, in: Machine Learning 45 Nr. 1 2001, S. 5-32.

Brodbeck, Karl-Heinz, Zur Philosophie der Kreativität, in: Residenzvorlesungen Würzburg 2012 (abrufbar unter: http://www.khbrodbeck.homepage.t-online.de/philkrea. pdf) - Zugriff am 22.02.2021.

Brown, Tom B. etal., Language Models are Few-Shot Learners, in: 34th Conference on Neural Information Processing Systems (NeurIPS 2020), Vancouver, Kanada 2020 (abrufbar unter: https://proceedings.neurips.cc//paper/2020/file/ 1457c0d6bfcb4967418bfb8ac142f64a-Paper.pdf) - Zugriff am 22.02.2021.

Buitinck, Lars et al., API design for machine learning software: experiences from the scikit-learn project, in: ECML PKDD Workshop: Languages for Data Mining and Machine Learning, 2013, S. 108-122.

Bundesministerium der Justiz und für Verbraucherschutz, Referentenentwurf des Bundesministeriums der Justiz und für Verbraucherschutz. Entwurf eines Gesetzes zur Anpassung des Urheberrechts an die Erfordernisse des digitalen Binnenmarktes, 02.09.2020 (abrufbar unter: https://www.bmjv.de/SharedDocs/Gesetzgebungsverfahren/Dokumente/

RefE_Urheberrecht.pdf?_blob=publicationFile\&v=7) - Zugriff am 22.02.2021 (zitiert: BMJV, RefE 10/2020).

Dass., DiskE: Erstes Gesetz zur Anpassung des Urheberrechts an die Erfordernisse des digitalen Binnenmarktes, 15.01.2020 (abrufbar unter: https: //www.bmjv.de/SharedDocs/Gesetzgebungsverfahren/DE/Gesetz_I_AnpassungUrheberrecht-dig-Binnenmarkt.html?nn=6712350) - Zugriff am 22.02.2021 (zitiert: BMJV, DiskE 01/2020).

Dass., DiskE: Zweites Gesetz zur Anpassung des Urheberrechts an die Erfordernisse des digitalen Binnenmarktes, 24.06.2020 (abrufbar unter: https://www.bmjv.de/SharedDocs/Gesetzgebungsverfahren/DE/ Gesetz_II_Anpassung-Urheberrecht-dig-Binnenmarkt.html;jsessionid= CFC2102684560869B2632AB7727F5A28.1_cid297?nn=6712350) - Zugriff am 22.02.2021 (zitiert: BMJV, DiskE 06/2020).

Bundesregierung, Entwurf eines Gesetzes zur Anpasssung des Urheberrechts an die Erfordernisse des digitalen Binnenmarktes, 03.02.2021 (abrufbar unter: https://www.bmjv.de/SharedDocs/Gesetzgebungsverfahren/Dokumente/ RegE_Gesetz_Anpassung_Urheberrecht_digitaler_Binnenmarkt.pdf?_blob= publicationFile\&v=5) - Zugriff am 22.02.2021 (zitiert: Bundesregierung, RegE 02/2021).

Dies., Strategie Künstliche Intelligenz der Bundesregierung, 2018 (abrufbar unter: https://www.ki-strategie-deutschland.de/home.html?file=files/downloads/ Nationale_KI-Strategie.pdf) - Zugriff am 22.02.2021. 
Carvalho Brito, António E. S. (Hrsg.), 15th European Concurrent Engineering Conference 2008, ECEC '2008 [and] 5th Future Business Technology Conference, FUBUTEC '2008: April 9 - 11, 2008, Porto, Portugal, Ostend 2008.

Chollet, François et al., Keras, https://keras.io 2015.

Colton, Simon, The Painting Fool: Stories from Building an Automated Painter, in: McCormack, Jon / d'Inverno, Mark (Hrsg.): Computers and Creativity, Berlin/Heidelberg 2012, S. 3-38.

Colton, Simon et al., The Painting Fool Sees! New Projects with the Automated Painter. in: ICCC, 2015, S. 189-196.

Cook, Michael, A Vision for Continuous Automated Game Design, in: The AIIDE-17 Workshop on Experimental AI in Games, 2017, S. 54-60.

Ders., / Colton, Simon / Gow, Jeremy, The ANGELINA Videogame Design System Part I, in: IEEE Transactions On Computational Intelligence and AI in Games 9 Nr. 2 2017, S. 192-203.

Dies., The ANGELINA Videogame Design System - Part II, in: IEEE Transactions On Computational Intelligence and AI in Games 9 Nr. 3 2017, S. 254-266.

Craglia, Massimo et al., Artificial intelligence: A European perspective, Band 29425, EUR, Scientific and technical research series, Luxembourg 2018.

Csikszentmihalyi, Mihaly, Society, culture, and person: A systems view of creativity, in: Sternberg, Robert J. (Hrsg.): The Nature of Creativity, New York 1988.

Datenethikkommission der Bundesregierung, Bundesministerium des Innern, für Bau und Heimat, Gutachten der Datenethikkommission der Bundesregierung, Berlin (abrufbar unter: https://www.bmi.bund.de/SharedDocs/downloads/ $\mathrm{DE} /$ publikationen/themen/it-digitalpolitik/gutachten-datenethikkommission. pdf;jsessionid=BC9F8F092B544530C9F802588B17E92E.2_cid364?_blob= publicationFile\&v=6) - Zugriff am 22.02.2021.

De Rouck, Florian, Moral rights \& AI environments: the unique bond between intelligent agents and their creations, in: GRUR Int. 2019, S. 432-436.

Deutscher Bundestag, WD 10: Kultur, Medien und Sport, Künstliche Intelligenz und Machine Learning. Eine urheberrechtliche Betrachtung, 2018 (abrufbar unter: https://www.bundestag.de/resource/blob/592106/ 74cd41f0bd7bc5684f6defaade176515/WD-10-067-18-pdf-data.pdf) - Zugriff am 22.02.2021.

Dornis, Tim W., Der Schutz künstlicher Kreativität im Immaterialgüterrecht, in: GRUR 2019, S. 1252-1264.

Ders., Die „Schöpfung ohne Schöpfer“ - Klarstellungen zur „KI-Autonomie“ im Urheberund Patentrecht, in: GRUR 2021, S. 784-792.

Dreier, Thomas, Creation and Investment: Artistic and Legal Implications of Computergenerated Works, in: Leser, Hans Georg / Isomura, Tamotsu (Hrsg.): Wege zum japanischen Recht: Festschrift für Zentaro Kitagawa zum 60. Geburtstag am 5. April 1992, 1992, S. 869-888. 


\section{Literaturverzeichnis}

Dreier, Thomas / Schulze, Gernot, Urheberrechtsgesetz: Verwertungsgesellschaftenrecht, Kunsturhebergesetz : Kommentar, 6. Auflage, München 2018 (zitiert: Dreier/Schulze-Bearb.).

Dreßing, H. et al., Begutachtung der Geschäfts- und Testierfähigkeit, in: Der Nervenarzt 85 Nr. 11 2014, S. 1441-1450.

Dreyer, Gunda et al., Urheberrecht: Urheberrechtsgesetz, Verwertungsgesellschaftengesetz, Kunsturhebergesetz, 4. Auflage, Heidelberg 2018, Heidelberger Kommentar (zitiert: DKM-Bearb.).

Ehinger, Patrick / Grünberg, Lara, Der Schutz von Erzeugnissen künstlicher Kreativität im Urheberrecht, in: KuR 4 2019, S. 232-237.

Ehinger, Patrick / Stiemerling, Oliver, Die urheberrechtliche Schutzfähigkeit von Künstlicher Intelligenz am Beispiel von Neuronalen Netzen, in: CR 12 2018, S. 761-770.

Epstein, Ziv etal., Who Gets Credit For AI-Generated Art? in: iScience Nr. 232020 (abrufbar unter: https://www.sciencedirect.com/science/article/pii/ S2589004220307070) - Zugriff am 22.02.2021.

Ertel, Wolfgang, Grundkurs Künstliche Intelligenz: Eine praxisorientierte Einführung, 4. Auflage, Wiesbaden 2016, Computational Intelligence.

European Commission High-Level Expert Group on AI, A definition of AI: Main capabilities and scientific disciplines, 2019 (abrufbar unter: https://ec.europa.eu/ newsroom/dae/document.cfm?doc_id=56341) - Zugriff am 22.02.2021 (zitiert: EC HLEG AI).

Europäische Kommission, Evaluation of Directive 96/9/EC on the legal protection of databases, SWD (2018) 147 final, 2018 (abrufbar unter: https://ec.europa.eu/ newsroom/dae/document.cfm?doc_id=51764) - Zugriff am 22.02.2021 (zitiert: Evaluation DB-RL).

Europäisches Parlament, Bericht mit Empfehlungen an die Kommission zu zivilrechtlichen Regelungen im Bereich Robotik (2015/2103(INL)), A8-0005/2017, 2017 (abrufbar unter: https://www.europarl.europa.eu/doceo/document/A-8-2017-0005_ DE.pdf) - Zugriff am 22.02.2021

Faul, Anita C., A Concise Introduction to Machine Learning. Boca Raton, FL, USA 2020.

Fischer, Peter / Hofer, Peter, Lexikon der Informatik, 15. Auflage, Heidelberg 2011.

Fredrikson, Matt / Jha, Somesh / Ristenpart, Thomas, Model Inversion Attacks That Exploit Confidence Information and Basic Countermeasures, in: Proceedings of the 22nd ACM SIGSAC Conference on Computer and Communications Security, New York, NY, USA 2015, CCS '15, S. 1322-1333.

Freialdenhoven, Jan et al., Do AI generated works qualify for copyright? in: IntellectualProperty 2020, S. 28-31 (abrufbar unter: https://www.deutscheranwaltspiegel. de/intellectualproperty/copyright/do-ai-generated-works-qualify-for-copyright/) Zugriff am 22.02.2021.

Gamma, Erich et al., Design Patterns, Indianapolis, IN, USA 1995.

Gervais, Daniel J., The Machine as Author, in: Iowa Law Review 105 Nr. 5 2019, S. $2053-$ 2106. 
Gomille, Christian, Kreative künstliche Intelligenz und das Urheberrecht, in: JZ Nr. 20 2019, S. 696-975.

Goodfellow, Ian J. et al., Deep Learning. Das umfassende Handbuch: Grundlagen, aktuelle Verfahren und Algorithmen, neue Forschungsansätze. 1. Auflage, Massachusetts, USA 2018.

Dies., et al., Generative Adversarial Nets, in: Proceedings of the 27th International Conference on Neural Information Processing Systems - Volume 2, Cambridge, MA, USA 2014, NIPS'14, S. 2672-2680.

Google, Diskussionsentwurf des Bundesministeriums der Justiz und für Verbraucherschutz (BMJV) für ein Erstes Gesetz zur Anpassung des Urheberrechts an die Erfordernisse des digitalen Binnenmarkts, Stellungnahme vom 31. Januar 2020, (abrufbar unter: https://www.bmjv.de/SharedDocs/Gesetzgebungsverfahren/ Stellungnahmen/2020/Downloads/013120_StellungnahmeAnpassungdes_

Urheberrechts_google_DiskE.pdf?_blob=publicationFile\&v=2) - Zugriff am 22.02.2021 (zitiert: Google, Stellungnahme DiskE 01/2020).

Gottfredson, Linda S., Mainstream Science on Intelligence, in: Intelligence 24 Nr. 1 1997, S. 13-23.

Götting, Horst-Peter et al., Urheberrecht: Kommentar, 5. Auflage, München 2017 (zitiert: Schricker/Loewenheim-Bearb.).

Graf, Alexander, Multitalent für Sprache, (abrufbar unter: https://www.spektrum.de/ news/kuenstliche-intelligenz-der-textgenerator-gpt-3-als-sprachtalent/1756796) Zugriff am 22.02.2021.

Grätz, Axel, Künstliche Intelligenz im Urheberrecht - Eine Analyse der Zurechnungskriterien und der Prinzipien der Verwandten Schutzrechte vor dem Hintergrund artifizieller Erzeugnisse, Köln/Wiesbaden 2021.

Grützmacher, Malte, Urheber-, Leistungs- und Sui-generis-Schutz von Datenbanken: Eine Untersuchung des europäischen, deutschen und britischen Rechts: Zugl.: Kiel, Univ., Diss., 1998, Band N.F., 19, Kieler rechtswissenschaftliche Abhandlungen, 1. Auflage, Baden-Baden 1999 (zitiert: Grützmacher, Datenbanken).

Habel, Dominic, Roboterjournalismus, Baden-Baden 2019.

Haberstumpf, Helmut, Der Schutz elektronischer Datenbanken nach dem Urheberrechtsgesetz, in: GRUR 2003, S. 14-31.

Hacker, Philipp, Immaterialgüterrechtlicher Schutz von Trainingsdaten, in: GRUR 2020, S. 1025-1033.

Hansen, Gerd, Warum Urheberrecht? Die Rechtfertigung des Urheberrechts unter besonderer Berücksichtigung des Nutzerschutzes, Baden-Baden 2009.

Harari, Yuval Noah, Homo Deus. Eine Geschichte von Morgen, München 2017.

Hartmann, Frank / Prinz, Matthias, Immaterialgüterrechtlicher Schutz von Systemen Künstlicher Intelligenz, in: WRP 12 2018, S. 1431-1438.

Hauck, Ronny / Cevc, Baltasar, Patentschutz für Systeme Künstlicher Intelligenz? in: ZGE 11 2019, S. 135-169.

Hawking, Stephen, Kurze Antworten auf große Fragen, 14. Auflage, Stuttgart 2018. 


\section{Literaturverzeichnis}

Herberger, Maximilian, „Künstliche Intelligenz“ und Recht, in: NJW 2018, S. 2825-2829.

Hetmank, Sven / Lauber-Rönsberg, Anne, Künstliche Intelligenz - Herausforderungen für das Immaterialgüterrecht, in: GRUR 2018, S. 574-582.

Hinton, Geoffrey E. / Sejnowski, Terrence J., Unsupervised Learning: Foundations of Neural Computation, Massachusetts, USA 1999, A Bradford Book.

Hoeren, Thomas / Sieber, Ulrich / Holznagel, Bernd (Hrsg.), Handbuch Multimedia-Recht, 50. Auflage, München 2020.

Huang, Cheng-Zhi Anna et al., Counterpoint by Convolution, in: Proceedings of the 18th International Society for Music Information Retrieval Conference, ISMIR 2017, 2019.

Huggenberger, Stefan et al., Neuroanatomie des Menschen, Berlin, Heidelberg 2019.

Hurwitz, Judith et al., Augmented Intelligence: The Business Power of Human-Machine Collaboration, Boca Raton, FL, USA 2020.

Iglesias Portela, Maria / Shamuilia, Sheron / Anderberg, Amanda, Intellectual Property and Artificial Intelligence: A Literature Review: EUR 30017 EN, Luxembourg 2019.

Institute of Electrical and Electronics Engineers, IEEE Standard Glossary of Software Engineering Terminology, New York N.Y. 1990 (abrufbar unter: https://ieeexplore. ieee.org/stamp/stamp.jsp?arnumber=159342).

Jauk, Emanuel etal., The relationship between intelligence and creativity: New support for the threshold hypothesis by means of empirical breakpoint detection, in: Intelligence 41 Nr. 4 2013, S. 212-221.

Johnstone, Mitchell, What it Means to „Clean“ Data and „Train“ Machine Learning Algorithms, towardsdatascience.com vom 27.01.2020 (abrufbar unter: https://towardsdatascience.com/what-it-means-to-clean-data-and-trainmachine-learning-algorithms-5ef2cabd2d76) - Zugriff am 22.02.2021.

Käde, Lisa / Maltzan, Stephanie von, Algorithmen, die nicht vergessen - Sicherheitslücken in Machine-Learning-Modellen und deren Bedeutung für den Schutz der Daten und der Urheberrechte, in: InTeR 4 2020, S. 201-208.

Dies., Die Erklärbarkeit von Künstlicher Intelligenz, in: CR 2020, S. 66-72.

Kahou, Samira Ebrahimi et al., Combining Modality Specific Deep Neural Networks for Emotion Recognition in Video, in: Proceedings of the 15th ACM on International Conference on Multimodal Interaction, New York, NY, USA 2013, ICMI '13, S. 543 -550 (zitiert: Kahou et al., Video Emotion Recognition).

Kaplan, Jerry, Artificial intelligence: What everyone needs to know, New York, NY, USA 2016.

Kaulartz, Markus / Braegelmann, Tom, Rechtshandbuch Artificial Intelligence, München 2020.

Kirchner, Hildebert; Böttcher, Eike (Bearb.), Abkürzungsverzeichnis der Rechtssprache, 9. Auflage, Berlin/Boston 2018.

Klass, Eugen, Data Mining und Text Mining: kleine Unterschiede, große Wirkung, in: Wirtschaftsinformatik \& Management 11 Nr. 4 2019, S. 267-269. 
Kleiner, Cornelius, Die elektronische Person, Baden-Baden 2020.

Koncsik, Imre, Quantum Mind, 1. Auflage, Göttingen 2017.

Kremp, Matthias, Roboterjournalismus - Nachrichtenportal MSN online soll von Maschinen gestaltet werden, Spiegel.de vom 30.05.2020 (abrufbar unter: https://www.spiegel.de/netzwelt/web/roboterjournalismus-nachrichtenportalmsn-soll-von-maschinen-bestueckt-werden-a-1906a96b-e5d9-428f-a654f77e450d48ef) - Zugriff am 22.02.2021.

Kummer, Max, Das urheberrechtlich schützbare Werk, Bern 1968, Abhandlungen zum schweizerischen Recht.

Lauber-Rönsberg, Anne, Autonome „Schöpfung“ - Urheberschaft und Schutzfähigkeit, in: GRUR 2019, S. 244-253.

Legner, Sarah, Erzeugnisse Künstlicher Intelligenz im Urheberrecht, in: ZUM 2019, S. 807-812.

Leistner, Matthias, „Last Exit“ withdrawal? Die Zukunft des Europäischen Datenbankschutzes nach der EuGH-Entscheidung in Sachen BHB v. Hill und dem Evaluierungsbericht der Kommission, in: KuR 9 2007, S. 458-465.

Lenzen, Manuela, Natürliche und künstliche Intelligenz: Einführung in die Kognitionswissenschaft, Frankfurt / New York 2002.

Liaw, Andy / Wiener, Matthew, Classification and Regression by randomForest, in: R News 2 Nr. 3 2002, S. 18-22.

Liebmann, Michael / Gertz, S. David; Schünke, Michael / Schünke, Gabriele (Übers. u. Bearb.), Basiswissen Neuroanatomie, 4. Auflage, New York, Stuttgart 2001.

Linke, David, Urheberrechtlicher Schutz von „KI“ als Computerprogramme - Squeezing today's innovations into yesterday's system? in: GRUR Junge Wissenschaft 2019, S. 29-47.

Ders., / Petrlik, David, „Copyright Work and its Definition with Regard to Originality and $\mathrm{AI}^{\text {" }}$ - Conference Report on the Fourth Binational Seminar of TU Dresden and Charles University in Prague, 27 June 2019, in: GRUR Int. 2020, S. 39-45.

Lobo, Sascha, Realitätsschock. Zehn Lehren aus der Gegenwart, Köln 2019.

Loewenheim, Ulrich (Hrsg.), Handbuch Urheberrecht, 3. Auflage, München 2021 (zitiert: Loewenheim-Bearb.).

Ders., / Leistner, Matthias / Ohly, Ansgar, Urheberrecht, 6. Auflage, München 2020 (zitiert: Schricker/Loewenheim-Bearb.).

Looney, Carl G., Neural Networks as Expert Systems, in: Expert Systems With Applications 6 1993, S. 129-136.

Lovelace, Ada, Notes to A Sketch of the Analytical Engine invented by Charles Babbage, by L.F. Menabrea, in: Scientific Memoirs 1843, S. 666-731.

Lovelock, James, Novozän. Das kommende Zeitalter der Hyperintelligenz, München 2020.

Lubart, Todd / Thornhill-Miller, Branden, Creativity: An Overview of the 7C's of Creative Thought, in: Sternberg, Robert J. / Funke, Joachim (Hrsg.): Psychology of Human Thought, Heidelberg 2019. 
Mann, Thomas und Tettinger, Peter J., Einführung in die juristische Arbeitstechnik, 5. Auflage, München 2015.

McCarthy, John et al., A Proposal for the Dartmouth Summer Research Project on Artificial Intelligence, 1955 (abrufbar unter: http://www-formal.stanford.edu/jmc/ history/dartmouth/dartmouth.html) - Zugriff am 22.02.2021.

McCorduck, Pamela, Machines Who Think: A Personal Inquiry into the History and Prospects of Artificial Intelligence, Natick 2004.

McCormack, Jon / d'Inverno, Mark (Hrsg.), Computers and Creativity, Berlin/Heidelberg 2012.

Dies., On the Future of Computers and Creativity, in: AISB14 Symposium on Computational Creativity, 2014 (abrufbar unter: http://users.monash.edu/ jonmc/research/ Papers/AISB14McCormack-dInverno.pdf) - Zugriff am 22.02.2021.

McCulloch, Warren S. / Pitts, Walter, A Logical Calculus of the Ideas Immanent in Nervous Activity, in: The Bulletin of Mathematical Biophysics Band 5 Nr. 4 1943, S. 115-133.

Merton, Robert King, On the shoulders of giants: A Shandean postscript, Chicago and London 1993.

Miller, Arthur, The Artist in the Machine - The World of AI-Powered Creativity, Cambridge, MA, USA 2019.

Ders., Can machines be more creative than humans? TheGuardian.com vom 04.03.2019 (abrufbar unter: https://www.theguardian.com/technology/2019/mar/ 04/can-machines-be-more-creative-than-humans) - Zugriff am 22.02.2021.

Minsky, Marvin, Semantic Information Processing, Cambridge, MA, USA 1968.

Ders., Future of AI Technology, in: Toshiba Review 47 Nr. 71992 (abrufbar unter: https:// web.media.mit.edu/ minsky/papers/CausalDiversity.txt) - Zugriff am 22.02.2021.

Mohanty, Soumendra / Vyas, Ssachin, How to Compete in the Age of Artificial Intelligence: Implementing a Collaborative Human-Machine Strategy for Your Business, New York, NY, USA 2018.

Möhring, Philipp / Nicolini, Käte, Urheberrecht: UrhG, KUG, VerlG, VGG : Kommentar, 4. Auflage, München 2018 (zitiert: Möhring/Nicolini-Bearb.).

Nebel, Jens / Stiemerling, Oliver, Aktuelle Programmiertechniken und ihr Schutz durch § 69a UrhG, in: CR 1 2016, S. 61-69.

Neisser, Ulric et al., Intelligence: knowns and unknowns. in: American Psychologist 51 Nr. 2 1996, S. 77-101.

Nielsen, Michael A., Neural Networks and Deep Learning, 2015 (abrufbar unter: http: //neuralnetworksanddeeplearning.com) - Zugriff am 22.02.2021.

Niu, Weihua / Sternberg, Robert J., The philosophical roots of Western and Eastern conceptions of creativity, in: Journal of Theoretical and Philosophical Psychology 26 Nr. 1-2 2006, S. 18-38.

Nordemann, Wilhelm / Fromm, Friedrich Karl, Urheberrecht: Kommentar zum Urheberrechtsgesetz, zum Verlagsgesetz und zum Urheberrechtswahrnehmungsgesetz, 12. Auflage, Stuttgart 2018 (zitiert: Nordemann/Fromm-Bearb.). 
Ory, Stephan / Sorge, Christoph, Schöpfung durch Künstliche Intelligenz? in: NJW 2019, S. 710-713.

Osinga, Douwe, Deep Learning Cookbook, Sebastopol, CA, USA 2018.

Papastefanou, Stefan, Genetic Breeding Algorithms als Form des „Machine Learning“ im Urheber- und Patentrecht - Rechtliche Herausforderungen beim Schutz von Algorithmen des Genetic Breeding Models, in: CR 4 2019, S. 209-215.

Paszke, Adam et al., Automatic differentiation in PyTorch, in: NIPS-W, 2017.

Patterson, Josh / Gibson, Adam, Deep Learning, Sebastopol, CA, USA 2017.

Perrault, Raymond et al.; AI Index Steering Committee (Hrsg.), The AI Index 2019 Annual Report, Stanford, CA 2019.

Plutarch / Eyth, Eduard (Übers.), Theseus und Valerius Publicola, in: Plutarch IV: Plutarchs ausgewählte Biographien 2. Auflage, Berlin 1907.

Puppe, Frank, XPS-99: Knowledge-Based Systems: Survey and Future Directions: 5th biannual German Conference on Knowledge-Based Systems, Würzburg, Germany, March 1999, proceedings, Band 1570, Lecture notes in computer science. Lecture notes in artificial intelligence, Berlin, London 1999.

Rahmann, Hinrich / Rahmann, Mathilde, Das Gedächtnis: Neurobiologische Grundlagen, Berlin, Heidelberg 2013.

Rehbinder, Manfred / Peukert, Alexander, Urheberrecht und verwandte Schutzrechte, 18. Auflage, München 2018.

Rokach, Lior / Maimon, Oded Z., Data Mining with Decision Trees: Theory and Applications, Singapur 2008, Series in machine perception and artificial intelligence.

Runco, Mark A. / Jaeger, Garret J., The Standard Definition of Creativity, in: Creativity Research Journal 24 Nr. 1 2012, S. 92-96.

Russell, Stuart J. / Norvig, Peter, Künstliche Intelligenz: ein moderner Ansatz, 3. Auflage, München u.a. 2012.

Säcker, Franz Jürgen etal., Münchener Kommentar zum Bürgerlichen Gesetzbuch, 8. Auflage, München 2018 (zitiert: MüKo-Bearb.).

Sammut, Claude / Webb, Geoffrey I. (Hrsg.), Encyclopedia of Machine Learning and Data Mining, New York, NY, USA 2017.

Schack, Haimo, Zur Rechtfertigung des Urheberrechts als Ausschließlichkeitsrecht, in: Geistiges Eigentum: Schutzrecht oder Ausbeutungstitel? Berlin, Heidelberg 2008, S. $123-140$.

Schätzing, Frank, Die Tyrannei des Schmetterlings, Köln 2018.

Schwab, Katharine, 3 reasons why AI will never match human creativity, fastcompany.com vom 25.04.2019 (abrufbar unter: https://www.fastcompany.com/90339590/3reasons-why-ai-will-never-match-human-creativity) - Zugriff am 22.02.2021.

Searle, John, Minds, Brains, and Programs, in: The Behavioral and Brain Sciences 3 1980, S. 417-157.

Sendrowski, Heiko, Zum Schutzrecht „sui generis“ an Datenbanken, in: GRUR 2005, S. 369-377. 


\section{Literaturverzeichnis}

Shannon, Claude E. / McCarthy, John, Automata Studies. (AM-34), Princeton, NJ, USA 1956, Annals of Mathematics Studies 34.

Sloman, Aaron, The Creative Universe (Originaltitel: Creativity and AI), 2019 (abrufbar unter: http://www.cs.bham.ac.uk/research/projects/cogaff/misc/creativity.pdf) Zugriff am 22.02.2021.

Söbbing, Thomas, Fundamentale Rechtsfragen zur künstlichen Intelligenz. (AI Law), 1. Auflage, Frankfurt am Main 2019.

Ders., Algorithmen und urheberrechtlicher Schutz, in: CR 4 2020, S. 223-228.

Ders., Künstliche neuronale Netze, in: MMR 2021, S. 111-116.

Song, Congzheng / Ristenpart, Thomas / Shmatikov, Vitaly, Machine Learning Models that Remember Too Much, in: Proceedings of the 2017 ACM SIGSAC Conference on Computer and Communications Security, 2017.

Specht-Riemenschneider, Louisa, Urheberrechtlicher Schutz für Algorithmenerzeugnisse? - Phasenmodell de lege lata, Investitionsschutz de lege ferenda? in: SpechtRiemenschneider, Louisa/Buchner, Benedikt/Heinze, Christian/Thomsen, Oliver (Hrsg.): IT-Recht in Wissenschaft und Praxis: Festschrift für Jürgen Taeger, Frankfurt am Main 2020 (zitiert: Specht-Riemenschneider, FS Taeger).

Spindler, Gerald, Kurz-Stellungnahme zum DiskE, 04.02.2020 (abrufbar unter: https://www.bmjv.de/SharedDocs/Gesetzgebungsverfahren/Stellungnahmen/ 2020/Downloads/020420_StellungnahmeAnpassungdes_Urheberrechts_GeraldSpindler_DiskE.pdf?_blob=publicationFile\&v=2) - Zugriff am 22.02.2021.

Ders., Text und Data Mining - urheber- und datenschutzrechtliche Fragen, in: GRUR 2016, S. 1112-1120.

Ders., Copyright Law and Artificial Intelligence, in: IIC 2019, S. 1049-1051.

Ders., / Schuster, Fabian, Recht der elektronischen Medien, 4. Auflage, München 2019 (zitiert: Spindler/Schuster-Bearb.).

Stein, Morris I., Creativity and Culture, in: The Journal of Psychology 36 1953, S. 311322.

Stephan, Achim / Walter, Sven, Handbuch Kognitionswissenschaft, Stuttgart 2013.

Sternberg, Robert J., Beyond IQ: A Triarchic Theory of Human Intelligence, New York, NY, USA 1985, Cambridge paperback library (zitiert: Sternberg, Beyond IQ).

Ders., The Nature of Creativity. Contemporary psychological perspectives, New York, NY, USA 1988 (zitiert: The Nature of Creativity).

Ders., Handbook of Creativity, New York, NY, USA 1999.

Ders., / O'Hara, Linda A., Creativity and Intelligence, in: Handbook of Creativity 1999, S. 251-272.

Still, Arthur / d'Inverno, Mark, A History of Creativity for Future AI Research, in: Proceedings of the Seventh International Conference of Computational Creativity, 2016, S. 147-154.

Turing, Alan M., Computing Machinery and Intelligence, in: Mind LIX Nr. 49 1950, S. 433-460. 
Ders., Intelligent Machinery, in: Teuscher, Christof (Hrsg.): Turing's Connectionsim. Discrete Mathematics and Theoretical Computer Science, verfassst 1948, veröffentlicht 2002 (abrufbar unter: https://weightagnostic.github.io/papers/turing 1948. pdf) - Zugriff am 22.02.2021, S. 17-62.

Veale, Tony / Cardoso, F. Amílcar (Hrsg.), Computational Creativity, Cham 2019.

Veale, Tony F. / Cardoso, F. Amílcar / Pérez y Pérez, Rafael, Systematizing Creativity: A Computational View, in: Computational Creativity 2019, S. 2-19.

Vincent, James, How Three French Students Used Borrowed Code to Put the First AI Portrait in Christie's, theverge.com vom 23.10.2018 (abrufbar unter: https://www.theverge.com/2018/10/23/18013190/ai-art-portrait-auctionchristies-belamy-obvious-robbie-barrat-gans) - Zugriff am 22.02.2021.

Vogel, Evelyn, Kreativität und Codes, Süddeutsche Zeitung online vom 16.09.2020 (abrufbar unter: https://www.sueddeutsche.de/muenchen/kuenstliche-intelligenzkreativitaet-und-codes-1.5027491) - Zugriff am 22.02.2021.

von Maltzan, Stephanie / Käde, Lisa, Algorithmen, die nicht vergessen - Model Inversion Attacks und deren Bedeutung für den Schutz der Daten und der Urheberrechte, in: Taeger, Juergen (Hrsg.): Den Wandel begleiten - IT-rechtliche Herausforderungen der Digitalisierung, 2020, S. 505-524.

Wandtke, Artur-Axel / Bullinger, Winfried (Hrsg.), Praxiskommentar zum Urheberrecht, 5. Auflage, München 2019 (zitiert: Wandtke/Bullinger-Bearb.).

Weber, Klaus (Hrsg.), Creifelds, Rechtswörterbuch, 24. Auflage, München 2020.

Wiebe, Andreas, Schutz von Maschinendaten durch das sui-generis-Schutzrecht für Datenbanken, in: GRUR 2017, S. 338-345.

Wikimedia Deutschland, Gesellschaft zur Förderung Freien Wissens e.V., Stellungnahme des Wikimedia Deutschland, Gesellschaft zur Förderung Freien Wissens e.V. zum Diskussionsentwurf des Bundesministeriums der Justiz und für Verbraucherschutz zu einem Ersten Gesetz zur Anpassung des Urheberrechts an die Erfordernisse des digitalen Binnenmarkts, 31.01.2020 (abrufbar unter: https://www.bmjv.de/SharedDocs/Gesetzgebungsverfahren/Stellungnahmen/ 2020/Downloads/013120_StellungnahmeAnpassungdes_Urheberrechts_ wikimedia_DiskE.pdf?_blob=publicationFile\&v=2) - Zugriff am 22.02.2021 (zitiert: Wikimedia Deutschland, Stellungnahme DiskE 01/2020).

World Intellectual Property Organisation, WIPO Secretariat, WIPO Conversation on Intellectual Property (IP) and Artificial Intelligence (AI). Second Session. Revised Issues Paper on Intellectual Property and Artificial Intelligence, 2020 (abrufbar unter: https://www.wipo.int/edocs/mdocs/mdocs/en/wipo_ip_ai_2_ge_20/wipo_ ip_ai_2_ge_20_1_rev.pdf) - Zugriff am 22.02.2021 (zitiert: WIPO Revised Issues Paper).

Zech, Herbert, Künstliche Intelligenz und Haftungsfragen, in: ZfPW 2019, S. 198-219. 


\section{Anhang Mail-Austausch mit Mike Cook, Projekt ANGELINA}

\section{Lisa Käde}

\section{Von:}

Gesendet:

An:

Betreff:

\section{Mike Cook}

Dienstag, 16. Februar 2021 15:01

Lisa Käde

Re: Questions about ANGELINA

Hey Lisa,

Congratulations! Well done on finishing. It's absolutely okay to include our correspondence. Good luck with the end! Mike

On Tue, Feb 16, 2021 at 10:12 AM Lisa Käde

wrote:

Hi Mike,

I hope you are doing well!

I am just about to finish up my thesis and was wondering if you'd be OK with me including our mail exchange in the appendix just in order for me to be able to cite you properly?

Please let me know if this is OK with you.

Thank you very much!

Best regards

Lisa

Von: Mike Cook

Gesendet: Freitag, 18. Dezember 2020 18:16

An: Käde, Lisa (IIWR) <lisa.kaede@kit.edu>

Betreff: Re: Questions about ANGELINA

I am, back in London! I miss Germany very much though. Especially at this time of year, of course. 


\section{Mail-Austausch mit Mike Cook, Projekt ANGELINA}

Thanks again and let me know if you need anything else! Fröhes Fest!

Mike

On Fri, Dec 18, 2020 at 7:16 AM Käde, Lisa (IIWR) <lisa.kaede@kit.edu> wrote:

Hi Mike,

wow, this is exciting! Thank you so much for your replies, I find it fascinating to hear about your visions and your thoughts on copyright. I like to ask this third question (I know that it is rather unusual), because I feel it sometimes indicates how connected a developer feels with a program, whether they see it as a tool for creating their own art or whether they actually want it to do its own thing. After all, copyright is all about the connection of an author and the work.

I started incorporating your answers into my chapter and if it's ok I'll let you know about any more questions as they come up.

Yes, I am based in Karlsruhe! I have never been to Kaiserslautern, unfortunately - you're in the UK now probably?

All the best

-Lisa

Von: Mike Cook

Gesendet: Mittwoch, 16. Dezember 2020 15:35:24

An: Käde, Lisa (IIWR)

Betreff: Re: Questions about ANGELINA

Ah I just noticed you're based in Karlsruhe! I was living in Kaiserslautern until about three months ago. It's a nice part of the country! 
On Wed, Dec 16, 2020 at 2:34 PM Mike Cook

wrote:

Hi Lisa!

Thanks for emailing me. It's really exciting to have interest from scholars in other fields, I learn a lot from talking to you.

$>1$. So for starters I'd like to know if your vision for ANGELINA, as I summed it up, is still what you are going for (mainly looking for a confirmation here, but if you want to / can elaborate on any of these that'd be great)

Yes - all of these are still my plans. Some of them may come sooner or later than others. In particular, running playtests with players will be quite challenging I think, from an engineering perspective as well as an Al perspective. But I think this is the way forward. The main change I've made is I've decided to move forward with making ANGELINA a downloadable program. I want to explore how people can incorporate creative Al agents into their lives, and so I'm planning to rework the system (and give it a new name, after 10 years) and allow people to download it and run it on their own computers. I imagine very few people will do so, and at first it will be very broken, but I think it's useful to force myself to do this now because otherwise these things never happen.

I'll still think of my local copy as the 'main' version though, even if it's just in my head :)

$>2$. Are you or are you planning on using generative machine learning techniques?

Not currently. I haven't seen anything I'd want to use them for right now. They have a lot of drawbacks for me, particularly their lack of transparency (they're hard to communicate to people, and also people don't have a very good understanding of how they work, and a big goal for me is to make sure people understand the Al and feel like they can relate to its process and what it does). They also require a lot of data to train, usually, and need quick and easy ways to evaluate outputs, and both of those things are quite complicated for game design.

That said, it is possible I could use generative ML for smaller subtasks - for example, generating art for the game (even temporary art we replace later). I don't want to rule that out. But that will be more like ANGELINA using ML as a tool, rather than building ML into ANGELINA. 


\section{Mail-Austausch mit Mike Cook, Projekt ANGELINA}

>3. Would you feel that someone is infringing upon your copyrights if they copied a game from ANGELINA? If so, do you think that would change the more ANGELINA does it's "own" thing? Or would you say that whatever ANGELINA is able to achieve some day, it would still all be your doing? (This is leaning a little toward Simon Colton's ambition of creating an artist in it's own right..)

Amazing question. I don't think anyone's ever asked me this before.

I think you specifically mean my copyright, as in, Michael Cook's copyright. To that I would say I would not feel they were infringing on my copyright at all, even at this stage where ANGELINA is still quite a basic system. I have actually joked with people that I am quite excited for the day someone steals an idea from ANGELINA, because it will be a very strong evidence that the system invented something cool. People will often say nice things or praise a game, even in user studies, but often they are positively biased or being nice. But if someone stole a game idea? That would mean they thought it was such a good idea it was worth stealing. That would be very exciting.

But no, I don't consider it to be my copyright. That said, I am quite used to the idea of game cloning - it's already quite hard to stop, and it happens quite often in some platforms (like mobile).

I think this might be influenced partly by my position and background? For example, as a researcher I'm quite used to releasing ideas into the public domain. But really, my goal for ANGELINA is for it to create things that I would not have thought of. Sometimes I do add ideas into the system to see if it can make connections between them, so I am having some ideas and seeing if ANGELINA can rediscover them. But even then, my ideas are fairly simple - my goal as a researcher is to have the system surprise me. And if it's surprising me, I feel like it would be wrong for me to claim that idea was mine somehow.

That's not to say I believe the copyright belongs to ANGELINA. I don't really know what that would mean. ANGELINA can't make decisions on its own, it has no motivations or awareness of the concept of copyright even. It also doesn't mean that I wouldn't be tempted to assert copyright. For example, if ANGELINA invented a really good game, and I could fund my research by commercialising that game, it would be tempting. It would mean I could do this research without worrying about other pressures like finding grant funding. So there's some flexibility in my mind, I guess.

But for the most part, I imagine I would just be excited to see someone steal its ideas. :)

Please let me know if you have any more questions! All the best, 


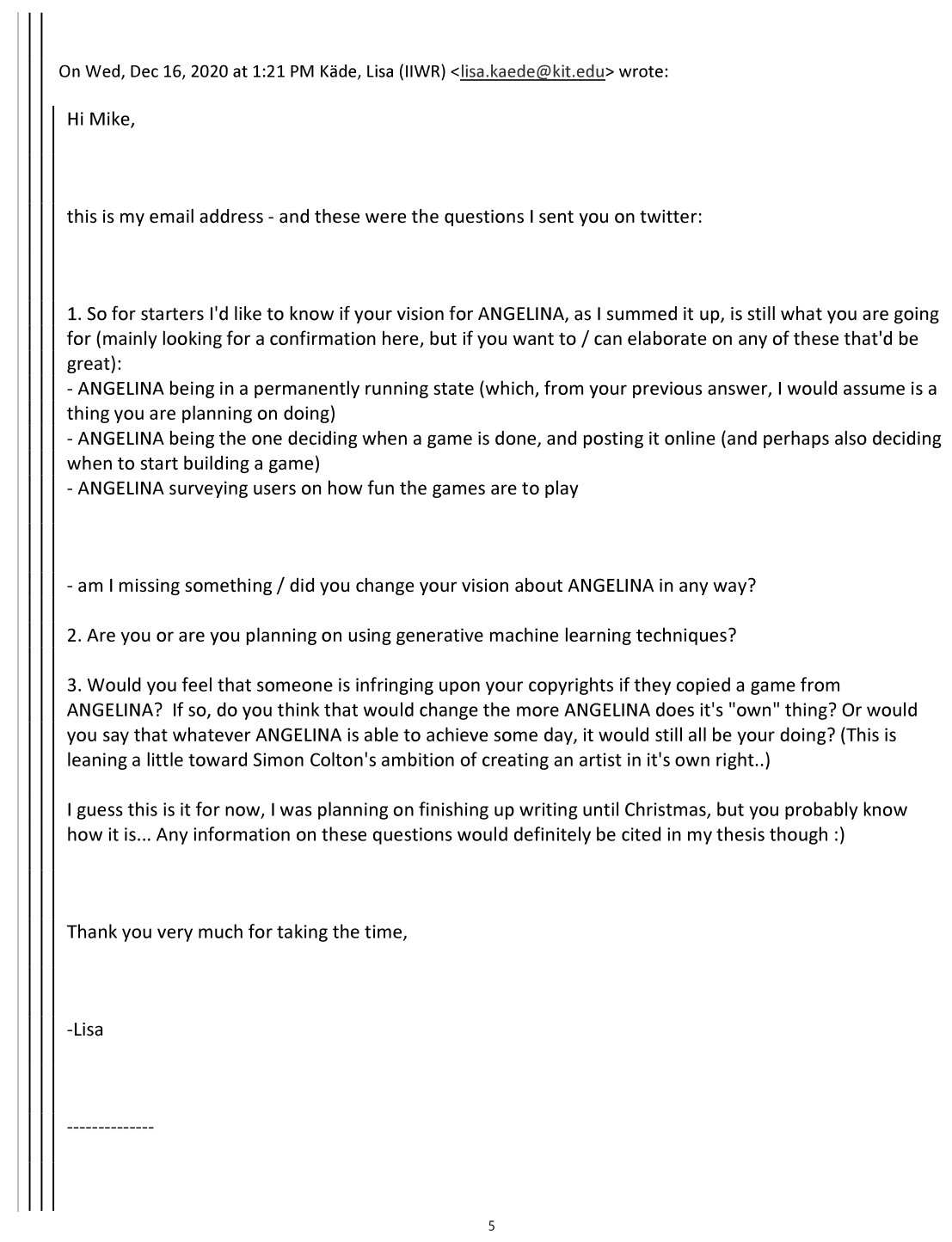

